\title{
HYDROGEN EVOLUTION CATALYZED BY COBALOXIMES
}

\author{
Thesis by \\ Jillian Lee Dempsey \\ In Partial Fulfillment of the Requirements \\ for the Degree of
}

Doctor of Philosophy

California Institute of Technology

Pasadena, California

2011

(Defended November 23, 2010) 
(C) 2011

Jillian Lee Dempsey

All Rights Reserved 
For my mom 


\section{ACKNOWLEDGEMENTS}

I would first like to thank Harry for his guidance over the last five years. Harry has supported my growth as a scientist beyond my wildest expectations-in addition to being the most sincere and caring mentor I could have hoped for, he has given me countless opportunities to define myself beyond the confines of the laboratory and gain exposure in the chemistry community. I could not have made it through these projects without his insight and encouragement, and for that I am truly blessed by this graduate experience.

Second, I would like to thank Jay for his support and advice, teaching me everything I know about kinetics and spectroscopy, building me a diode array spectrometer, and routinely reminding me of the value of fundamental research. Like the best of mentors, he has challenged me to be an assertive researcher and calmly watched me freak out more than once. This thesis would not be nearly as comprehensive without his guidance.

I have had some of the most rewarding scientific discussions of my graduate career with my thesis committee members, Mitchio Okumura, Nate Lewis, and Tom Miller, and I want to thank them for their support over the last five years. I would especially like to acknowledge Nate for giving me the opportunity to cochair the 2009 Renewable Energy: Solar Fuels Gordon-Kenan Graduate Research Seminar. I also want to thank Bruce Brunschwig and Jonas Peters who provided a good deal of guidance on this project.

My undergraduate research advisor, Dan Nocera, has continued to support, encourage, and advise me over the five and a half years since I graduated from MIT. It is from Dan that I gained my love for group theory (5.04) and inorganic spectroscopy, and I am grateful for his continued guidance. 
Participating in the CCI has been a truly rewarding experience, and I am thankful for the many great students, postdocs, and PIs I have interacted with as part of the program, notably Yogi Surendranath, Jennifer Schuttlefield, Craig Markum, Bruce Parkinson, Siddharth Dasgupta, Debbie Hawks, and the Blair High School SHArK team.

I have enjoyed collaborating with a great many researchers during my graduate work. Etsuko Fujita, Dmitry Polyanskiy, and Jim Muckerman at Brookhaven National Laboratory have been enthusiastic about my research for many years and welcomed me into their labs for two visits. Etsuko in particular has been a great friend and role model and I look forward to maintaining our relationship for years to come. It has been a pleasure to make progress towards powering the planet with researchers in both the Lewis and Peters groups. Leslie O'Leary, Judy Lattimer, and Emily Warren have all made contributions towards anchoring cobaloxime catalysts to silicon electrodes, and Xile $\mathrm{Hu}$, Louise Berben, Nate Szymczak, and Charles McCrory have all taught me a great deal about electrochemistry, synthesis, and catalytic hydrogen evolution.

I have had the opportunity to work with a number of talented undergraduates at Caltech. Carolyn Valdez has been a great friend and I have been truly honored to watch her grow as a scientist over four years at Caltech. She spearheaded the binuclear cobaloxime catalyst project (Chapter 5), helped lead the Blair High School SHArK program, and is hands-down one of the best all-around undergraduates I ever met at Caltech. I am excited that we will be colleagues at the University of Washington and know she will have an incredibly successful graduate career. Chantal Mustoe prepared ligands for silicon surface tethering (Chapter 6) and I am grateful for her dedication to this project. She is an incredibly diligent and curious chemist, and it has been a pleasure to work with her. I am happy that she will continue this work independently after my graduation. Kedy Edme prepared the cobaloxime catalysts with the olefin modified glyoxime ligands and performed the homogeneous 
electrochemical investigations presented in Chapter 6. I have never met a student with as much self-motivation and sincere curiosity as Kedy, and I appreciate his patience with me during my home-stretch thesis work this past summer. I know he has a bright future ahead of him and look forward to hearing all about his achievements. Gates Winkler spent a tireless summer retrofitting a diode array spectrometer and writing software for the nanosecond-II system, which was invaluable to this thesis research. In addition to these contributions, he brought a great sense of humor to the lab and revealed that his dad does actually like to have fun. Lastly, Eve Ra worked on designing new reductive quenchers, and I am grateful for her hard work and cheerfulness.

It has been a pleasure to work among many great researchers in the Gray Group, who I consider both friends and colleagues. First, Charlotte Whited and I quickly became the best of friends, and I am grateful for the many adventures we have had over the last four years in and out of the lab. I am confident that we will remain lifelong friends and cannot imagine my time at Caltech without her. Alec Durrell made me laugh almost every day and shared my enthusiasm for time-resolved spectroscopy. I have learned a great deal about spectroscopy from our experiences together as laser GLAs, and he advised me on a number of experiments contained in this thesis. Gretchen Keller has been a great source of support over the last five years, and I will miss her sarcasm, party-planning, and partying. Matthew Hartings is both a great friend and an incredible mentor, and I look forward to having great scientific conversations and sharing good recipes with him for many years to come. In addition, I have enjoyed spending time with his wonderful family, who remind me of the value of balance in life. Kyle Lancaster has epitomized all the great stereotypes of grad students: he is hard-working, awesome when he's angry, and knows how to tie one on. Crystal Shih, Bert Lai, Yen Hoang Le Nguyen, and Bryan Stubbert have all been great office mates and our office conversations have been a great respite from the daily work in lab. 
Further, Crystal (and Lina) was a great roommate and I thank her for teaching me the many ins and outs of graduate school. James McKone helped bring the SHArK program to Pasadena, and taught me a great deal about photoelectrochemistry. Brian Leigh and Don Walker introduced me to all the equipment in BILRC and showed me where to get lunch first year. Maraia Ener also shares my enthusiasm for spectroscopy and kinetics and gives awesome group meetings. Lionel Cheruzel brought French culture to the lab and is an incredibly dedicated researcher. Morgan Cable, Paul Oblad, Matt Bierman, Paul Bracher, and Jeff Warren are all great scientists, gave me great advice at some point or another, and have made significant contributions to the lab atmosphere. Lastly, there are a number of other outstanding graduate students, undergrads, and postdocs who I had the privilege to overlap with: Peter Agbo, Gitrada Arjara, Nicole Bouley, Rose Bustos, Steve Contakes, Megan Jackson, Hema Karunadasa, Tetsu Kimura, Yan Choi Lam, Judy Lattimer, Katie Lavoie, Jennifer Lee, John Magyar, Qixi Mi, Astrid Mueller, Josh Palmer, Kate Pletneva, Mike Rose, Kevin Sea, Karn Sorasaenee, Heather Williamson, Melanie Yen, and Keiko Yokoyama.

The staff at Caltech is fabulous, and these people made the last five years run very smoothly. In particular I would like to acknowledge Joe Drew, Pat Anderson, Catherine May, Rick Jackson, Elyse Garlock, Rick Gerhart, Tom Dunn, Rick Germond, Art Seiden, Larry Martinez, Paul Carroad, Scott Ross, David Vander Velde, Mona Shahgholi, Naseem Torian, Anne Penney, Agnes Tong, Laura Howe, Dian Buchness, Terry James, Ron Koen, Cora Carriedo, Carlos, Victor, and Jose for all their hard work and dedication to the department.

Outside of lab, a number of friends have helped make Caltech a great place to go to graduate school. Mary Laura Lind and I ran hundreds of miles together, chatting through every single one of them. She is a great friend and has dragged me out of lab to enjoy many beautiful days, shown me some great places in California to hike, and makes a mean 
chocolate stout cake. Nilay Hazari is perhaps the most sincere, generous, and noble person I have ever met; he has challenged me to be a better person and I am lucky to have him as my "best friend." Caroline Saouma and I came to Caltech together after becoming friends during our senior year at MIT. We shared a great travel adventure together in Europe before hunkering down for grad school, and she taught me that the best way to deal with stress is to bake delicious things.

John Bercaw and his lab have provided a great second "lab away from lab," and I am lucky to have so many great friends in the group, including Suzanne Golisz, Valerie Scott, Steve Baldwin, Ian Tonks, Rachel Klet, and Dave Weinberg. I would also like to acknowledge a number of other great friends in the department who have contributed to my graduate experiences, including Eric Olmon, Leslie O’Leary, Maddy Radlauer, Matt Whited, Arjun Mendiratta, and the rest of the "Crystallography Group," Katy Muzikar and Sean Kedrowski.

I fell in love with inorganic chemistry during my UROP in the Nocera group at MIT, and chose to attend graduate school because of this experience. I am grateful to all the graduate students and postdocs who trained and inspired me to pursue my interests in chemistry especially Becky Somers, Steve Reece, Arthur Esswein, Bart Bartlett, Dave Manke, Amy Prieto, and Matt Shores. Many of these friends have continued to advise and support me through graduate school, and I am thankful for their guidance.

Beyond Caltech, Sally Smith, Stavroula Hatzios, and Dominique Pradella have all been the best of friends and put up with my failure to return phone calls in a timely manner. The TOGs of the Los Feliz Flyers Running Club have been great track buddies and remind me weekly of all that lies beyond Caltech. 
I would not be where I am today without my family, who encouraged me to pursue my dreams from a very young age. My parents have supported me from afar throughout my time at Caltech. They have selflessly encouraged me to pursue this degree, despite outrage from coworkers for "letting me move to California." I love them very dearly and could not have done this without them. Elizabeth and Scott have been great siblings and friends, despite living 2700 miles away. In addition, I want to thank my grandfather who has supported my interest in science throughout the years and let me tag along to visit the labs he consulted for, experiences which undoubtedly shaped my career decisions.

Lastly, I cannot imagine the past five years without the love of my life, Alex Miller. He is a truly remarkable chemist and has inspired me to be a better scientist. He has helped me in all my synthetic endeavors and made significant intellectual contributions to this thesis. More importantly, he has been by my side throughout the ups and downs of graduate school, trained for and ran a marathon to spend more time with me, and encouraged us to get out of lab and explore the west coast together. I look forward to sharing all of life's adventures that lie ahead with him. 
Cobaloximes are among a promising class of small molecules which catalytically evolve hydrogen at modest overpotentials. Motivated by the imminent need to develop efficient solar energy conversion processes, a number of research groups have recently revisited the catalytic activity of cobaloximes, which was initially reported by Espenson almost three decades ago. Both Espenson's seminal work and the studies reported during this recent resurgence are chronicled in the introductory Chapter 1 . The next three chapters introduce photochemical methods for detecting catalytic intermediates and determining kinetics associated with the elementary steps of hydrogen evolution. Four catalytic pathways are considered, each beginning with the reduction of a $\mathrm{Co}^{\mathrm{II}}$-diglyoxime to generate $\mathrm{Co}^{\mathrm{I}}$, which reacts with a proton donor to produce a $\mathrm{Co}^{\mathrm{III}}$-hydride. In a homolytic pathway, two $\mathrm{Co}^{\mathrm{III}}$ hydrides react in a bimolecular step to eliminate $\mathrm{H}_{2}$. Alternatively, in a heterolytic pathway, protonation of $\mathrm{Co}^{\mathrm{III}}$-hydride produces $\mathrm{H}_{2}$ and $\mathrm{Co}^{\mathrm{III}}$. The $\mathrm{Co}^{\mathrm{III}}$-hydride may also be reduced further to a $\mathrm{Co}^{\mathrm{II}}$-hydride, which can react via analogous heterolytic or homolytic pathways. In Chapter 2, kinetics of electron transfer reactions of a Co-diglyoxime complex are presented. These experimental results, along with a detailed thermodynamic analysis of proposed hydrogen evolution pathways, shed new light on the barriers and driving forces of the elementary reaction steps involved in proton reduction. A strong thermodynamic preference for a $\mathrm{Co}^{\mathrm{III}}$-hydride homolytic pathway over a $\mathrm{Co}^{\mathrm{III}}$-hydride heterolytic route is identified as the key finding from this work. In Chapter 3, phototriggered hydride generation utilized in conjunction with time-resolved spectroscopy is introduced as a novel method for mechanistic investigations. Here, excited-state proton transfer from an organic photoacid to a $\mathrm{Co}^{\mathrm{I}}$-diglyoxime triggers the formation of the reactive $\mathrm{Co}^{\mathrm{III}}$-hydride. This and the subsequent reactivity of $\mathrm{Co}^{\mathrm{III}}$-hydride are monitored spectroscopically. The reaction kinetics are consistent with a heterolytic route for hydrogen evolution that proceeds via a 
$\mathrm{Co}^{\mathrm{II}}$-hydride intermediate. Chapter 4 extends these mechanistic investigations to aqueous media by employing photoionization and pulse radiolysis methods to trigger $\mathrm{Co}^{\mathrm{II}}$ diglyoxime reduction. Chapters 5 and 6 focus on the design and construction of secondgeneration cobaloximes. In Chapter 5, the thermodynamic preference for bimolecular reactivity of two $\mathrm{Co}^{\mathrm{III}}$-hydrides is probed with a binuclear cobaloxime. A covalent alkyl tether is used to decrease the volume required for diffusional collisions. Electrocatalytic activity is consistent with a rate-limiting step associated with the formation of the hydride, as seen in mononuclear catalysts, and thus no enhancement of catalytic activity is observed. However, as an efficient water splitting device may require the tethering of catalysts to an electrode surface, this ligand should allow binuclear association of immobilized catalysts. A strategy for covalently grafting cobaloxime derivatives to silicon electrodes is introduced in Chapter 6. A terminal olefin is incorporated into a glyoxime backbone, a functionality amenable to surface-based coupling reactions. The bifunctional cobaloxime is an active catalyst, and initial efforts to prepare the chemically modified electrode are discussed. Three appendices are provided, including work on the photochemical generation of powerful Os ${ }^{\mathrm{II}}$ reductant, electron transfer reactions of $\mathrm{N}, \mathrm{N}^{\prime}, 3,3^{\prime}$-tetramethyl-4,4'-bipyridinium, and annotated MATLAB scripts utilized for kinetics analysis. 
Acknowledgements ...................................................................................... iv

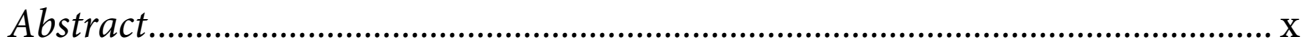

Table of Contents..........................................................................................

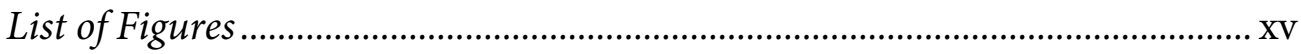

List of Tables ...............................................................................................

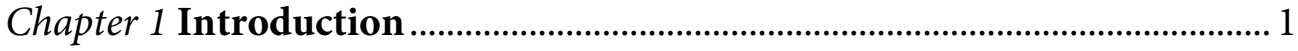

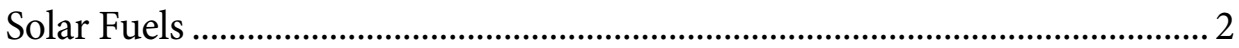

Catalytic $\mathrm{H}_{2}$ Production ............................................................................ 5

Biomimetic Hydrogenase Complexes ............................................................ 6

Other Molecular Catalysts............................................................................

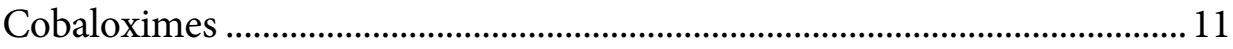

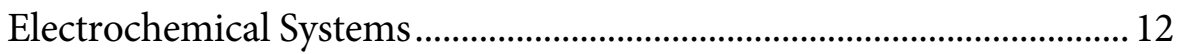

Photochemical Systems ............................................................................. 15

Reaction Pathways …………………………………………………..... 18

Prospects for Cobaloxime Chemistry............................................................2

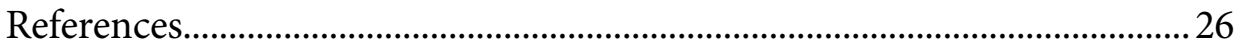

Chapter 2 Pathways of $\mathbf{H}_{2}$ Evolution: Barriers and Driving Forces .............. 33

Introduction .............................................................................................. 34

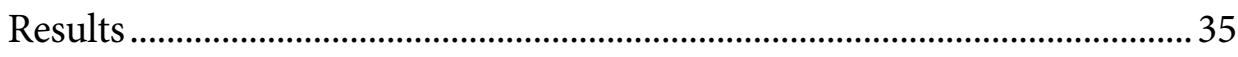

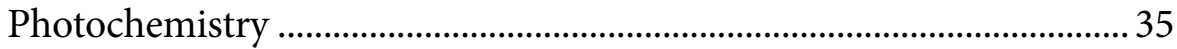

Rate Constants and Reorganization Energies ..........................................4 43

Thermodynamic Analysis.......................................................................... 46

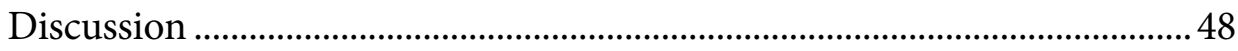

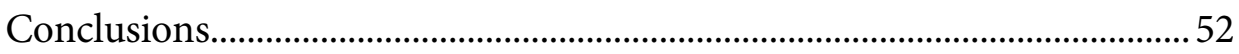

Acknowledgement ........................................................................................ 53

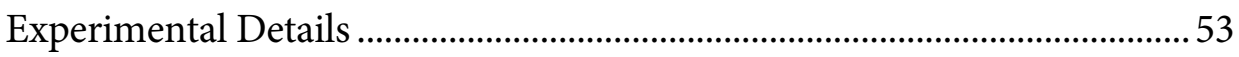

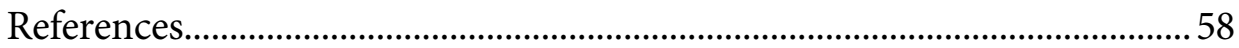

Chapter 3 Mechanism of $\mathbf{H}_{2}$ Evolution from a Phototriggered

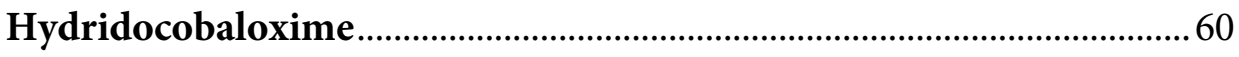

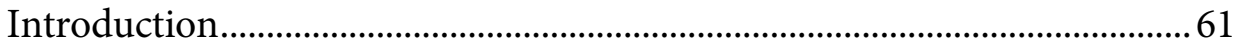

Results and Discussion ................................................................................ 62

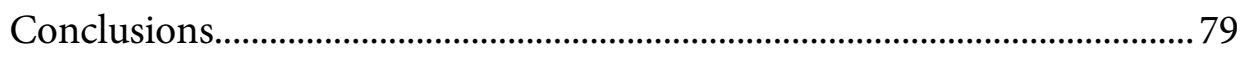

Acknowledgement ......................................................................................... 79

Experimental Details ................................................................................ 


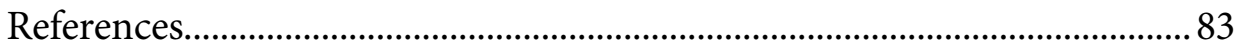

Chapter 4 Mechanistic Investigations of Cobaloxime Catalyzed $\mathbf{H}_{2}$

Evolution in Aqueous Media ………………………………...................... 86

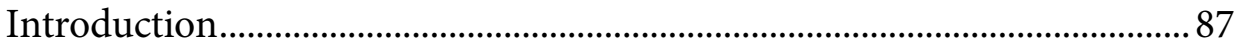

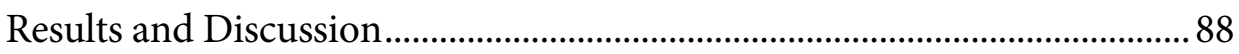

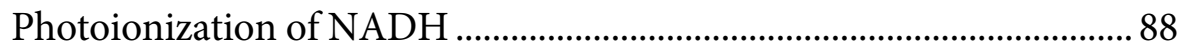

Photoionization of Indole ......................................................................99

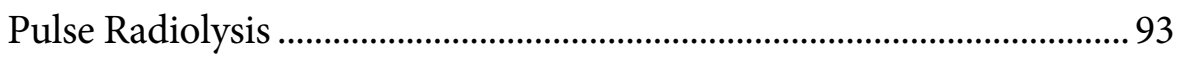

Challenges in Aqueous Mechanistic Studies ............................................ 101

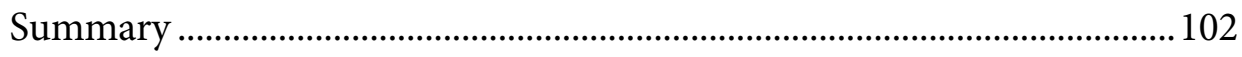

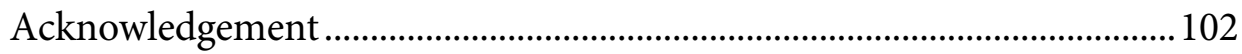

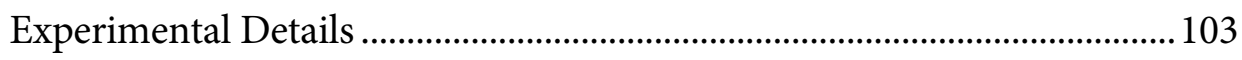

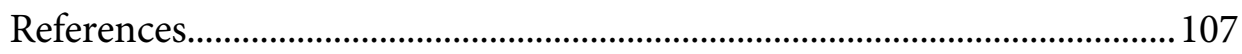

Chapter 5 Catalytic $\mathbf{H}_{2}$ Evolution from a Binuclear Cobaloxime .............. 109

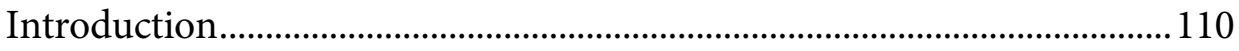

Results and Discussion...............................................................................111

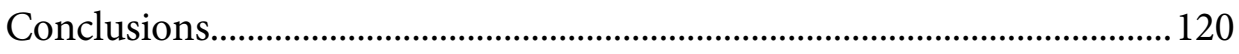

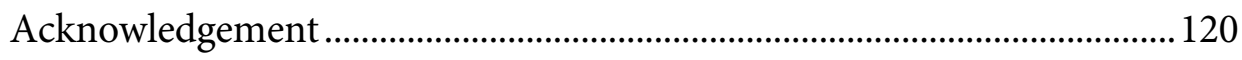

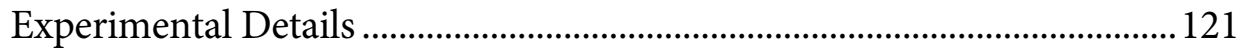

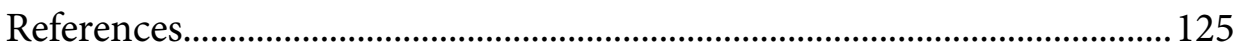

Chapter 6 Bifunctional Cobaloximes for Covalent Attachment to Silicon

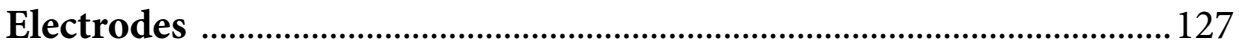

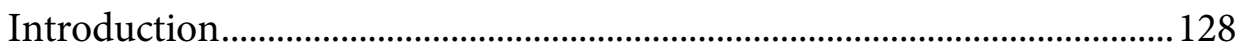

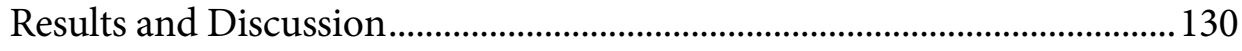

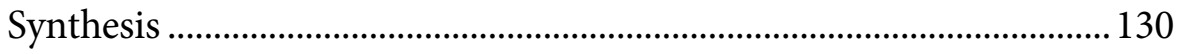

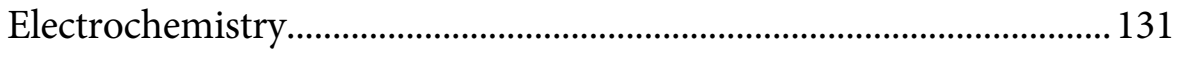

Surface Functionalization ..................................................................... 133

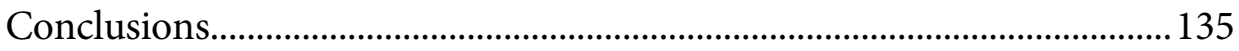

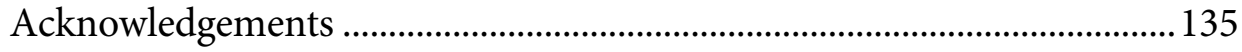

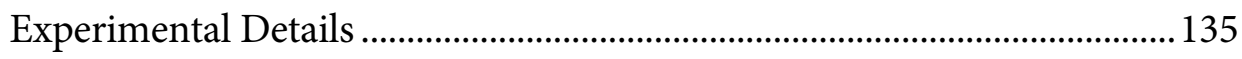

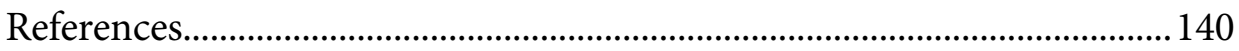

Appendix A Photochemical Generation of a Powerful Os(II) Reductant... 142

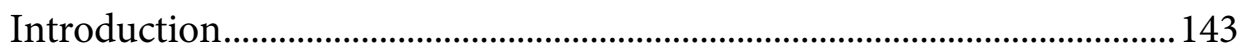

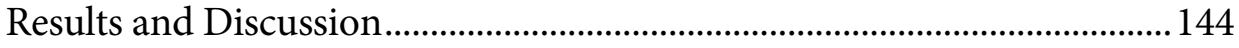

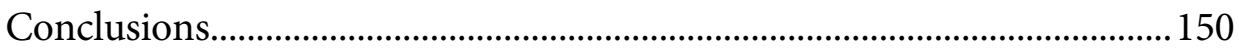

Experimental Details …………………………………………………...... 151

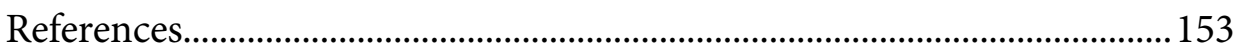


Appendix B Electron Transfer Reactions of N,N',3,3'-Tetramethyl-4,4'-

Bipyridinium ................................................................................................ 155

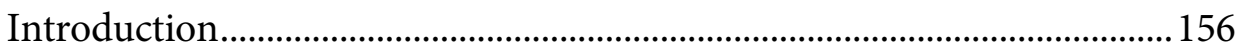

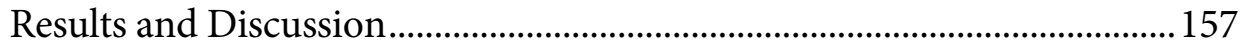

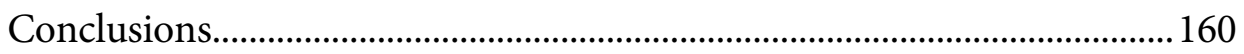

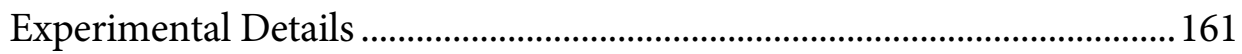

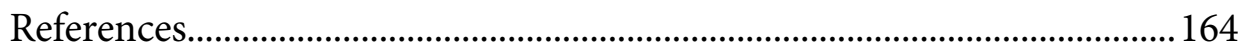

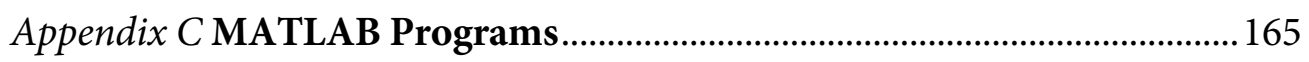




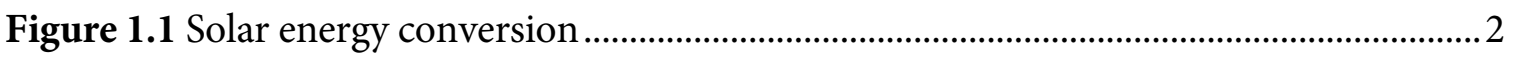

Figure 1.2 A proposed solar-driven water splitting device ....................................................... 4

Figure 1.3 Line drawings of the active site in [NiFe] and [FeFe] hydrogenase.......................... 7

Figure 1.4 A selection of molecular $\mathrm{H}_{2}$ evolving catalysts ....................................................

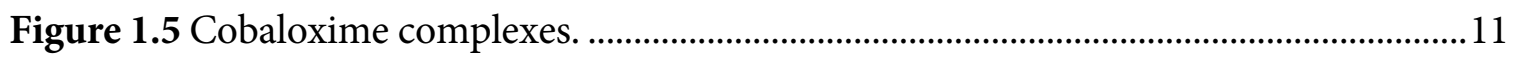

Figure 1.6 Sensitizer-cobaloxime conjugates ..............................................................................17

Figure 2.1 Homolytic and heterolytic reaction pathways for $\mathrm{H}_{2}$ evolution..............................34

Figure 2.2 Photosensitizer, electron relay, and catalyst ........................................................36

Figure 2.3 Transient difference spectra .......................................................................................37

Figure 2.4 Absorption spectra of reactants and intermediates..................................................37

Figure 2.5 Time-resolved transient difference spectra and global analysis fits ......................39

Figure 2.6 Stern-Volmer quenching plot for $\left[\mathrm{Ru}(\mathrm{bpy})_{3}\right]^{2+}$ and $\mathrm{MV}^{2+}$......................................40

Figure 2.7 Kinetics trace of $20 \mu \mathrm{M}\left[\mathrm{Ru}(\mathrm{bpy})_{3}\right]\left[\mathrm{PF}_{6}\right]_{2}, 3.5 \mathrm{mM}[\mathrm{MV}]\left[\mathrm{PF}_{6}\right]_{2}$, and $0.1 \mathrm{M}$ $\mathrm{NBu}_{4} \mathrm{PF}_{6}$ and the simulated fit in logarithmic time..................................................4

Figure 2.8 Kinetics trace of $20 \mu \mathrm{M}\left[\mathrm{Ru}(\mathrm{bpy})_{3}\right]\left[\mathrm{PF}_{6}\right]_{2}, 3.5 \mathrm{mM}[\mathrm{MV}]\left[\mathrm{PF}_{6}\right]_{2}, 0.1 \mathrm{M} \mathrm{NBu}_{4} \mathrm{PF}_{6}$, $125 \mu \mathrm{M} \mathrm{Co}\left(\mathrm{dpgBF}_{2}\right)_{2}\left(\mathrm{CH}_{3} \mathrm{CN}\right)_{2}$, and the simulated fit in logarithmic time ................43

Figure 2.9 Thermodynamic analysis of homolytic and heterolytic pathways .......................46

Figure 3.1 Absorption Spectra of 6-bromo-2-naphtholand sodium 6-bromo-2-naphtholate in acetonitrile.

Figure 3.2 Normalized luminescence spectra of 6-bromo-2-naphthol and sodium 6bromo-2-naphtholate, room temperature, in acetonitrile solution..............................64

Figure 3.3 Normalized luminescence spectra of 6-bromo-2-naphthol and sodium 6bromo-2-naphtholate, $77 \mathrm{~K}$, in butyronitrile glass..........................................................64

Figure 3.4 Normalized phosphorescence spectra of 6-bromo-2-naphthol at $77 \mathrm{~K}$ and at room temperature

Figure 3.5 Normalized luminescence spectra of sodium 6-bromo-2-naphtholate at 77K and at room temperature.

Figure3.6 Absorption Spectra of $\mathrm{Co}(\mathrm{II})\left(\mathrm{dmgBF}_{2}\right)_{2}\left(\mathrm{CH}_{3} \mathrm{CN}\right)_{2}, \quad[\mathrm{Na}]$ $\left.\left[\mathrm{Co}(\mathrm{I})\left(\mathrm{dmgBF}_{2}\right)_{2}\left(\mathrm{CH}_{3} \mathrm{CN}\right)\right], \mathrm{MeCo}(\mathrm{III})\left(\mathrm{dmgBF}_{2}\right)_{2}\left(\mathrm{CH}_{3} \mathrm{CN}\right)\right]$ in acetonitrile..............67

Figure 3.7 Absorption spectra and corresponding global fits .................................................68

Figure 3.8 Photographs of a bulk photolysis sample before and after photolysis ..................69

Figure 3.9 First-order rate constants determined from excited-state quenching of 6-bromo2-naphthol in the presence of $\left[\mathrm{Co}\left(\mathrm{dmgBF}_{2}\right)_{2}\left(\mathrm{CH}_{3} \mathrm{CN}\right)\right]^{-}$. .71 
Figure 3.10 Typical transient kinetics trace for a sample containing $600 \mu \mathrm{M}$ 6-bromo-2naphthol, $50 \mu \mathrm{M}[\mathrm{Na}]\left[\mathrm{Co}^{\mathrm{I}}\left(\mathrm{dmgBF}_{2}\right)_{2}\left(\mathrm{CH}_{3} \mathrm{CN}\right)\right]$ and $100 \mathrm{mM}\left[\mathrm{NBu}_{4}\right]\left[\mathrm{PF}_{6}\right]$ in logarithmic time $\left(\lambda_{\text {obs }}=630 \mathrm{~nm}\right)$.

Figure 3.11 First-order rate constants for bleaching $\left[\mathrm{Co}\left(\mathrm{dmgBF}_{2}\right)_{2}\left(\mathrm{CH}_{3} \mathrm{CN}\right)\right]^{-}$absorption

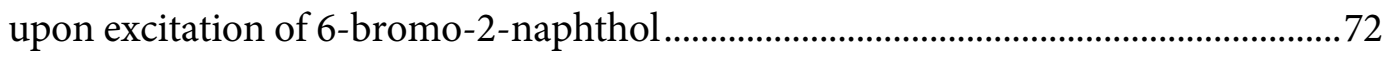

Figure 3.12 Energy cycle based on the Förster cycle ..........................................................73

Figure 3.13 Kinetics traces monitoring the absorption of ${ }^{3 *}$ [6-bromo-2-naphthol] with

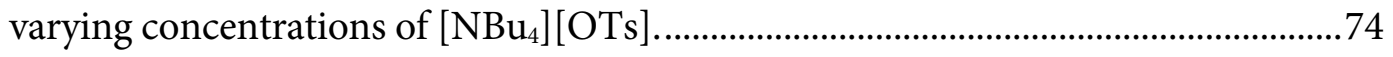

Figure 3.14 Rate constants for the biexponential decay of ${ }^{3^{*}}$ [6-bromo-2-naphthol] in the

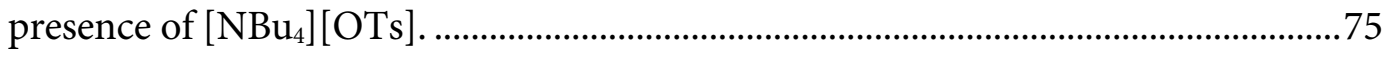

Figure 3.15 The slow kinetics process of kinetics traces measured at $630 \mathrm{~nm}$ fit to a biexponential decay.. .76

Figure 3.16 Typical transient kinetics trace at $405 \mathrm{~nm}$ for a sample containing $583 \mu \mathrm{M} 6$ bromo-2-naphthol, $263 \mu \mathrm{M} \quad[\mathrm{Na}]\left[\mathrm{Co}^{\mathrm{I}}\left(\mathrm{dmgBF}_{2}\right)_{2}\left(\mathrm{CH}_{3} \mathrm{CN}\right)\right]$ and $100 \mathrm{mM}$ $\left[\mathrm{NBu}_{4}\right]\left[\mathrm{PF}_{6}\right]$

Figure 4.1 Cyclic voltammograms (current $(\mu \mathrm{A})$ vs. Potential (V vs. SCE)) recorded for $\mathrm{Co}\left(\mathrm{dmgBF}_{2}\right)_{2}\left(\mathrm{H}_{2} \mathrm{O}\right)_{2}$

Figure 4.2 Kinetics trace observed at $630 \mathrm{~nm}$ for the reaction of $\mathrm{Co}\left(\mathrm{dmgBF}_{2}\right)_{2}\left(\mathrm{H}_{2} \mathrm{O}\right)_{2}+\mathrm{e}_{\mathrm{aq}}{ }^{-}$ formed via photoionization of $\mathrm{NADH}$, fit to first-order exponential $\left(k_{\mathrm{obs}}=9.4 \times 10^{3}\right.$ $\left.\mathrm{s}^{-1}\right)$

Figure 4.3 Transient difference spectra at $\mathrm{pH} 7$ for the reaction of $\mathrm{Co}(\mathrm{dmgBF})_{2}\left(\mathrm{H}_{2} \mathrm{O}\right)_{2}$ with $e_{\mathrm{aq}}{ }^{-}$, formed via photoionization of indole.

Figure 4.4 Transient difference spectra at $\mathrm{pH} 3$ for the reaction of $\mathrm{Co}\left(\mathrm{dmgBF}_{2}\right)_{2}\left(\mathrm{H}_{2} \mathrm{O}\right)_{2}$ with $e_{\mathrm{aq}}{ }^{-}$, formed via photoionization of indole.

Figure 4.5 Difference spectra for the reaction of $\mathrm{Co}\left(\mathrm{dmgBF}_{2}\right)_{2}\left(\mathrm{H}_{2} \mathrm{O}\right)_{2}$ with $e_{\text {aq }}{ }^{-}$,after radiolysis pulse at $\mathrm{pH} 4$ and 8 ..

Figure 4.6 Observed first-order rate of $\mathrm{Co}\left(\mathrm{dmgBF}_{2}\right)_{2}\left(\mathrm{H}_{2} \mathrm{O}\right)_{2}$ reduction by $e_{\mathrm{aq}}{ }^{-}$......................95

Figure 4.7 Difference spectra for the reaction of $\mathrm{Co}\left(\mathrm{dmgBF}_{2}\right)_{2}\left(\mathrm{H}_{2} \mathrm{O}\right)_{2}$ with $\mathrm{CO}_{2}{ }^{-{ }^{-}}$-...............97

Figure 4.8 Observed first-order rate of $\mathrm{Co}\left(\mathrm{dmgBF}_{2}\right)_{2}\left(\mathrm{H}_{2} \mathrm{O}\right)_{2}$ reduction by $\mathrm{CO}_{2}{ }^{{ }^{-}}{ }^{-}$..................97

Figure 4.9 Absolute spectrum of $\mathrm{Co}\left(\mathrm{dmgBF}_{2}\right)_{2}\left(\mathrm{H}_{2} \mathrm{O}\right)_{2}+\mathrm{H} \bullet$...............................................99

Figure 4.10 Kinetics trace and simulation observed at $465 \mathrm{~nm}$ for the reaction of $\mathrm{Co}\left(\mathrm{dmgBF}_{2}\right)_{2}\left(\mathrm{H}_{2} \mathrm{O}\right)_{2}+\bullet^{t} \mathrm{BuOH}$. 100

Figure 4.11 Absolute spectrum of $\mathrm{Co}\left(\mathrm{dmgBF}_{2}\right)_{2}\left(\mathrm{H}_{2} \mathrm{O}\right)_{2}+{ }^{t} \mathrm{BuOH}$.........................................101

Figure 5.1 Binuclear (5.2) and mononuclear (5.3) cobaloximes ..........................................112

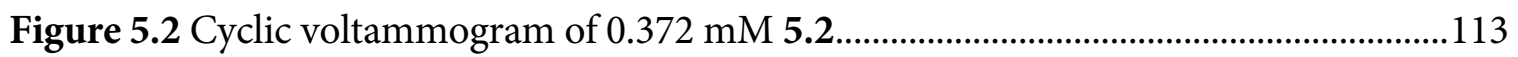

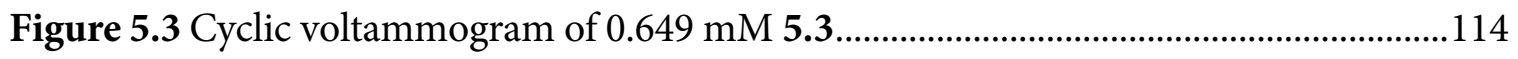

Figure 5.4 Cyclic voltammograms of $0.372 \mathrm{mM} 5.2$ at varying scan rates............................115 
Figure 5.5 Cyclic voltammograms of $0.649 \mathrm{mM} 5.2$ at varying scan rates 115

Figure 5.6 Cyclic voltammogram of $0.372 \mathrm{mM} 5.2$ in the presence of $p$-toluene sulfonic acid monohydrate.

Figure 5.7 Cyclic voltammogram of $0.524 \mathrm{mM} \mathrm{5.3}$ in the presence of $p$-toluene sulfonic acid monohydrate.

Figure 5.8 Dependence of catalytic plateau current on concentration of catalyst 5.2 ........118

Figure 5.9 Dependence of catalytic plateau current on concentration of catalyst $5.3 \ldots \ldots . . .118$

Figure 6.1 Cyclic voltammogram of $0.61 \mathrm{mM} \mathrm{ClCo}^{\mathrm{III}}(\mathrm{pmgH})_{2} \mathrm{py}$

Figure 6.2 Cyclic voltammogram of $0.61 \mathrm{mM} \mathrm{ClCoIII}(\mathrm{pmgH})_{2} \mathrm{py}$ in the absence and presence of $1 \mathrm{mM}\left[\mathrm{Et}_{3} \mathrm{~N}\right][\mathrm{OTs}]$.

Figure 6.3 Cyclic voltammograms of $0.61 \mathrm{mM} \mathrm{ClCo}^{\mathrm{III}}(\mathrm{pmgH})_{2}$ py in the presence of $\left[\mathrm{Et}_{3} \mathrm{~N}\right][\mathrm{OTs}]$.

Figure A.1 Absorption spectra measured at arbitrary intervals upon photolysis 144

Figure A.2 Transient kinetics trace monitoring the appearance of $\mathrm{MV}^{*+}$ upon photochemical generation of $\left[\mathrm{Os}\left(\mathrm{NH}_{3}\right)_{5}\left(\mathrm{H}_{2} \mathrm{O}\right)\right]^{2+}$

Figure A.3 First-order rate constants for the formation of $\mathrm{MV}^{\bullet+}$ upon electron transfer from $\left[\mathrm{Os}\left(\mathrm{NH}_{3}\right)_{5}\left(\mathrm{H}_{2} \mathrm{O}\right)\right]^{2+}$ to $\mathrm{MV}^{2+}$ at $\mathrm{pH} 3$.

Figure A.4 First-order rate constants for the formation of $\mathrm{MV}^{\bullet+}$ upon electron transfer from $\left[\mathrm{Os}\left(\mathrm{NH}_{3}\right)_{5}(\mathrm{OH})\right]^{+}$to $\mathrm{MV}^{2+}$ at $\mathrm{pH} 12$..

Figure A.5 First-order rate constants for the formation of $\mathrm{MV}^{*+}$ upon electron transfer from $\left[\mathrm{Os}\left(\mathrm{NH}_{3}\right)_{5}(\mathrm{OH})\right]^{+}$to $\mathrm{MV}^{2+}$ at $\mathrm{pH} 7$......

Figure A.6 Absorption spectra measured at arbitrary intervals upon photolysis at $355 \mathrm{~nm}$ in acetonitrile..

Figure A.7 First-order rate constants for the formation of $\mathrm{MV}^{\bullet+}$ upon electron transfer from $\left[\mathrm{Os}\left(\mathrm{NH}_{3}\right)_{5}\left(\mathrm{CH}_{3} \mathrm{CN}\right)\right]^{2+}$ to $\mathrm{MV}^{2+}$ in acetonitrile.

Figure B.1 Photosensitizer and quencher. 156

Figure B.2 Synthetic preparation of $\left[{ }^{\mathrm{Me}} \mathrm{MV}\right]\left[\mathrm{PF}_{6}\right]_{2}$

Figure B.3 Cyclic voltammetry of $1.3 \mathrm{mM}\left[{ }^{\mathrm{Me}} \mathrm{MV}\right]\left[\mathrm{PF}_{6}\right]_{2}$. 158

Figure B.4 Spectroelectrochemical measurements of ${ }^{\mathrm{Me}} \mathrm{MV}^{2+}$ 159

Figure B.5 Stern-Volmer quenching plot for the quenching of $\left[\mathrm{Ru}\left({ }^{\mathrm{Me}} \mathrm{bpy}\right)_{3}\right]^{2+*}$ by ${ }^{\mathrm{Me}} \mathrm{MV}^{2+} 160$ 


\section{LIST OF TABLES}

Table $1.1 \mathrm{p} K_{\mathrm{a}}$ values and standard potentials for reduction of acids in acetonitrile ................ 6

Table 1.2 Electrochemical potentials (V vs. SCE in acetonitrile) ............................................13

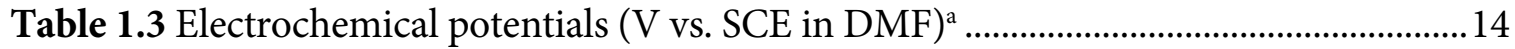

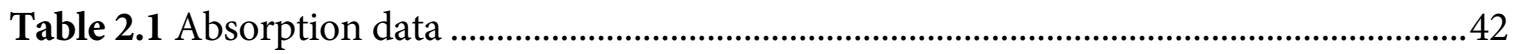

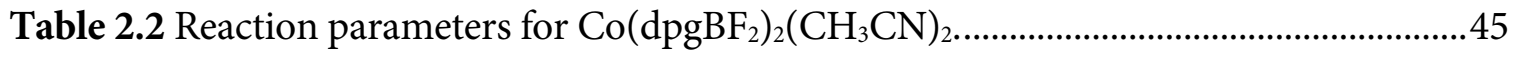

Table 2.3 Reduction potentials (V. vs SCE) in acetonitrile .......................................................45

Table 2.4 Barriers and driving forces associated with $\mathrm{H}_{2}$ evolution .......................................47

Table 3.1 Absorption and fluorescence maxima in $\mathrm{CH}_{3} \mathrm{CN}$ at room temperature................66

Table 3.2 Phosphorescence data in butyronitrile at 77K .........................................................66

Table 3.3 Excited state $\mathrm{p} K_{\mathrm{a}}$ values in $\mathrm{CH}_{3} \mathrm{CN}$ estimated from the Förster cycle.....................66

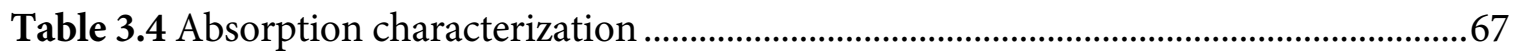

Table 3.5 Sample components for each absorption measurements in Figure 3.7, obtained

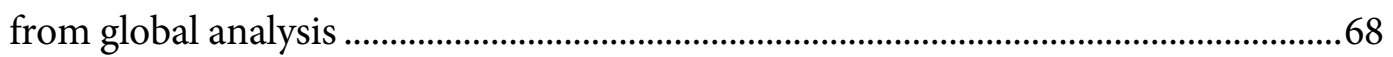

Table 3.6 Hydrogen evolution yields from bulk photolysis experiments ...............................69

Table 5.1 Reduction potentials, apparent rate constants, and diffusion coefficients for

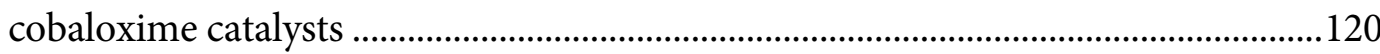


Chapter 1

\section{INTRODUCTION}

Portions of this work are excerpted with permission from:

Dempsey, J. L.; Brunschwig, B. S.; Winkler, J. R.; Gray, H. B. Acc. Chem. Res. 2009, 42, 1995-2004.

Copyright 2009 American Chemical Society 


\section{Solar Fuels}

In response to the current global energy crisis, scientists are going to great lengths to develop renewable resources capable of meeting projected energy demands. ${ }^{1-2}$ Solar energy conversion is an area of enormous promise; using sunlight to make "solar fuels" such as $\mathrm{H}_{2}$ (from $\mathrm{H}_{2} \mathrm{O}$ splitting, Figure 1.1) and $\mathrm{CH}_{3} \mathrm{OH}$ (by reducing $\mathrm{CO}_{2}$ ) is one of the holy grails of $21^{\text {st }}$ century chemistry. ${ }^{3-4}$ Splitting water is highly desirable, as $4.92 \mathrm{eV}$ is stored when $2 \mathrm{H}_{2} \mathrm{O}$ molecules are split into $2 \mathrm{H}_{2}+\mathrm{O}_{2}$, but running the reaction is challenging; it involves two separate multielectron redox processes, a 4-electron oxidation and a 2-electron reduction. ${ }^{5}$ As sequential electron or hole transfers directly to $\mathrm{H}_{2} \mathrm{O}$ produce extremely high energy intermediates, an energy efficient process requires catalysts that avoid formation of these species. ${ }^{6-7}$ Coordination to metals in some cases can stabilize these energetic intermediates, thereby lowering the barriers for hydrogen evolution. ${ }^{8}$

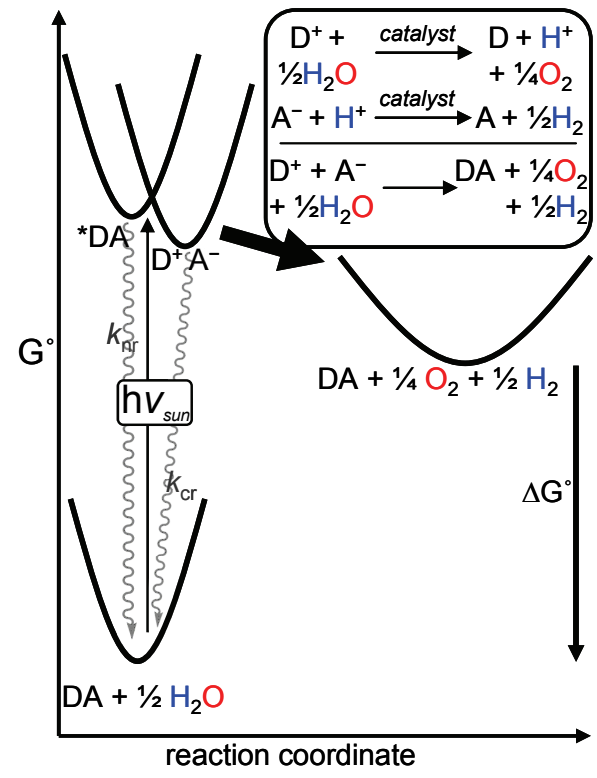

Figure 1.1 Solar energy conversion is initiated by photoexcitation $\left(h v_{\text {sun }}\right)$ of $\mathrm{DA}$ to ${ }^{*} \mathrm{DA}$, which undergoes excited-state electron transfer to yield a charge-separated state $\left(\mathrm{D}^{+} \mathrm{A}^{-}\right)$. $\mathrm{D}^{+}$and $\mathrm{A}^{-}$drive water oxidation and proton reduction via catalysts. 
In green plants, water splitting reactions are carried out by Photosystem II, and the reducing equivalents produced are ultimately stored in energy-rich sugars. Many solardriven water splitting devices aiming to mimic nature's ability to use sunlight to convert abundant energy-poor molecules to energy-rich molecules have been proposed. ${ }^{1,3}$ Notably, an integrated, monolithic photoelectrochemical photovoltaic device that operates with a hydrogen production efficiency of $12.4 \%$ was reported by Turner in $1998 .^{9}$ While this device demonstrates that water splitting can be carried out in synthetic devices at efficiencies far greater than those achieved in nature, it is based on a $\mathrm{GaInP}_{2} / \mathrm{GaAs}$ tandemjunction electrode and uses a platinum catalyst, materials far too rare to be considered in a viable renewable energy device.

Inspired by the great success of Turner and the urgent need to address the global energy crisis, the Powering the Planet Center for Chemical Innovation (CCI-Solar) was formed to develop an efficient and economical solar-driven water splitting device based on earthabundant materials (Figure 1.2). The proposed device is based on a dual-array of microstructured semiconductors embedded in a membrane with both electron- and proton-conducting capabilities. ${ }^{3}$ Upon absorption of blue light, the photoanode will interact with catalysts decorating its surface to oxidize water. The remaining red light will be absorbed by the photocathode material, where catalysts decorating the semiconductor surface will facilitate reduction of protons to hydrogen. The microstructured rod-like architecture is central to the economics of this device. Planar crystalline Si is the basis for most modern photovoltaic devices and a promising photocathode material for this proposed water splitting device, but extremely high purity (and thus expensive) materials are necessary to obtain minority-carrier diffusion lengths of similar magnitude to the optical absorption depth $(\sim 200 \mu \mathrm{m})$ needed to excite silicon's indirect band gap. ${ }^{10-11}$ However, a rod-based geometry permits light absorption and minority-carrier diffusion to 
occur along orthogonal directions; optical absorption occurs along the length of the rod, while minority-carriers are collected at radial junctions. ${ }^{12-13}$ With the short diffusion lengths engendered by radial geometry, the silicon purity requirements are relaxed.

Significant progress has been made towards developing the individual components of this proposed device over the six years since the inception of CCI-Solar research center. As the materials and catalysts necessary for this device continue to be developed and refined, efforts to integrate the different components are now underway. Still, a considerable amount of fundamental chemistry must still be developed before a solar-driven water splitting device based on earth-abundant materials can be fully realized. This thesis focuses on cobaloxime catalysts capable of evolving $\mathrm{H}_{2}$ at low overpotentials, detailing experimental efforts aimed at elucidating the mechanism of efficient $\mathrm{H}_{2}$ evolution, the development of second-generation catalysts, and discussing early experiments designed to integrate the catalyst and photocathode components.

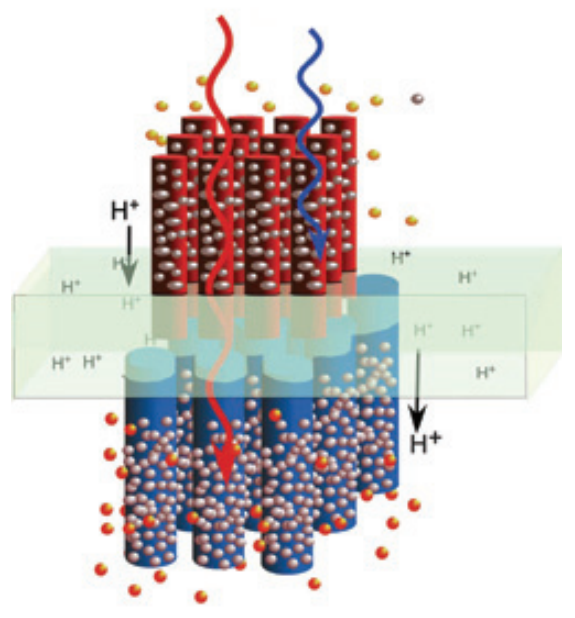

Figure 1.2 A proposed solar-driven water splitting device based on microrod-structured semiconductors (that act as light absorbers and charge separators) embedded in a proton- and electron-conducting membrane. Catalysts anchored to the photocathode and photoanode catalyze the evolution of hydrogen and oxygen, respectively. 


\section{Catalytic $\mathrm{H}_{2}$ Production}

The best catalyst for hydrogen evolution is undeniably platinum, which operates at the thermodynamic potential for $\mathrm{H}^{+} / \mathrm{H}_{2}$ conversion $(0 \mathrm{~V}$ vs. SHE, $\mathrm{pH} 0) .{ }^{14}$ However, the scarcity of this material limits its use in a scalable device. In response, attention has turned to the highly active $[\mathrm{FeFe}],[\mathrm{NiFe}]$, and $[\mathrm{Fe}]$ hydrogenases that catalyze hydrogen oxidation and hydrogen production reactions at molecular active sites embedded in a protein under moderate conditions. ${ }^{15-17}$ Significant efforts have been put forth to develop molecular systems that mimic both the structure and function of these organometallic active sites occurring in nature, discussed in detail below. ${ }^{8,18-20}$

Although the ultimate objective is to use water as a feedstock, ${ }^{21-27}$ many potential $\mathrm{H}_{2}$ evolving catalysts are tested for catalytic activity in organic solvents, as they often are not soluble or stable in aqueous environments. The electrocatalytic behavior of proton sources in organic solvents is markedly different from that in aqueous environments. In nonaqueous solvents, the standard potential for hydrogen evolution, $E_{\mathrm{HA}^{\prime} \mathrm{H}_{2}}^{0}$, is directly related to acid strength, and can be described by

$E_{\mathrm{HA} / \mathrm{H}_{2}}^{0}=E_{\mathrm{H}^{+} / \mathrm{H}_{2}}^{0}-(2.303 R T / F) p K_{\mathrm{a}, \mathrm{HA}}$

where $E_{\mathrm{H}^{+} / \mathrm{H}_{2}}^{0}$ is the solvated proton/dihydrogen couple standard potential in the given solvent and $K_{\mathrm{a}, \mathrm{HA}}$ is the acid dissociation constant for the proton source, determined from a self-consistent scale of acidity in a given solvent. ${ }^{28-30}$ The standard potentials for hydrogen evolution from a variety of commonly used acids is given in Table 1.1. The overpotential for proton reduction is the difference between the potential of catalytic activity and $E_{\mathrm{HA} / \mathrm{H}_{2}}^{0}$; catalysis at potentials more positive than $E_{\mathrm{HA} / \mathrm{H}_{2}}^{0}$ is not expected. Lastly, it must be noted that the standard potentials for hydrogen evolution in organic solvents are very sensitive to 
water. Trace water has been shown to significantly influence the relative strengths of acids via selective solvation processes; efforts have been made to carry out the analytical experiments discussed in rigorously dry solvent. ${ }^{31}$

Table 1.1 $\mathrm{p} K_{\mathrm{a}}$ values and standard potentials for reduction of acids in acetonitrile.

\begin{tabular}{lll}
\hline Acid & $\mathrm{pK}_{a}^{\mathrm{a}}$ & $E_{\mathrm{HA} / \mathrm{H} 2}^{\circ}(\mathrm{V} \text { vs. SCE })^{\mathrm{c}}$ \\
\hline $\mathrm{HBF}_{4} \cdot \mathrm{Et}_{2} \mathrm{O}$ & $0.1^{\mathrm{b}}$ & 0.23 \\
Trifluoromethanesulfonic acid & 2.6 & 0.09 \\
$p$-cyanoanilinium & 7.6 & -0.21 \\
$\mathrm{TsOH} \cdot \mathrm{H}_{2} \mathrm{O}$ & $8.0^{\mathrm{b}}$ & -0.23 \\
$\mathrm{HCl} \cdot \mathrm{Et}_{2} \mathrm{O}$ & $8.9^{\mathrm{b}}$ & -0.29 \\
$\mathrm{CF}_{3} \mathrm{COOH}$ & $12.7^{\mathrm{b}}$ & -0.51 \\
{$\left[\mathrm{Et}_{3} \mathrm{NH}\right]^{+}$} & 18.7 & -0.86 \\
{$\left[\mathrm{Et}_{3} \mathrm{NH}\right]^{+}$} & $9.2(\mathrm{DMF})$ & -0.84 \\
$\mathrm{Acetic} \mathrm{acid}^{2}$ & $13.2(\mathrm{DMF})$ & -1.08 \\
\hline
\end{tabular}

${ }^{\mathrm{a}}$ Reference 28 unless noted otherwise. ${ }^{\mathrm{b}}$ Reference $32{ }^{\mathrm{c}} E_{\mathrm{HA} / \mathrm{H} 2}^{\circ}=E_{\mathrm{H}+\mathrm{H} 2}^{\circ}-0.059 \mathrm{p} K_{a}(\mathrm{HA})$. A potential of $0.24 \mathrm{~V}$ vs. SCE was chosen as the thermodynamic potential for $E_{\mathrm{H}+\mathrm{H} 2}^{\circ}$ in acetonitrile (Reference $33)$.

\section{Biomimetic Hydrogenase Complexes}

The active site structures of the $[\mathrm{NiFe}],[\mathrm{FeFe}]$, and $[\mathrm{Fe}]$ hydrogenases have inspired a great deal of work on synthetic metallobiomolecules aimed at mimicking hydrogen evolution and oxidation functionalities (Figure 1.3). ${ }^{16-19,34}$ Despite the hundreds of structural models developed to date, few have been reported to show electrocatalytic activity related to proton reduction or hydrogen oxidation. While upwards of 50 structural mimics of [NiFe] hydrogenases have been reported, initially only biomimetic complexes that deviated 
significantly from the enzyme structure displayed any electrocatalytic behavior, including a trinuclear $\left\{\mathrm{Ni}^{\prime} \mathrm{S}_{2}{ }^{\prime} \mathrm{Fe}_{2}\right\}$ system ${ }^{35-36}$ and a few $\mathrm{Ni}-\mathrm{Ru}$ complexes ${ }^{37-42}$ prepared in the laboratories of Schroder, Fontecave, and Ogo. ${ }^{43}$ More recently though, two competent $\mathrm{Ni}$ Fe catalysts were reported that mimic both the structure and function of the active hydrogenase..$^{44-45}$

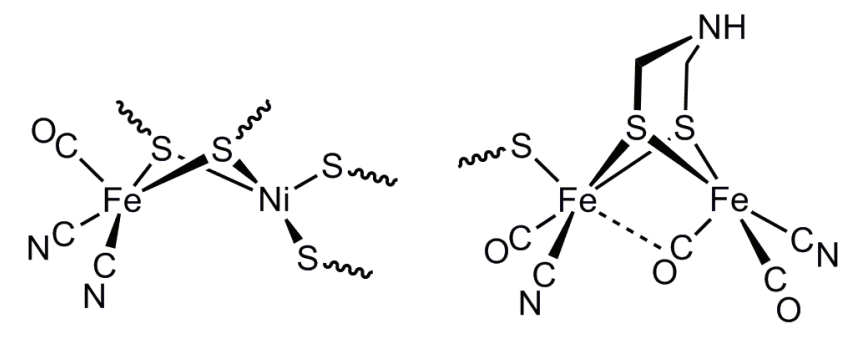

Figure 1.3 Line drawings of the active site in [NiFe] hydrogenase and $[\mathrm{FeFe}]$ hydrogenase. $\mathrm{S} \sim$ = cysteine residue.

A good deal more [FeFe] hydrogenase mimics have been reported. ${ }^{46-47}$ Rauchfuss and coworkers reported the first di-iron dithiolate system, $\left\{\mathrm{Fe}_{2}\left[\mu-\mathrm{S}_{2}\left(\mathrm{CH}_{2}\right)_{3}\right](\mathrm{CN})(\mathrm{CO})_{4}\left(\mathrm{PMe}_{3}\right)\right\}^{-}$, which evolved hydrogen catalytically (at $-1.13 \mathrm{~V}$ vs. $\mathrm{Ag} / \mathrm{AgCl}$ in $\mathrm{CH}_{3} \mathrm{CN}$ ) in $2001 .{ }^{48}$ While the efficiency of this system is far removed from that of the natural hydrogenase, it nevertheless demonstrated successful biomimetic function. In the wake of this seminal work, many related systems displaying electrocatalytic properties have since been reported. ${ }^{18,49}$ Most, however, operate at relatively large overpotentials. The models have, however, afforded significant insight into the roles of bridging thiolate ligands and the hydride binding modes associated with catalysis. ${ }^{19}$

\section{Other Molecular Catalysts}

A number of non-biomimetic systems show remarkable efficiency for $\mathrm{H}_{2}$ evolution (Figure 1.4). The most noteworthy examples are molecular $\mathrm{Co}-, \mathrm{Ni}-$, and Mo-based systems which 
evolve hydrogen efficiently without the use of noble metals. Cobalt complexes with diglyoxime ligands (cobaloximes) is one notable class of catalysts that evolves hydrogen at low overpotentials; the hydrogen evolution chemistry of these catalysts will be highlighted in the subsequent section.

A mononuclear nickel complex with non-coordinating pendent amines incorporated into the backbone of cyclic diphosphine ligands demonstrates high activity for catalytic

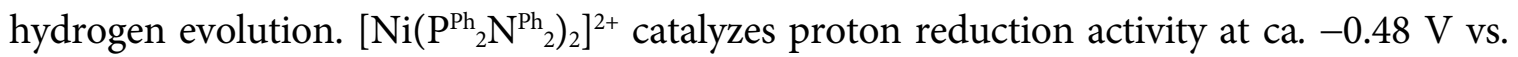
SCE with trifluoromethanesulfonic acid or protonated dimethylformamide, corresponding overpotentials of 0.57 and $0.35 \mathrm{~V}$, respectively. ${ }^{50-52}$ Under optimal conditions, a turnover frequency of $350 \mathrm{~s}^{-1}$ was reported, comparable to those of [NiFe] hydrogenases. Extensive mechanistic studies have indicated that the pendent bases act to both stabilize dihydrogen and relay protons to the metal site, and this functionality in the second coordination sphere leads to much higher rates and lower overpotentials as compared to analogous complexes lacking these bases. ${ }^{52-53}$ Notably, similar nickel complexes with 0 or 2 pendent nitrogen bases display activity for the reverse reaction, hydrogen oxidation, emphasizing the proximity of the operating potential to the thermodynamic potential for $\mathrm{H}_{2}$ evolution. ${ }^{54}$ 

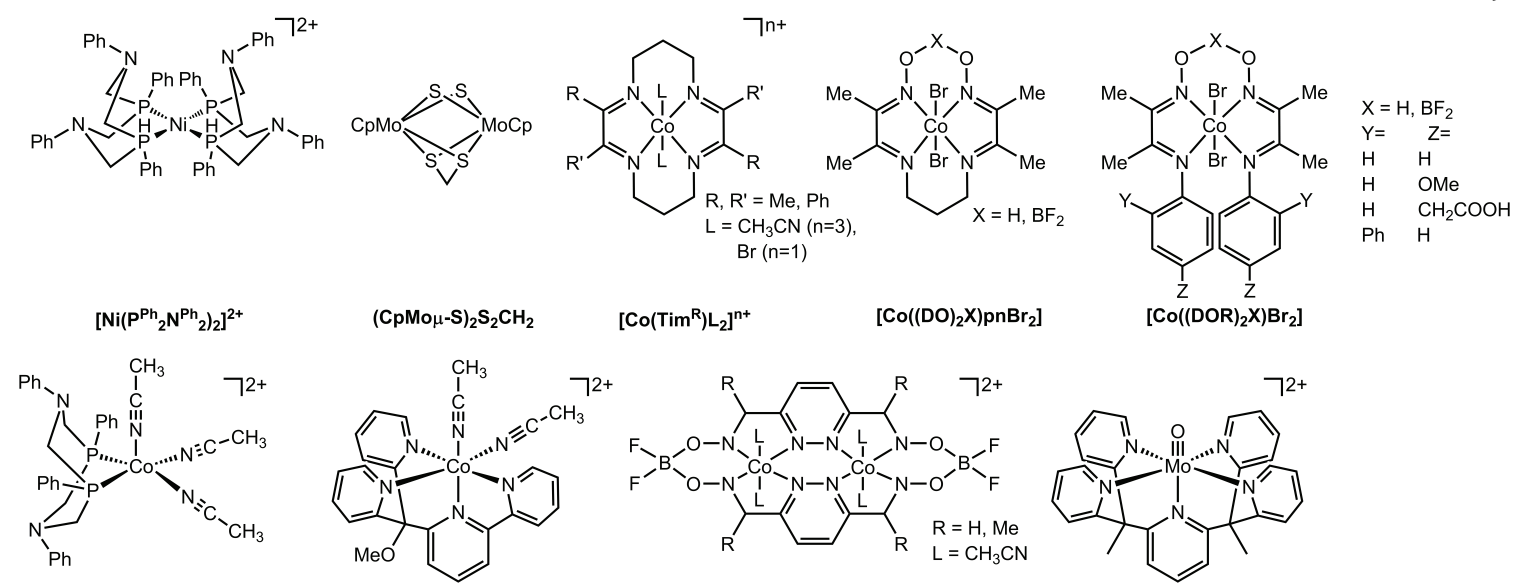

$\left[\mathrm{Co}\left(\mathrm{PPh}_{2} \mathrm{~N}^{\mathrm{Ph}}\right)\left(\mathrm{CH}_{3} \mathrm{CN}\right)_{3}\right]^{2+}$

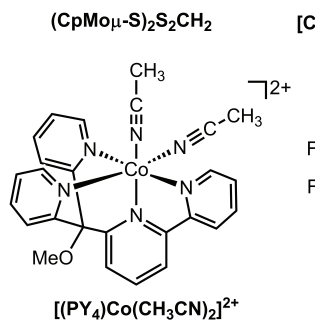

$\left[\mathrm{Co}\left(\operatorname{Tim}^{R}\right) L_{2}\right]^{n+}$

$\left[\mathrm{Co}\left((\mathrm{DO})_{2} \mathrm{X}\right) \mathrm{pnBr}_{2}\right]$

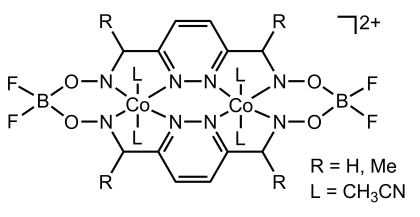

$\left[\mathrm{L}^{\mathrm{R}} \mathrm{Co}_{2}\right]^{2+}$

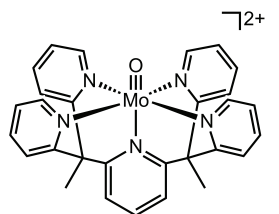

$\left[\left(\mathrm{PY}_{5} \mathrm{Me}_{2}\right) \mathrm{MoO}^{2+}\right.$

Figure 1.4 A selection of molecular $\mathrm{H}_{2}$ evolving catalysts.

A molybdenum-based system (CpMou-S $)_{2} \mathrm{~S}_{2} \mathrm{CH}_{2}$ is a tremendously efficient electrocatalyst, operating at a $50 \mathrm{mV}$ overpotential with ( $p$-cyanoanilinium) $\mathrm{BF}_{4}$ as a proton source (at $-0.26 \mathrm{~V}$ vs. SCE in acetonitrile). ${ }^{55}$ Notably, hydrogen evolution is thought to be centered at the bridging sulfur atoms, not the metal centers.

Several tetraimine complexes have been prepared that exhibit similar structure and catalytic activity to the cobaloximes of interest. Noteworthy among several cobalt complexes supported by [14]-tetraene- $\mathrm{N}_{4}$ ligands are the methyl substituted analogues $\left[\mathrm{Co}\left(\mathrm{Tim}^{\mathrm{Me}}\right) \mathrm{Br}_{2}\right]^{+}$and $\left[\mathrm{Co}\left(\mathrm{Tim}^{\mathrm{Me}}\right)\left(\mathrm{CH}_{3} \mathrm{CN}\right)_{2}\right]^{3+}$ which display catalytic peak currents at the corresponding $\mathrm{Co}^{\mathrm{II} / \mathrm{I}}$ potentials, occurring at -0.35 and $-0.38 \mathrm{~V}$ vs. SCE, respectively, with near Faradaic yields. ${ }^{33}$ Hybrid dioxime diimine complexes containing one propyl linkage and one oxime linkage, $\left[\mathrm{Co}\left((\mathrm{DO})_{2} \mathrm{X}\right) \mathrm{pnBr}_{2}\right]\left(\mathrm{X}=\mathrm{H}, \mathrm{BF}_{2}\right)$, also demonstrate catalytic activity near their respective $\mathrm{Co}^{\mathrm{II} / \mathrm{I}}$ potentials $(-0.73 \mathrm{~V}$ vs. SCE for the proton linked complex and $-0.46 \mathrm{~V}$ for the $\mathrm{BF}_{2}$ linkage) with near Faradaic yields. ${ }^{56} \mathrm{~A}$ variety of semi-cyclic complexes supported by $\mathrm{N}$-aryl Shiff base/oxime ligands with either proton or $\mathrm{BF}_{2}$ linkages, $\left[\mathrm{Co}\left((\mathrm{DOR})_{2} \mathrm{X}\right) \mathrm{Br}_{2}\right]\left(\mathrm{X}=\mathrm{H}, \mathrm{BF}_{2}\right)$, are active catalysts, with catalytic reduction potentials 
occurring between -0.48 and $-0.75 \mathrm{~V}$ vs. SCE, depending on the aryl substituent. ${ }^{57}$ Notably, the carboxylic acid substituted complex was adsorbed onto ITO surfaces and some catalytic behavior was observed in presence of acid, though ultimately the acidic conditions promoted dissociation of the catalyst from the surface.

In addition to these imine and oxime based catalysts, many other cobalt based systems have recently been reported to catalyze hydrogen evolution. ${ }^{58}$ Three recent reports are worth detailing here. A cobalt complex containing a single cyclic diphosphine ligand, $\left[\mathrm{Co}\left(\mathrm{P}_{2}{ }_{2} \mathrm{~N}^{\mathrm{Ph}}\right)\left(\mathrm{CH}_{3} \mathrm{CN}\right)_{3}\right]^{2+}$, is an active electrocatalyst, operating with bromoanilinium tetrafluoroborate at a moderate overpotential of $258 \mathrm{mV}$, achieving turnover frequency of $90 \mathrm{~s}^{-1} \cdot{ }^{59}$ A cobalt polypyridyl complex, $\left[\left(\mathrm{PY}_{4}\right) \mathrm{Co}\left(\mathrm{CH}_{3} \mathrm{CN}\right)_{2}\right]^{2+} \quad\left(\mathrm{PY}_{4}=\right.$ 2-bis $(2-$ pyridyl)(methoxy)methyl-6-pyridylpyridine), has been shown to catalyze the reduction of trifluoroacetic acid at the $\mathrm{Co}^{\mathrm{II} / \mathrm{I}}$ reduction potential, $-0.81 \mathrm{~V}$ vs. SCE, corresponding to an overpotential of $c a .400 \mathrm{mV} .{ }^{60} \mathrm{~A}$ dicobalt macrocycle, $\left[\mathrm{L}^{\mathrm{R}} \mathrm{Co}_{2}\right]^{2+}$, evolves $\mathrm{H}_{2}$ upon addition of 2,6-dichloroanilinium tetrafluoroborate at $-0.3 \mathrm{~V}$ and $-0.7 \mathrm{~V}$ vs. SCE for the $\mathrm{H}$ - and $\mathrm{CH}_{3}$ - substituted complexes, potentials cathodic of the $\mathrm{Co}^{\mathrm{II}} \mathrm{Co}^{\mathrm{I}} / \mathrm{Co}^{\mathrm{I}} \mathrm{Co}^{\mathrm{I}}$ redox couple and corresponding to overpotentials of -0.24 and $-0.64 \mathrm{~V}$, respectively. In this system, bimetallic cooperativity is thought to be important for the catalytic function.

Only a few molecular catalysts have been reported that produce $\mathrm{H}_{2}$ from water, including $\mathrm{Ni}(\text { cyclam })^{2+},{ }^{22} \mathrm{Co}(\text { bpy })_{3}{ }^{2+}$ (bpy $=2,2^{\prime}$-bipyridine $),{ }^{24}$ as well as several water soluble $\mathrm{Co}^{\mathrm{II}}$ (porphyrin) complexes ${ }^{21}$ and other cobalt(II) complexes with nitrogen-based macrocycles, ${ }^{61-63}$ including a cobaloxime central to this thesis, $\mathrm{Co}\left(\mathrm{dmgBF}_{2}\right)_{2}\left(\mathrm{H}_{2} \mathrm{O}\right)_{2}\left(\mathrm{dmgBF}_{2}\right.$ $=$ difluoroboryl-dimethylglyoxime, $\left.\left[\mathrm{Co}^{\mathrm{II}}\right]\right){ }^{20,64}$ Among recently reported catalysts, $\left[\left(\mathrm{PY}_{5} \mathrm{Me}_{2}\right) \mathrm{MoO}^{2+}\left(\mathrm{PY} 5 \mathrm{Me}_{2}=2,6\right.\right.$-bis(1,1-bis(2-pyridyl)ethyl)pyridine) is notable for its ability to produce $\mathrm{H}_{2}$ from water. ${ }^{65}$ The molybdenum-oxo complex operates at an 
overpotential of $0.52 \mathrm{~V}$ in $0.6 \mathrm{M}$ phosphate buffer $(\mathrm{pH} 7)$, and under optimal conditions $\left(-1.4 \mathrm{~V}\right.$ vs. SHE) achieves a turnover frequency of 2.4 moles $\mathrm{H}_{2}$ per mole of catalyst per second and a turnover number of $6.1 \times 10^{5}$ moles of $\mathrm{H}_{2}$ per mole of catalyst. A proposed catalytic cycle suggests that proton reduction is a ligand centered process.

\section{Cobaloximes}

Cobaloximes are a promising class of molecules which evolve hydrogen in both aqueous and organic media at relatively low overpotentials (Figure 1.5). ${ }^{20}$ They are further attractive due to the relative ease of their preparation. Extensive chemical, electrochemical, and photochemical studies have focused on characterizing their $\mathrm{H}_{2}$ evolution catalysis, yet many questions remain as to the mechanism of the $\mathrm{H}_{2}$ evolving reaction. Further, little work has been done to prepare modified glyoxime ligands, which could enhance catalytic behavior and provide additional functionality desirable for a solar water splitting device.
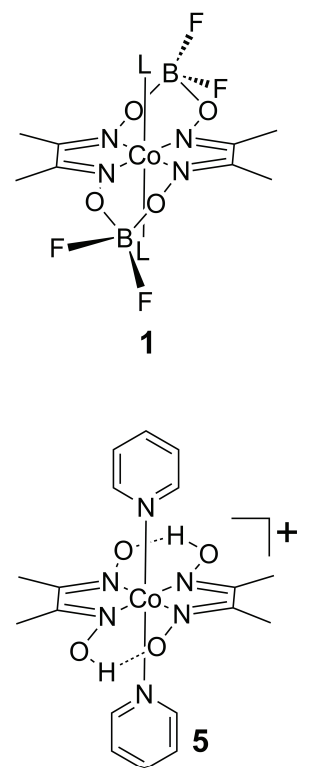
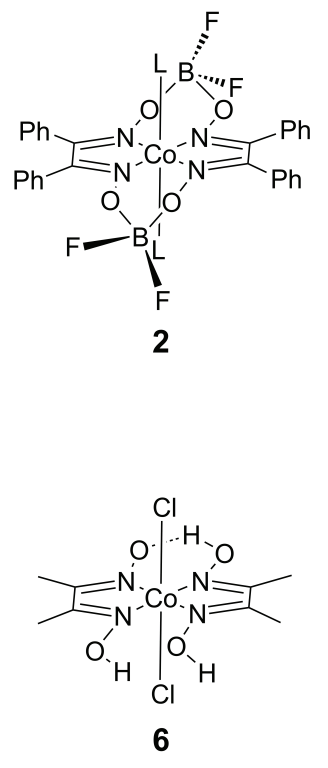

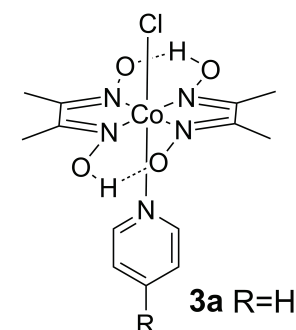

3b $\mathrm{R}=\mathrm{NMe}_{2}$ 3c $\mathrm{R}=\mathrm{COOMe}$

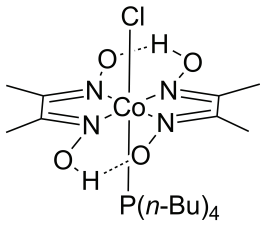

7

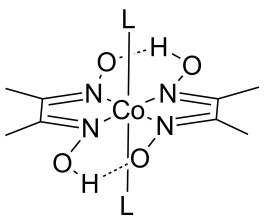

4

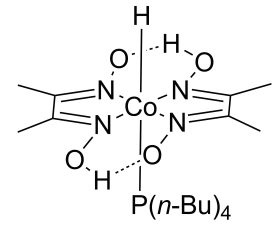

7H

Figure 1.5 Cobaloxime complexes. $\mathrm{L}$ is typically $\mathrm{H}_{2} \mathrm{O}$ or $\mathrm{CH}_{3} \mathrm{CN}$ 
Electrochemical Systems

Connolly and Espenson first reported that $\mathrm{Co}\left(\mathrm{dmgBF}_{2}\right)_{2}(\mathrm{~L})_{2}\left(\mathbf{1}, \mathrm{dmgBF}_{2}=\right.$ difluoroboryldimethylglyoxime) catalyzes the reduction of $\mathrm{HCl}_{\mathrm{aq}}$ using $\mathrm{Cr}^{2+}{ }_{\mathrm{aq}}$ as a stoichiometric electron donor. $^{64}$ Dissociation of an intermediate chloro-bridged complex, $\left[\left(\mathrm{H}_{2} \mathrm{O}\right)_{5} \mathrm{Cr}-\mathrm{Cl}\right.$ $\left.\mathrm{Co}(\mathrm{dmgBF})_{2}\right]^{+}$, which was formed during inner-sphere electron transfer from $\mathrm{Cr}^{\mathrm{II}}$ to $\mathrm{Co}^{\mathrm{II}}$, produces $\left[\mathrm{Co}\left(\mathrm{dmgBF}_{2}\right)_{2} \mathrm{~L}\right]^{-}\left(\mathbf{1}^{-}\right)$. In the presence of acid, it is likely that the $\mathrm{Co}^{\mathrm{I}}$ anion is rapidly protonated to form a hydride, $\left[\mathrm{HCo}\left(\mathrm{dmgBF}_{2}\right)_{2} \mathrm{~L}\right]$, which evolves $\mathrm{H}_{2}$.

Complex 1 and a related species, $\operatorname{Co}\left(\operatorname{dpgBF}_{2}\right)_{2}(\mathrm{~L})_{2} \quad(2, \mathrm{dpg}=$ difluoroboryldiphenylglyoxime), ${ }^{66}$ were later shown electrochemically to catalyze $\mathrm{H}_{2}$ evolution in acetonitrile. ${ }^{67-68}$ The reversible, one-electron reduction of 1 occurs at $-0.55 \mathrm{~V}$ vs. SCE in acetonitrile (Table 1.2). Upon addition of a sufficiently strong acid, catalytic currents were observed near the $\mathrm{Co}^{\mathrm{II} / \mathrm{I}}$ couple. Increasing acid concentration produced an increase in peak current, a slight positive shift in peak position, and loss of the return oxidation wave, with the current eventually approaching a plateau. Since $\mathrm{Co}^{\mathrm{II}}$ is regenerated during $\mathrm{H}_{2}$ production, there is no return oxidation wave. Bulk electrolysis experiments confirmed near quantitative Faradaic yields of $\mathrm{H}_{2}$. A range of proton sources was examined: catalysis was observed with $\mathrm{CF}_{3} \mathrm{COOH}, \mathrm{HCl} \cdot \mathrm{Et}_{2} \mathrm{O}, p$-toluenesulfonic acid monohydrate $\left(\mathrm{TsOH} \bullet \mathrm{H}_{2} \mathrm{O}\right),{ }^{33}$ ( $p$-cyanoanilinium) $\mathrm{BF}_{4}$, and $\mathrm{HBF}_{4} \bullet \mathrm{Et}_{2} \mathrm{O}$. Although $\mathrm{HBF}_{4} \bullet \mathrm{Et}_{2} \mathrm{O}$ was shown to effect $\mathrm{H}_{2}$ evolution with substantially increased rates, competitive degradation pathways of the catalyst greatly limited reaction efficiencies. 
Table 1.2 Electrochemical potentials (V vs. SCE in acetonitrile).

\begin{tabular}{llll}
\hline Complex & $E^{\circ}\left(\mathrm{Co}^{\mathrm{III} / \mathrm{II}}\right)$ & $E^{\circ}\left(\mathrm{Co}^{\mathrm{II} / \mathrm{I}}\right)$ & Reference \\
\hline $\mathbf{1}$ & $\sim 0.2^{\mathrm{a}}$ & -0.55 & 67 \\
$\mathbf{2}$ & $\sim 0.3^{\mathrm{a}}$ & -0.28 & 67 \\
$\mathbf{3 a}$ & -0.68 & -1.13 & $69^{\mathrm{b}}$ \\
$3 \mathbf{b}$ & -0.84 & -1.13 & $69^{\mathrm{b}}$ \\
$3 \mathbf{c}$ & -0.78 & -1.09 & $69^{\mathrm{b}}$ \\
$\mathbf{5}$ & -0.39 & -1.1 & $69^{\mathrm{b}}$ \\
$\mathbf{6}$ & -0.49 & -0.8 & $69^{\mathrm{b}}$ \\
7 & -0.92 & -0.99 & $69^{\mathrm{b}}$ \\
\hline
\end{tabular}

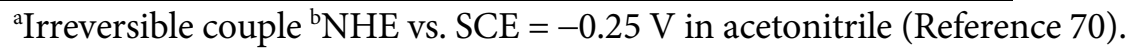

Substitution of methyl groups by electron withdrawing phenyl substituents to form $\mathbf{2}$ shifts the $\mathrm{Co}^{\mathrm{II} / \mathrm{I}}$ reduction potential $270 \mathrm{mV}$ positive to $-0.28 \mathrm{~V}$ vs. SCE. Electrocatalysis was observed with $\mathrm{HCl} \cdot \mathrm{Et}_{2} \mathrm{O}$, (p-cyanoanilinium) $\mathrm{BF}_{4}, \mathrm{TsOH} \bullet \mathrm{H}_{2} \mathrm{O}$, and $\mathrm{HBF}_{4} \bullet \mathrm{Et}_{2} \mathrm{O}$, but not $\mathrm{CF}_{3} \mathrm{COOH}$, with currents reaching a plateau at high acid concentrations.

Studies of cobalt difluoroboryl-bridged diglyoximes as well as related compounds with [14]-tetraene- $\mathrm{N}_{4}$ ligands led to a correlation between catalyst activity $\left(\mathrm{H}_{2}\right.$ evolution) and $\mathrm{Co}^{\mathrm{II} / \mathrm{I}}$ potentials. ${ }^{33}$ Complexes with more negative reduction potentials were able to catalyze proton reduction with weaker acids at higher rates than those with more positive reduction potentials. Metal hydride $\mathrm{p} K_{\mathrm{a}}$ values increase as the $\mathrm{Co}^{\mathrm{II} / \mathrm{I}}$ reduction potentials become more negative; thus the $\mathrm{Co}^{\mathrm{II} / \mathrm{I}}$ potentials are related to $\mathrm{Co}^{\mathrm{I}}$ basicities. ${ }^{71}$ 
The overpotentials for proton reduction catalyzed by cobaloxime complexes can be estimated from the difference between $E_{\mathrm{HA} / \mathrm{H}_{2}}^{0}$ and the potential where catalytic behavior is observed, which occurs just negative of $E^{0}\left(\mathrm{Co}^{\mathrm{II} / \mathrm{I}}\right)$. Catalysts that reduce protons near the thermodynamic potential also should oxidize $\mathrm{H}_{2}$ in the presence of a conjugate base. Indeed, slow oxidation of $\mathrm{H}_{2}$ by $\mathbf{1}$ in the presence of $\left[\mathrm{NBu}_{4}\right]\left[\mathrm{CF}_{3} \mathrm{CO}_{2}\right]$ has been observed. The $\mathrm{H}_{2}$ evolution overpotential was calculated to be $90 \mathrm{mV}$ based on the reaction equilibrium constant. ${ }^{33}$

Table 1.3 Electrochemical potentials (V vs. SCE in DMF). ${ }^{\mathrm{a}}$

\begin{tabular}{|c|c|c|c|}
\hline Complex & $E^{\circ}\left(\mathrm{Co}^{\mathrm{III} / \mathrm{II}}\right)$ & $E^{\circ}\left(\mathrm{Co}^{\mathrm{II} / \mathrm{I}}\right)$ & Reference \\
\hline $3 a$ & -0.67 & -1.06 & 72 \\
\hline $3 b$ & -0.74 & -1.09 & 72 \\
\hline 4 & & -1.06 & 72 \\
\hline 5 & -0.32 & -1.06 & 72 \\
\hline 7 & -0.70 & -0.89 & 72 \\
\hline \multicolumn{4}{|c|}{$\begin{array}{l}\text { a Converted to } \mathrm{SCE} \text { from } \mathrm{Ag} / \mathrm{AgCl} / 3 \mathrm{~mol} \mathrm{~L} \mathrm{~L}^{-1} \mathrm{NaCl}, \mathrm{Fc}^{+} / \mathrm{Fc} \text { vs. } \\
\mathrm{Ag} / \mathrm{AgCl}=0.55 \mathrm{~V} \text { in } \mathrm{DMF} \text { (Reference } 72), \mathrm{Fc}^{+} / \mathrm{Fc} \text { vs. } \mathrm{SCE}=0.47 \mathrm{~V} \\
\text { in } \mathrm{DMF} \text { (Reference } 73 \text { ). }\end{array}$} \\
\hline
\end{tabular}

The electrocatalytic behavior of similar cobalt glyoximes also has been examined. $\mathrm{Co}(\mathrm{dmgH})_{2} \mathrm{pyCl}(3 \mathbf{a}, \mathrm{py}=$ pyridine $)$ catalyzes $\mathrm{H}_{2}$ evolution at a $\mathrm{Co}^{\mathrm{II} / \mathrm{I}}$ potential of $-1.06 \mathrm{~V}$ vs. SCE in DMF with $\left[\mathrm{Et}_{3} \mathrm{NH}^{+}\right] \mathrm{Cl}$ (Table 1.3). ${ }^{72}$ Upon reduction to the $\mathrm{Co}^{\mathrm{II}}$ species, the halide ligand is labilized, while the axial pyridine stays coordinated. Substitution of the axial pyridine for 4-(dimethylamino)pyridine (3b) does not substantially modify the $\mathrm{Co}^{\mathrm{II} / \mathrm{I}}$ potential, but the catalyst appears to be more electroactive. 
$\mathrm{Co}(\mathrm{dmgH})_{2} \mathrm{~L}_{2}(4)$ has the same $\mathrm{Co}^{\mathrm{II} / \mathrm{I}}$ reduction potential as 3 in $\mathrm{DMF}^{72}$ The glyoximebridging $\mathrm{BF}_{2}$ groups, produced via reaction of the complex with $\mathrm{BF}_{3} \bullet \mathrm{Et}_{2} \mathrm{O},{ }^{74}$ shift $\mathrm{Co}^{\mathrm{II} / \mathrm{I}}$ potentials to values $\sim 0.5 \mathrm{~V}$ more positive than their corresponding hydrogen-bridged counterparts, but require stronger acids for electrocatalytic hydrogen evolution. The bridging $\mathrm{BF}_{2}$ groups also increase the stability of the catalysts in acidic solutions.

\section{Photochemical Systems}

Catalytic hydrogen production can be driven directly with light when a sacrificial electron donor is present to scavenge the oxidized photosensitizer. ${ }^{75-76}$ Photosensitizers can act both as light harvesters and photoreductants; quenching of their electronic excited states by electron transfer, directly to a catalyst or via an electron mediator, generates the reduced catalyst. Ziessel and coworkers demonstrated a multicomponent photochemical system for homogeneous $\mathrm{H}_{2}$ generation with a cobaloxime catalyst in organic solvents, utilizing $\left[\mathrm{Ru}(\mathrm{bpy})_{3}\right]^{2+}\left(\right.$ bpy $=2,2^{\prime}$-bipyridine $)$ as photosensitizer, triethanolamine (TEOA) as sacrificial electron donor, and 4 as catalyst. ${ }^{77}$ In DMF solution at $\mathrm{pH} 8.8$, the turnover number for $1 \mathrm{~h}$ of irradiation (based on photosensitizer concentration) was 38 .

Among other work of note, ${ }^{78}$ Eisenberg and coworkers have shown that $\mathbf{3 a}$ catalyzes $\mathrm{H}_{2}$ evolution with a platinum(II) terpyridyl phenylacetylide complex, $[\mathrm{Pt}(\operatorname{ttpy})(\mathrm{C} \equiv \mathrm{CPh})]^{+}(\mathrm{ttpy}$ $=4^{\prime}$ - $p$-tolylterpyridine), as photosensitizer and TEOA as a donor in 3:2 (v/v) $\mathrm{CH}_{3} \mathrm{CN}: \mathrm{H}_{2} \mathrm{O}$ solutions between $\mathrm{pH} 7$ and $\mathrm{pH} 12 .{ }^{79}$ At $\mathrm{pH} 8.5$ with $0.27 \mathrm{M}$ TEOA, 1000 turnovers were achieved after $10 \mathrm{~h}$ irradiation $(\lambda>410 \mathrm{~nm})$.

Other cobaloxime catalysts with varying axial bases (3a-c, 5-7) and $\mathrm{Pt}(\mathrm{II})$ acetylide photosensitizers have been investigated. ${ }^{69}$ With 3a as catalyst, 2150 turnovers of $\mathrm{H}_{2}$ were achieved after $10 \mathrm{~h}$ irradiation $(\lambda>410 \mathrm{~nm})$ in a $24: 1(\mathrm{v} / \mathrm{v}) \mathrm{CH}_{3} \mathrm{CN}: \mathrm{H}_{2} \mathrm{O}$ mixture at $\mathrm{pH} 8.5$. 
Fontecave and coworkers have focused on multicomponent photosystems for $\mathrm{H}_{2}$ evolution with 1 and both $\left[\operatorname{Ir}(\text { ppy })_{2}(\text { phen })\right]^{+}$(ppy $=2$-phenylpyridine, phen $=$ phenanthroline) and $\left[\operatorname{ReBr}(\mathrm{CO})_{3}(\right.$ phen $\left.)\right]$ as photosensitizers in acetone. Solutions were buffered using $\mathrm{Et}_{3} \mathrm{~N} / \mathrm{Et}_{3} \mathrm{NH}^{+}$, which provided both a sacrificial donor $\left(\mathrm{Et}_{3} \mathrm{~N}\right)$ and a proton source $\left(\mathrm{Et}_{3} \mathrm{NH}^{+}\right) .{ }^{80} \mathrm{~A}$ quantum yield of $16 \pm 1 \%$ and 273 turnovers were achieved with $\left[\operatorname{ReBr}(\mathrm{CO})_{3}(\right.$ phen $\left.)\right]$ in a $15 \mathrm{~h}$ experiment $(\lambda>380 \mathrm{~nm})$ with 600 equivalents each of $\mathrm{Et}_{3} \mathrm{~N}$ and $\mathrm{Et}_{3} \mathrm{NH}^{+}$.

Alberto and coworkers studied a similar photosystem with 4, employing $\left[\operatorname{ReBr}(\mathrm{CO})_{3}(\mathrm{bpy})\right]$ as a photosensitizer, $1 \mathrm{M}$ TEOA as sacrificial electron donor, and $0.1 \mathrm{M}$ acetic acid as a proton source in DMF.${ }^{81} 150$ turnovers in $9 \mathrm{~h}$ irradiation $(\lambda>400 \mathrm{~nm})$ were observed for the system, which had a $26 \pm 2 \%$ quantum yield. Dimethylglyoxime $(7.5 \mathrm{mM})$ was added to enhance catalytic activity, reportedly by countering catalyst degradation pathways. When $\mathrm{Br}^{-}$was replaced by $[\mathrm{NCS}]^{-}$and acetic acid by $[\mathrm{HTEOA}]\left[\mathrm{BF}_{4}\right]$, the long-term stability of the systems is increased significantly and up to 6000 turnovers were achieved. ${ }^{82}$

Systems also have been reported in which organic chromophores replaced noble metal photosensitizers. ${ }^{83-84}$ In $12 \mathrm{~h}$ of irradiation $(\lambda>450 \mathrm{~nm}), 900$ turnovers were achieved using the Eosin $\mathrm{Y}$ photosensitizer with 3a, TEOA, and $3 \mathrm{mM}$ free dimethylglyoxime in 1:1 $\mathrm{CH}_{3} \mathrm{CN}: \mathrm{H}_{2} \mathrm{O}$ at $\mathrm{pH} 7 .{ }^{83}$ Degradation of the photosensitizer/catalyst system was minimized with added dimethylglyoxime, though it was difficult to completely avoid photodecomposition. When a rhodamine dye containing Se in place of $\mathrm{O}$ was utilized as a photosensitizer, turnover numbers reached 9000 in $8 \mathrm{~h}$ of irradiation under similar conditions. ${ }^{84}$ Notably, the turnover frequency achieved in this system is the highest yet reported for the photoreduction of water (5500 $\mathrm{mol} \mathrm{H}_{2} / \mathrm{mol}$ photosensitizer/h, $\Phi=32.8 \%$ ). In a similar noble-metal-free system utilizing Rose Bengal as a photosensitizer and the 
robust catalyst 1, Sun and coworkers noted a turnover number of 327 in 1:2 $\mathrm{CH}_{3} \mathrm{CN}$ at pH 10 with $10 \%$ TEOA in $5 \mathrm{~h}$ of irradiation $(\lambda>400 \mathrm{~nm}) .{ }^{85}$

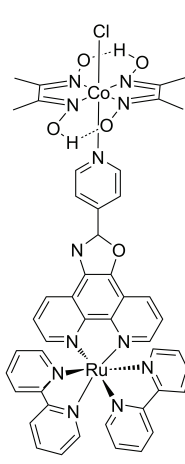

s1
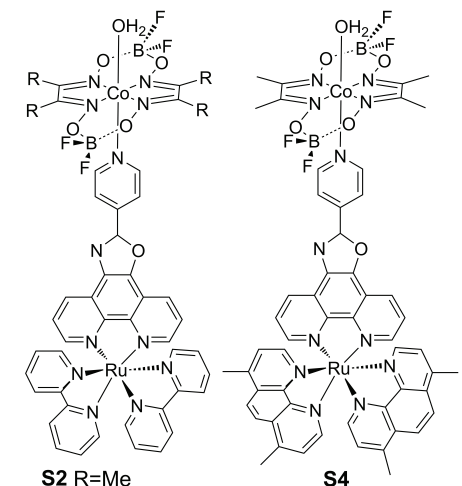

S4

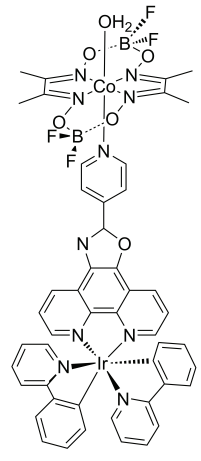

S5

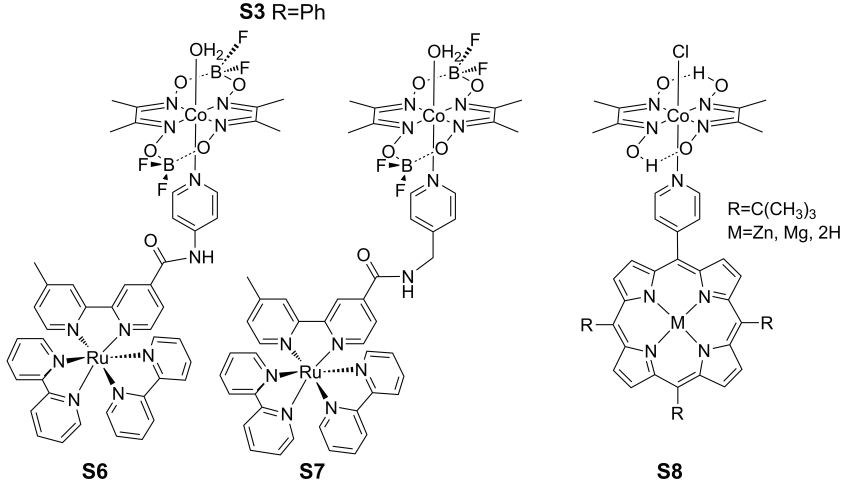

Figure 1.6 Sensitizer-cobaloxime conjugates for photocatalytic hydrogen evolution.

Bifunctional systems (Figure 1.6) have been reported recently with photosensitizers coordinated directly via axial pyridine ligands to $\left[\mathrm{Co}\left(\mathrm{dmgBF}_{2}\right)_{2}\left(\mathrm{H}_{2} \mathrm{O}\right)\right]$ and $\left[\mathrm{Co}(\mathrm{dmgH})_{2} \mathrm{Cl}\right]$ (S1-S5). ${ }^{80,86}$ Irradiation of these sensitizer-catalyst conjugates in $\mathrm{Et}_{3} \mathrm{~N} / \mathrm{Et}_{3} \mathrm{NH}^{+}$buffered acetone solution triggers intramolecular electron transfer from photoexcited ruthenium or iridium sensitizers to the cobalt center, leading to $\mathrm{H}_{2}$ evolution. Notably, these conjugates exhibited efficiencies up to 8.5 times greater than analogous multicomponent systems under the same conditions: $\left[(\text { ppy })_{2} \operatorname{Ir}\left((\mathrm{L}-\mathrm{pyr}) \mathrm{Co}\left(\mathrm{dmgBF}_{2}\right)_{2}\left(\mathrm{H}_{2} \mathrm{O}\right)\right]^{+}(\mathbf{S} 5, \quad\right.$ L-pyr $=(4-$ pyridine)oxazolo[4,5-f]phenanthroline) managed 210 turnovers after $15 \mathrm{~h}$ irradiation with 
600 equivalents of $\mathrm{Et}_{3} \mathrm{~N}$ and $\mathrm{Et}_{3} \mathrm{NH}^{+}$in acetone. $\mathrm{Li}$ et al. studied related heterobinuclear Ru-Co systems with (S6) and without (S7) linker conjugation. ${ }^{87}$ While both complexes were more active than the corresponding multicomponent systems, the one with a nonconjugated bridge exhibited more turnovers. Hydrogen has also been noted in noblemetal-free systems (S8) containing a porphyrin sensitizer with a pyridyl group on the periphery that coordinates to the axial site of the cobaloxime, though the turnovers are not noteworthy. ${ }^{85}$

\section{Reaction Pathways}

Pathways for proton reduction by cobalt complexes are shown in Scheme 1.1. Reduction to a $\mathrm{Co}^{\mathrm{I}}$ species that can be protonated to form $\mathrm{Co}^{\mathrm{III}} \mathrm{H}$ must occur first. The hydride can react in a bimolecular step with another hydride to eliminate $\mathrm{H}_{2}$ (homolytic or bimetallic route, red pathway), or it can be protonated, ${ }^{88}$ release $\mathrm{H}_{2}$, and generate $\mathrm{Co}^{\mathrm{III}}$ that is subsequently reduced (heterolytic or monometallic route, blue pathway). Alternatively, the $\mathrm{Co}^{\mathrm{III}} \mathrm{H}$ can be reduced further to yield $\mathrm{Co}^{\mathrm{II}} \mathrm{H},{ }^{89}$ which can react via an analogous homolytic (orange) or heterolytic (purple) pathway. If the $\mathrm{Co}^{\mathrm{I}}$ species is not protonated, it can be reduced further to a "Co" species (which could be $\mathrm{Co}^{\mathrm{I}}$ with a ligand radical) which, upon protonation to form $\mathrm{Co}^{\mathrm{II}} \mathrm{H}$ (green pathway), can react as above. 


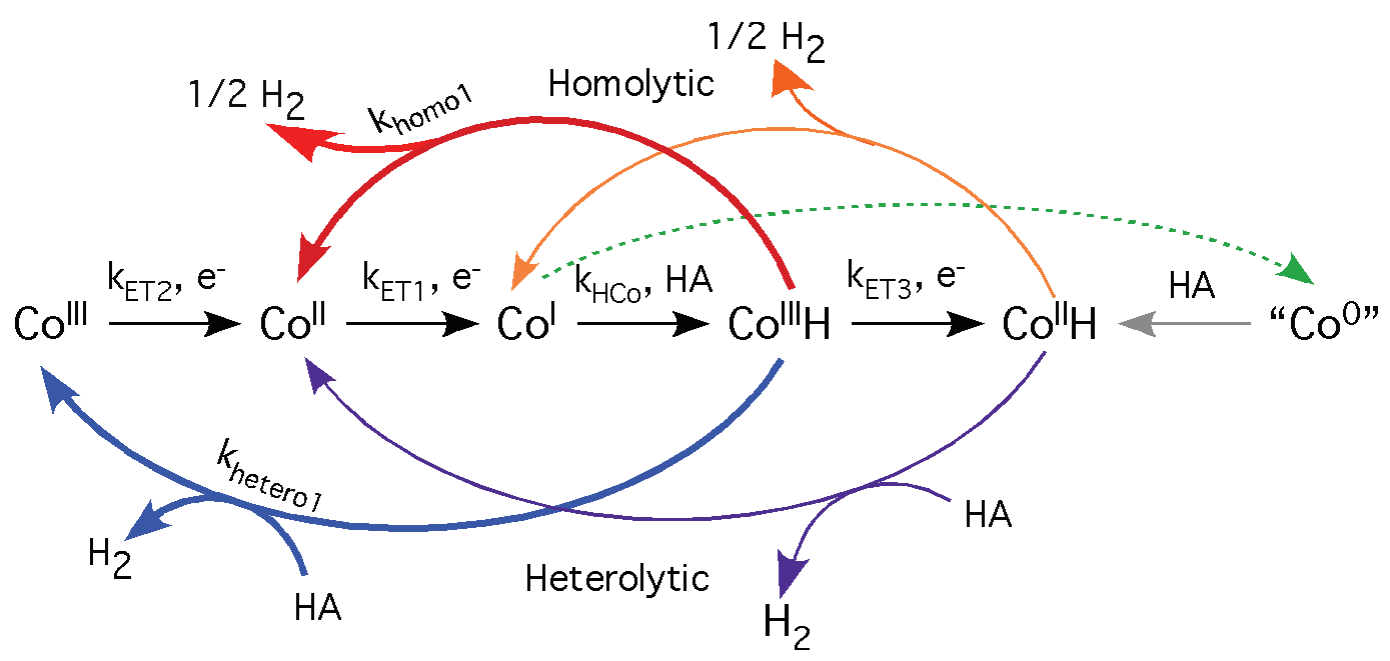

Scheme $1.1 \mathrm{H}_{2}$ evolution pathways.

Chao and Espenson studied the reactivity of kinetically stabilized $\left[\mathrm{HCo}(\mathrm{dmgH})_{2} \mathrm{P}(n-\right.$ $\left.\left.\mathrm{C}_{4} \mathrm{H}_{9}\right)_{3}\right](\mathbf{7 H})$ in order to ascertain the pathway of $\mathrm{H}_{2}$ formation. ${ }^{90}$ Interpretation of hydride reaction kinetics in the presence of acid (aqueous) required a rate expression that was both first and second order in $\mathbf{7 H}$ concentration, suggesting parallel homolytic and heterolytic hydridocobaloxime pathways. Detailed mechanistic work indicated that the homolytic pathway predominates with a rate constant of $1.7 \times 10^{4} \mathrm{M}^{-1} \mathrm{~s}^{-1}$. Since the hydridocobaloxime protonation rate constant was found to be $0.42 \mathrm{M}^{-1} \mathrm{~s}^{-1}$, the heterolytic pathway is competitive only at low catalyst or high acid concentrations.

Fontecave and coworkers investigated catalysis by $\mathbf{1}$ in both DMF and acetonitrile; ${ }^{68}(p-$ cyanoanilinium $) \mathrm{BF}_{4}$ was shown to give rise to irreversible cathodic waves near the $\mathrm{Co}^{\mathrm{I} / \mathrm{I}}$ potential, indicative of electrocatalytic proton reduction. Lower current densities were seen near the same potential when $\mathrm{CF}_{3} \mathrm{COOH}$ was employed as a proton source and an additional catalytic wave appeared near $-1.0 \mathrm{~V}$ vs. SCE, which was attributed to the reduction of $\mathrm{Co}^{\mathrm{III}} \mathrm{H}$ to $\mathrm{Co}^{\mathrm{II}} \mathrm{H}$ and subsequent reaction. With a weak acid, $\left[\mathrm{Et}_{3} \mathrm{NH}\right] \mathrm{Cl}$, catalytic waves were observed near the $\mathrm{Co}^{\mathrm{I} /{ }^{\prime \prime}}$ potential $\left(-1.47 \mathrm{~V}\right.$ vs. SCE). Since $\mathrm{Co}^{\mathrm{I}}$ is not 
sufficiently basic to be protonated by this acid and the thermodynamic potential for hydrogen evolution from $\left[\mathrm{Et}_{3} \mathrm{NH}\right] \mathrm{Cl}$ lies negative of the $\mathrm{Co}^{\mathrm{II} / \mathrm{I}}$ potential, catalysis was not seen until the second reduction. It is noted that the peak currents at these negative potentials were convoluted with direct $\left[\mathrm{Et}_{3} \mathrm{NH}\right] \mathrm{Cl}$ reduction at the glassy carbon electrode. Modeling of the cyclic voltammetry data ${ }^{91}$ suggested that reactions with $(p-$ cyanoanilinium) $\mathrm{BF}_{4}$ and $\mathrm{CF}_{3} \mathrm{COOH}$ proceed by a heterolytic proton reduction (monometallic pathway) via both $\mathrm{Co}^{\mathrm{III}} \mathrm{H}$ and $\mathrm{Co}^{\mathrm{II}} \mathrm{H}$. It was concluded that with acids strong enough to protonate both $\mathrm{Co}^{\mathrm{I}}$ and $\mathrm{Co}^{\mathrm{III}} \mathrm{H}$ (e.g., ( $p$-cyanoanilinium) $\left.\mathrm{BF}_{4}\right)$, hydrogen evolution occurred via a heterolytic pathway. In cases where the acid is strong enough to protonate $\mathrm{Co}^{\mathrm{I}}$, but not the $\mathrm{Co}^{\mathrm{III}} \mathrm{H}$ intermediate, the hydride is further reduced to $\mathrm{Co}^{\mathrm{II}} \mathrm{H}$ before reacting either heterolytically or homolytically. Weak acids like $\left[\mathrm{Et}_{3} \mathrm{NH}\right] \mathrm{Cl}$ that are unable to protonate the $\mathrm{Co}^{\mathrm{I}}$ intermediate can protonate " $\mathrm{Co}^{0}$," and $\mathrm{H}_{2}$ evolution can be catalyzed through the intermediacy of a $\mathrm{Co}^{\mathrm{II}} \mathrm{H}$ species.

Cyclic voltammograms of $\mathbf{1}$ and $\mathbf{2}$ also showed catalytic waves upon addition of TsOH $\bullet \mathrm{H}_{2} \mathrm{O}$, as discussed above. ${ }^{33}$ The shape of the catalytic wave of $\mathbf{1}$ indicated a rapid reaction limited by proton diffusion to the electrode surface. At higher concentrations of $\mathrm{TsOH} \cdot \mathrm{H}_{2} \mathrm{O}$, the wave approached but did not reach a plateau. A quasi-reversible peak at about $-1.0 \mathrm{~V}$ vs. SCE observed for $\mathbf{1}$ at low acid concentration was attributed to the $\mathrm{Co}{ }^{\mathrm{III}} \mathrm{H} / \mathrm{Co}^{\mathrm{II}} \mathrm{H}$ couple; the reduction potential was similar to that seen for cobalt(III) alkyl species. ${ }^{92}$ As catalysis at the $\mathrm{Co}^{\mathrm{II} / \mathrm{I}}$ potential was relatively rapid, the concentration of $\mathrm{Co}^{\mathrm{III}} \mathrm{H}$ remained low in the reaction layer near the electrode, and only small amounts of $\mathrm{Co}^{\mathrm{II}} \mathrm{H}$ were generated under these conditions (the hydride reacts slowly with itself or with acid at low concentrations). 
Catalytic waves for 2 reached a plateau at high concentrations of $\mathrm{TsOH} \bullet \mathrm{H}_{2} \mathrm{O}$, indicating that catalyst reduction at the electrode is equal to the rate of reoxidation. At lower acid concentrations, a second wave observed at $-0.85 \mathrm{~V}$ vs. SCE indicated electrocatalytic hydrogen evolution by a $\mathrm{Co}^{\mathrm{II}} \mathrm{H}$ species formed by reduction of $\mathrm{Co}^{\mathrm{III}} \mathrm{H}$. As the catalytic reaction of $\mathbf{2}$ is slow compared to $\mathbf{1}$, concentrations of $\mathrm{Co}^{\mathrm{III}} \mathrm{H}$ and acid are high enough to yield catalytic behavior upon reduction to $\mathrm{Co}^{\mathrm{II}} \mathrm{H}$.

On the basis of digital simulations of catalytic waves of $\mathbf{1}$ in the presence of $\mathrm{TsOH} \cdot \mathrm{H}_{2} \mathrm{O}$, it was concluded that bimolecular reactivity of $\mathrm{Co}^{\mathrm{III}} \mathrm{H}$ was responsible for hydrogen evolution, consistent with the findings of Chao and Espenson. ${ }^{90}$ Catalytic waves of 2, however, could be simulated equally well assuming either a heterolytic or homolytic mechanism; thus neither reaction pathway has been identified as predominant. Because electrocatalysis occurred near the $\mathrm{Co}^{\mathrm{II} / \mathrm{I}}$ reduction potentials, it was concluded that pathways through $\mathrm{Co}^{\mathrm{II}} \mathrm{H}$ were unlikely, although those routes could open up at more negative potentials.

In an attempt to shed more light on $\mathrm{H}_{2}$ evolution pathways, Peters and coworkers evaluated catalysis by 1 with ( $p$-cyanoanilinium) $\mathrm{BF}_{4}$, employing higher acid:catalyst ratios $(>10)$ than in earlier work. ${ }^{33}$ With ratios less than 10 , these simulations were successful for both homolytic and heterolytic pathways. Over a wider range (1:1 to 40:1), however, the data could only be simulated successfully assuming a homolytic route for $\mathrm{H}_{2}$ evolution.

The overall rate constant for $\mathrm{H}_{2}$ evolution was estimated directly from the plateau currents. For complexes 1 and 2 , the rate constants are $7 \times 10^{3} \mathrm{M}^{-1} \mathrm{~s}^{-1}$ and $\sim 2 \times 10^{2} \mathrm{M}^{-1} \mathrm{~s}^{-1}$, respectively. In both cases, the reactions are first order in acid concentration. On the basis of these data, it is clear that hydride formation is a key step in the catalysis of $\mathrm{H}_{2}$ evolution. 
Attempts to isolate a $\mathrm{Co}^{\mathrm{III}} \mathrm{H}$ complex with glyoxime ligands have been unsuccessful, except for the kinetically stabilized phosphine supported hydride $\mathbf{7 H} .{ }^{90}$ Protonation of isolable $\mathrm{Co}^{\mathrm{I}}$ species with a variety of proton sources, as well as reaction of $\mathrm{Co}^{\mathrm{III}}$ halide species with borohydrides, consistently yielded $\mathrm{Co}^{\mathrm{II}}$ complexes and $\mathrm{H}_{2}$, suggesting that $\mathrm{Co}^{\mathrm{III}} \mathrm{H}$ is extremely reactive. ${ }^{33}$

Photocatalytic hydrogen generation systems are considerably more complex than corresponding electrocatalytic ones. Reactions in photosystems with tertiary amine sacrificial donors (TEOA or $\mathrm{Et}_{3} \mathrm{~N}$ ) are set out in Scheme 1.2. The excited photosensitizer $\left(\mathrm{PS}^{*}\right)$ can be quenched by cobaloxime, producing $\mathrm{PS}^{+}$, which reacts with $\mathrm{R}_{2} \mathrm{~N}-\mathrm{CH}_{2}-\mathrm{R}^{\prime}$ to generate $\mathrm{R}_{2} \mathrm{~N}-\mathrm{CH}_{2}-\mathrm{R}^{\prime+}$; PS ${ }^{*}$ also can be reductively quenched by $\mathrm{R}_{2} \mathrm{~N}-\mathrm{CH}_{2}-\mathrm{R}^{\prime}$, producing $\mathrm{PS}^{-}$. Decomposition of $\mathrm{R}_{2} \mathrm{~N}-\mathrm{CH}_{2}-\mathrm{R}^{\prime+}$ yields a proton and a second reducing equivalent, $\mathrm{R}_{2} \mathrm{~N}-\mathrm{CH}-\mathrm{R}^{\prime}$. Co ${ }^{\mathrm{II}}$ can be reduced by $\mathrm{PS}^{*}, \mathrm{PS}^{-}$, or $\mathrm{R}_{2} \mathrm{~N}-\mathrm{CH}-\mathrm{R}^{\prime}$, and is then protonated to give $\mathrm{Co}^{\mathrm{III}} \mathrm{H}$, which can be reduced to $\mathrm{Co}^{\mathrm{II}} \mathrm{H}$ by any of the powerful reductants. The weakly basic conditions under which these photocatalytic systems operate disfavor protonation of $\mathrm{Co}^{\mathrm{III}} \mathrm{H}$. 


$$
\begin{aligned}
& \mathrm{PS} \stackrel{h v}{\rightarrow} \mathrm{PS}^{*} \\
& \mathrm{PS}^{*}+\mathrm{Co}^{\mathrm{II}} \rightarrow \mathrm{PS}^{+}+\mathrm{Co}^{\mathrm{I}} \\
& \mathrm{PS}^{+}+\mathrm{R}_{2} \ddot{\mathrm{N}}-\mathrm{CH}_{2}-\mathrm{R}^{\prime} \rightarrow \mathrm{PS}+\mathrm{R}_{2} \ddot{\mathrm{N}}-\mathrm{CH}_{2}-\mathrm{R}^{\prime+} \\
& \mathrm{PS}^{*}+\mathrm{R}_{2} \ddot{\mathrm{N}}-\mathrm{CH}_{2}-\mathrm{R}^{\prime} \rightarrow \mathrm{PS}^{-}+\mathrm{R}_{2} \ddot{\mathrm{N}}-\mathrm{CH}_{2}-\mathrm{R}^{\prime+} \\
& \mathrm{PS}^{-}+\mathrm{Co}^{\mathrm{II}} \rightarrow \mathrm{PS}+\mathrm{Co}^{\mathrm{I}} \\
& \mathrm{R}_{2} \ddot{\mathrm{N}}-\mathrm{CH}_{2}-\mathrm{R}^{\prime+} \rightarrow \mathrm{H}^{+}+\mathrm{R}_{2} \ddot{\mathrm{N}}-\mathrm{CH}-\mathrm{R}^{\prime} \\
& \mathrm{R}_{2} \ddot{\mathrm{N}}-\mathrm{CH}-\mathrm{R}^{\prime}+\mathrm{Co}^{\mathrm{II}} \rightarrow \mathrm{R}_{2} \mathrm{~N}^{+}=\mathrm{CH}-\mathrm{R}^{\prime}+\mathrm{Co}^{\mathrm{I}} \\
& \mathrm{Co}^{\mathrm{I}}+\mathrm{H}^{+} \rightarrow \mathrm{Co}^{\mathrm{II}} \mathrm{H} \\
& \mathrm{PS}^{-}+\mathrm{Co}{ }^{\mathrm{III}} \mathrm{H} \rightarrow \mathrm{PS}^{\mathrm{C}}+\mathrm{Co}^{\mathrm{II}} \mathrm{H} \\
& \mathrm{PS}^{*}+\mathrm{Co}{ }^{\mathrm{III}} \mathrm{H} \rightarrow \mathrm{PS}^{+}+\mathrm{Co}^{\mathrm{II}} \mathrm{H} \\
& \mathrm{R}_{2} \ddot{\mathrm{N}}-\mathrm{CH}-\mathrm{R}^{\prime}+\mathrm{Co}^{\mathrm{III}} \mathrm{H} \rightarrow \mathrm{R}_{2} \mathrm{~N}^{+}=\mathrm{CH}-\mathrm{R}^{\prime}+\mathrm{Co}{ }^{\mathrm{II}} \mathrm{H} \\
& \mathrm{R}_{2} \mathrm{~N}^{+}=\mathrm{CH}-\mathrm{R}^{\prime} \rightarrow \text { glycoaldehyde and di(ethanol)amine }
\end{aligned}
$$

Scheme 1.2 $\mathrm{H}_{2}$ evolution pathways in photochemical systems. The $\mathrm{Co}^{\mathrm{III}} \mathrm{H}$ and $\mathrm{Co}^{\mathrm{II}} \mathrm{H}$ intermediates may react via homolytic and heterolytic reaction pathways (Scheme 1.1) to evolve $\mathrm{H}_{2}$. Similar pathways exist with $\mathrm{Et}_{3} \mathrm{~N}$ as a sacrificial electron donor.

Eisenberg's work on photochemical systems using $\mathrm{Pt}^{\mathrm{II}}$ acetylide chromophores ${ }^{69,79}$ and organic photosensitizers ${ }^{83-84}$ with $\mathbf{3}$ (as well as other cobaloximes) showed that hydrogen evolution was first order in catalyst concentration. The authors favor a monometallic route via $\mathrm{Co}^{\mathrm{II}} \mathrm{H}$ to produce hydrogen. The $\left[\mathrm{Re}(\mathrm{CO})_{3} \mathrm{Br}(\right.$ phen $\left.)\right] / \mathbf{1} / \mathrm{Et}_{3} \mathrm{~N} / \mathrm{Et}_{3} \mathrm{NH}^{+}$system examined by Fontecave and coworkers also exhibited a first-order dependence on catalyst concentration, although above a certain concentration the yield decreases, likely owing to competition for light absorption between the colored catalyst and photosensitizer. ${ }^{80}$ In the $\left[\mathrm{Re}(\mathrm{CO}){ }_{3} \mathrm{Br}(\mathrm{bpy})\right] / 4 / \mathrm{TEOA} / \mathrm{AcOH} / \mathrm{DMF}$ system studied by Alberto and coworkers, the observation of a second-order dependence on 4 in the rate of $\mathrm{H}_{2}$ evolution $\left(3.7 \mathrm{M}^{-1} \mathrm{~s}^{-1}\right)$ led to the conclusion that the reaction occurred primarily via a homolytic route, although other mechanisms were not ruled out. ${ }^{81}$ The authors noted that a heterolytic process likely would require the generation of $\mathrm{Co}^{\mathrm{II}} \mathrm{H}$ as a reactive intermediate. 
Lastly, while few investigations have focused on hydrogen evolution in purely aqueous systems, Szajna-Fuller and Bakac utilized spectrophotometric monitoring to determine the kinetics details of hydrogen evolution from solutions containing $\mathrm{Ti}^{\mathrm{III}}$ citrate and $1 .{ }^{93}$ At near neutral $\mathrm{pH}$, protonation of $\mathrm{Co}^{\mathrm{I}}$ was deemed to be fast on the timescale of the overall reaction, and citrate was suspected to be involved as either a proton donor or a ligand for $\mathrm{Co}^{\mathrm{I}}$. Upon protonation, an intermediate, attributed to a citrate coordinated $\mathrm{Co}^{\mathrm{III}} \mathrm{H}$, forms with a maximum absorbance at $770 \mathrm{~nm}$ and disappears rapidly at low $\mathrm{pH}$. Decomposition of the catalyst under the reaction conditions was attributed to the decay of $\left[\mathrm{Co}^{\mathrm{III}}\left(\mathrm{dmgBF}_{2}\right)\right]^{+}$ intermediate. The authors note that these two observations suggest a heterolytic route via $\mathrm{Co}^{\mathrm{III}} \mathrm{H}$ is primarily responsible for $\mathrm{H}_{2}$ evolution.

\section{Prospects for Cobaloxime Chemistry}

Electrochemical and photochemical investigations have shed light on potential pathways of cobalt-catalyzed hydrogen evolution from solutions. To date, however, no studies have provided detailed chemical kinetics of $\mathrm{H}_{2}$ production nor have any key intermediates been detected.

Photochemical methods coupled with time-resolved spectroscopy offer unique opportunities to identify and monitor intermediates in the reaction cycle leading to $\mathrm{H}_{2}$ evolution. For example, laser flash-quench method $s^{94-96}$ can be used to trigger the reduction of $\mathrm{Co}^{\mathrm{II}}$ to $\mathrm{Co}^{\mathrm{I}}$, permitting optical ${ }^{97}$ or infrared ${ }^{98-99}$ detection of intermediates on timescales appreciably shorter than those accessible by conventional stopped-flow spectroscopy. Transient spectroscopic and kinetics measurements can offer critical insights into ratelimiting processes and the mechanism as a whole. A major challenge in this area is the development of photosensitizers and electron relays that are not inhibited by acids. ${ }^{100}$ In this thesis, Chapter 2 and Chapter 3 describe phototriggering methods coupled to time- 
resolved spectroscopic monitoring utilized to obtain kinetics details about the $\mathrm{H}_{2}$ evolution pathways in acetonitrile solutions. Chapter 4 details photoionization and pulseradiolysis methods employed to study the hydrogen evolution pathway in aqueous solutions.

Electrochemical simulations and the thermodynamic and kinetics analysis detailed in Chapter 2 suggest that the homolytic reaction mechanism is a low-barrier pathway for $\mathrm{H}_{2}$ evolution. This route relies on the diffusion of two hydride species together in solution. A rate law with a first-order dependence on both cobalt and acid is fully consistent with electrochemical data and a first-order dependence on cobalt concentration also has been found for photochemical systems. These findings, coupled with the thermodynamic preference for a homolytic route, suggest that the limiting rate may be associated with formation of the hydride. While a bimolecular reaction of cobalt hydrides may not be rate limiting, a homolytic pathway would preclude the immobilization of catalyst on an electrode surface by preventing bimolecular diffusion pathways. Although there has been very little work on immobilizing these cobaloxime species on surfaces, ${ }^{57,101}$ an efficient water splitting device ${ }^{3}$ may require catalysts to be tethered to a photoelectrode surface. ${ }^{43}$

Ultimately, eliminating bimolecular diffusion by covalent linking of cobalt centers in a binuclear catalyst could potentially enhance the rate of $\mathrm{H}_{2}$ production under certain conditions, such as low catalyst concentrations or when catalysts are immobilized. Any increase in rates of $\mathrm{H}_{2}$ production relative to that of a mononuclear analogue could be probed via electrochemical methods. Work on $\mathrm{Ru}$ and Os diporphyrins indicates that cofacial orientation of metallocenters enhances $\mathrm{H}_{2}$ evolution under certain conditions. ${ }^{102-103}$ There have been very few reports of binuclear cobalt species with glyoxime ${ }^{104}$ or glyoximelike ligands, ${ }^{105}$ and none has demonstrated proton reduction activity. Chapter 5 describes 
new binuclear cobaloxime catalysts and their electrocatalytic production of hydrogen, while Chapter 6 introduces bifunctional ligands intended for covalent linkage to silicon surfaces and discusses early surface chemistry reactions aimed at interfacing the individual components of a water splitting device.

\section{References}

1. Lewis, N. S.; Nocera, D. G. Proc. Natl. Acad. Sci. U. S. A. 2006, 103, 15729.

2. Lewis, N. S. MRS Bull. 2007, 32, 808.

3. Gray, H. B. Nat. Chem. 2009, 1, 7.

4. Dempsey, J. L.; Esswein, A. J.; Manke, D. R.; Rosenthal, J.; Soper, J. D.; Nocera, D. G. Inorg. Chem. 2005, 44, 6879.

5. CRC Handbook of Chemistry and Physics; 64 ed.; CRC Press: Boca Raton, Fl, 1983.

6. Koelle, U. New J. Chem. 1992, 16, 157.

7. Eisenberg, R.; Gray, H. B. Inorg. Chem. 2008, 47, 1697.

8. Artero, V.; Fontecave, M. Coord. Chem. Rev. 2005, 249, 1518.

9. Khaselev, O.; Turner, J. A. Science 1998, 280, 425.

10. Dominey, R. N.; Lewis, N. S.; Bruce, J. A.; Bookbinder, D. C.; Wrighton, M. S. J. Am. Chem. Soc. 1982, 104, 467.

11. Kayes, B. M.; Atwater, H. A.; Lewis, N. S. J. Appl. Phys. 2005, 97, 114302.

12. Boettcher, S. W.; Spurgeon, J. M.; Putnam, M. C.; Warren, E. L.; Turner-Evans, D. B.; Kelzenberg, M. D.; Maiolo, J. R.; Atwater, H. A.; Lewis, N. S. Science 2010, 327, 185.

13. Kelzenberg, M. D.; Boettcher, S. W.; Petykiewicz, J. A.; Turner-Evans, D. B.; Putnam, M. C.; Warren, E. L.; Spurgeon, J. M.; Briggs, R. M.; Lewis, N. S.; Atwater, H. A. Nat. Mater. 2010, 9, 239.

14. Britton, H. T. S. In Hydrogen Ions; Chapman \& Hall: London, 1955; Vol. 1, p 55. 
15. Koper, M.; Bouwman, E. Angew. Chem., Int. Ed. 2010, 49, 3723.

16. Frey, M. Chembiochem 2002, 3, 153.

17. Adams, M. W. W. Biochim. Biophys. Acta 1990, 1020, 115.

18. Tard, C. d.; Pickett, C. J. Chem. Rev. 2009, 109, 2245.

19. Gloaguen, F.; Rauchfuss, T. B. Chem. Soc. Rev. 2009, 38, 100.

20. Dempsey, J. L.; Brunschwig, B. S.; Winkler, J. R.; Gray, H. B. Acc. Chem. Res. 2009, 42, 1995.

21. Kellett, R. M.; Spiro, T. G. Inorg. Chem. 1985, 24, 2373.

22. Collin, J. P.; Jouaiti, A.; Sauvage, J. P. Inorg. Chem. 1988, 27, 1986.

23. Efros, L. L.; Thorp, H. H.; Brudvig, G. W.; Crabtree, R. H. Inorg. Chem. 1992, 31, 1722.

24. Krishnan, C. V.; Sutin, N. J. Am. Chem. Soc. 1981, 103, 2141.

25. Rillema, D. P.; Endicott, J. F. Inorg. Chem. 1976, 15, 1459.

26. Abdel-Hamid, R.; El-Sagher, H. M.; Abdel-Mawgoud, A. M.; Nafady, A. Polyhedron 1998, 17, 4535 .

27. Bernhardt, P. V.; Jones, L. A. Inorg. Chem. 1999, 38, 5086.

28. Felton, G. A. N.; Glass, R. S.; Lichtenberger, D. L.; Evans, D. H. Inorg. Chem. 2006, 45, 9181.

29. Himmel, D.; Goll, S. K.; Leito, I.; Krossing, I. Angew. Chem., Int. Ed. 2010, 49, 6885.

30. Fourmond, V.; Jacques, P.-A.; Fontecave, M.; Artero, V. Inorg. Chem. 2010, ASAP.

31. Kaupmees, K.; Kaljurand, I.; Leito, I. J. Phys. Chem. A 2010, 114, 11788.

32. Izutsu, K. Acid-Base Dissociation Constants in Dipolar Aprotic Solvents; Blackwell Scientific Publications: Oxford, U.K., 1990.

33. Hu, X.; Brunschwig, B. S.; Peters, J. C. J. Am. Chem. Soc. 2007, 129, 8988.

34. Darensbourg, M. Y.; Lyon, E. J.; Smee, J. J. Coord. Chem. Rev. 2000, 206, 533. 
35. Wang, Q.; Barclay, J. E.; Blake, A. J.; Davies, E. S.; Evans, D. J.; Marr, A. C.; McInnes, E. J. L.; McMaster, J.; Wilson, C.; Schröder, M. Chem. Eur. J. 2004, 10, 3384.

36. Perra, A.; Davies, E. S.; Hyde, J. R.; Wang, Q.; McMaster, J.; Schroder, M. Chem. Commun. 2006, 1103.

37. Ogo, S.; Kabe, R.; Uehara, K.; Kure, B.; Nishimura, T.; Menon, S. C.; Harada, R.; Fukuzumi, S.; Higuchi, Y.; Ohhara, T.; Tamada, T.; Kuroki, R. Science 2007, 316, 585.

38. Kure, B.; Matsumoto, T.; Ichikawa, K.; Fukuzumi, S.; Higuchi, Y.; Yagi, T.; Ogo, S. Dalton Trans. 2008, 4747.

39. Matsumoto, T.; Kure, B.; Ogo, S. Chem. Lett. 2008, 37, 970.

40. Canaguier, S.; Vaccaro, L.; Artero, V.; Ostermann, R.; Pécaut, J.; Field, M.; Fontecave, M. Chem. Eur. J. 2009, 15, 9350.

41. Oudart, Y.; Artero, V.; Norel, L.; Train, C.; Pécaut, J.; Fontecave, M. J. Organomet. Chem. 2009, 694, 2866.

42. Oudart, Y.; Artero, V.; Pécaut, J.; Fontecave, M. Inorg. Chem. 2006, 45, 4334.

43. Tran, P. D.; Artero, V.; Fontecave, M. Energy Environ. Sci. 2010, 3, 727.

44. Barton, B. E.; Whaley, C. M.; Rauchfuss, T. B.; Gray, D. L. J. Am. Chem. Soc. 2009, 131, 6942.

45. Canaguier, S.; Field, M.; Oudart, Y.; Pecaut, J.; Fontecave, M.; Artero, V. Chem. Commun. 2010, 46, 5876.

46. Liu, X. M.; Ibrahim, S. K.; Tard, C.; Pickett, C. J. Coord. Chem. Rev. 2005, 249, 1641.

47. Capon, J. F.; Gloaguen, F.; Schollhammer, P.; Talarmin, J. Coord. Chem. Rev. 2005, 249, 1664.

48. Gloaguen, F.; Lawrence, J. D.; Rauchfuss, T. B. J. Am. Chem. Soc. 2001, 123, 9476.

49. Capon, J.-F.; Gloaguen, F.; Pétillon, F. Y.; Schollhammer, P.; Talarmin, J. Coord. Chem. Rev. 2009, 253, 1476. 
50. Wilson, A. D.; Newell, R. H.; McNevin, M. J.; Muckerman, J. T.; Rakowski DuBois, M.; DuBois, D. L. J. Am. Chem. Soc. 2006, 128, 358.

51. Fraze, K.; Wilson, A. D.; Appel, A. M.; Rakowski DuBois, M.; DuBois, D. L. Organometallics 2007, 26, 3918.

52. Wilson, A. D.; Shoemaker, R. K.; Miedaner, A.; Muckerman, J. T.; DuBois, D. L.; DuBois, M. R. Proc. Natl. Acad. Sci. U. S. A. 2007, 104, 6951.

53. Yang, J. Y.; Bullock, R. M.; Shaw, W. J.; Twamley, B.; Fraze, K.; DuBois, M. R.; DuBois, D. L. J. Am. Chem. Soc. 2009, 131, 5935.

54. DuBois, M. R.; DuBois, D. L. Chem. Soc. Rev. 2009, 38, 62.

55. Appel, A. M.; DuBois, D. L.; Rakowski DuBois, M. J. Am. Chem. Soc. 2005, 127, 12717.

56. Jacques, P.-A.; Artero, V.; Pécaut, J.; Fontecave, M. Proc. Nat. Acad. Sci. U.S.A. 2009, $106,20627$.

57. Berben, L. A.; Peters, J. C. Chem. Commun. 2010, 46, 398.

58. Losse, S.; Vos, J. G.; Rau, S. Coord. Chem. Rev. 2010, 254, 2492.

59. Jacobsen, G. M.; Yang, J. Y.; Twamley, B.; Wilson, A. D.; Bullock, R. M.; DuBois, M. R.; DuBois, D. L. Energy Environ. Sci. 2008, 1, 167.

60. Bigi, J. P.; Hanna, T. E.; Harman, W. H.; Chang, A.; Chang, C. J. Chem. Commun. 2010, $46,958$.

61. Endicott, J. F.; Rillema, D. P.; Papaconstantinou, E. Inorg. Chem. 1971, 10, 1739.

62. Tait, A. M.; Hoffman, M. Z.; Hayon, E. J. Am. Chem. Soc. 1976, 98, 86.

63. Brown, G. M.; Brunschwig, B. S.; Creutz, C.; Endicott, J. F.; Sutin, N. J. Am. Chem. Soc. $1979,101,1298$.

64. Connolly, P.; Espenson, J. H. Inorg. Chem. 1986, 25, 2684.

65. Karunadasa, H. I.; Chang, C. J.; Long, J. R. Nature 2010, 464, 1329.

66. Tovrog, B. S.; Kitko, D. J.; Drago, R. S. J. Am. Chem. Soc. 1976, 98, 5144. 
67. Hu, X. L.; Cossairt, B. M.; Brunschwig, B. S.; Lewis, N. S.; Peters, J. C. Chem. Commun. 2005, 4723.

68. Baffert, C.; Artero, V.; Fontecave, M. Inorg. Chem. 2007, 46, 1817.

69. Du, P.; Schneider, J.; Luo, G.; Brennessel, W. W.; Eisenberg, R. Inorg. Chem. 2009, 48, 4952.

70. Pavlishchuk, V. V.; Addison, A. W. Inorg. Chim. Acta 2000, 298, 97.

71. Creutz, C.; Chou, M. H.; Fujita, E.; Szalda, D. J. Coord. Chem. Rev. 2005, 249, 375.

72. Razavet, M.; Artero, V.; Fontecave, M. Inorg. Chem. 2005, 44, 4786.

73. Connelly, N. G.; Geiger, W. E. Chem. Rev. 1996, 96, 877.

74. Schrauzer, G. N.; Windgassen, R. J. J. Am. Chem. Soc. 1966, 88, 3738.

75. Gray, H. B.; Maverick, A. W. Science 1981, 214, 1201.

76. Esswein, A. J.; Nocera, D. G. Chem. Rev. 2007, 107, 4022.

77. Hawecker, J.; Lehn, J. M.; Ziessel, R. Nouv. J. Chim. 1983, 7, 271.

78. Wang, M.; Na, Y.; Gorlov, M.; Sun, L. Dalton Trans. 2009, 6458.

79. Du, P.; Knowles, K.; Eisenberg, R. J. Am. Chem. Soc. 2008, 130, 12576.

80. Fihri, A.; Artero, V.; Pereira, A.; Fontecave, M. Dalton Trans. 2008, 5567.

81. Probst, B.; Kolano, C.; Hamm, P.; Alberto, R. Inorg. Chem. 2009, 48, 1836.

82. Probst, B.; Rodenberg, A.; Guttentag, M.; Hamm, P.; Alberto, R. Inorg. Chem. 2010, 49, 6453.

83. Lazarides, T.; McCormick, T.; Du, P.; Luo, G.; Lindley, B.; Eisenberg, R. J. Am. Chem. Soc. 2009, 131, 9192.

84. McCormick, T. M.; Calitree, B. D.; Orchard, A.; Kraut, N. D.; Bright, F. V.; Detty, M. R.; Eisenberg, R. J. Am. Chem. Soc. 2010, 132, 15480.

85. Zhang, P.; Wang, M.; Dong, J. F.; Li, X. Q.; Wang, F.; Wu, L. Z.; Sun, L. C. J. Phys. Chem. C 2010, 114, 15868. 
86. Fihri, A.; Artero, V.; Razavet, M.; Baffert, C.; Leibl, W.; Fontecave, M. Angew. Chem., Int. Ed. 2008, 47, 564.

87. Li, C.; Wang, M.; Pan, J. X.; Zhang, P.; Zhang, R.; Sun, L. C. J. Organomet. Chem. 2009, 694, 2814.

88. Besora, M.; Lledos, A.; Maseras, F. Chem. Soc. Rev. 2009, 38, 957.

89. Bhugun, I.; Lexa, D.; Savéant, J.-M. J. Am. Chem. Soc. 1996, 118, 3982.

90. Chao, T.-H.; Espenson, J. H. J. Am. Chem. Soc. 1978, 100, 129.

91. Rudolf, M. DigiElch 2.0, http://digielch.de/.

92. Shi, S.; Bakac, A.; Espenson, J. H. Inorg. Chem. 1991, 30, 3410.

93. Szajna-Fuller, E.; Bakac, A. Eur. J. Inorg. Chem. 2010, 2010, 2488.

94. Ogata, T.; Yanagida, S.; Brunschwig, B. S.; Fujita, E. J. Am. Chem. Soc. 1995, 117, 6708.

95. Berglund, J.; Pascher, T.; Winkler, J. R.; Gray, H. B. J. Am. Chem. Soc. 1997, 119, 2464.

96. Bjerrum, M. J.; Casimiro, D. R.; Chang, I. J.; Dibilio, A. J.; Gray, H. B.; Hill, M. G.; Langen, R.; Mines, G. A.; Skov, L. K.; Winkler, J. R.; Wuttke, D. S. J. Bioenerg. Biomembr. 1995, 27, 295.

97. Na, Y.; Wang, M.; Pan, J.; Zhang, P.; Åkermark, B.; Sun, L. Inorg. Chem. 2008, 47, 2805.

98. Wright, J. A.; Pickett, C. J. Chem. Commun. 2009, 5719.

99. Shih, C.; Museth, A. K.; Abrahamsson, M.; Blanco-Rodriguez, A. M.; Di Bilio, A. J.; Sudhamsu, J.; Crane, B. R.; Ronayne, K. L.; Towrie, M.; Vlcek, A., Jr.; Richards, J. H.; Winkler, J. R.; Gray, H. B. Science 2008, 320, 1760.

100. Na, Y.; Pan, J.; Wang, M.; Sun, L. Inorg. Chem. 2007, 46, 3813.

101. Pantani, O.; Anxolabehere-Mallart, E.; Aukauloo, A.; Millet, P. Electrochem. Commun. 2007, 9, 54 .

102. Collman, J. P.; Wagenknecht, P. S.; Lewis, N. S. J. Am. Chem. Soc. 1992, 114, 5665. 
103. Collman, J. P.; Ha, Y.; Wagenknecht, P. S.; Lopez, M. A.; Guilard, R. J. Am. Chem. Soc. 1993, 115, 9080.

104. Bhuyan, M.; Laskar, M.; Gupta, B. D. Organometallics 2008, 27, 594.

105. Shimakoshi, H.; Koga, M.; Hisaeda, Y. Bull. Chem. Soc. Jpn. 2002, 75, 1553. 
Chapter 2

PATHWAYS OF $\mathrm{H}_{2}$ EVOLUTION: BARRIERS AND DRIVING FORCES

Portions of this work are excerpted with permission from:

Dempsey, J. L.; Winkler, J. R.; Gray, H. B. J. Am. Chem. Soc. 2010, 132, 1060-1065.

Copyright 2010 American Chemical Society 
Introduction

Difluoroboryl bridged Co ${ }^{\mathrm{II}}$-diglyoxime complexes $\left(\left[\mathrm{Co}\left(\mathrm{dpgBF}_{2}\right)_{2}\left(\mathrm{CH}_{3} \mathrm{CN}\right)_{2}\right] \quad\right.$ (1), $\left[\mathrm{Co}\left(\mathrm{dmgBF}_{2}\right)_{2}\left(\mathrm{CH}_{3} \mathrm{CN}\right)_{2}\right](2)$ : dpg = difluoroboryl-diphenylglyoxime, dmg = difluoroboryldimethylglyoxime $)^{1}$ are among a handful of catalysts ${ }^{2-4}$ that catalyze $\mathrm{H}_{2}$ evolution at relatively high rates with modest overpotentials. To better understand this efficiency, mechanistic details of the catalytic reaction pathway need to be obtained. To date, insight into the mechanism of $\mathrm{H}_{2}$ evolution by these complexes has relied on digital simulations of electrochemical data, ${ }^{5-6}$ not direct measurements of reaction kinetics.

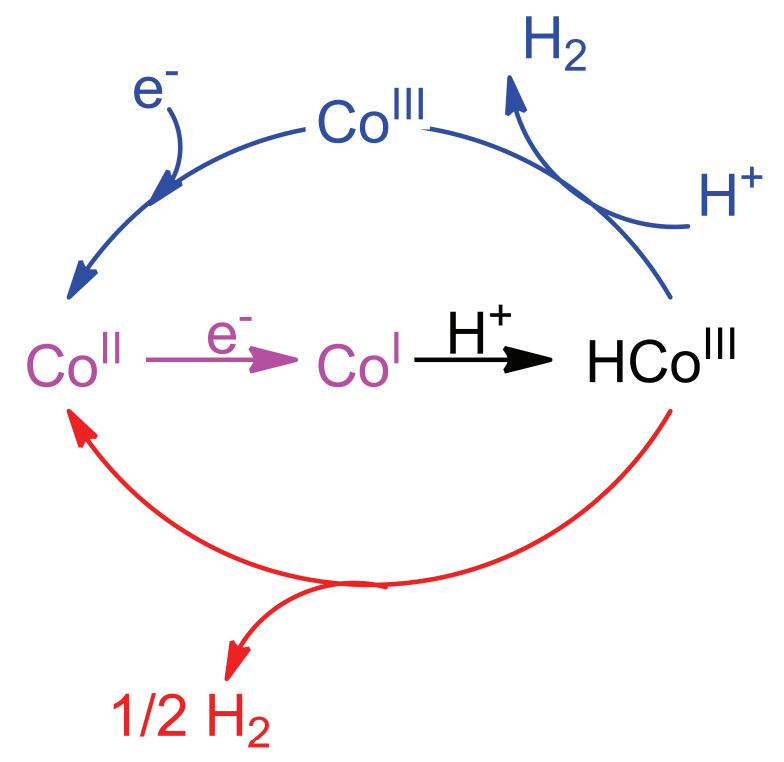

Figure 2.1 Homolytic (red) and heterolytic (blue) reaction pathways for catalysis of $\mathrm{H}_{2}$ evolution by $\mathrm{Co}^{\mathrm{II}}$ complexes. Reducing equivalents (D) deliver electrons to $\mathrm{Co}^{\mathrm{II}}$ complexes and the resulting $\mathrm{Co}^{\mathrm{I}}$ species are protonated to form $\mathrm{Co}^{\mathrm{III}} \mathrm{H}$ intermediates. In the homolytic pathway, two $\mathrm{Co}^{\mathrm{III}} \mathrm{H}$ species undergo bimolecular reductive elimination to produce $\mathrm{H}_{2}$. In the heterolytic pathway, protonation of $\mathrm{Co}^{\mathrm{III}} \mathrm{H}$ is responsible for $\mathrm{H}_{2}$ release, and the $\mathrm{Co}^{\mathrm{III}}$ species is reduced back to $\mathrm{Co}^{\mathrm{II}}$ by $\mathrm{D}$. 
A Co ${ }^{\mathrm{III}}$-hydride $\left(\mathrm{Co}^{\mathrm{III}} \mathrm{H}\right)$ formed in the reaction between a $\mathrm{Co}^{\mathrm{I}}$ complex and a proton donor (HA) likely is a key intermediate in the catalytic cycle. ${ }^{7}$ Production of $\mathrm{H}_{2}$ from $\mathrm{Co}^{\mathrm{III}} \mathrm{H}$ can occur via homolysis (Figure 2.1, red path) or heterolysis (Figure 2.1, blue path) of the $\mathrm{Co}^{\mathrm{III}}-\mathrm{H}$ bond. ${ }^{5-6}$ Efficient catalysis by the heterolytic pathway demands that the $\mathrm{Co}^{\mathrm{III}}$ product of $\mathrm{Co}^{\mathrm{III}}-\mathrm{H}$ bond heterolysis be converted rapidly to $\mathrm{Co}^{\mathrm{II}}$ in the pool of reducing equivalents.

Here, the kinetics and thermodynamics of active $\mathrm{Co}^{\mathrm{II}}$ catalysts have been investigated. A laser flash-quench method $^{8-9}$ has been employed to trigger the reduction of [Có $\left.\mathbf{0}^{\mathrm{II}}\right]$ $\left(\left[\mathrm{Co}\left(\mathrm{dpgBF}_{2}\right)_{2}\left(\mathrm{CH}_{3} \mathrm{CN}\right)_{2}\right], 2\right)$ to $\left[\mathrm{Co}^{\mathrm{I}}\right]\left(\left[\mathrm{Co}\left(\mathrm{dpgBF}_{2}\right)_{2}\left(\mathrm{CH}_{3} \mathrm{CN}\right)\right]^{-}, 2^{-}\right)$, and time-resolved spectroscopy was utilized to identify and monitor the short-lived species. This method permits observation of discrete intermediates on timescales appreciably shorter than those accessible by conventional stopped-flow spectroscopy. Analyses of these flash-quench kinetics data have revealed the barriers for key elementary steps in the catalytic cycle leading to the formation of $\mathrm{H}_{2}$.

\section{Results}

Photochemistry

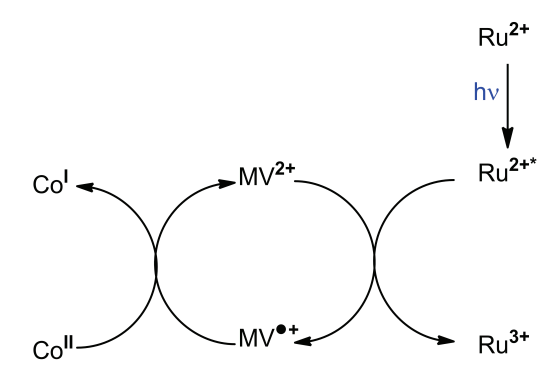

Scheme 2.1 
The photochemical method utilizes methyl viologen $\left(\mathrm{MV}^{2+}\right)$ oxidatively to quench pulsed-laser excited $\left[\mathrm{Ru}(\mathrm{bpy})_{3}\right]^{2+}(\mathrm{bpy}=2,2$ '-bipyridine). The reduced viologen species, $\mathrm{MV}^{\bullet+}$, delivers an electron to $\left[\mathbf{C o}^{\mathrm{II}}\right]$ producing anionic $\left[\mathrm{Co}^{\mathrm{I}}\right]$ and $\left[\mathrm{Ru}(\mathrm{bpy})_{3}\right]^{3+}($ Scheme 2.1, Figure 2.2). The transient absorption (TA) spectrum recorded $250 \mathrm{~ns}$ after excitation is dominated by spectral features characteristic of both $\left[\mathrm{Ru}(\mathrm{bpy})_{3}\right]^{3+}$, bleaching at $450 \mathrm{~nm}$, and $\mathrm{MV}^{\bullet+}$, absorbing at 390 and $605 \mathrm{~nm}$ (Figure 2.3). Between 20 and $100 \mu \mathrm{s}$, the shift in the red absorption feature to $686 \mathrm{~nm}$ with concomitant loss of the $390 \mathrm{~nm}$ absorbance exactly matches the spectroscopic signature of $\left[\mathrm{Co}^{\mathrm{I}}\right]$ (Figure 2.4). During the time interval $500 \mu \mathrm{s}-$ $50 \mathrm{~ms},\left[\mathbf{C o}^{\mathrm{I}}\right]$ reacts with two oxidants in solution, $\left[\mathrm{Ru}(\mathrm{bpy})_{3}\right]^{3+}$ and $\left[\mathrm{Co}^{\mathrm{III}}\right]$ $\left(\left[\mathrm{Co}\left(\mathrm{dpgBF}_{2}\right)_{2}\left(\mathrm{CH}_{3} \mathrm{CN}\right)_{2}\right]^{+}\right)$, the latter produced transiently via $\left[\mathrm{Ru}(\mathrm{bpy})_{3}\right]^{3+}$ oxidation of $\left[\mathrm{Co}^{\mathrm{II}}\right]$.

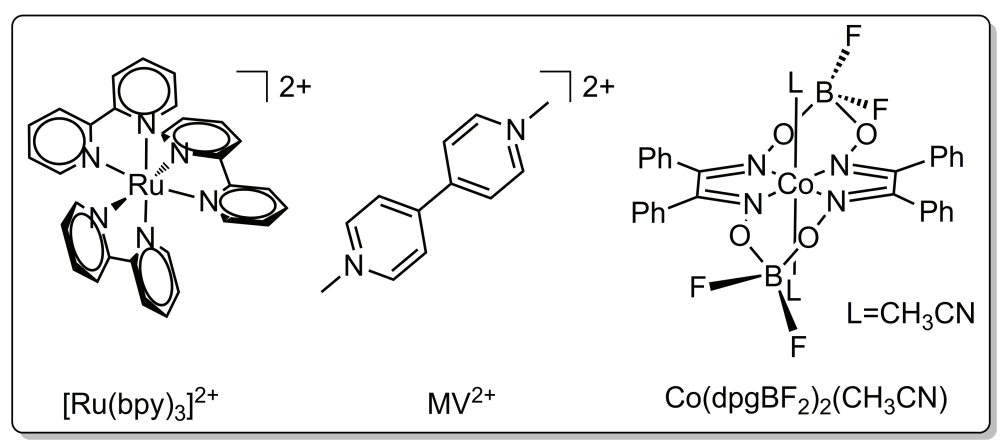

Figure 2.2 Photosensitizer, electron relay, and catalyst employed in this study. 


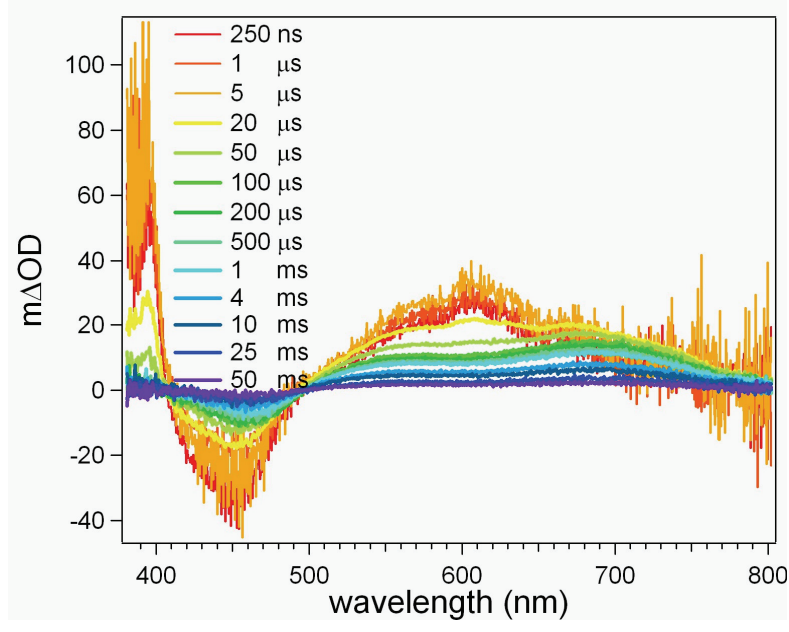

Figure 2.3 Transient difference spectra $20 \mu \mathrm{M}\left[\mathrm{Ru}(\mathrm{bpy})_{3}\right]\left[\mathrm{PF}_{6}\right]_{2}$, $3.5 \mathrm{mM} \quad[\mathrm{MV}]\left[\mathrm{PF}_{6}\right]_{2}, \quad 0.1 \quad \mathrm{M} \quad \mathrm{NBu}_{4} \mathrm{PF}_{6}, \quad$ and $100 \mu \mathrm{M}$ $\mathrm{Co}(\mathrm{dpgBF})_{2}\left(\mathrm{CH}_{3} \mathrm{CN}\right)_{2}$ in $\mathrm{CH}_{3} \mathrm{CN}$ at selected time delays after laser excitation. $\lambda_{\mathrm{ex}}=355 \mathrm{~nm}$.

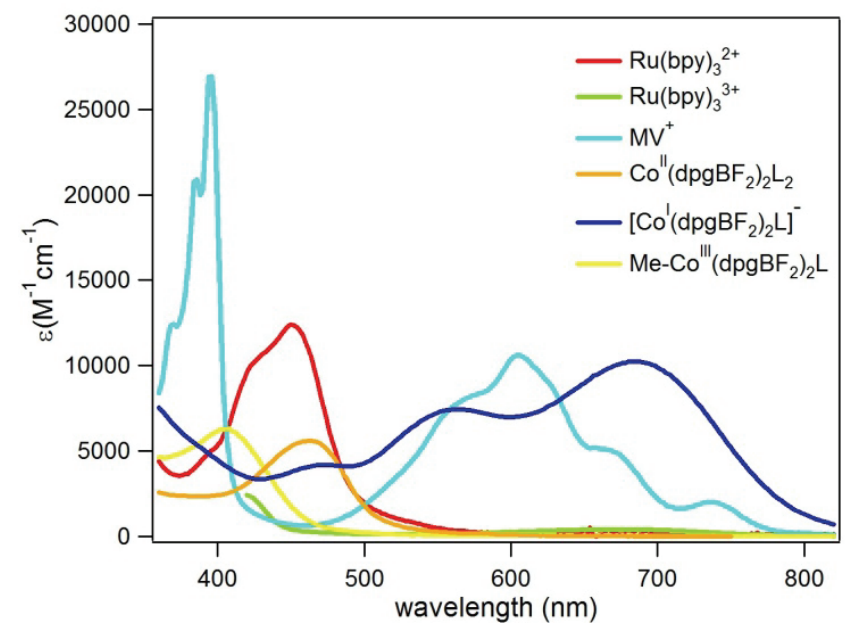

Figure 2.4 Absorption spectra of reactants and intermediates.

The transient difference spectra were fit with linear combinations of the following three molar difference spectra:

$\Delta \varepsilon_{1}(\lambda)=\left[\varepsilon_{R u^{3+}}(\lambda)+\varepsilon_{M V^{*+}}(\lambda)-\varepsilon_{R u^{2+}}(\lambda)-\varepsilon_{M V^{2+}}(\lambda)\right]$ 


$$
\begin{aligned}
& \Delta \varepsilon_{2}(\lambda)=\left[\varepsilon_{R u^{3+}}(\lambda)+\varepsilon_{C o^{+}}(\lambda)-\varepsilon_{R u^{2+}}(\lambda)-\varepsilon_{C o^{2+}}(\lambda)\right] \\
& \Delta \varepsilon_{3}(\lambda)=\left[\varepsilon_{C o^{3+}}(\lambda)+\varepsilon_{C o^{+}}(\lambda)-2 \varepsilon_{\mathrm{Co}^{2+}}(\lambda)\right]
\end{aligned}
$$

where $\Delta \varepsilon_{1}(\lambda), \Delta \varepsilon_{2}(\lambda)$, and $\Delta \varepsilon_{3}(\lambda)$ describe differences in molar extinction coefficients between charge transfer pairs. Vectors $(\Delta \mathbf{A})$ describing the time-resolved difference spectra at discrete wavelengths $(\lambda, 400-800 \mathrm{~nm})$ were generated for several delay times, t. A matrix, $\Delta \varepsilon$, was formulated to describe the molar difference spectra described above, $\Delta \varepsilon=\left[\Delta \varepsilon_{1}(\lambda)\right.$, $\left.\Delta \varepsilon_{2}(\lambda), \Delta \varepsilon_{3}(\lambda)\right]$. An approximation for $\varepsilon_{C o^{3+}}(\lambda)$ was made $\left(\varepsilon_{\mathrm{Co}^{3+}}(\lambda=400-800 \mathrm{~nm})=0\right)$, as $\left[\mathbf{C o}^{\mathrm{III}}\right]$ is not isolable. Note, however, that the absorption profile of the $\mathrm{Co}^{\mathrm{III}} \mathrm{Me}$ species, $\mathrm{Me}$ $\mathrm{Co}\left(\mathrm{dpgBF}_{2}\right)_{2} \mathrm{~L}_{2}$, only has a weak feature at $406 \mathrm{~nm}$ and a tail into the visible region. As the transient difference spectra should be a linear combination of $\Delta \varepsilon_{i}(i=1,2,3)$, the expression $\Delta \mathrm{A}=\Delta \boldsymbol{\varepsilon} \times \boldsymbol{\alpha}$ was solved for $\boldsymbol{\alpha}\left(\boldsymbol{\alpha}=\Delta \boldsymbol{\varepsilon}^{-1} \times \Delta \mathrm{A}\right)$, where $\boldsymbol{\alpha}$ is a column vector reflecting the contributions of the three molar difference spectra to the experimental spectrum. Fits of the transient spectra were generated using the expression $\Delta \mathbf{A}_{\mathrm{fit}}=\Delta \boldsymbol{\varepsilon x \boldsymbol { \alpha }}$. The process was repeated for time delays of $20,50,100,200$, and $500 \mu$ s, and fits overlaid with the difference spectra (Figure 2.5). Small deviations in the fits were observed in the $400-500 \mathrm{~nm}$ region and attributed to the approximation made for $\varepsilon_{\mathrm{Co}^{3+}}(\lambda)$. 


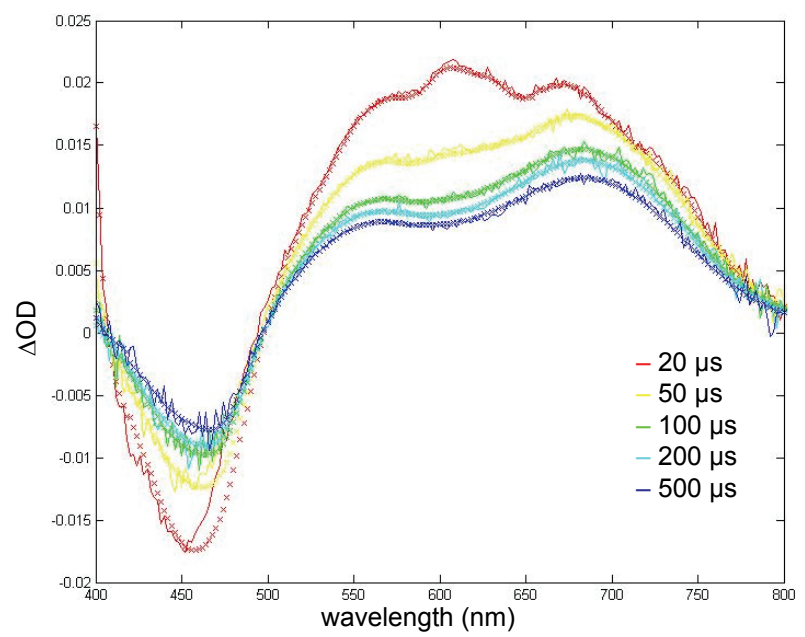

Figure 2.5 Time-resolved transient difference spectra (-) and

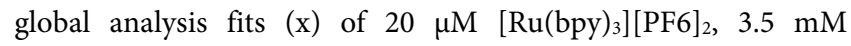
$[\mathrm{MV}]\left[\mathrm{PF}_{6}\right]_{2}, 0.1 \mathrm{M} \mathrm{NBu}_{4} \mathrm{PF}_{6}, 100 \mu \mathrm{M} \mathrm{Co}\left(\mathrm{dpgBF}_{2}\right)_{2}\left(\mathrm{CH}_{3} \mathrm{CN}\right)_{2}$ in $\mathrm{CH}_{3} \mathrm{CN}$ at selected time delays after laser excitation. $\lambda_{\text {ex }}=355 \mathrm{~nm}$. Absorbance $(400-800 \mathrm{~nm}) \mathrm{Co}\left(\mathrm{dpgBF}_{2}\right)_{2}\left(\mathrm{CH}_{3} \mathrm{CN}\right)_{2}$ set equal to 0 .

A kinetics model (Scheme 2.2) was used to describe the photochemical electron transfer (ET) reaction scheme. The decay of the excited state of $\left[\mathrm{Ru}(\mathrm{bpy})_{3}\right]^{2+}$ is described by rate constant $k_{1}$, and measured via time-resolved luminescence $\left(1.04 \times 10^{6} \mathrm{~s}^{-1}\right)$. Bimolecular oxidative quenching by $\mathrm{MV}^{2+}$ is described by $k_{2}$, and the charge recombination by $k_{3}$. SternVolmer quenching was used to determine $k_{2}$; the value was consistent with previous reports (1.58 x $10^{9} \mathrm{M}^{-1} \mathrm{~s}^{-1}$, Figure 2.6). ${ }^{10}$ The single-wavelength transient absorption $\left(\lambda_{\mathrm{ex}}=480 \mathrm{~nm}\right.$, $\left.\lambda_{\text {obs }}=730 \mathrm{~nm}\right)$ of a sample containing $20 \mu \mathrm{M}\left[\mathrm{Ru}(\mathrm{bpy})_{3}\right]\left[\mathrm{PF}_{6}\right]_{2}, 0.1 \mathrm{M} \mathrm{NBu}_{4} \mathrm{PF}_{6}, 3.5 \mathrm{mM}$ $[\mathrm{MV}]\left[\mathrm{PF}_{6}\right]_{2}$, was modeled with second-order kinetics to obtain a value for $k_{3}$ that was consistent with literature reports $\left(5.7 \times 10^{9} \mathrm{M}^{-1} \mathrm{~s}^{-1}\right.$, Figure 2.7$) .{ }^{11}$ 


$$
\begin{array}{ll}
\mathrm{Ru}^{2+*} \rightarrow \mathrm{Ru}^{2+} & k_{1} \\
\mathrm{Ru}^{2+*}+\mathrm{MV}^{2+} \rightarrow \mathrm{Ru}^{3+}+\mathrm{MV}^{\bullet+} & k_{2} \\
\mathrm{Ru}^{3+}+\mathrm{MV}^{\bullet+} \rightarrow \mathrm{Ru}^{2+}+\mathrm{MV}^{2+} & k_{3} \\
\mathrm{MV}^{\circ+}+\mathrm{Co}^{2+} \rightarrow \mathrm{MV}^{2+}+\mathrm{Co}^{+} & k_{4} \\
\mathrm{Ru}^{3+}+\mathrm{Co}^{+} \rightarrow \mathrm{Ru}^{2+}+\mathrm{Co}^{2+} & k_{5} \\
\mathrm{Ru}^{3+}+\mathrm{Co}^{2+} \rightarrow \mathrm{Ru}^{2+}+\mathrm{Co}^{3+} & k_{6} \\
\mathrm{MV}^{++}+\mathrm{Co}^{3+} \rightarrow \mathrm{MV}^{2+}+\mathrm{Co}^{2+} & k_{7} \\
\mathrm{Co}^{3+}+\mathrm{Co}^{+} \rightarrow 2 \mathrm{Co}^{2+} & k_{8}
\end{array}
$$

Scheme 2.2

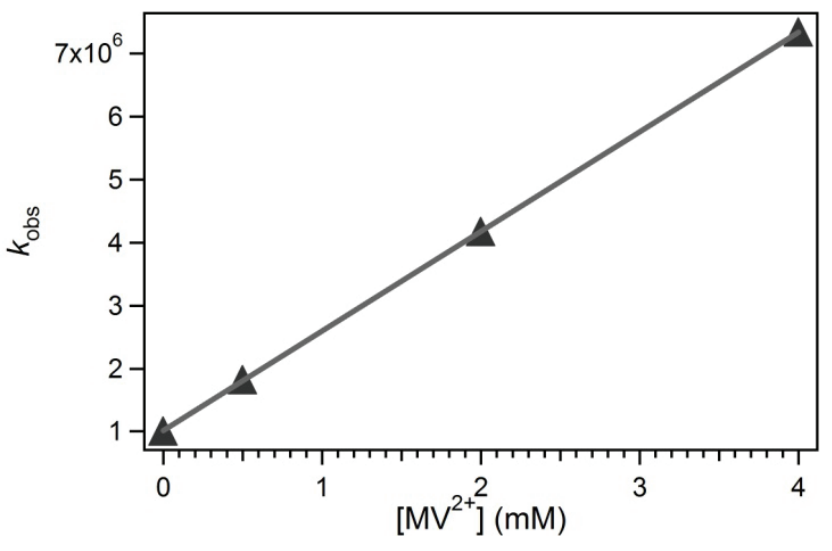

Figure 2.6 Stern-Volmer quenching plot for $\left[\mathrm{Ru}(\mathrm{bpy})_{3}\right]^{2+}$ and $\mathrm{MV}^{2+}$. First-order rate constants obtained from the decay of $\left[\mathrm{Ru}(\mathrm{bpy})_{3}\right]^{2+^{*}}\left(\lambda_{\mathrm{ex}}=480 \mathrm{~nm}, \lambda_{\mathrm{obs}}=630 \mathrm{~nm}\right)$ in the presence of $[\mathrm{MV}]\left[\mathrm{PF}_{6}\right]_{2} . k_{\mathrm{obs}}$ is linearly dependent on the concentration of $\mathrm{MV}^{2+} . k_{0}=k_{0}+k_{\mathrm{q}}\left[\mathrm{MV}^{2+}\right], k_{0}=1.02 \times 10^{6} \mathrm{~s}^{-1}, k_{\mathrm{q}}\left(k_{2}\right)=1.58 \times 10^{9} \mathrm{M}^{-1}$ $\mathrm{s}^{-1}$. Conditions: $21 \mu \mathrm{M}\left[\mathrm{Ru}(\mathrm{bpy})_{3}\right]\left[\mathrm{PF}_{6}\right]_{2}, 0.1 \mathrm{M}\left[{ }^{\mathrm{n}} \mathrm{Bu}_{4} \mathrm{~N}\right]\left[\mathrm{PF}_{6}\right]$, varying concentrations of $[\mathrm{MV}]\left[\mathrm{PF}_{6}\right]_{2}$ in acetonitrile. 


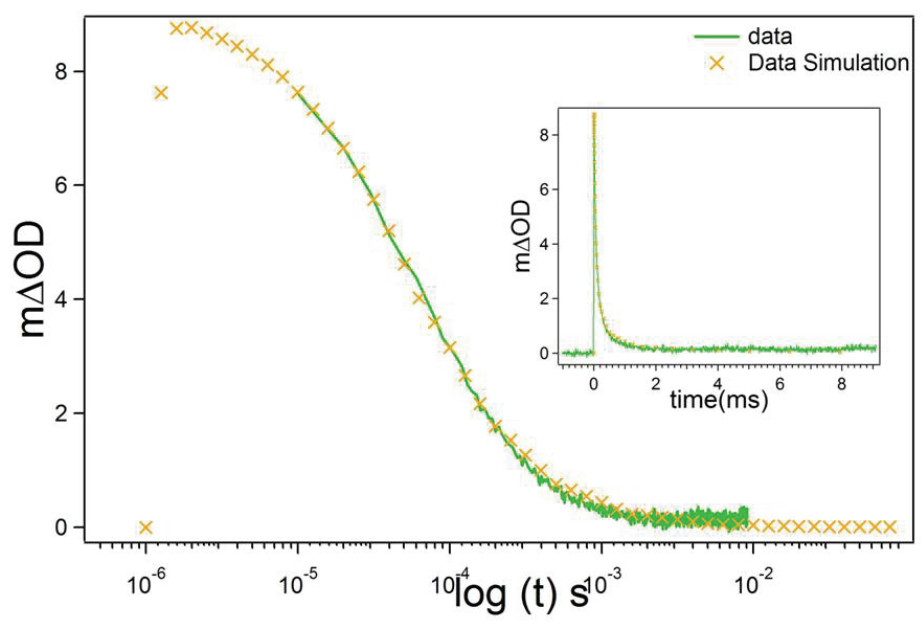

Figure 2.7 Kinetics trace of $20 \mu \mathrm{M}\left[\mathrm{Ru}(\mathrm{bpy})_{3}\right]\left[\mathrm{PF}_{6}\right]_{2}, 3.5 \mathrm{mM}$ $[\mathrm{MV}]\left[\mathrm{PF}_{6}\right]_{2}$, and $0.1 \mathrm{M} \mathrm{NBu} 4 \mathrm{PF}_{6}$ and the simulated fit in logarithmic time. $\lambda_{\text {ex }}=480 \mathrm{~nm}, \lambda_{\text {obs }}=730 \mathrm{~nm}$. ET rate constants used in the simulation: $k_{1}, 1.04 \times 10^{6} \mathrm{~s}^{-1} ; k_{2}, 1.58 \times 10^{9} \mathrm{M}^{-1} \mathrm{~s}^{-1} ; k_{3}$, $5.7 \times 10^{9} \mathrm{M}^{-1} \mathrm{~s}^{-1}$. Inset: kinetics and fit plotted on a linear time axis.

Electron transfer from $\mathrm{MV}^{\bullet+}$ to $\left[\mathrm{Co}^{\mathrm{II}}\right]$ is described by $k_{4} \cdot \mathrm{Ru}(\mathrm{bpy})_{3}{ }^{3+}$ oxidation of $\left[\mathrm{Co}^{\mathrm{I}}\right]$ and [Co $\left.{ }^{\mathrm{II}}\right]$ is identified with rate constants $k_{5}$ and $k_{6}$, respectively. Charge recombination reactions between $\left[\mathbf{C o}^{\mathrm{III}}\right]$ and either $\mathrm{MV}^{\bullet+}$ or $\left[\mathrm{Co}^{\mathrm{I}}\right]$ are described by $k_{7}$ and $k_{8}$, respectively. Kinetics simulations were performed with differential equations (Scheme 2.3) describing the concentrations of all species involved in these ET reactions. Inputs to solve the initial value problem included a vector describing initial concentrations of reactants and products, a time-span vector $\left(10^{-6}\right.$ to $\left.10^{-1} \mathrm{~s}\right)$, and a vector of rate constants $k_{1}-k_{8}$. Profiles of concentration changes versus time were plotted for each individual species. These concentration profiles were translated to simulated transient difference spectra by summing the products of a concentration profile multiplied by an estimated absorption coefficient at $730 \mathrm{~nm}$ for each species (Table 2.1, Appendix C). The rate constants $\left(k_{4}, k_{5}, k_{6}, k_{7}\right.$, and $\left.k_{8}\right)$ were iteratively adjusted until a satisfactory simulation of the TA kinetics was obtained (Figure 2.8). Simulations were repeated for six different sample measurements containing 
variable amounts of $\mathbf{C o}^{\text {II }}(63-157 \mu \mathrm{M})$, and were iterated to obtain a self-consistent set of rate constants $\left(k_{4}, 2.0 \times 10^{8} \mathrm{M}^{-1} \mathrm{~s}^{-1} ; k_{5}, 9.8 \times 10^{9} \mathrm{M}^{-1} \mathrm{~s}^{-1} ; k_{6}, 5.2 \times 10^{7} \mathrm{M}^{-1} \mathrm{~s}^{-1} ; k_{7}, 6.0 \times 10^{6} \mathrm{M}^{-1} \mathrm{~s}^{-1}\right.$; $\left.k_{8}, 4.4 \times 10^{8} \mathrm{M}^{-1} \mathrm{~s}^{-1}\right)$.

Table 2.1 Absorption data.

\begin{tabular}{llll}
\hline Compound & Absorption $\boldsymbol{\lambda}_{\text {max }} / \mathbf{n m}\left(\boldsymbol{\varepsilon} / \mathbf{M}^{-1} \mathbf{c m}^{-1}\right)$ & $\boldsymbol{\varepsilon}_{\lambda=730} / \mathbf{M}^{-1} \mathbf{c m}^{-1}$ & Reference \\
\hline $\mathrm{Ru}(\text { bpy })_{3}{ }^{2+}$ & $450(12433)$ & 0 & $\mathrm{a}$ \\
$\mathrm{Ru}(\mathrm{bpy})_{3}{ }^{3+}$ & $675(409)$ & 267 & $\mathrm{~b}$ \\
$\mathrm{MV} \cdot+$ & $395(26936), 605(10620)$ & 1932 & $\mathrm{a}$ \\
$\mathrm{Co}\left(\mathrm{dpgBF}_{2}\right)_{2} \mathrm{~L}_{2}$ & $464(5600)$ & 0 & $\mathrm{a}$ \\
{$[\mathrm{Na}]\left[\mathrm{Co}\left(\mathrm{dpgBF}_{2}\right)_{2} \mathrm{~L}\right]$} & $686(10252)$ & 7506 & $\mathrm{a}$ \\
$\mathrm{MeCo}\left(\mathrm{dpgBF}_{2}\right)_{2} \mathrm{~L}$ & $406(6262)$ & 0 & $\mathrm{a}$ \\
\hline
\end{tabular}

Absorption coefficients measured in $\mathrm{CH}_{3} \mathrm{CN}$. $\mathrm{L}=\mathrm{CH}_{3} \mathrm{CN}$. a. this work. b. Reference 12 .

$$
\begin{aligned}
\frac{d R u^{2+*}}{d t} & =-k_{1}\left[R u^{2+*}\right]-k_{2}\left[R u^{2+*}\right]\left[M V^{2+}\right] \\
\frac{d M V^{2+}}{d t} & =-k_{2}\left[R u^{2+*}\right]\left[M V^{2+}\right]+k_{3}\left[R u^{3+}\right]\left[M V^{+\bullet}\right]+k_{4}\left[C o^{2+}\right]\left[M V^{+\bullet}\right]+k_{7}\left[C o^{3+}\right]\left[M V^{+\bullet}\right] \\
\frac{d R u^{3+}}{d t} & =k_{2}\left[R u^{2+*}\right]\left[M V^{2+}\right]-k_{3}\left[R u^{3+}\right]\left[M V^{+\bullet}\right]-k_{5}\left[C o^{+}\right]\left[R u^{3+}\right]-k_{6}\left[R u^{3+}\right]\left[C o^{2+}\right] \\
\frac{d M V^{+\bullet}}{d t} & =k_{2}\left[R u^{2+*}\right]\left[M V^{2+}\right]-k_{3}\left[R u^{3+}\right]\left[M V^{+\bullet}\right]-k_{4}\left[C o^{2+}\right]\left[M V^{+\bullet}\right]-k_{7}\left[C o^{3+}\right]\left[M V^{+\bullet}\right] \\
\frac{d C o^{2+}}{d t} & =-k_{4}\left[C o^{2+}\right]\left[M V^{+\bullet}\right]+k_{5}\left[C o^{+}\right]\left[R u^{3+}\right]-k_{6}\left[R u^{3+}\right]\left[\mathrm{Co}^{2+}\right]+k_{7}\left[\mathrm{Co}^{3+}\right]\left[M V^{+\bullet}\right]+2 k_{8}\left[\mathrm{Co}^{3+}\right]\left[\mathrm{Co}^{+}\right] \\
\frac{d C o^{+}}{d t} & =k_{4}\left[C o^{2+}\right]\left[M V^{+\bullet}\right]-k_{5}\left[C o^{+}\right]\left[R u^{3+}\right]-k_{8}\left[\mathrm{Co}^{3+}\right]\left[\mathrm{Co}^{+}\right] \\
\frac{d C o^{3+}}{d t} & =k_{6}\left[R u^{3+}\right]\left[\mathrm{Co}^{2+}\right]-k_{7}\left[\mathrm{Co}^{3+}\right]\left[M V^{+\bullet}\right]-k_{8}\left[\mathrm{Co}^{3+}\right]\left[\mathrm{Co}^{+}\right]
\end{aligned}
$$

Scheme 2.3 Differential equations describing the changes in concentration of ET complexes and intermediates. 


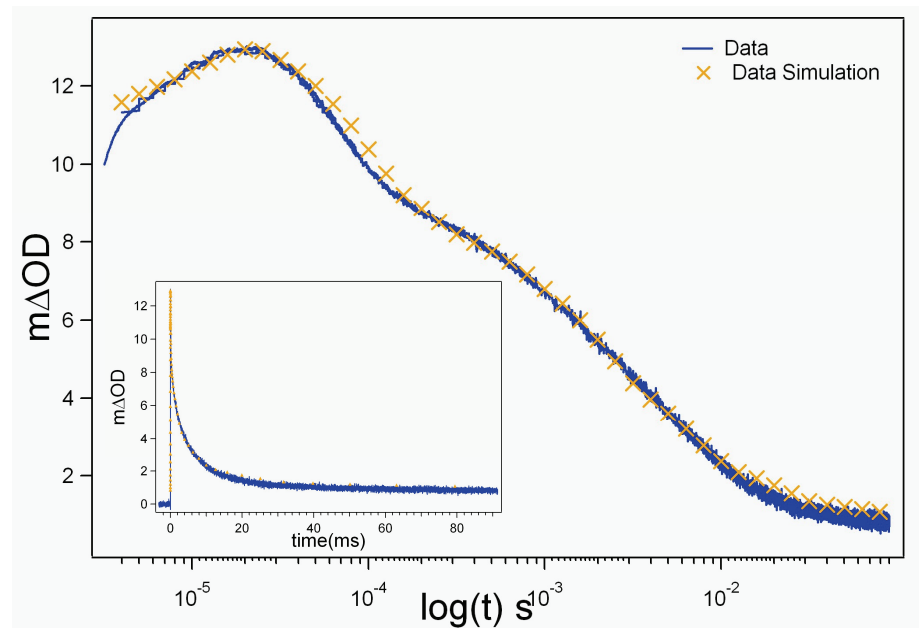

Figure 2.8 Kinetics trace of $20 \mu \mathrm{M}\left[\mathrm{Ru}(\mathrm{bpy})_{3}\right]\left[\mathrm{PF}_{6}\right]_{2}, 3.5 \mathrm{mM}$ $[\mathrm{MV}]\left[\mathrm{PF}_{6}\right]_{2}, 0.1 \mathrm{M} \mathrm{NBu}_{4} \mathrm{PF}_{6}, 125 \mu \mathrm{M} \mathrm{Co}\left(\mathrm{dmgBF}_{2}\right)_{2}\left(\mathrm{CH}_{3} \mathrm{CN}\right)_{2}$, and the simulated fit in logarithmic time. $\lambda_{\mathrm{ex}}=480 \mathrm{~nm}, \lambda_{\mathrm{obs}}=730 \mathrm{~nm}$. ET rate constants used in the simulation: $k_{1}, 1.04 \times 10^{6} \mathrm{~s}^{-1} ; k_{2}, 1.58$ × $10^{9} \mathrm{M}^{-1} \mathrm{~s}^{-1} ; k_{3}, 5.7 \times 10^{9} \mathrm{M}^{-1} \mathrm{~s}^{-1} ; k_{4}, 2.0 \times 10^{8} \mathrm{M}^{-1} \mathrm{~s}^{-1} ; k_{5}, 9.8 \times 10^{9}$ $\mathrm{M}^{-1} \mathrm{~s}^{-1} ; k_{6}, 5.2 \times 10^{7} \mathrm{M}^{-1} \mathrm{~s}^{-1} ; k_{7}, 6.0 \times 10^{6} \mathrm{M}^{-1} \mathrm{~s}^{-1} ; k_{8}, 4.4 \times 10^{8} \mathrm{M}^{-1} \mathrm{~s}^{-1}$. Inset: kinetics and fit plotted on a linear time axis.

\section{Rate Constants and Reorganization Energies}

The rate constants for electron-exchange reactions of $\left[\mathbf{C} \mathbf{o}^{\mathrm{III}}\right]$ and $\left[\mathbf{C} \mathbf{o}^{\mathrm{II}}\right]$ as well as $\left[\mathbf{C o}^{\mathrm{II}}\right]$ and [Co'] can be estimated using the Marcus cross relation, ${ }^{13} k_{12}=\left(k_{11} k_{22} K_{12} f_{12}\right)^{1 / 2}$, where $k_{12}$ describes the rate constant of an ET cross reaction, $k_{11}$ and $k_{22}$ are the self-exchange reaction rate constants, $K_{12}$ is the equilibrium constant for the cross reaction, $f_{12}$ is a known function of these parameters, $\ln f_{12}=\frac{\left(\ln K_{12}\right)^{2}}{4 \ln \left(\left(k_{11} k_{22}\right) / Z^{2}\right)}$, and $\mathrm{Z}$ is the collision frequency, estimated to be $10^{11} \mathrm{M}^{-1} \mathrm{~s}^{-1}$.

To solve for the self-exchange rate constants of the cobalt redox pairs, the specific rate for $\left[\mathrm{Ru}(\mathrm{bpy})_{3}\right]^{3+/ 2+}$ self-exchange was taken from literature data. There are two generally accepted values for this rate constant; $2.0 \times 10^{9} \mathrm{M}^{-1} \mathrm{~s}^{-1}$ at $25{ }^{\circ} \mathrm{C}$ in $1 \mathrm{M} \mathrm{HClO}_{4}(\mathrm{aq})$ reported 
by Meyer and coworkers ${ }^{14}$ and $8.3 \times 10^{6} \mathrm{M}^{-1} \mathrm{~s}^{-1}$ at $25{ }^{\circ} \mathrm{C}$ in $\mathrm{CH}_{3} \mathrm{CN}$ obtained by Chan and Wahl. ${ }^{15}$ Temperature-dependent measurements allow us to extrapolate the latter value to $7.3 \times 10^{6} \mathrm{M}^{-1} \mathrm{~s}^{-1}$ at $20^{\circ} \mathrm{C}$. Using these rate constants as limiting values, we estimated a range for the self-exchange rate constants for $\left[\mathbf{C o}^{\mathrm{III}}\right]-\left[\mathbf{C o}^{\mathrm{II}}\right]$ and $\left[\mathbf{C o}^{\mathrm{II}}\right]-\left[\mathbf{C o}^{\mathrm{I}}\right]$.

The cross reaction of $\left[\mathrm{Ru}(\mathrm{bpy})_{3}\right]^{3+}+\mathrm{MV}^{\bullet+} \rightarrow\left[\mathrm{Ru}(\mathrm{bpy})_{3}\right]^{2+}+\mathrm{MV}^{2+}\left(k_{3}\right)$ gave self-exchange rate constants for $\mathrm{MV}^{2+/{ }^{++}}\left(6.2 \times 10^{2}\right.$ and $\left.1.7 \times 10^{5} \mathrm{M}^{-1} \mathrm{~s}^{-1}\right)$ that were three to six orders of magnitude smaller than the literature value $\left(5.4 \times 10^{8} \mathrm{M}^{-1} \mathrm{~s}^{-1}\right) .{ }^{16}$ The deviation can be attributed to the fact that the Marcus cross relation does not take into account inverted driving force effects or diffusion limits. For later calculations, $k_{11}$ for $\mathrm{MV}^{2+\iota_{+}+}$was assigned the literature value. ${ }^{16}$ The self-exchange rate constants calculated for $\left[\mathbf{C o}^{\mathrm{III}}\right]-\left[\mathbf{C o}^{\mathrm{II}}\right]$ and $\left[\mathbf{C o}^{\mathrm{II}}\right]-\left[\mathbf{C o}^{\mathrm{I}}\right]$ are set out in Table 2.2. The reorganization parameter, $\lambda$, is estimated from self-exchange rate constants via $k_{11}=Z e^{\left(\frac{-\lambda_{11}}{4 R T}\right)}$, where $\mathrm{R}$ is the molar gas constant, and $\mathrm{T}$ is temperature. 
Table 2.2 Reaction parameters for $\mathrm{Co}\left(\mathrm{dpgBF}_{2}\right)_{2}\left(\mathrm{CH}_{3} \mathrm{CN}\right)_{2}$.

\begin{tabular}{|c|c|c|c|c|c|c|c|c|}
\hline $\begin{array}{l}\text { cross } \\
\text { rxn }\end{array}$ & $\begin{array}{l}k_{11} \\
\text { reaction }\end{array}$ & $\begin{array}{l}k_{22} \\
\text { reaction }\end{array}$ & $\begin{array}{l}\Delta \mathrm{E} \\
(\mathrm{V})^{\mathrm{a}}\end{array}$ & $\mathrm{K}_{12}$ & $\begin{array}{l}k_{12} \\
\left(\mathrm{M}^{-1} \mathrm{~s}^{-1}\right)\end{array}$ & $\left(\mathrm{M}^{-1} \mathrm{~s}^{-1}\right)$ & $\begin{array}{l}k_{22} \\
(\text { calc'd) } \\
\left(\mathrm{M}^{-1} \mathrm{~s}^{-1}\right)\end{array}$ & $\lambda(\mathrm{eV})$ \\
\hline \multirow[t]{2}{*}{6} & $\mathrm{Ru}(\mathrm{bpy})_{3}{ }^{3+/ 2+}$ & $\mathrm{Co}^{\mathrm{III}} / \mathrm{Co}^{\mathrm{II}}$ & 0.97 & $4.74 \times 10^{16}$ & $5.2 \times 10^{7}$ & $2.0 \times 10^{9 b}$ & $9.5 \times 10^{-8}$ & 4.19 \\
\hline & & & & & & $7.3 \times 10^{6 c}$ & $2.6 \times 10^{-5}$ & 3.60 \\
\hline \multirow[t]{2}{*}{7} & $\mathrm{MV}^{2+/+}$ & $\mathrm{Co}^{\mathrm{III}} / \mathrm{Co}^{\mathrm{II}}$ & 0.76 & $1.16 \times 10^{13}$ & $6.0 \times 10^{6}$ & $5.4 \times 10^{8 \mathrm{~d}}$ & $9.3 \times 10^{-7}$ & 3.97 \\
\hline & & & & & & & Avg. & $3.9 \pm 0.3$ \\
\hline \multirow[t]{2}{*}{3} & $\mathrm{Ru}(\mathrm{bpy})_{3}{ }^{3+/ 2+}$ & $\mathrm{MV}^{2+/+}$ & 1.73 & $5.52 \times 10^{29}$ & $5.7 \times 10^{9}$ & $2.0 \times 10^{9 b}$ & $6.2 \times 10^{2}$ & 1.91 \\
\hline & & & & & & $7.3 \times 10^{6 c}$ & $1.7 \times 10^{5}$ & 1.34 \\
\hline 4 & $\mathrm{MV}^{2+/+}$ & $\mathrm{Co}^{\mathrm{II}} / \mathrm{Co}^{\mathrm{I}}$ & 0.18 & $1.24 \times 10^{3}$ & $2 \times 10^{8}$ & $5.4 \times 10^{8 \mathrm{~d}}$ & $1.2 \times 10^{5 \mathrm{e}}$ & $1.38^{\mathrm{f}}$ \\
\hline
\end{tabular}

a. See Table 2.3 b. Reference 14. c. Reference 15. d. Reference 16. e. Estimated uncertainty \pm 0.5 $\mathrm{M}^{-1} \mathrm{~s}^{-1} \mathrm{f}$. Estimated uncertainty $\pm 0.05 \mathrm{eV}$.

Table 2.3 Reduction potentials (V. vs. SCE) in acetonitrile.

\begin{tabular}{ll}
\hline Compound & $E^{\circ /} / \mathrm{V}$ \\
\hline $\mathrm{Ru}(\mathrm{bpy})_{3}{ }^{3+/ 2+}$ & $1.27^{\mathrm{a}}$ \\
$\mathrm{Co}(\mathrm{dpgBF})_{2}\left(\mathrm{CH}_{3} \mathrm{CN}\right)_{2}{ }^{+/ 0}$ & $\sim 0.3^{\mathrm{b}, \mathrm{c}}$ \\
$\mathrm{Co}\left(\mathrm{dpgBF}_{2}\right)_{2}\left(\mathrm{CH}_{3} \mathrm{CN}\right)_{2}{ }^{0 /-}$ & $-0.28^{\mathrm{c}}$ \\
$\mathrm{MV}^{2+/+}$ & $-0.46^{\mathrm{d}}$ \\
\hline
\end{tabular}

a. Reference 17 b. Irreversible couple c. Reference 5. d. Reference 18. 
Thermodynamic Analysis

The driving forces $\left(-\Delta G^{\circ}\right)$ for the elementary steps associated with $\mathrm{H}_{2}$ evolution were calculated as described in Figure 2.9 and Table 2.4. The driving force for proton reduction $\left(-\Delta G_{R}^{\circ}\right)$ is the difference between the standard potential for hydrogen evolution by proton donor $\mathrm{HA}^{19}\left(E^{\circ}\left(\mathrm{HA} / \mathrm{H}_{2}\right)\right)$ and the reduction potential of the reducing equivalent, $\mathrm{D}$. The driving force for reduction of $\left[\mathrm{Co}^{\mathrm{II}}\right]$ by reductant $\mathrm{D}$ is described by $-\Delta G_{1}^{\circ}$ and $-\left(\Delta G_{2}^{\circ}-\right.$ $\left.\Delta G_{1}^{\circ}\right)$. The driving force for protonation of $\left[\mathbf{C o}^{\mathrm{I}}\right]\left(-\left(\Delta G_{2 a}^{\circ}-\Delta G_{1}^{\circ}\right),-\left(\Delta G_{3}^{\circ}-\Delta G_{2}^{\circ}\right)\right.$, and $\left.-\left(\Delta G_{4}^{\circ}-\Delta G_{3}^{\circ}\right)\right)$ is proportional to the $\mathrm{p} K_{a}$ difference between $\mathrm{HA}$ and $\mathrm{Co}^{\mathrm{III}} \mathrm{H}$. With sufficiently powerful reductants and proton sources, these steps are exergonic.

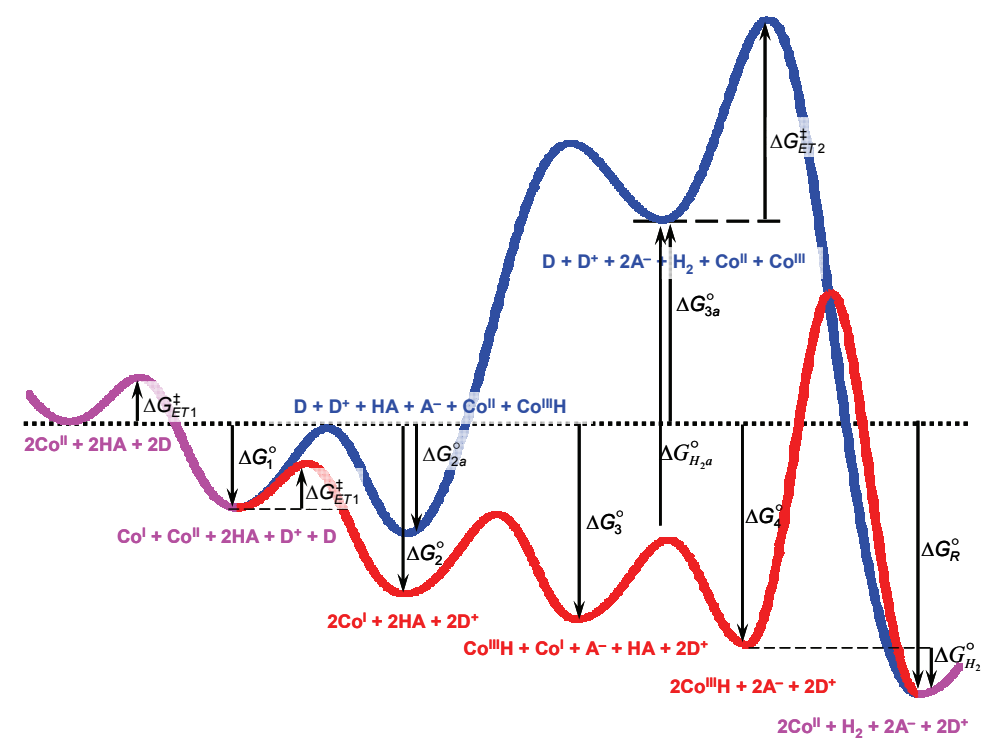

Figure 2.9 Thermodynamic analysis of the homolytic and heterolytic pathways for hydrogen evolution. D is a reductant. Values for barriers and driving forces are given in Table 2.4. 
Table 2.4 Barriers and driving forces associated with $\mathrm{H}_{2}$ evolution.

\begin{tabular}{|c|c|}
\hline$\Delta G_{1}^{\circ}$ & $\mathfrak{J}\left[E_{\mathrm{D}^{+} / \mathrm{D}}^{\circ}-E_{\mathrm{Co}^{\prime \prime} / \mathrm{Co}^{\prime}}^{\circ}\right]$ \\
\hline$\Delta G_{2}^{\circ}$ & $\Delta G_{1}^{\circ}+\mathfrak{J}\left[E_{\mathrm{D}^{+} / \mathrm{D}}^{\circ}-E_{\mathrm{Co}^{\prime \prime} / \mathrm{Co}^{\prime}}^{\circ}\right]$ \\
\hline$\Delta G_{3}^{\circ}$ & $\Delta G_{2}^{\circ}+2.303 R T\left[p K_{a}(\mathrm{HA})-p K_{a}\left(\mathrm{Co}^{\prime \prime \prime} \mathrm{H}\right)\right]$ \\
\hline$\Delta G_{4}^{\circ}$ & $\Delta G_{3}^{\circ}+2.303 R T\left[p K_{a}(\mathrm{HA})-p K_{a}\left(\mathrm{Co}^{\prime \prime \prime} \mathrm{H}\right)\right]$ \\
\hline$\Delta G_{R}^{\circ}$ & $2 \mathfrak{I}\left[E_{\mathrm{D}^{+} / \mathrm{D}}^{\circ}-E_{\mathrm{HA} / \mathrm{H}_{2}}^{\circ}\right]$ \\
\hline$\Delta G_{\mathrm{H}_{2}}^{\circ}$ & $\begin{array}{l}2 \mathfrak{I}\left[E_{\mathrm{Co} / \mathrm{Co}}^{\circ}-E_{\mathrm{HA} / \mathrm{H}_{2}}^{\circ}\right]-2(2.303 R T)\left[p K_{a}(\mathrm{HA})-p K_{a}\left(\mathrm{Co}^{\prime \prime \prime} \mathrm{H}\right)\right] \\
\Delta G_{R}^{\circ}-\Delta G_{4}^{\circ}\end{array}$ \\
\hline$\Delta G_{2 a}^{\circ}$ & $\Delta G_{1}^{\circ}+2.303 R T\left[p K_{a}(\mathrm{HA})-p K_{a}\left(\mathrm{Co}^{\prime \prime \prime} \mathrm{H}\right)\right]$ \\
\hline$\Delta G_{3 a}^{\circ}$ & 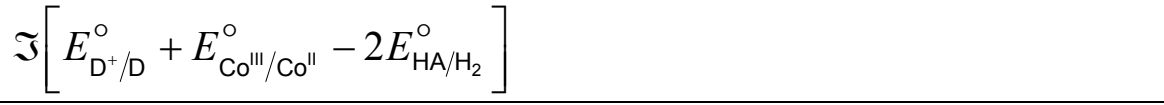 \\
\hline$\Delta G_{\mathrm{H}_{2} a}^{\circ}$ & $\begin{array}{l}\mathfrak{J}\left[E_{\mathrm{Co}^{\prime \prime} / \mathrm{Co}^{\prime}}^{\circ}+E_{\mathrm{Co}^{\prime \prime \prime} / \mathrm{Co}^{\prime \prime}}^{\circ}-2 E_{\mathrm{HA} / \mathrm{H}_{2}}^{\circ}\right]-2.303 R T\left[p K_{a}(\mathrm{HA})-p K_{a}\left(\mathrm{Co}^{\prime \prime \prime} \mathrm{H}\right)\right] \\
\Delta G_{3 a}^{\circ}-\Delta G_{2 a}^{\circ}\end{array}$ \\
\hline$\Delta G_{E T 1}^{\ddagger}$ & $\frac{\left(\mathfrak{I}\left[E_{\mathrm{D}^{+} / \mathrm{D}}^{\circ}-E_{\mathrm{Co}^{\prime \prime} / \mathrm{Co}^{\prime}}^{\circ}\right]+\frac{1}{2}\left(\lambda_{\mathrm{Co}^{\prime \prime} / \mathrm{Co}^{\prime}}+\lambda_{\mathrm{D}^{+} / \mathrm{D}}\right)\right)^{2}}{2\left(\lambda_{\mathrm{Co}^{\prime \prime} / \mathrm{Co}^{\prime}}+\lambda_{\mathrm{D}^{+} / \mathrm{D}}\right)}$ \\
\hline$\Delta G_{E T 2}^{\ddagger}$ & $\frac{\left(\mathfrak{I}\left[E_{\mathrm{D}^{+} / \mathrm{D}}^{\circ}-E_{\mathrm{Co}^{\prime \prime \prime} / \mathrm{Co}^{\prime \prime}}^{\circ}\right]+\frac{1}{2}\left(\lambda_{\mathrm{Co}^{\prime \prime \prime} / \mathrm{Co}^{\prime \prime \prime}}+\lambda_{\mathrm{D}^{+} / \mathrm{D}}\right)\right)^{2}}{2\left(\lambda_{\mathrm{Co}^{\prime \prime \prime} / \mathrm{Co}^{\prime \prime}}+\lambda_{\mathrm{D}^{+} / \mathrm{D}}\right)}$ \\
\hline
\end{tabular}

$F=$ Faraday's constant, $96485.31 \mathrm{C} \mathrm{mol}^{-1}, \mathrm{R}=$ ideal gas constant, $8.31441 \mathrm{~J} \mathrm{~K}^{-1} \mathrm{~mol}^{-1}, \mathrm{~T}=$ temperature, $293.15 \mathrm{~K}$.

In the homolytic reaction pathway, the driving force for the elementary step for $\mathrm{H}_{2}$ evolution is $-\Delta G_{\mathrm{H}_{2}}^{\circ}$, the difference between $\Delta G_{R}^{\circ}$ and $\Delta G_{4}^{\circ}$. The driving force for the elementary $\mathrm{H}_{2}$ formation step in the heterolytic proton reduction pathway $\left(\Delta G_{\mathrm{H}_{2} a}^{\circ}\right)$ depends on the average of $E^{\circ}\left(\mathbf{C o}^{\mathrm{III} / \mathrm{II}}\right)$ and $E^{\circ}\left(\mathbf{C o}^{\mathrm{II} / \mathrm{I}}\right)$, relative to the proton reduction 
potential, and the $\mathrm{p} K_{a}$ difference of $\mathrm{HA}$ and $\mathrm{Co}^{\mathrm{III}} \mathrm{H}$. To estimate a lower limit for $\Delta G_{\mathrm{H}_{2} a}^{\circ}$,

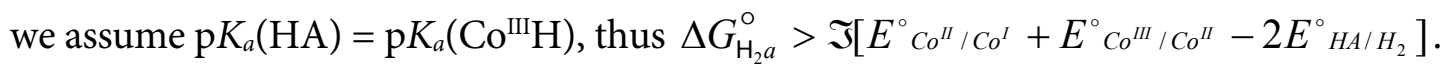

The barriers for electron transfer from D to $\left[\mathbf{C o}^{\mathrm{II}}\right]\left(\Delta G_{E T 1}^{\ddagger}\right)$ and D to $\left[\mathbf{C o}^{\mathrm{III}}\right]\left(\Delta G_{E T 2}^{\ddagger}\right)$ depend both on the driving forces and reorganization parameters. We assume $E^{\circ}\left(\mathrm{D}^{+/ 0}\right)=$ $E^{\circ}\left(\mathbf{C} \mathbf{o}^{\mathrm{II} / \mathrm{I}}\right)$ and a range of self-exchange reorganization energies for the $\mathrm{D}^{+/ 0}$ couple $\left(\lambda_{11}=0-\right.$ $1.5 \mathrm{eV})$. We take $\lambda_{22}\left(\mathbf{C} \mathbf{o}^{\mathrm{II} / \mathrm{I}}\right)=1.4 \mathrm{eV}$ and $\lambda_{22}\left(\mathbf{C} \mathbf{o}^{\mathrm{III} / \mathrm{II}}\right)=3.9 \mathrm{eV}$, as calculated above, and the reorganization energy for the cross reactions as $\lambda_{12}=1 / 2\left(\lambda_{11}+\lambda_{22}\right)$. The activation freeenergy change for reduction of $\left[\mathbf{C o}^{\mathrm{II}}\right]$ is $\Delta G^{\ddagger}\left(\mathbf{C o}^{\mathrm{II} / \mathrm{I}}\right)=\lambda_{12}\left(\mathbf{C o}^{\mathrm{II} / \mathrm{I}}\right) / 4\left(4-8 \mathrm{kcal} \mathrm{mol}^{-1}\right)$ and that for reduction of $\left[\mathbf{C o}^{\mathrm{III}}\right]$ is $\Delta G^{\ddagger}\left(\mathbf{C o}^{\mathrm{III} / \mathrm{II}}\right)=\left[-0.5+\lambda_{12}\left(\mathbf{C o}^{\mathrm{III} / \mathrm{II}}\right)\right]^{2} /\left(4 \lambda_{12}\left(\mathbf{C o}^{\mathrm{III} / \mathrm{II}}\right)\right)\left(6-10 \mathrm{kcal} \mathrm{mol}^{-1}\right.$, $-\Delta G^{\circ}$ for reduction of $\left[\mathbf{C o}^{\mathrm{III}}\right]$ is $\left.-0.5 \mathrm{eV}\right)$. The relative reaction rates for these two ET processes are given by $k\left(\mathbf{C} \mathbf{o}^{\mathrm{II} / \mathrm{I}}\right) / k\left(\mathbf{C} \mathbf{o}^{\mathrm{III} / \mathrm{II}}\right)=\exp \left(\left[\Delta G^{\ddagger}\left(\mathbf{C} \mathbf{o}^{\mathrm{III} / \mathrm{II}}\right)-\Delta G^{\ddagger}\left(\mathbf{C} \mathbf{o}^{\mathrm{II} / \mathrm{I}}\right)\right] / \mathrm{RT}\right)=35$ at 295 K.

\section{Discussion}

TA spectroscopy demonstrates that $\mathrm{MV}^{2+}$ oxidatively quenches pulsed-laser excited $\left[\mathrm{Ru}(\mathrm{bpy})_{3}\right]^{2+}$ to form $\mathrm{MV}^{\bullet+}$, which delivers an electron to $\left[\mathrm{Co}^{\mathrm{II}}\right]$ producing anionic $\left[\mathrm{Co}^{\mathrm{I}}\right]$ and $\left[\mathrm{Ru}(\text { bpy })_{3}\right]^{3+}$. $\left[\mathrm{Ru}(\text { bpy })_{3}\right]^{3+}$ oxidizes both $\left[\mathbf{C o}^{\mathrm{I}}\right]$ and $\left[\mathbf{C o}^{\mathrm{II}}\right]$, and the $\left[\mathbf{C o}^{\mathrm{III}}\right]$, although formed irreversibly on the electrochemical timescale (possibly owing to changes in ligation $),{ }^{5}$ is reduced back to $\left[\mathbf{C o}^{\mathrm{II}}\right]$ by the remaining reductants in solution, $\left[\mathrm{Co}^{\mathrm{I}}\right]$ and $\mathrm{MV}^{\bullet+}$. Global analysis supports these assignments, as the TA spectra can be modeled as linear combinations of steady-state absorption spectra of the starting compounds and the transiently formed ET species. Kinetics traces (Figure 2.7, Figure 2.8) measured at $730 \mathrm{~nm}$ provide information about ET in the system. The rate law for the coupled series of ET 
reactions (Scheme 2.2, Scheme 2.3) was solved numerically to produce time-dependent concentration profiles for all species which, with molar extinction coefficients and selected independently determined rate constants, allowed us to simulate the observed kinetics and extract elementary rate constants. The specific rate for $\left[\mathbf{C o}^{\mathrm{II}}\right]$ reduction by $\mathrm{MV}^{\bullet+}$ is $2.0 \times 10^{8}$ $\mathrm{M}^{-1} \mathrm{~s}^{-1}$ and those for $\left[\mathrm{Co}^{\mathrm{I}}\right]$ oxidations by $\left[\mathrm{Ru}(\text { bpy })_{3}\right]^{3+}$ and $\left[\mathrm{Co}^{\mathrm{III}}\right]$ are $9.8 \times 10^{9} \mathrm{M}^{-1} \mathrm{~s}^{-1}$ and 4.4 $\times 10^{8} \mathrm{M}^{-1} \mathrm{~s}^{-1}$, respectively. Rate constants for two other ET reactions were extracted from the simulations: $\left[\mathrm{Co}^{\mathrm{II}}\right]$ oxidation by $\left[\mathrm{Ru}(\mathrm{bpy})_{3}\right]^{3+}\left(5.2 \times 10^{7} \mathrm{M}^{-1} \mathrm{~s}^{-1}\right)$; and $\left[\mathrm{Co}^{\mathrm{III}}\right]$ reduction by $\mathrm{MV}^{\bullet+}\left(6.0 \times 10^{6} \mathrm{M}^{-1} \mathrm{~s}^{-1}\right)$.

The specific rates of $\left[\mathbf{C o}^{\mathrm{III}}\right]-\left[\mathbf{C o}^{\mathrm{II}}\right]$ and $\left[\mathrm{Co}^{\mathrm{II}}\right]-\left[\mathbf{C o}{ }^{\mathrm{I}}\right]$ electron self-exchange reactions can be estimated using the Marcus cross relation. ${ }^{13}$ Taking the rate constants for reductions of $\left[\mathrm{Ru}(\text { bpy })_{3}\right]^{3+}$ by $\left[\mathbf{C o}^{\mathrm{II}}\right]$ and $\left[\mathrm{Co}^{\mathrm{III}}\right]$ by $\mathrm{MV}^{\bullet+}$, we estimate that the $\left[\mathbf{C o}{ }^{\mathrm{III}}\right]-\left[\mathbf{C o} \mathbf{o}^{\mathrm{II}}\right]$ rate constant is between $9.5 \times 10^{-8}$ and $2.6 \times 10^{-5} \mathrm{M}^{-1} \mathrm{~s}^{-1}$. This value is lower than that estimated for $\left[\mathrm{Co}^{\text {III/III }}\left(\mathrm{dmgBF}_{2}\right)_{2}\left(\mathrm{H}_{2} \mathrm{O}\right)_{2}\right]^{+/ 0}$ in aqueous solution $\left(1.7 \times 10^{-4} \mathrm{M}^{-1} \mathrm{~s}^{-1}\right.$ to $\left.8.7 \times 10^{-3} \mathrm{M}^{-1} \mathrm{~s}^{-1}\right){ }^{20}$ Corresponding self-exchange rate constants for $\left[\mathrm{Co}^{\mathrm{III} / \mathrm{II}}\left(\mathrm{N}_{4}\right)\left(\mathrm{H}_{2} \mathrm{O}\right)_{2}\right]^{3+/ 2+}$ complexes with equatorial tetraaza macrocyclic ligands $\left(4.5 \times 10^{-5} \mathrm{M}^{-1} \mathrm{~s}^{-1}\right.$ to $\left.5.0 \times 10^{-2} \mathrm{M}^{-1} \mathrm{~s}^{-1}\right)$ correlate with structural changes, particularly variations in axial $\mathrm{Co}-\mathrm{OH}_{2}$ bond lengths. ${ }^{21}$ The rate constant estimated for $\left[\mathbf{C o}^{\mathrm{III}}\right]-\left[\mathbf{C o}^{\mathrm{II}}\right]$ is near the low end of the range for low-spin $\mathrm{Co}^{\mathrm{III} / \mathrm{II}}$ complexes in aqueous solution.

Using the rate constants for $\left[\mathbf{C o}^{\mathrm{II}}\right]$ reduction by $\mathrm{MV}^{\bullet+}$, we can estimate the $\left[\mathrm{Co}^{\mathrm{II}}\right]-\left[\mathbf{C o}^{\mathrm{I}}\right]$ selfexchange rate constant to be approximately $1.2( \pm 0.5) \times 10^{5} \mathrm{M}^{-1} \mathrm{~s}^{-1}$. While we determined the rate constant for $\left[\mathrm{Co}^{\mathrm{I}}\right]$ oxidation by $\left[\mathrm{Ru}(\mathrm{bpy})_{3}\right]^{3+}$, the large driving force for this reaction $(1.55 \mathrm{eV})$ places it in the Marcus inverted region where calculation of self-exchange rate constants is unreliable. The stark difference between the $\left[\mathbf{C o}^{\mathrm{III}}\right]-\left[\mathbf{C o}^{\mathrm{II}}\right]$ and $\left[\mathrm{Co}^{\mathrm{II}}\right]-\left[\mathbf{C o} \mathbf{o}^{\mathrm{I}}\right]$ rates is similar to that found for $\left[\mathrm{Co}^{\mathrm{II}}(\mathrm{bpy})_{3}\right]^{2+}\left(\left[\mathrm{Co}^{\mathrm{III} / \mathrm{II}}(\mathrm{bpy})_{3}\right]^{3+/ 2+}, 18 \mathrm{M}^{-1} \mathrm{~s}^{-1} ;\left[\mathrm{Co}^{\mathrm{II} / \mathrm{I}}(\mathrm{bpy})_{3}\right]^{2+/+}\right.$, 
$\left.\sim 10^{9} \mathrm{M}^{-1} \mathrm{~s}^{-1}\right) .{ }^{22}$ The reactivity differences are attributable to the substantial nuclear configuration changes that accompany $\left[\mathrm{Co}^{\mathrm{III} / \mathrm{II}}(\mathrm{bpy})_{3}\right]^{3+/ 2+}$ exchange $\left(\Delta \mathrm{d}_{0}\right.$ for Co- $\mathrm{N}$ bonds $=$ $0.19 \AA)$, and the modest rearrangements upon $\left[\mathrm{Co}^{\mathrm{II}}(\mathrm{bpy})_{3}\right]^{2+}$ reduction to $\left[\mathrm{Co}^{\mathrm{I}}(\mathrm{bpy})_{3}\right]^{+}\left(\Delta \mathrm{d}_{0}\right.$ $=-0.02 \AA)$. It is interesting to note that a pronounced change is found in the solid-state structures of $\left[\mathbf{C o}^{\mathrm{II}}\right]$ and $\left[\mathrm{Co}^{\mathrm{I}}\right]$ : an axial $\mathrm{CH}_{3} \mathrm{CN}$ ligand is lost upon reduction, accompanied by a 0.268 - $\AA$ shortening of the remaining nitrile bond, $0.27-\AA$ displacement of the $\mathrm{Co}^{\mathrm{I}}$ ion, and a $0.035-\AA$ reduction of the $\mathrm{Co}-\mathrm{N}_{\text {imine }}$ distance. ${ }^{5}$ These considerable structural changes are at variance with the large $\left[\mathbf{C o}^{\mathrm{II}}\right]-\left[\mathbf{C o}^{\mathrm{I}}\right]$ electron self-exchange rate constants extracted using the cross relation. The discrepancy may be an indication that electron transfer and the large structural rearrangements occur in separate elementary reaction steps. The large nuclear rearrangements expected for oxidation of $\left[\mathbf{C o}^{\mathrm{II}}\right]$ to $\left[\mathbf{C} \mathbf{o}^{\mathrm{II}}\right]$, however, are reflected in the $\left[\mathbf{C o}{ }^{\mathrm{III}}\right]-\left[\mathbf{C o}{ }^{\mathrm{II}}\right]$ self-exchange rate constant. ${ }^{20}$

The reorganization parameter, $\lambda$, which accounts for both inner- and outer-sphere rearrangements accompanying an ET process, can be estimated from self-exchange rates. The reorganization energy for $\left[\mathbf{C o}^{\mathrm{III}}\right]-\left[\mathbf{C o}^{\mathrm{II}}\right]$ self-exchange is approximately $3.9( \pm 0.3) \mathrm{eV}$ (89.9 $\left.( \pm 6.9) \mathrm{kcal} \mathrm{mol}^{-1}\right)$, while that for the $\left[\mathbf{C o}^{\mathrm{II}}\right]-\left[\mathbf{C o}^{\mathrm{I}}\right]$ reaction is substantially smaller, about $1.4( \pm 0.05) \mathrm{eV}\left(34.6( \pm 1.2) \mathrm{kcal} \mathrm{mol}^{-1}\right)$. These calculations emphasize the formidable energy barriers associated with a hydrogen-evolving mechanism that requires a [Co $\left.{ }^{\text {III }}\right]$ intermediate.

Protonation of the reduced catalyst is described by a driving force $\left(-\left(\Delta G_{3}^{\circ}-\Delta G_{2}^{\circ}\right)\right)$ proportional to the $\mathrm{p} K_{\mathrm{a}}$ difference of the proton donor $(\mathrm{HA})$ and $\mathrm{Co}^{\mathrm{III}} \mathrm{H}$. It is worth noting that across the series of cobaloximes, the $\mathrm{Co}^{\mathrm{III}} \mathrm{H}$ pK $K_{\mathrm{a}}$ increases as $\mathrm{E}^{\mathrm{O}}\left[\mathrm{Co}^{\mathrm{II} / \mathrm{I}}\right]$ decreases, and substantially stronger acids are required to protonate the more easily reduced complexes. ${ }^{23}$ More strongly reducing $\mathrm{Co}^{\mathrm{I}}$ intermediates produce substantially stabilized $\mathrm{Co}^{\mathrm{III}} \mathrm{H}$ species 
upon protonation by strong acids. The extent of stabilization of the hydride will directly affect its tendency to produce $\mathrm{H}_{2}$. Weaker $\mathrm{Co}-\mathrm{H}$ bonds are correlated with low-barrier homolytic cleavage to eliminate $\mathrm{H}_{2}{ }^{24}$ The hydridicity of the hydride, however, will facilitate the reaction with a proton source in heterolytic $\mathrm{H}_{2}$ production. The free energy of hydride transfer depends on the hydride $\mathrm{p} K_{\mathrm{a}}$ and the $\mathrm{Co}^{\mathrm{III} / \mathrm{II}}$ and $\mathrm{Co}^{\mathrm{II/I}}$ reduction potentials: a lower $\mathrm{p} K_{\mathrm{a}}$ corresponds to a better hydride donor. ${ }^{25}$

The large reorganization energy associated with $\left[\mathbf{C o}^{\mathrm{III}}\right]-\left[\mathbf{C o}{ }^{\mathrm{II}}\right]$ electron transfer implies that the barrier to reduction of $\left[\mathbf{C o}^{\mathrm{III}}\right]$ by $\mathrm{D}\left(\Delta G_{E T 2}^{\ddagger}\right)$ is about $2.1 \mathrm{kcal} \mathrm{mol}^{-1}$ higher than that of [Co $\left.{ }^{\mathrm{II}}\right]\left(\Delta G_{E T 1}^{\ddagger}\right)$, corresponding to a $\sim 35$-fold lower specific rate. The largest barriers to catalysis, then, likely will be associated with the elementary steps that form $\mathrm{H}_{2}{ }^{26}$ Protonation of the $\mathrm{Co}^{\mathrm{III}} \mathrm{H}$ leads to release of $\mathrm{H}_{2}$ and generation of [ $\left.\mathrm{Co}^{\mathrm{III}}\right]$ in the heterolytic pathway, and the driving force for this reaction $\left(-\Delta G_{H_{2} a}^{0}\right)$, which depends on $E^{\circ}\left(\mathbf{C o}^{\mathrm{III} / \mathrm{II}}\right)$ relative to $E^{\circ}\left(\mathrm{HA} / \mathrm{H}_{2}\right)$, is in most cases thermodynamically unfavorable. Thus the barrier for this elementary step ( $\Delta G_{\mathrm{H}_{2}^{\mathrm{a}}}^{\ddagger}$ ) is estimated to be greater than $11 \mathrm{kcal} \mathrm{mol}^{-1}$, the lower limit for $\Delta G_{\mathrm{H}_{2} \mathrm{a}}^{0}$. Little is known about the rate of bimolecular reductive elimination of $\mathrm{H}_{2}$ from metal hydrides (homolytic path). Chao and Espenson obtained a second-order rate constant of $1.7 \times 10^{4} \mathrm{M}^{-1} \mathrm{~s}^{-1}$ for the reactivity of an isolable phosphine stabilized hydride species, $\mathrm{HCo}(\mathrm{dmgH})_{2} \mathrm{P}\left(n-\mathrm{C}_{4} \mathrm{H}_{9}\right)_{3 .}{ }^{27}$ This corresponds to a barrier of approximately $8-10$ $\mathrm{kcal} \mathrm{mol}^{-1}$ for the bimolecular reductive elimination of $\mathrm{H}_{2}$. As the hydride of the cobaloxime explored here is significantly more reactive, a reasonable estimate for the barrier is $5-8 \mathrm{kcal}$ $\mathrm{mol}^{-1}$.

Under the assumption of rapid electron and proton transfers such that $\left[\mathbf{C o}^{\mathrm{II}}\right],\left[\mathbf{C o}^{\mathrm{I}}\right]$, and $\mathrm{Co}^{\mathrm{III}} \mathrm{H}$ will be present at their equilibrium concentrations, we can predict that the rate of $\mathrm{H}_{2}$ 
formation $\left(d\left[\mathrm{H}_{2}\right] / d t\right)$ will be proportional to $k_{\mathrm{H}_{2}}\left[\mathrm{Co}^{\mathrm{III}} \mathrm{H}\right]^{2}$ for the homolytic pathway and to $k_{\mathrm{H}_{2} \mathrm{a}}[H A]\left[C o^{I I I} H\right]$ for the heterolytic route. Ultimately, the dominance of one path over the other during catalysis depends not just on the relative barrier heights for $\mathrm{H}_{2}$ elimination but also on the relative concentrations of $\mathrm{HA}$ and $\mathrm{Co}^{\mathrm{III}} \mathrm{H} \cdot{ }^{27}$

It was initially thought that this flash-quench system was well-poised to study the subsequent reactivity of $\left[\mathbf{C o}^{\mathrm{I}}\right]$ with a proton donor. TA and kinetics traces were measured for samples of $\left[\mathrm{Ru}(\mathrm{bpy})_{3}\right]^{2+}, \mathrm{MV}^{2+}$, and $\mathrm{Co}^{\mathrm{II}}$ in the presence of varying concentrations of $p$ toluenesulfonic acid monohydrate $\left(\mathrm{TsOH} \bullet \mathrm{H}_{2} \mathrm{O}\right)$. The $\mathrm{Co}^{\mathrm{III}} \mathrm{H}$ species postulated to form upon protonation has not been isolated, but it is believed to be a weakly absorbing species with a spectrum similar to the corresponding $\mathrm{Co}^{\mathrm{III}} \mathrm{Me}$ (Figure 2.4). As the $\mathrm{Co}^{\mathrm{III}} \mathrm{H}$ has a weak spectroscopic handle, kinetics traces monitoring the bleach of $\left[\mathbf{C o}^{\mathrm{I}}\right]$ at $730 \mathrm{~nm}$ were collected, as $[\mathbf{C o}]$ consumption corresponds to the formation of the $\left[\mathbf{C o}{ }^{I I I} \mathbf{H}\right]$. Unfortunately, measurements of $\left[\mathrm{Co}^{\mathrm{I}}\right]$ reactivity in the presence of $\mathrm{TsOH} \bullet \mathrm{H}_{2} \mathrm{O}$ were extremely irreproducible across a substantial number of experiments. Ultimately, it was determined that minor but unavoidable acid-catalyzed degradation of $\left[\mathbf{C o}^{\mathrm{II}}\right](<1 \%)$ produced enough impurities to significantly influence the reaction kinetics of the transiently generated $\left[\mathbf{C o}^{\mathrm{I}}\right]$ species. Transient measurements of the protonation process are a significant challenge-the experiment must rely on a system that avoids harsh conditions, yet substantially strong acids are necessary to protonate $\left[\mathbf{C o}^{\mathrm{I}}\right] .{ }^{5}$

\section{Conclusions}

Thermodynamic analysis of two proton reduction pathways reveals that the driving force for $\mathrm{H}_{2}$ production in the homolytic pathway depends on $E^{\circ}\left(\mathbf{C o}^{\mathrm{II} / \mathrm{I}}\right)$ (relative to $E^{\circ}\left(\mathrm{HA} / \mathrm{H}_{2}\right)$ ) and the difference in $p K_{a}$ between $\mathrm{Co}^{\mathrm{III}} \mathrm{H}$ and $\mathrm{HA}$. In the heterolytic pathway, $E^{\circ}\left(\mathbf{C o} \mathbf{o}^{\mathrm{III} / \mathrm{II}}\right)$ is 
an additional determinant that, in most cases, renders heterolytic $\mathrm{H}_{2}$ evolution extremely unfavorable. ${ }^{28}$ Furthermore, the barrier associated with reduction of transiently generated $\left[\mathbf{C o}{ }^{\mathrm{II}}\right]$ is significantly higher than that for $\left[\mathbf{C o}^{\mathrm{II}}\right]$. Though the heterolytic route can dominate at very high acid concentrations, the relatively high energy barriers and unfavorable driving forces are significant. While little is known about the barrier to the $\mathrm{Co}^{\mathrm{III}} \mathrm{H}$ bimolecular reaction, covalently linking two $\mathrm{Co}^{\mathrm{III}} \mathrm{H}$ complexes could substantially increase the rate of $\mathrm{H}_{2}$ production by decreasing the volume required for diffusional collisions.

\section{Acknowledgement}

Bruce Brunschwig, Xile Hu, Jay Labinger, and Jonas Peters are gratefully acknowledged for insightful discussions. Etsuko Fujita assisted in obtaining in situ absorption spectra. This work was supported by the NSF Center for Chemical Innovation (Powering the Planet, CHE-0802907, CHE-0947829), the Arnold and Mabel Beckman Foundation, CCSER (Gordon and Betty Moore Foundation), and the $\mathrm{BP} \mathrm{MC}^{2}$ program.

\section{Experimental Details}

\section{Reagents}

Syntheses of air and moisture sensitive compounds were carried out using Schlenk techniques or in a nitrogen atmosphere glovebox. Solvents for these syntheses were dried by a standard method ${ }^{29}$ or over activated sieves followed by passage over activated alumina. $\mathrm{CD}_{3} \mathrm{CN}$ was obtained from Cambridge Isotope Laboratories, Inc. All materials, unless noted, were used as received. Elemental analysis was performed by Columbia Analytical Services, Tucson, AZ. 
All samples were prepared in high purity (>99.99\%) $\mathrm{CH}_{3} \mathrm{CN}$ (EMD) in a high-vacuum 1 $\mathrm{cm}$ pathlength fused quartz cell (Starna Cells) connected to a $10 \mathrm{~mL}$ bulb. A typical sample was prepared as follows: in an inert atmosphere glovebox, a $2 \mathrm{~mL}$ solution of $\sim 126-314 \mu \mathrm{M}$ $\mathrm{Co}\left(\mathrm{dpgBF}_{2}\right)_{2}\left(\mathrm{CH}_{3} \mathrm{CN}\right)_{2}$ and a $2 \mathrm{~mL}$ solution of $\sim 40 \mu \mathrm{M}\left[\mathrm{Ru}(\mathrm{bpy})_{3}\right]\left[\mathrm{PF}_{6}\right]_{2}, 7 \mathrm{mM}[\mathrm{MV}]\left[\mathrm{PF}_{6}\right]_{2}$ and $0.2 \mathrm{M} \mathrm{NBu}_{4} \mathrm{PF}_{6}$ in dry, degassed $\mathrm{CH}_{3} \mathrm{CN}$ were placed into the cell and isolated from atmosphere and the bulb by a high-vacuum Teflon valve (Kontes).

$\mathrm{Co}\left(\mathrm{dpgBF}_{2}\right)_{2}\left(\mathrm{CH}_{3} \mathrm{CN}\right)_{2}$ was synthesized under an inert atmosphere with degassed solvents according to a literature method, ${ }^{30}$ recrystallized in $\mathrm{CH}_{3} \mathrm{CN}$, and analyzed by cyclic voltammetry, absorption measurements, and elemental analysis. The hexafluorophosphate salts of $\left[\mathrm{Ru}(\mathrm{bpy})_{3}\right]^{2+}$ and methyl viologen $\left([\mathrm{MV}]^{2+}\right)$ were prepared from the corresponding chloride salts via salt metathesis with $\mathrm{NH}_{4} \mathrm{PF}_{6}$ in water $\left(0{ }^{\circ} \mathrm{C}\right)$ followed by filtration of the precipitated products from $\mathrm{NH}_{4} \mathrm{Cl}$.

$[\mathrm{Na}]\left[\mathrm{Co}\left(\mathrm{dpgBF}_{2}\right)_{2}\left(\mathrm{CH}_{3} \mathrm{CN}\right)\right]$ was prepared by reaction of $\mathrm{Co}(\mathrm{dpgBF})_{2}\left(\mathrm{CH}_{3} \mathrm{CN}\right)_{2}$ with excess sodium mercury amalgam $(0.5 \% \mathrm{Na})$ under vacuum in anhydrous acetonitrile, analogous to a literature preparation. ${ }^{5}$ The reduced species was transferred over a frit to a fused $2 \mathrm{~mm}$ quartz cuvette; the reduction process was monitored via UV-Vis absorption spectroscopy until the reduction was complete.

Methyl viologen cation radical $\left(\mathrm{MV}^{\bullet+}\right)$ was prepared in situ by the photolysis ( $\mathrm{Hg}$ lamp) of a degassed sample containing $10 \mu \mathrm{M}\left[\mathrm{Ru}(\mathrm{bpy})_{3}\right]\left[\mathrm{PF}_{6}\right]_{2}, 84 \mu \mathrm{M}[\mathrm{MV}]\left[\mathrm{PF}_{6}\right]_{2}, 0.1 \mathrm{M} \mathrm{NBu}_{4} \mathrm{PF}_{6}$, and $0.1 \mathrm{M}$ triethanolamine in $\mathrm{CH}_{3} \mathrm{CN}$ for $2 \mathrm{~h}$. The resulting absorption spectrum matched that from the literature. ${ }^{17}$

$\left[\mathrm{Ru}(\mathrm{bpy})_{3}\right]^{3+}$ was prepared in situ by oxidizing $\left[\mathrm{Ru}(\mathrm{bpy})_{3}\right]^{2+}$ with ammonium cerium nitrate $\left(\mathrm{Ce}^{\mathrm{IV}}\right)$ according to a literature method. ${ }^{12}$ 
$\mathrm{MeCo}\left(\mathrm{dpgBF}_{2}\right)_{2}(\mathrm{~L})\left(\mathrm{L}=\mathrm{H}_{2} \mathrm{O}, \mathrm{CH}_{3} \mathrm{CN}\right)$ was prepared by a method similar to that of Ram et al. ${ }^{31}$ for $\mathrm{MeCo}\left(\mathrm{dmgBF}_{2}\right)_{2}\left(\mathrm{H}_{2} \mathrm{O}\right) . \quad \mathrm{Co}\left(\mathrm{dpgBF}_{2}\right)_{2}\left(\mathrm{CH}_{3} \mathrm{CN}\right)_{2}(0.505 \mathrm{~g}, 0.724 \mathrm{mmol})$ was suspended under argon in $22 \mathrm{~mL}$ degassed $\mathrm{CH}_{3} \mathrm{OH}$ in a 2-neck $100 \mathrm{~mL}$ round-bottom flask equipped with a septum, vacuum adaptor, and stirbar. $\mathrm{NaOH}(0.091 \mathrm{~g}, 2.3 \mathrm{mmol})$ was added and the solution was stirred until the $\mathrm{NaOH}$ dissolved, followed by addition of pyridine $(0.058 \mathrm{~g}, 0.733 \mathrm{mmol})$. After cooling the suspension to $0{ }^{\circ} \mathrm{C}, \mathrm{NaBH}_{4}(0.046 \mathrm{~g}, 1.21$ mmol) was added, the reaction was stirred $15 \mathrm{~min}$, and the suspension turned a blue-gray color. Methyl trifluoromethanesulfonate $(1 \mathrm{~g}, 6.1 \mathrm{mmol})$ was added via syringe, and the suspension turned yellow-brown over $10 \mathrm{~min}$. The reaction was then exposed to the atmosphere, at which time $1 \mathrm{~mL}$ of pyridine and $40 \mathrm{~mL}$ of $\mathrm{H}_{2} \mathrm{O}$ were added $5 \mathrm{~min}$ apart. After 10 min of stirring, the product was filtered to yield a yellow-brown solid and washed with $3 \times 40 \mathrm{~mL} \mathrm{H}_{2} \mathrm{O}$. Then the product was collected, placed in a round-bottom flask, suspended in $30 \mathrm{~mL} 6 \mathrm{M} \mathrm{HClO}_{4}$, and stirred for $20 \mathrm{~min}$ to remove pyridine. The suspension was then filtered to yield a yellow-brown solid, and washed with 2 × $30 \mathrm{~mL} \mathrm{HClO}_{4}$ followed by copious amounts of $\mathrm{H}_{2} \mathrm{O}$ and $30 \mathrm{~mL}$ hexanes. The yellow-brown solid was collected (315 mg, 65\%). Vapor diffusion of ether into a saturated $\mathrm{CH}_{3} \mathrm{CN}$ solution of $\mathrm{MeCo}\left(\mathrm{dmgBF}_{2}\right)_{2}\left(\mathrm{CH}_{3} \mathrm{CN}\right)$ afforded a yellow powder. ${ }^{1} \mathrm{H} \mathrm{NMR}\left(300 \mathrm{MHz}, \mathrm{CD}_{3} \mathrm{CN}\right): \delta$ 7.28$7.48(\mathrm{~m}, 20 \mathrm{H}, \mathrm{Ph}), \delta 1.87\left(\mathrm{~s}, 3 \mathrm{H}, \mathrm{CH}_{3}\right) \mathrm{ppm}$. HRMS (FAB+), m/z calculated for $\mathrm{C}_{31} \mathrm{H}_{26} \mathrm{~B}_{2} \mathrm{~F}_{4} \mathrm{~N}_{5} \mathrm{O}_{4} \mathrm{Co}$ : 689.14. Found: $690.1559(\mathrm{M}+\mathrm{H}), 628.7951\left(\mathrm{M}-\mathrm{C}_{6} \mathrm{H}_{5}\right), 565.7864$ (M$\left.\mathrm{C}_{6} \mathrm{H}_{5}-\mathrm{BF}_{2}+\mathrm{H}\right), 513.9995\left(\mathrm{M}-\mathrm{C}_{6} \mathrm{H}_{5}-2 \mathrm{BF}_{2}\right), 485.9959$ (M- M- $\left.\mathrm{C}_{6} \mathrm{H}_{5}-2 \mathrm{BF}_{2}-2 \mathrm{CH}_{3}\right)$. Elemental analysis calculated for $\mathrm{C}_{29} \mathrm{H}_{25} \mathrm{~B}_{2} \mathrm{CoF}_{4} \mathrm{~N}_{4} \mathrm{O}_{5}$ : C, 52.29; H, 3.78; N, 8.41. Found: $\mathrm{C}, 52.62 ; \mathrm{H}$, 3.95; N, 8.31. 
Physical Methods

NMR spectra were recorded using a Varian Mercury 300 spectrometer. ${ }^{1} \mathrm{H}$ NMR chemical shifts were referenced to residual solvents as determined relative to $\mathrm{Me}_{4} \mathrm{Si}(\delta=0 \mathrm{ppm})$. High resolution mass spectra (HRMS) were obtained at the California Institute of Technology Mass Spectrometry Facility. UV-visible absorption measurements were carried out using a Hewlett Packard 8452 spectrophotometer in 0.2 or $1 \mathrm{~cm}$ pathlength quartz cuvettes.

Time-resolved spectroscopic measurements were carried out at the Beckman Institute Laser Resource Center. Laser excitation was provided by $8 \mathrm{~ns}$ pulses from a $10 \mathrm{~Hz}$ Q-switched Nd:YAG laser (Spectra-Physics Quanta-Ray PRO-Series). The third harmonic was either used directly or to pump an optical parametric oscillator (OPO, Spectra-Physics QuantaRay MOPO-700, tunable in the visible region).

Probe light for transient absorption kinetics measurements was provided by a $75 \mathrm{~W}$ arc lamp (PTI Model A 1010) that could be operated in continuous wave or pulsed modes. Timing between the laser and the probe light was controlled by a digital delay generator (EG\&G 9650). After passing through the sample collinearly with the laser beam, scattered excitation light was rejected by suitable long pass and short pass filters, and probe wavelengths were selected for detection by a double monochromator (Instruments SA DH10) with $1 \mathrm{~mm}$ slits. Transmitted light was detected with a photomultiplier tube (PMT, Hamamatsu R928). The PMT current was amplified and recorded with a transient digitizer (LeCroy 9354A or Tektronix DSA 602). Absorption data were averaged over at least 500 laser pulses. The data were converted to units of $\Delta \mathrm{OD}\left(\Delta \mathrm{OD}=-\log _{10}\left(\mathrm{I} / \mathrm{I}_{0}\right)\right.$, where $\mathrm{I}$ is the time-resolved probe-light intensity with laser excitation, and $\mathrm{I}_{0}$ is the intensity without excitation). Samples measured on the microsecond timescale or faster were stirred and measured using a laser repetition rate of $10 \mathrm{~Hz}$, while samples measured on a millisecond 
timescale were excited with a single shutter-released laser pulse, stirred for $1 \mathrm{~s}$ after collecting data, then allowed to sit until the solution settled ( $2 \mathrm{~s})$ before the next laser pulse.

Probe light for transient absorption spectra was provided by white light flash lamp sources with either nanosecond or microsecond durations. Probe light was transported via a fiber optic and split by a partial reflector. Approximately $70 \%$ of the probe light was passed through the sample, with the remainder directed around the sample as a reference beam. Sample excitation $\left(\lambda_{\mathrm{ex}}=355 \mathrm{~nm}\right)$ by the laser beam was collinear with the probe light. Light intensity was read by two photodiode arrays (Ocean Optics S1024DW Deep Well Spectrometer), with scattered excitation light rejected by a $370 \mathrm{~nm}$ long pass filter. The timing synchronization of the laser fire, flashlamp fire, and photodiode array readout was controlled by a series of timing circuits triggered by either a Q-switch advance logic pulse for nanosecond (or a laser lamp sync pulse for microsecond) lamp measurements. The photodiode readout was interfaced with a PC via a National Instruments multifunction input/output card. Measurements were made with and without excitation, corrected for background light, and corrected for fluorescence when necessary. Difference spectra were averaged over approximately 500 shots.

All instruments and electronics in these systems were controlled by software written in LabVIEW (National Instruments). Data manipulation was performed with MATLAB R2008a (Mathworks, Inc.) and graphed with Igor Pro 5.01 (Wavemetrics).

\section{Data Analysis}

Global analysis and kinetics simulations were performed in MATLAB, the latter using the ordinary differential equation solver ode23s (Rosenbrock method) and an m-file with the differential equations (Appendix C). $\left[\mathrm{Ru}(\mathrm{bpy})_{3}\right]^{2+*}$ luminesces weakly at the wavelength the 
kinetics traces were measured. Because of the finite amplifier response for the longer timescales, the first few microseconds of data were obscured by this emission process.

\section{References}

1. Hu, X. L.; Cossairt, B. M.; Brunschwig, B. S.; Lewis, N. S.; Peters, J. C. Chem. Commun. $2005,4723$.

2. Appel, A. M.; DuBois, D. L.; Rakowski DuBois, M. J. Am. Chem. Soc. 2005, 127, 12717.

3. Wilson, A. D.; Newell, R. H.; McNevin, M. J.; Muckerman, J. T.; Rakowski DuBois, M.; DuBois, D. L. J. Am. Chem. Soc. 2005, 128, 358.

4. Razavet, M.; Artero, V.; Fontecave, M. Inorg. Chem. 2005, 44, 4786.

5. Hu, X.; Brunschwig, B. S.; Peters, J. C. J. Am. Chem. Soc. 2007, 129, 8988.

6. Baffert, C.; Artero, V.; Fontecave, M. Inorg. Chem. 2007, 46, 1817.

7. Connolly, P.; Espenson, J. H. Inorg. Chem. 1986, 25, 2684.

8. Ogata, T.; Yanagida, S.; Brunschwig, B. S.; Fujita, E. J. Am. Chem. Soc. 1995, 117, 6708.

9. Bjerrum, M. J.; Casimiro, D. R.; Chang, I. J.; Dibilio, A. J.; Gray, H. B.; Hill, M. G.; Langen, R.; Mines, G. A.; Skov, L. K.; Winkler, J. R.; Wuttke, D. S. J. Bioenerg. Biomembr. 1995, 27, 295.

10. Chiorboli, C.; Indelli, M. T.; Rampi Scandola, M. A.; Scandola, F. J. Phys. Chem. 1988, $92,156$.

11. Clark, C. D.; Hoffman, M. Z. J. Phys. Chem. 1996, 100, 7526.

12. Bryant, G. M.; Fergusso, J. Aust. J. Chem. 1971, 24, 275.

13. Marcus, R. A.; Sutin, N. Biochim. Biophys. Acta 1985, 811, 265.

14. Young, R. C.; Keene, F. R.; Meyer, T. J. J. Am. Chem. Soc. 1977, 99, 2468.

15. Chan, M.-S.; Wahl, A. C. J. Phys. Chem. 1978, 82, 2542.

16. Rieger, A. L.; Rieger, P. H. J. Phys. Chem. 1984, 88, 5845. 
17. Kosower, E. M.; Cotter, J. L. J. Am. Chem. Soc. 1964, 86, 5524.

18. Chen, P.; Curry, M.; Meyer, T. J. Inorg. Chem. 1989, 28, 2271.

19. Felton, G. A. N.; Glass, R. S.; Lichtenberger, D. L.; Evans, D. H. Inorg. Chem. 2006, 45, 9181.

20. Wangila, G. W.; Jordan, R. B. Inorg. Chim. Acta 2005, 358, 2804.

21. Endicott, J. F.; Durham, B.; Glick, M. D.; Anderson, T. J.; Kuszaj, J. M.; Schmonsees, W. G.; Balakrishnan, K. P. J. Am. Chem. Soc. 1981, 103, 1431.

22. Szalda, D. J.; Creutz, C.; Mahajan, D.; Sutin, N. Inorg. Chem. 1983, 22, 2372.

23. Creutz, C.; Chou, M. H.; Fujita, E.; Szalda, D. J. Coord. Chem. Rev. 2005, 249, 375.

24. Artero, V.; Fontecave, M. Coord. Chem. Rev. 2005, 249, 1518.

25. Berning, D. E.; Noll, B. C.; DuBois, D. L. J. Am. Chem. Soc. 1999, 121, 11432.

26. Bhugun, I.; Lexa, D.; Savéant, J.-M. J. Am. Chem. Soc. 1996, 118, 3982.

27. Chao, T.-H.; Espenson, J. H. J. Am. Chem. Soc. 1978, 100, 129.

28. Kellett, R. M.; Spiro, T. G. Inorg. Chem. 1985, 24, 2373.

29. Pangborn, A. B.; Giardello, M. A.; Grubbs, R. H.; Rosen, R. K.; Timmers, F. J. Organometallics 1996, 15, 1518.

30. Tovrog, B. S.; Kitko, D. J.; Drago, R. S. J. Am. Chem. Soc. 1976, 98, 5144.

31. Ram, M. S.; Riordan, C. G.; Yap, G. P. A.; LiableSands, L.; Rheingold, A. L.; Marchaj, A.; Norton, J. R. J. Am. Chem. Soc. 1997, 119, 1648. 
Chapter 3

\section{MECHANISM OF $\mathrm{H}_{2}$ EVOLUTION FROM A PHOTOGENERATED HYDRIDOCOBALOXIME}

Portions of this work are excerpted with permission from:

Dempsey, J. L.; Winkler, J. R.; Gray, H. B. J. Am. Chem. Soc. 2010, 132, 16774-16776.

Unpublished Material Copyright 2010 American Chemical Society 


\section{Introduction}

The mechanisms of $\mathrm{H}_{2}$ production catalyzed by molecular catalysts, like difluoroboryl bridged $\mathrm{Co}^{\mathrm{II}}$-diglyoxime complexes, are of great interest, as these catalysts are key elements in proposed solar-driven water splitting devices. ${ }^{1}$ Among the most promising catalysts are those that rapidly evolve $\mathrm{H}_{2}$ from acidic solutions, but in such cases it is difficult to observe catalytic intermediates by traditional spectroscopic methods. Several reaction mechanisms have been proposed that begin with protonation of a $\mathrm{Co}^{\mathrm{I}}$ complex to form $\mathrm{Co}^{\mathrm{III}} \mathrm{H} ; \mathrm{H}_{2}$ evolution can occur via protonation of $\mathrm{Co}^{\mathrm{III}} \mathrm{H}$ or upon bimolecular combination of two $\mathrm{Co}^{\mathrm{III}} \mathrm{H}$ species. ${ }^{1}$ Alternatively, $\mathrm{Co}^{\mathrm{III}} \mathrm{H}$ can be reduced further to form $\mathrm{Co}^{\mathrm{II}} \mathrm{H}$, which can react via similar heterolytic or homolytic routes. As discussed in Chapter 2, we have utilized laser flash-quench techniques to trigger the reduction of a $\mathrm{Co}^{\mathrm{II}}$-diglyoxime species to $\mathrm{Co}^{\mathrm{I}}{ }^{2}$ In these experiments, time-resolved spectroscopy was used to detect the photochemically generated reduced species and monitor its reactivity. Analysis of these electron transfer reactions provided estimates of the barriers associated with the elementary steps in the proposed mechanistic cycles. Although reactivity through a homolytic route is favored, we were not able to obtain the kinetics of protonation and subsequent $\mathrm{H}_{2}$ evolution steps with these transient techniques, as the strong acids needed for protonation caused catalyst degradation.

This chapter describes an alternative technique based on excited-state proton transfer that has allowed us to trigger protonation of a $\mathrm{Co}^{\mathrm{I}}$-diglyoxime species and investigate the kinetics of catalytic $\mathrm{H}_{2}$ evolution. Our approach employs photoacids that produce powerful proton donors upon excitation..$^{3-6}$ Although these $\mathrm{p} K_{\mathrm{a}}$ jumps often are confined to the

nanosecond lifetimes of singlet excited states, substitution with bromine facilitates intersystem crossing to generate high yields of long-lived triplet excited states, enabling 
bimolecular proton transfer reactions. ${ }^{7-8} \mathrm{~A}$ case in point is the report of excited-state proton transfer from the triplet excited state of 6-bromo-2-naphthol $\left({ }^{\mathrm{Br}} \mathrm{NaphOH}\right)$ to triethylamine in acetonitrile solution. ${ }^{9}$ Utilizing this new phototriggering technique, we have observed excited-state proton transfer from ${ }^{\mathrm{Br}} \mathrm{NaphOH}$ to a reduced cobaloxime, $[\mathrm{Na}]\left[\mathrm{Co}^{\mathrm{I}}\left(\mathrm{dmgBF}_{2}\right)_{2}\left(\mathrm{CH}_{3} \mathrm{CN}\right)\right] \quad\left([\mathrm{Na}]\left[\mathrm{Co}^{\mathrm{I}}\right], \quad[\mathrm{Na}]\left[\mathbf{1}^{-}\right]\right), \quad$ forming a hydride, $\mathrm{HCo}^{\mathrm{III}}\left(\mathrm{dmgBF}_{2}\right)_{2}\left(\mathrm{CH}_{3} \mathrm{CN}\right)\left(\left[\mathbf{C o}^{\mathrm{III}} \mathbf{H}\right]\right)$, which subsequently reacts to produce $\mathrm{H}_{2}$ (Scheme $3.1)$.

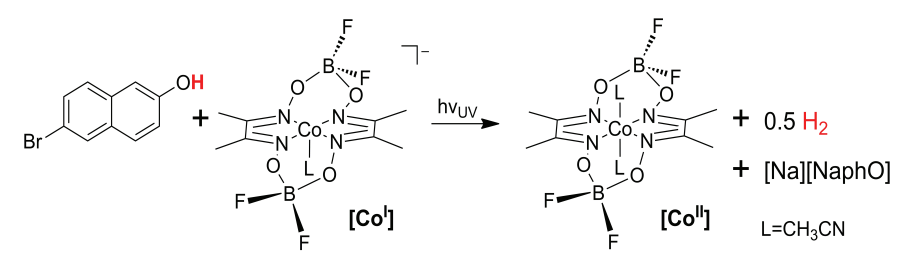

Scheme 3.1

\section{Results and Discussion}

The $\mathrm{p} K_{\mathrm{a}}$ of ${ }^{\mathrm{B}} \mathrm{NaphOH}$ in DMSO has been reported as $16.2 .{ }^{10}$ The $\mathrm{pK}_{\mathrm{a}}$ of ${ }^{\mathrm{Br}} \mathrm{NaphOH}$ in $\mathrm{CH}_{3} \mathrm{CN}$ was estimated from the DMSO value by the method of Koppel and coworkers, shown in Equation 3.1, to give a $\mathrm{p} K_{\mathrm{a}}$ of $26.1 .^{11}$

$\mathrm{p} K_{\mathrm{a}}\left(\mathrm{CH}_{3} \mathrm{CN}\right)=11.80+0.884\left(\mathrm{p} K_{\mathrm{a}}(\mathrm{DMSO})\right)=26.1 \quad$ Equation 3.1

The Förster cycle ${ }^{3,12}$ was used to estimate the $\mathrm{p} K_{\mathrm{a}}^{*}$ of photoexcited ${ }^{\mathrm{B}} \mathrm{NaphOH}$ (equation $3.2)$.

$\mathrm{p} K_{\mathrm{a}}-\mathrm{p} K_{\mathrm{a}}^{*}=\left(\mathrm{E}_{\mathrm{HA}}-\mathrm{E}_{\mathrm{A}-}\right) /\left(2.3 \mathrm{k}_{\mathrm{B}} \mathrm{T}\right)$

Equation 3.2 
For ${ }^{* 1} \mathrm{p} K_{\mathrm{a}}, \mathrm{E}_{\mathrm{HA}}$ and $\mathrm{E}_{\mathrm{A} \text { - }}$ are the difference between $\mathrm{S}_{0}$ and $\mathrm{S}_{1}\left(\mathrm{E}_{0,0}\right)$ in wavenumbers. For ${ }^{*} \mathrm{p} K_{\mathrm{a}}, \mathrm{E}_{0,0}$ is estimated from the intersection of normalized absorption and fluorescence spectra of the naphthol and naphtholate, and converted to wavenumbers (Figure 3.1, Figure

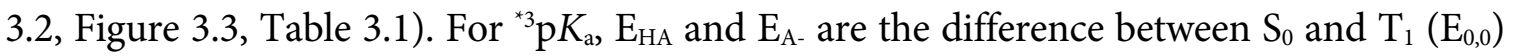
in wavenumbers. $E_{0,0}$ is estimated from the highest energy of the structured emission observed in the low temperature phosphorescence spectra (Figure 3.4, Figure 3.5, Table 3.2). As estimated from this method, the singlet and triplet excited states have $\mathrm{p} K_{\mathrm{a}}$ values of approximately 13.7 and 14.6 (Table 3.3), ${ }^{3,12}$ respectively, which suggested that while the photoacid would not react with $[\mathrm{Na}]\left[\mathrm{Co}^{\mathrm{I}}\right]$ in its ground state, excited-state proton transfer should be favorable, as $\left[\mathbf{C o}^{\mathrm{I}}\right]$ is readily protonated by trifluoroacetic acid $\left(\mathrm{p} K_{\mathrm{a}}=12.7\right){ }^{13}$

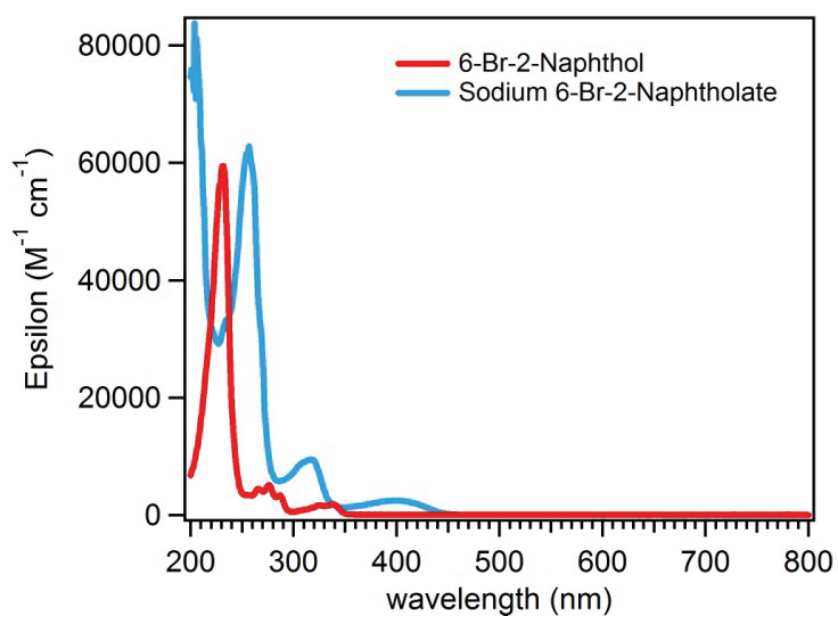

Figure 3.1 Absorption Spectra of 6-bromo-2-naphthol (red) and sodium 6-bromo-2-naphtholate (blue) in acetonitrile. 


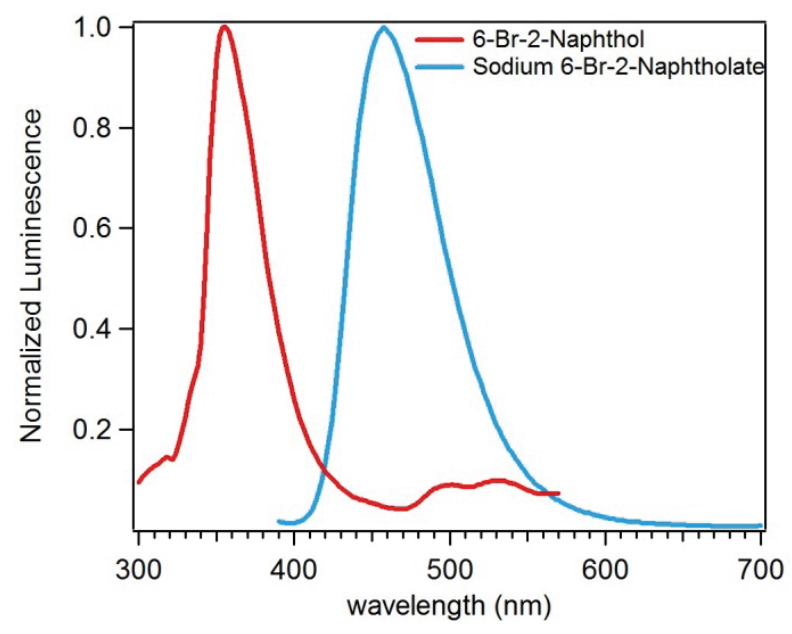

Figure 3.2 Normalized luminescence spectra of 6-bromo-2naphthol (red) and sodium 6-bromo-2-naphtholate (blue), room temperature, in acetonitrile solution. $\lambda_{\text {ex }}$ (6-bromo-2-naphthol) $=$ $290 \mathrm{~nm}, \lambda_{\text {ex }}$ (sodium 6-bromo-2-naphtholate) $=380 \mathrm{~nm}$. Weak phosphorescence at room temperature is observed for 6-bromo-2naphthol but not sodium 6-bromo-2-naphtholate.

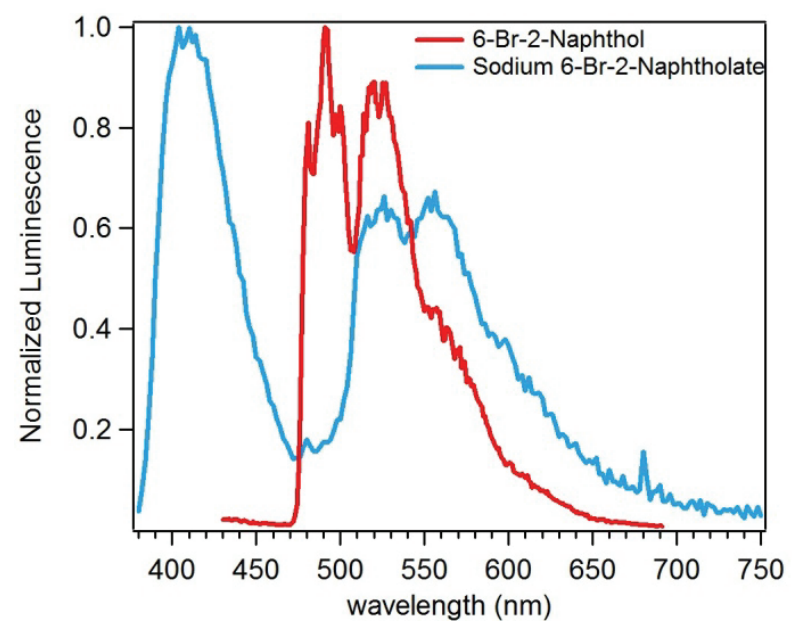

Figure 3.3 Normalized luminescence spectra of 6-bromo-2naphthol (red) and sodium 6-bromo-2-naphtholate (blue), 77K, in butyronitrile glass. $\lambda_{\mathrm{ex}}$ (6-bromo-2-naphthol) $=340 \mathrm{~nm}, \lambda_{\mathrm{ex}}$ (sodium 6-bromo-2-naphtholate) $=340 \mathrm{~nm}$. Both fluorescence and phosphorescence were measured for sodium 6-bromo-2naphtholate, but the fluorescence of 6-bromo-2-naphthol was not measured at $77 \mathrm{~K}$ due to experimental limitation. 


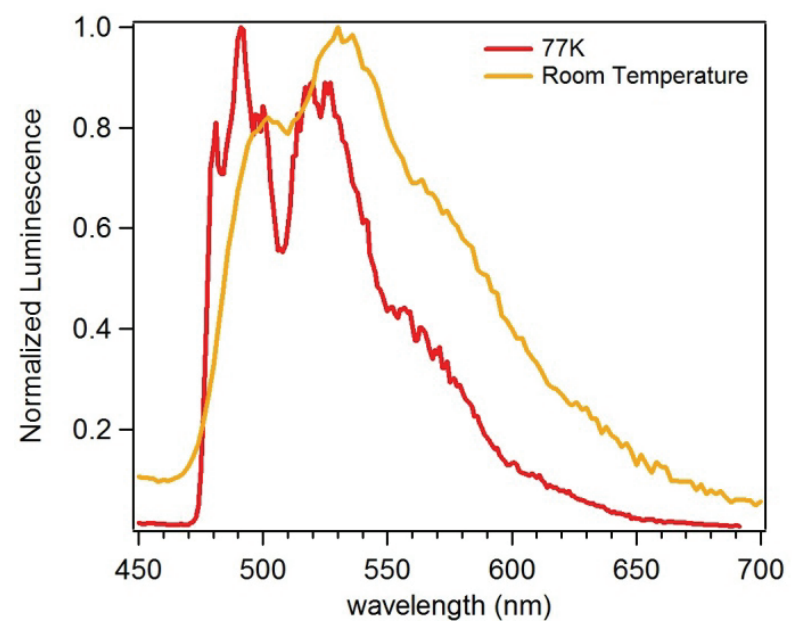

Figure 3.4 Normalized phosphorescence spectra of 6-bromo-2naphthol at $77 \mathrm{~K}$ (red, in butyronitrile glass) and at room temperature (orange, in acetonitrile solution). $\lambda_{\text {ex }}(77 \mathrm{~K})=340 \mathrm{~nm}$, $\lambda_{\text {ex }}($ room temperature $)=290 \mathrm{~nm}$.

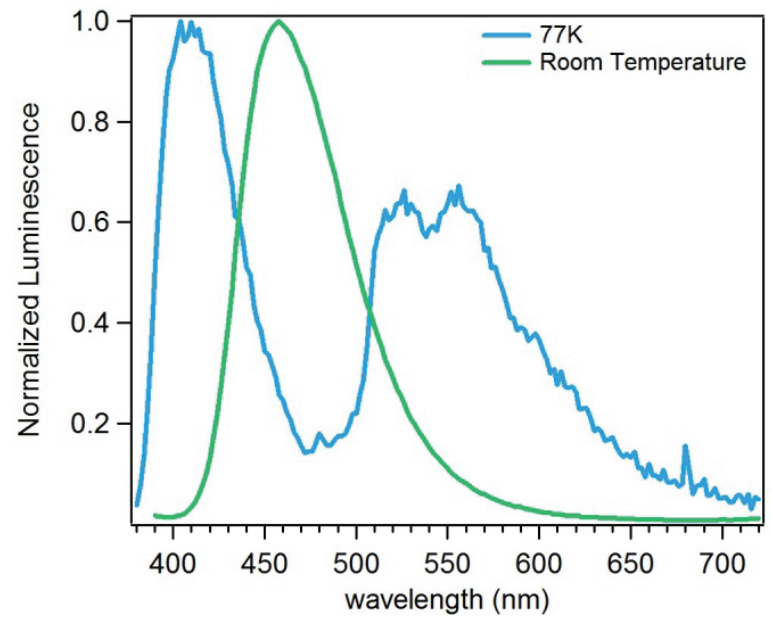

Figure 3.5 Normalized luminescence spectra of sodium 6-bromo2-naphtholate at $77 \mathrm{~K}$ (blue, in butyronitrile glass) and at room temperature (green, in acetonitrile solution). $\lambda_{\text {ex }}(77 \mathrm{~K})=340 \mathrm{~nm}$, $\lambda_{\text {ex }}($ room temperature $)=380 \mathrm{~nm}$. 
Table 3.1 Absorption and fluorescence maxima in $\mathrm{CH}_{3} \mathrm{CN}$ at room temperature.

\begin{tabular}{llll}
\hline & Abs. Max $\left(\mathrm{cm}^{-1}\right)$ & Fluorescence Max $\left(\mathrm{cm}^{-1}\right)$ & $\mathbf{E}_{0,0}\left(\mathbf{c m}^{-1}\right)$ \\
\hline${ }^{\mathrm{Br}} \mathrm{NaphOH}$ & 29,586 & 28,090 & 29,070 \\
{$\left[\mathrm{Na}^{+}\right]\left[{ }^{\text {Br}} \mathrm{NaphO}^{-}\right]$} & 24,876 & 21,834 & 23,148 \\
$\mathrm{E}_{\mathrm{HA}}-\mathrm{E}_{\mathrm{A}-}$ & 4,710 & 6,256 & 5,922 \\
\hline
\end{tabular}

Table 3.2 Phosphorescence data in butyronitrile at 77K.

\begin{tabular}{ll}
\hline & $\mathbf{E}_{0,0}\left(\mathrm{~cm}^{-1}\right)$ \\
\hline${ }^{\mathrm{Br}} \mathrm{NaphOH}$ & 20,790 \\
{$\left[\mathrm{Na}^{+}\right]\left[{ }^{\mathrm{Br}} \mathrm{NaphO}^{-}\right]$} & 19,379 \\
$\mathrm{E}_{\mathrm{HA}}-\mathrm{E}_{\mathrm{A}-}$ & 1,411 \\
\hline
\end{tabular}

Table 3.3 Excited state $\mathrm{p} K_{\mathrm{a}}$ values in $\mathrm{CH}_{3} \mathrm{CN}$ estimated from the Förster cycle.

\begin{tabular}{llll}
\hline Excited State & $\mathbf{E}_{\mathrm{HA}-\mathbf{E}_{\mathrm{A}-} \text { calculated from }}$ & Temperature $(\mathbf{K})$ & $\mathbf{p} \boldsymbol{K}_{\mathrm{a}}^{*}$ \\
\hline Singlet & Fluorescence Maxima & 298 & 13.0 \\
Singlet & Fluorescence $\mathrm{E}_{0,0}$ & 298 & 13.7 \\
Triplet & Phosphorescence $\mathrm{E}_{0,0}$ & 77 & 14.6 \\
\hline
\end{tabular}

To test the reactivity of ${ }^{3 *}[\mathrm{Br} N a p h O H]$ with $\left[\mathrm{Co}^{\mathrm{I}}\right]$, an acetonitrile solution containing 672 $\mu \mathrm{M}{ }^{\mathrm{Br}} \mathrm{NaphOH}, 167 \mu \mathrm{M}$ [Na][Co $\left.{ }^{\mathrm{I}}\right]$, and $100 \mathrm{mM}\left[\mathrm{NBu}_{4}\right]\left[\mathrm{PF}_{6}\right]$ was irradiated with $266 \mathrm{~nm}$ light in a $1 \mathrm{~cm}$ quartz cuvette. Absorption spectra measured at intervals indicated clean and quantitative conversion of $\left[\mathbf{C o}^{\mathrm{I}}\right]$ to $\left[\mathbf{C o}^{\mathrm{II}}\right]$ (Figure 3.6, Figure 3.7), consistent with the overall reaction shown in Scheme 3.1. Global analysis was performed to determine the concentrations of $\left[\mathrm{Co}^{\mathrm{I}}\left(\mathrm{dmgBF}_{2}\right)_{2}\left(\mathrm{CH}_{3} \mathrm{CN}\right)\right]^{-}, \mathrm{Co}^{\mathrm{II}}\left(\mathrm{dmgBF}_{2}\right)_{2}\left(\mathrm{CH}_{3} \mathrm{CN}\right)_{2}$, and $[\mathrm{Na}]\left[{ }^{\mathrm{Br}} \mathrm{NaphO}\right]$ at each time interval ( ${ }^{\mathrm{Br}} \mathrm{NaphOH}$ does not absorb in the region of interest). Interestingly, the absorption of $[\mathrm{Na}]\left[{ }^{\mathrm{Br}} \mathrm{NaphO}\right]$ made up a negligible component of the spectra, while the 
sum of $\left[\mathrm{Co}^{2+}\right]$ and $\left[\mathrm{Co}^{+}\right]$was consistently $\sim 170 \mu \mathrm{M}$ (Table 3.5), suggesting a reactive pathway for the naphtholate decomposition, as observed by Pretali et al. ${ }^{9}$

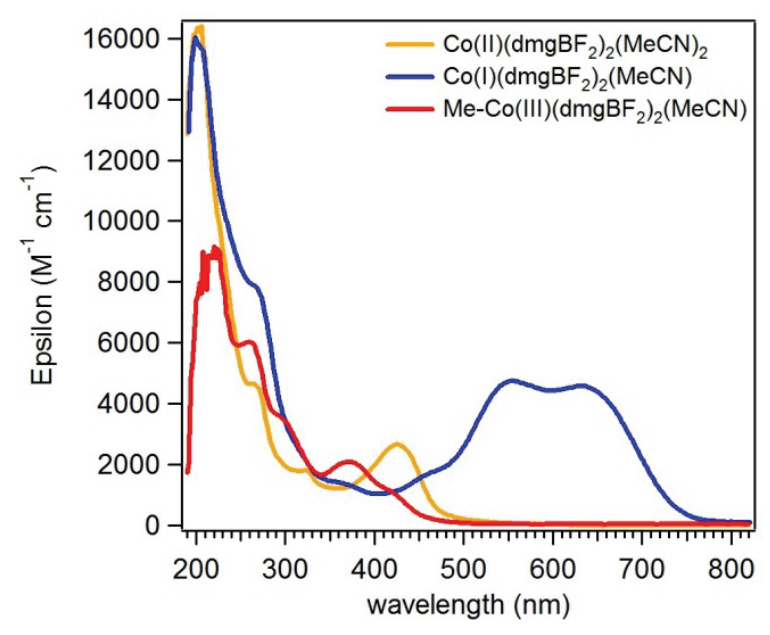

Figure 3.6 Absorption spectra of $\mathrm{Co}(\mathrm{II})\left(\mathrm{dmgBF}_{2}\right)_{2}\left(\mathrm{CH}_{3} \mathrm{CN}\right)_{2}$, $\left.[\mathrm{Na}]\left[\mathrm{Co}(\mathrm{I})\left(\mathrm{dmgBF}_{2}\right)_{2}\left(\mathrm{CH}_{3} \mathrm{CN}\right)\right], \quad \mathrm{MeCo}(\mathrm{III})\left(\mathrm{dmgBF}_{2}\right)_{2}\left(\mathrm{CH}_{3} \mathrm{CN}\right)\right]$ in acetonitrile. Epsilon values available in Table 3.4.

Table 3.4 Absorption characterization.

\begin{tabular}{ll}
\hline Sample & UV-Vis Absorption/nm $\left(\right.$ Epsilon/( $\left.\left.\mathbf{M}^{-1} \mathbf{s}^{-1}\right)\right)$ \\
\hline 6-bromo-2-naphthol & $232(59,450), 276(5,111), 339(1,851)$ \\
Sodium 6-bromo-2-naphtholate & $257(62,785), 316(9,484), 402(2,489)$ \\
$\mathrm{Co}(\mathrm{II})(\mathrm{dmgBF})_{2}\left(\mathrm{CH}_{3} \mathrm{CN}\right)_{2}$ & $260(4,645), 322(1,810), 424(2,660)$ \\
{$[\mathrm{Na}]\left[\mathrm{Co}(\mathrm{I})\left(\mathrm{dmgBF}_{2}\right)_{2}\left(\mathrm{CH}_{3} \mathrm{CN}\right)\right]$} & $264(7,905), 553(4,712), 628(4,546)$ \\
$\mathrm{Me}-\mathrm{Co}(\mathrm{III})\left(\mathrm{dmgBF}_{2}\right)_{2}\left(\mathrm{CH}_{3} \mathrm{CN}\right)$ & $258(6,042), 370(2,090)$ \\
\hline
\end{tabular}




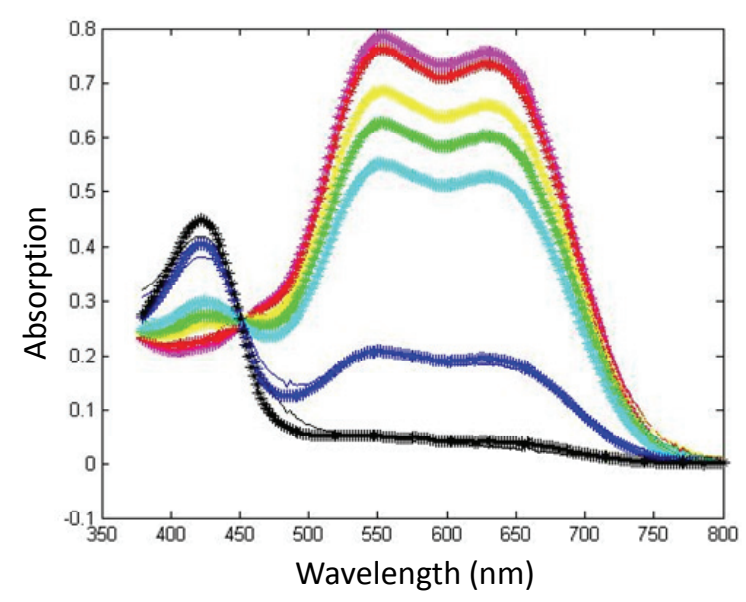

Figure 3.7 Absorption spectra (solid lines) and corresponding global fits (starred lines) monitored at different time points during photolysis. Sample initially contained $672 \mu \mathrm{M}$ 6-bromo-2naphthol, $167 \mu \mathrm{M}[\mathrm{Na}]\left[\mathrm{Co}\left(\mathrm{dmgBF}_{2}\right)_{2}\left(\mathrm{CH}_{3} \mathrm{CN}\right)\right]$, and $100 \mathrm{mM}$ $\left[\mathrm{NBu}_{4}\right]\left[\mathrm{PF}_{6}\right]$ in acetonitrile in a $1 \mathrm{~cm}$ pathlength cuvette. (Sample 1: pink, sample 2: red, sample 3: yellow, sample 4: green, sample 5: cyan, sample 6: blue, sample 7, black)

Table 3.5 Sample components for each absorption measurement in Figure 3.7, obtained from global analysis.

\begin{tabular}{rrrr}
\hline Sample & \multicolumn{1}{c}{$\left[\mathbf{C o}^{\mathrm{I}}\right]$} & {$\left[\mathrm{Co}^{\mathrm{II}}\right]$} & [6-Br-2-naphtolate $]$ \\
\hline $\mathbf{1}$ & $1.66 \times 10^{-4}$ & $3.30 \times 10^{-6}$ & $1.88 \times 10^{-5}$ \\
$\mathbf{2}$ & $1.61 \times 10^{-4}$ & $8.80 \times 10^{-6}$ & $1.98 \times 10^{-5}$ \\
$\mathbf{3}$ & $1.45 \times 10^{-4}$ & $2.68 \times 10^{-5}$ & $1.99 \times 10^{-5}$ \\
$\mathbf{4}$ & $1.32 \times 10^{-4}$ & $3.71 \times 10^{-5}$ & $2.09 \times 10^{-5}$ \\
$\mathbf{5}$ & $1.16 \times 10^{-4}$ & $5.20 \times 10^{-5}$ & $2.22 \times 10^{-5}$ \\
$\mathbf{6}$ & $4.22 \times 10^{-5}$ & $1.19 \times 10^{-4}$ & $2.52 \times 10^{-5}$ \\
$\mathbf{7}$ & $8.30 \times 10^{-6}$ & $1.49 \times 10^{-4}$ & $2.61 \times 10^{-5}$ \\
\hline
\end{tabular}

To confirm the production of $\mathrm{H}_{2}$, bulk photolysis of a $40 \mathrm{~mL}$ stirring sample containing ca. $500 \mu \mathrm{M}[\mathrm{Na}]\left[\mathrm{Co}^{\mathrm{I}}\right], 5 \mathrm{mM}{ }^{\mathrm{Br}} \mathrm{NaphOH}$, and $50 \mathrm{mM}\left[\mathrm{NBu}_{4}\right]\left[\mathrm{PF}_{6}\right]$ was performed in a custom $61 \mathrm{~mL}$ round-bottom flask fitted with a 1 inch diameter quartz window and a Teflon valve. After $\left[\mathrm{Co}^{\mathrm{I}}\right]$ was converted to $\left[\mathrm{Co}^{\mathrm{II}}\right]$, monitored qualitatively via solution color change (Figure 3.8), the composition of the headspace gas was determined via gas chromatography. 
Quantifiable concentrations of $\mathrm{H}_{2}$ were observed, with yields up to 64\% based on the initial concentration of $[\mathrm{Na}]\left[\mathrm{Co}^{\mathrm{I}}\right]$ (Table 3.6). Incomplete conversion is attributed to the GC detection limit as well as decomposition associated with the harsh conditions needed for photolysis of a concentrated solution.

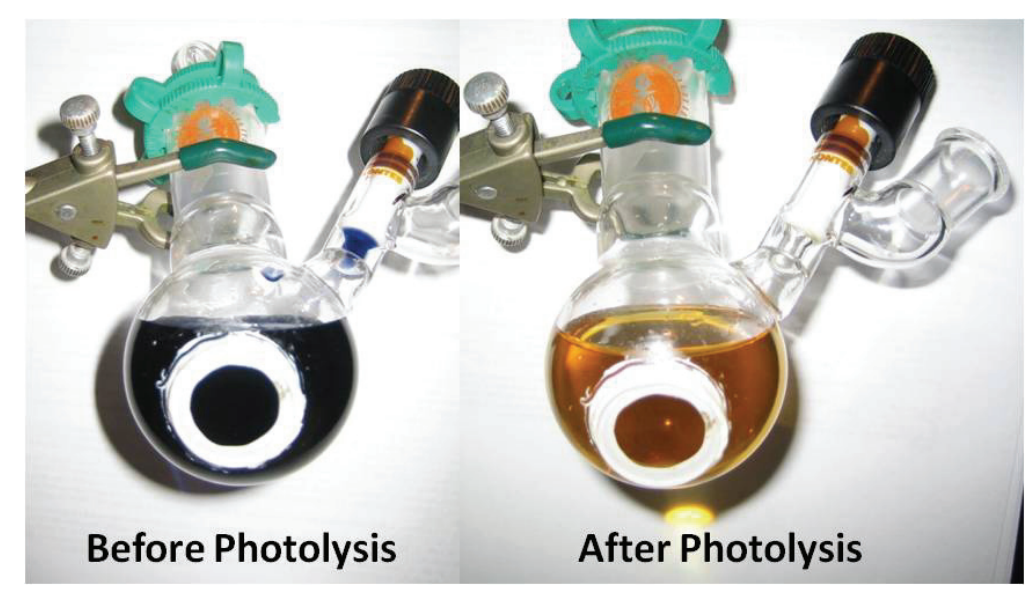

Figure 3.8 Photographs of a bulk photolysis sample before and after photolysis at $266 \mathrm{~nm}$. The sample was photolyzed through the round quartz window, and the headspace was sampled through the Teflon valve.

Table 3.6 Hydrogen evolution yields from bulk photolysis experiments.

\begin{tabular}{llll}
\hline Run & {$\left[\mathrm{Co}\left(\mathrm{dmgBF}_{2}\right)_{2}\left(\mathrm{CH}_{3} \mathrm{CN}\right)^{-}\right](\mu \mathrm{M})$} & ${\text { \% } \mathrm{H}_{2} \text { in Headspace }}$ & \% Yield \\
\hline 1 & 510 & 0.65 & 64 \\
2 & 446 & 0.39 & 38 \\
\hline
\end{tabular}

With these results in hand, time-resolved measurements were carried out to monitor the proton transfer kinetics. Upon pulsed-laser excitation of ${ }^{\mathrm{Br}} \mathrm{NaphOH}$ in the presence of $[\mathrm{Na}]\left[\mathrm{Co}^{\mathrm{I}}\right]\left(\lambda_{\text {ex }}=266 \mathrm{~nm}, 3 \mathrm{~mJ} /\right.$ pulse $)$, we observed accelerated decay of the ${ }^{3^{*}}\left[{ }^{\mathrm{Br}} \mathrm{NaphOH}\right]$ transient absorption $(460 \mathrm{~nm})$ attributable to excited-state proton transfer to form $\left(\left[\mathbf{C o}{ }^{\mathrm{III}} \mathbf{H}\right]\right)$ and $[\mathrm{Na}]\left[{ }^{\mathrm{Br}} \mathrm{NaphO}\right]$. Stern-Volmer quenching analysis yields a protonation rate 
constant $\left(k_{\mathrm{p}}\right)$ of $4.7 \times 10^{9} \mathrm{M}^{-1} \mathrm{~s}^{-1}$ (Figure 3.9). The consumption of [ $\left.\mathrm{Co}^{\mathrm{I}}\right]$ upon protonation was monitored via single wavelength transient absorption at $630 \mathrm{~nm}$ (Figure 3.10). Two kinetics processes were observed, a fast reaction on the microsecond timescale followed by a slower reaction occurring over milliseconds (vide infra). The faster process is attributed to protonation of $[\mathbf{C o}]$ to form $\left[\mathbf{C o}^{\mathrm{III}} \mathbf{H}\right]$, a species expected to have weak visible spectral features analogous to those of the yellow isolable $\mathrm{Co}^{\mathrm{III}} \mathrm{CH}_{3}$ complex, $\mathrm{MeCo}^{\mathrm{III}}\left(\mathrm{dmgBF}_{2}\right)_{2}\left(\mathrm{CH}_{3} \mathrm{CN}\right)$ (Figure 3.6). ${ }^{14}$ The fast kinetics phase best fit a single exponential decay plus a linear component to account for the slower decay process (Figure 3.10, top inset). The first-order rate constant for decay is linearly dependent on the concentration of $\left[\mathrm{Co}^{\mathrm{I}}\right]$ under these pseudo-first-order conditions (cobaloxime in excess), giving a second-order rate constant for protonation $\left(k_{\mathrm{p}}\right)$ of $3.5 \times 10^{9} \mathrm{M}^{-1} \mathrm{~s}^{-1}$ in agreement with the Stern-Volmer quenching analysis (Figure 3.11). 


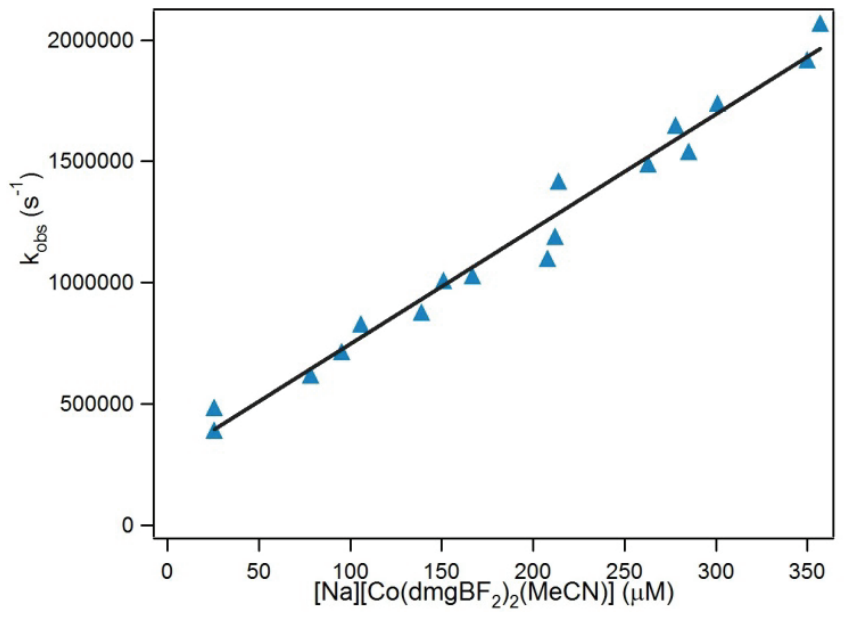

Figure 3.9 First-order rate constants determined from excitedstate quenching of 6-bromo-2-naphthol $\left(\lambda_{\text {ex }}=266 \mathrm{~nm}, \lambda_{\text {obs }}=460\right.$ $\mathrm{nm})$ in the presence of $\left[\mathrm{Co}\left(\mathrm{dmgBF}_{2}\right)_{2}\left(\mathrm{CH}_{3} \mathrm{CN}\right)\right]^{-}$. In the absence of a proton accepting base, the transient absorption decay of ${ }^{*}[6$ Br-2-Naphthol] follows mainly biexponential kinetics, with rate constants of $6.4 \times 10^{4} \mathrm{~s}^{-1}$ and $4.0 \times 10^{5} \mathrm{~s}^{-1}$; this data point is not included in the Stern-Volmer plot above. We found linear relationships between first-order rate constants and cobaloxime concentrations, allowing determination of a second-order rate constant for protonation, $k_{\mathrm{obs}}=k_{0}+k_{\mathrm{p}}\left[\mathrm{Co}\left(\mathrm{dmgBF}_{2}\right)_{2}\left(\mathrm{CH}_{3} \mathrm{CN}\right)\right]^{-}$, gives $k_{0}=2.7 \times 10^{5} \mathrm{~s}^{-1}, k_{\mathrm{p}}=4.7 \times 10^{9} \mathrm{M}^{-1} \mathrm{~s}^{-1}$. 


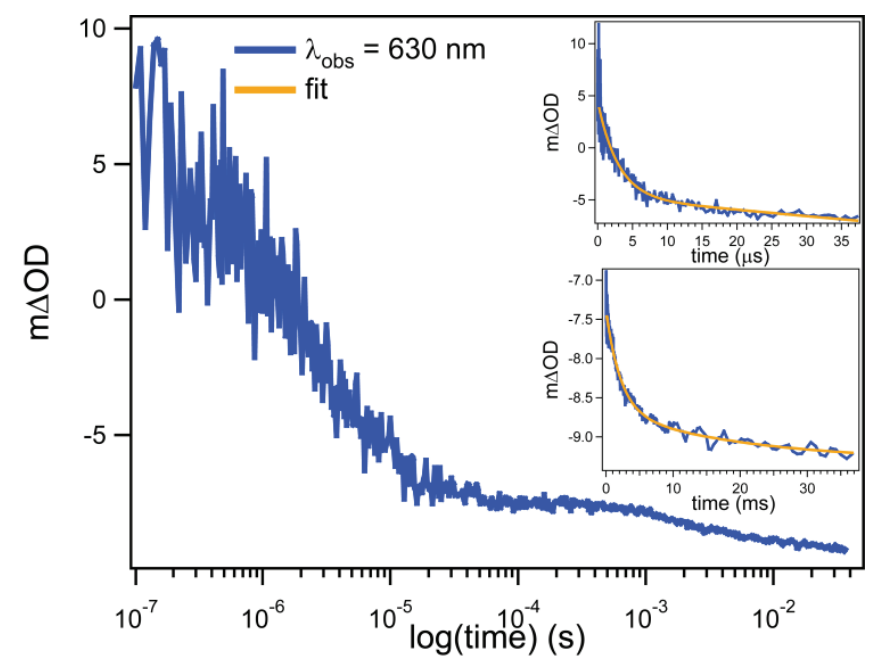

Figure 3.10 Typical transient kinetics trace for a sample containing $\quad 600 \quad \mu \mathrm{M} \quad$ 6-bromo-2-naphthol, $50 \mu \mathrm{M}$ $[\mathrm{Na}]\left[\mathrm{Co}^{\mathrm{I}}\left(\mathrm{dmgBF}_{2}\right)_{2}\left(\mathrm{CH}_{3} \mathrm{CN}\right)\right]$ and $100 \mathrm{mM} \quad\left[\mathrm{NBu}_{4}\right]\left[\mathrm{PF}_{6}\right]$ in logarithmic time $\left(\lambda_{\text {obs }}=630 \mathrm{~nm}\right)$. Two sequential kinetics processes were observed. Insets: Kinetics traces and fits plotted on a linear time axis. The faster process (top) fits a single exponential plus linear decay kinetics, the slower reaction (bottom) fits biexponential kinetics.

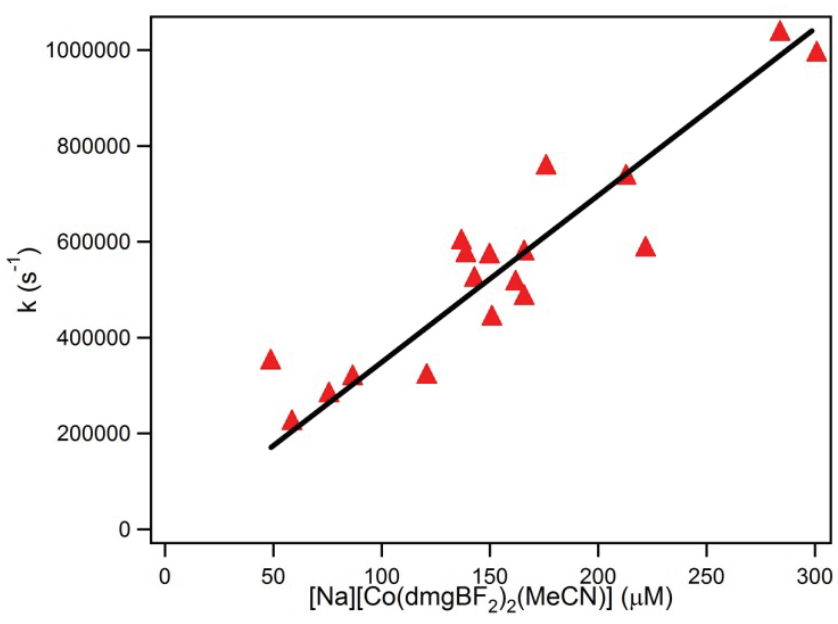

Figure 3.11 First-order rate constants for bleaching $\left[\mathrm{Co}\left(\mathrm{dmgBF}_{2}\right)_{2}\left(\mathrm{CH}_{3} \mathrm{CN}\right)\right]^{-}$absorption upon excitation of 6-bromo2-naphthol $\left(\lambda_{\mathrm{ex}}=266 \mathrm{~nm}, \lambda_{\mathrm{obs}}=630 \mathrm{~nm}\right)$. We found linear relationships between first-order rate constants and cobaloxime concentrations, allowing determination of a second-order rate constant for protonation. $k_{\mathrm{obs}}=k_{\mathrm{p}}\left[\mathrm{Co}\left(\mathrm{dmgBF}_{2}\right)_{2}\left(\mathrm{CH}_{3} \mathrm{CN}\right)\right]^{-}$, gives $k_{\mathrm{p}}=3.5 \times 10^{9} \mathrm{M}^{-1} \mathrm{~s}^{-1}$. 
The rate constant for protonation of $\left[\mathbf{C o}^{\mathrm{I}}\right]$ by ${ }^{3 *}\left[{ }^{3} \mathrm{NaphOH}\right]$ is a nearly diffusion-limited bimolecular process. Using digital simulations of cyclic voltammograms, Peters ${ }^{15}$ and Fontecave ${ }^{16}$ independently estimated the rate constant for protonation of electrochemically generated [ $\left.\mathrm{Co}^{\mathrm{I}}\right]$ by $p$-toluenesulfonic acid $\left(\mathrm{p} K_{\mathrm{a}}=8.0\right.$ in $\left.\mathrm{CH}_{3} \mathrm{CN}\right)$ and $p$-cyanoanilinium acid $\left(\mathrm{p} K_{\mathrm{a}}=7.6\right.$ in $\left.\mathrm{CH}_{3} \mathrm{CN}\right)$ to be between $7 \times 10^{3}$ and $1.5 \times 10^{5} \mathrm{M}^{-1} \mathrm{~s}^{-1}$. In related work, Bakac obtained a rate constant of $1.4 \times 10^{6} \mathrm{M}^{-1} \mathrm{~s}^{-1}$ for reaction with citrate in neutral citrate buffered aqueous solution. ${ }^{17}$ Based on the $\mathrm{p} K_{\mathrm{a}}$ of 14.6 estimated for ${ }^{3^{*}}\left[{ }^{\mathrm{r}} \mathrm{NaphOH}\right]$, the measured rate constant for protonation is anomalously large compared to the

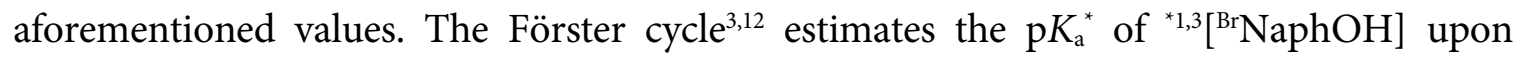
deprotonation to ${ }^{* 1,3}\left[{ }^{\mathrm{Br}} \mathrm{NaphOH}\right]$. However, an increase in apparent acidity beyond the Förster value to a $\mathrm{p} K_{\mathrm{a}}$ of -26.3 is expected if proton transfer from ${ }^{3 *}\left[{ }^{\mathrm{Br}} \mathrm{NaphOH}\right]$ to $\left[\mathrm{Co}^{\mathrm{I}}\right]$ is coupled to deactivation of the excited state (Figure 3.12), which accounts for the very high observed rate constant.

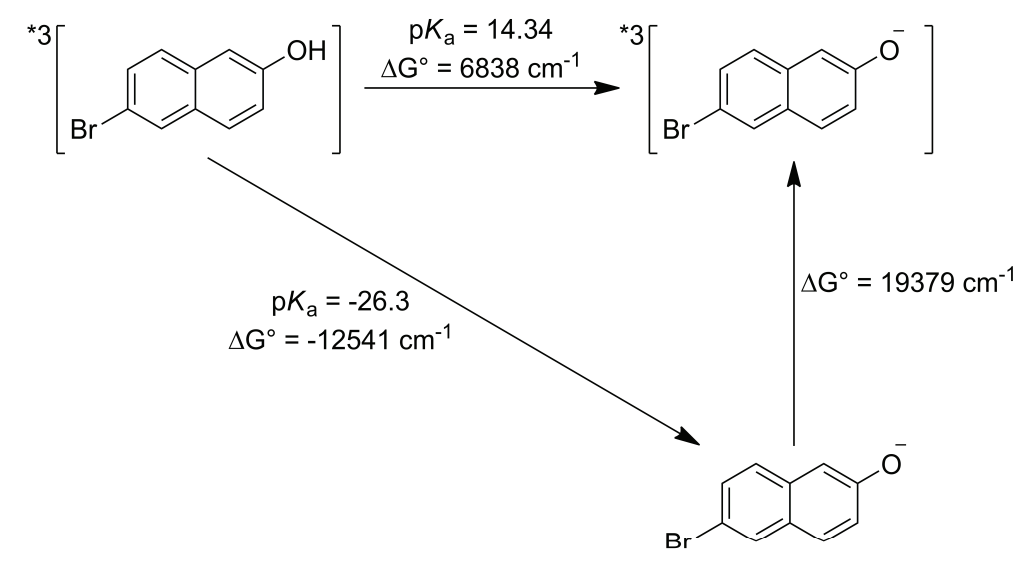

Figure 3.12 Energy cycle based on the Förster cycle predicted excited-state $\mathrm{p} K_{\mathrm{a}}$ and $\mathrm{E}_{0,0}\left(\mathrm{~T}_{1} \leftarrow \mathrm{S}_{0}\right)$ of 6-bromo-2-naphtholate. $\mathrm{p} K_{\mathrm{a}}$ values were converted to free energy via $\Delta G^{\circ}=-k_{B} \operatorname{Tn}\left(K_{a}\right)$.

To further probe the excited-state $\mathrm{p} K_{\mathrm{a}}$ of ${ }^{\mathrm{Br}} \mathrm{NaphOH}$, quenching by the conjugate base of $p$ toluenesulfonic acid $\left(\mathrm{p} K_{\mathrm{a}}=8.0\right.$ in $\left.\mathrm{CH}_{3} \mathrm{CN}\right)$, tetrabutylammonium tosylate $\left(\left[\mathrm{NBu}_{4}\right][\mathrm{OTs}]\right)$, 
was monitored by transient absorption measurements of ${ }^{3 *}\left[{ }^{\mathrm{Br}} \mathrm{NaphOH}\right]$ (Figure 3.13). As noted previously, the decay of ${ }^{*_{3}}\left[{ }^{\mathrm{Br}} \mathrm{NaphOH}\right]$ is biexponential, with rate constants of $6.4 \mathrm{x}$ $10^{4} \mathrm{~s}^{-1}$ and $4.0 \times 10^{5} \mathrm{~s}^{-1} .9$ In the presence of $\left[\mathrm{NBu}_{4}\right][\mathrm{OTs}],{ }^{3 *}\left[{ }^{\mathrm{Br}} \mathrm{NaphOH}\right]$ is quenched, consistent with a much lower $\mathrm{p} K_{\mathrm{a}}$ value than predicted by the Förster cycle (Figure 3.13, Figure 3.14). Based on the larger rate constant, a second-order rate constant for proton transfer is approximately $5.1 \times 10^{6} \mathrm{M}^{-1} \mathrm{~s}^{-1}$.

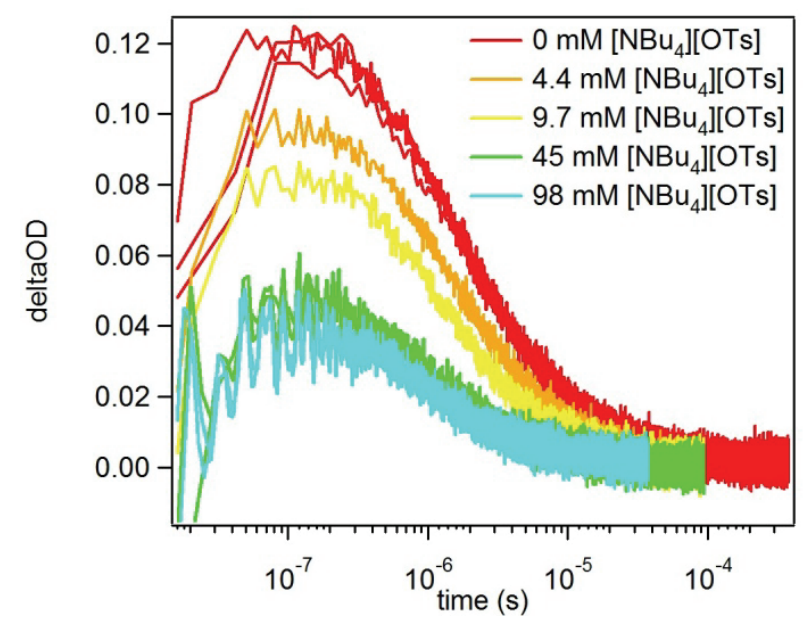

Figure 3.13 Kinetics traces monitoring the absorption of $3^{3^{*}}[6$ bromo-2-naphthol] at $460 \mathrm{~nm}\left(\lambda_{\mathrm{ex}}=266 \mathrm{~nm}\right)$ with varying concentrations of $\left[\mathrm{NBu}_{4}\right][\mathrm{OTs}]$. Kinetics traces are best fit to biexponential decay kinetics. 


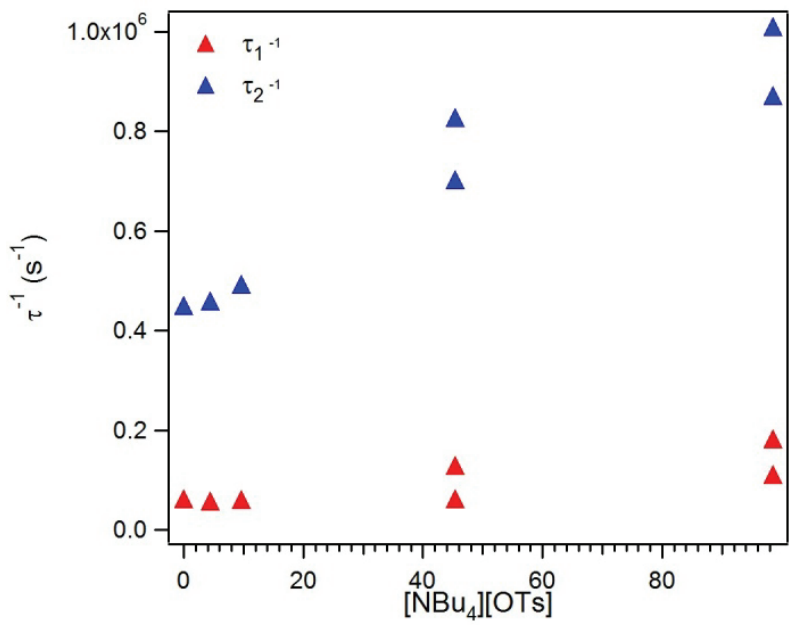

Figure 3.14 Rate constants for the biexponential decay of ${ }^{3^{*}}[6-$ bromo-2-naphthol] in the presence of [ $\left.\mathrm{NBu}_{4}\right][\mathrm{OTs}]$.

In the electrochemical analysis by Peters and coworkers, ${ }^{15}$ protonation was found to be rate limiting for catalysis. In our work, however, the rapid rate of protonation allows the kinetics of subsequent steps to be monitored. The slower process observed in transient measurements at $630 \mathrm{~nm}$ is best fit to a biexponential decay (Figure 3.10). When the concentration of $\left[\mathbf{C o}^{\mathrm{I}}\right]$ is varied, the rate constant for the faster component varies linearly while the second rate constant $\left(k \approx 100 \mathrm{~s}^{-1}\right)$ is independent of concentration. The plot of the observed first-order rate constant for the faster process versus $\left[\mathrm{Co}^{\mathrm{I}}\right]$ concentration gives a second-order rate constant of $9.2 \times 10^{6} \mathrm{M}^{-1} \mathrm{~s}^{-1}$ (Figure 3.15). 


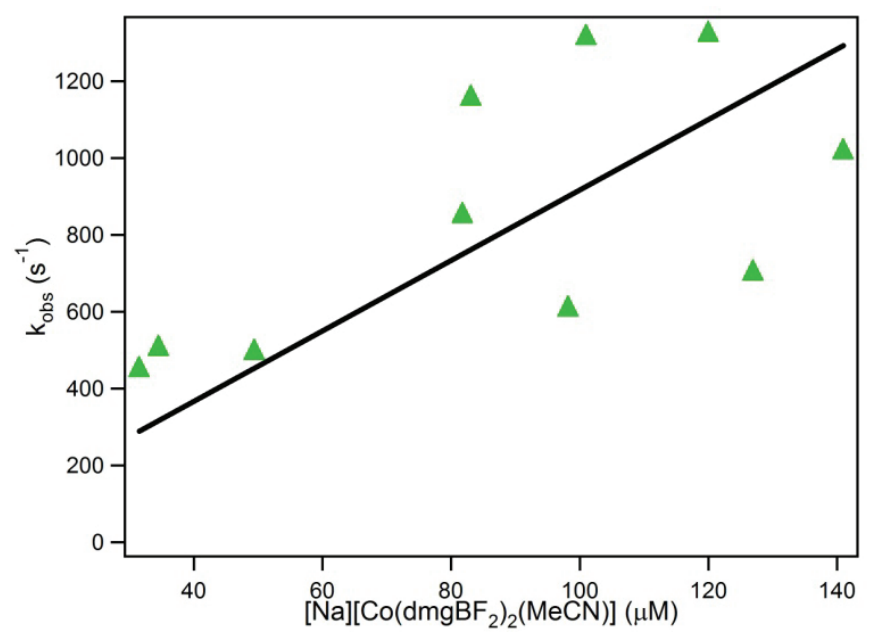

Figure 3.15 The slow kinetics process of kinetics traces measured at $630 \mathrm{~nm}$ was fit to a biexponential decay. The larger rate constant exhibits a linear relationship with the concentration of $\left[\mathrm{Co}\left(\mathrm{dmgBF}_{2}\right)_{2}\left(\mathrm{CH}_{3} \mathrm{CN}\right)\right]^{-}$. All samples contained $600 \mu \mathrm{M}$ 6bromo-2-Naphthol, $100 \mathrm{mM} \quad\left[\mathrm{NBu}_{4}\right]\left[\mathrm{PF}_{6}\right]$ and a varying concentration of $[\mathrm{Na}]\left[\mathrm{Co}\left(\mathrm{dmgBF}_{2}\right)_{2}\left(\mathrm{CH}_{3} \mathrm{CN}\right)\right]$. A linear fit to the data series, $k=k_{\text {red }}\left[\mathrm{Co}\left(\mathrm{dmgBF}_{2}\right)_{2}\left(\mathrm{CH}_{3} \mathrm{CN}\right)\right]^{-}$, gives $k_{\text {red }}=9.2 \times 10^{6}$ $\mathrm{M}^{-1} \mathrm{~s}^{-1}$.

Concurrent with the bleach of the $\left[\mathbf{C o}^{\mathrm{I}}\right]$ signal, a new absorption feature was observed in transient measurements between 380-450 nm. Attempts to obtain kinetics of formation of this intermediate were not successful, owing to the intense absorption of ${ }^{3 *}\left[{ }^{B r} \mathrm{NaphOH}\right]$ in this spectral region. On longer timescales, a transient signal monitored at $405 \mathrm{~nm}$ (Figure 3.16) decays to a more weakly absorbing final product, $\left[\mathbf{C o}{ }^{\mathrm{II}}\right]$. The conversion is best fit to single exponential decay with a rate constant of $\sim 100 \mathrm{~s}^{-1}$, matching the slowest kinetics process from $630 \mathrm{~nm}$ traces. Additional experiments revealed that the first-order rate constant for decay of this intermediate (monitored at $405 \mathrm{~nm}$ ) scales linearly with the concentration of ${ }^{\mathrm{Br}} \mathrm{NaphOH}$, giving a second-order rate constant of $4.2 \times 10^{4} \mathrm{M}^{-1} \mathrm{~s}^{-1}$. 


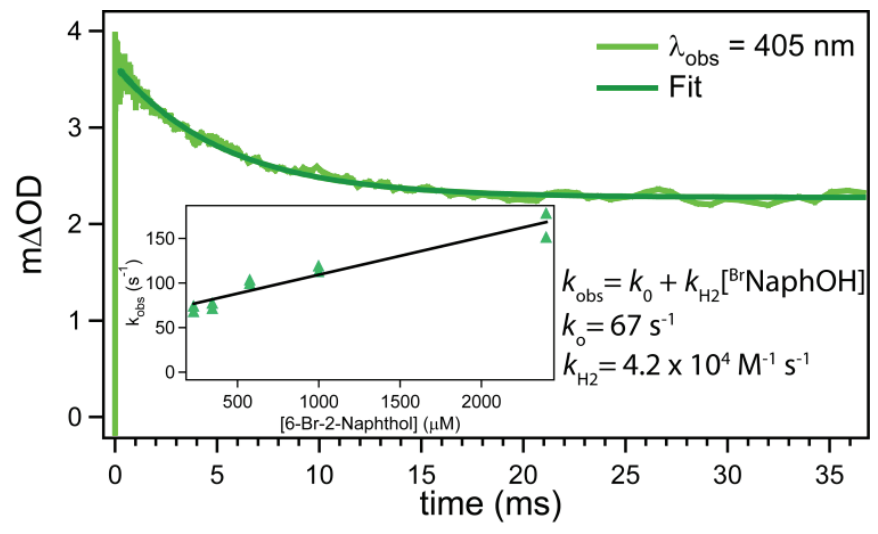

Figure 3.16 Typical transient kinetics trace at $405 \mathrm{~nm}$ for a sample containing $583 \mu \mathrm{M} \quad$ 6-bromo-2-naphthol, $263 \mu \mathrm{M}$ $[\mathrm{Na}]\left[\mathrm{Co}^{\mathrm{I}}\left(\mathrm{dmgBF}_{2}\right)_{2}\left(\mathrm{CH}_{3} \mathrm{CN}\right)\right]$ and $100 \mathrm{mM}\left[\mathrm{NBu}_{4}\right]\left[\mathrm{PF}_{6}\right]$ fits single exponential decay. Inset: Linear relationship between first-order rate constants for decay at $405 \mathrm{~nm}$ and the concentration of 6bromo-2-naphthol allows estimation of a second-order rate constant for $\mathrm{H}_{2}$ release. Conditions: $\sim 80 \mu \mathrm{M}$ $[\mathrm{Na}]\left[\mathrm{Co}^{\mathrm{I}}\left(\mathrm{dmgBF}_{2}\right)_{2}\left(\mathrm{CH}_{3} \mathrm{CN}\right)\right]$ and $100 \mathrm{mM}\left[\mathrm{NBu}_{4}\right]\left[\mathrm{PF}_{6}\right]$.

The transient absorption measurements are consistent with the kinetics model outlined in Scheme 3.2. Electronic excitation of ${ }^{\mathrm{Br}} \mathrm{NaphOH}$ promotes excited-state proton transfer to $\left[\mathbf{C o}^{\mathrm{I}}\right]$, forming $\left[\mathbf{C} \mathbf{o}^{\mathrm{III}} \mathbf{H}\right]$. The second kinetics phase correlates with reduction of transiently generated $\left[\mathbf{C o}^{\mathrm{III}} \mathbf{H}\right]$ by excess $\left[\mathbf{C o}^{\mathrm{I}}\right]$ to form a reduced hydride species, $\left[\mathrm{HCo}^{\mathrm{II}}\left(\mathrm{dmgBF}_{2}\right)_{2}\left(\mathrm{CH}_{3} \mathrm{CN}\right)\right]^{-}\left(\left[\mathbf{C o}^{\mathrm{II}} \mathbf{H}\right]\right)$ and $\left[\mathbf{C o}^{\mathrm{II}}\right]$. The $\left[\mathbf{C o}^{\mathrm{II}} \mathbf{H}\right]$ transient is a strongly absorbing yellow species with a weak spectral feature at $630 \mathrm{~nm}$. Protonation of this intermediate by weakly acidic ${ }^{\mathrm{Br}} \mathrm{NaphOH}$ releases $\mathrm{H}_{2}$ and generates [ $\left.\mathbf{C o}^{\mathrm{II}}\right]\left(k_{\mathrm{H} 2}=4.2 \times 10^{4}\right.$ $\left.\mathrm{M}^{-1} \mathrm{~s}^{-1}\right)$, indicating that $\left[\mathbf{C o}^{\mathrm{II}} \mathbf{H}\right]$ is strongly hydridic. A similar heterolytic route proceeding via $\left[\mathbf{C o}{ }^{\mathrm{II}} \mathbf{H}\right]$ likely is the dominate pathway in photocatalytic $\mathrm{H}_{2}$ evolution reactions reported by Eisenberg ${ }^{18-21}$ and Fontecave, ${ }^{22}$ where experimental conditions included both weakly basic solutions and high concentrations of powerful reductants. 


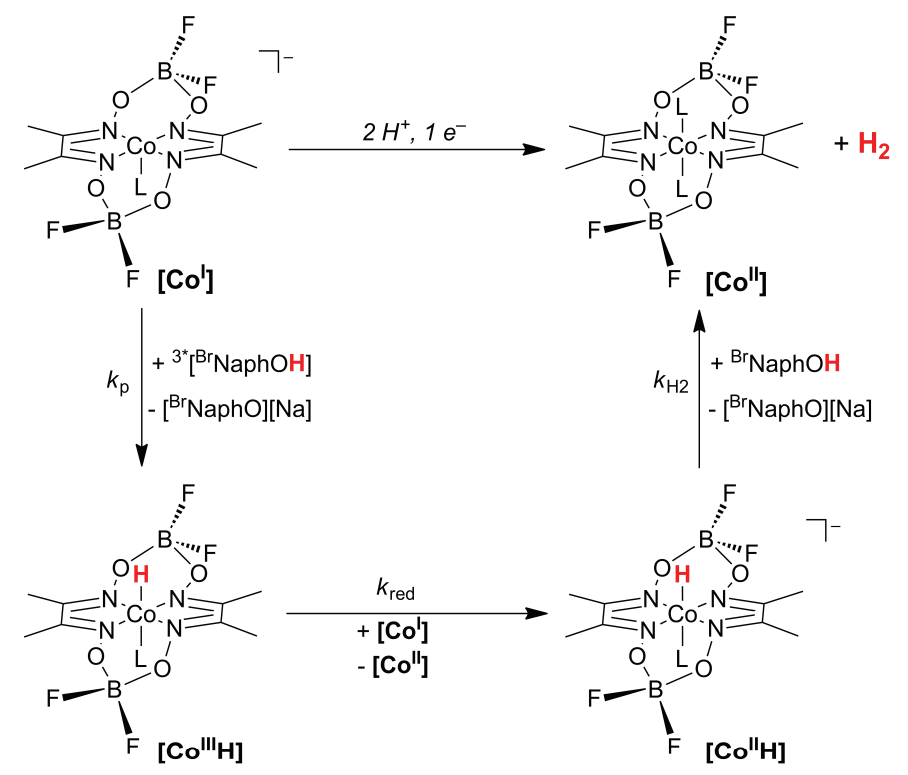

Scheme 3.2 Proposed kinetics model for $\mathrm{H}_{2}$ evolution.

The rate constant for reduction of $\left[\mathbf{C o}^{\mathrm{III}} \mathbf{H}\right]$ by $\left[\mathbf{C o}^{\mathrm{I}}\right]\left(k_{\text {red }}=9.2 \times 10^{6} \mathrm{M}^{-1} \mathrm{~s}^{-1}\right)$ corresponds to a barrier of 5-7 kcal mol-1. The work of Peters and coworkers is relevant here, as a quasireversible reduction peak in their cyclic voltammograms attributable to $\left[\mathbf{C o}^{\mathrm{III}} \mathbf{H}\right] /\left[\mathbf{C o}{ }^{\mathrm{II}} \mathbf{H}\right]$ reduction was observed $\sim 0.45 \mathrm{~V}$ negative of the $\left[\mathbf{C o}^{\mathrm{II}}\right] /\left[\mathbf{C o}^{\mathrm{I}}\right]$ reduction potential, implying a lower limit of $\sim 10.5 \mathrm{kcal} \mathrm{mol}^{-1}$ for the barrier to this reduction. ${ }^{15}$ Our work suggests that $\left[\mathrm{Co}^{\mathrm{III}} \mathbf{H}\right]$ is more readily reduced than previously thought.

Chao and Espenson obtained a second-order rate constant of $1.7 \times 10^{4} \mathrm{M}^{-1} \mathrm{~s}^{-1}$ for $\mathrm{H}_{2}$ production from an isolable phosphine stabilized hydride species, $\mathrm{HCo}(\mathrm{dmgH})_{2} \mathrm{P}\left(n-\mathrm{C}_{4} \mathrm{H}_{9}\right)_{3}$ $\left(\left[\mathrm{PCo}{ }^{\mathrm{III}} \mathrm{H}\right]\right),{ }^{23}$ a value that corresponds to a barrier of approximately $8-10 \mathrm{kcal} \mathrm{mol}^{-1}$ for bimolecular reductive elimination. As the hydride of the cobaloxime explored here $\left(\left[\mathbf{C o}{ }^{\mathrm{III}} \mathbf{H}\right]\right)$ is more reactive, ${ }^{15}$ a reasonable estimate for the analogous homolytic barrier would be 5-8 kcal mol${ }^{-1}$, similar to that observed for reduction of [ $\left.\mathbf{C o} \mathbf{~}^{\mathrm{III}} \mathbf{H}\right]$. However, under our experimental conditions, the concentration of $\left[\mathbf{C o}^{\mathrm{I}}\right]$ is significantly greater than that of 
the transiently generated $\left[\mathbf{C o}^{\mathrm{III}} \mathbf{H}\right]$. Thus a pathway in which the hydride is further reduced is favored.

\section{Conclusions}

We have demonstrated that photoacid triplet exited states can rapidly trigger protonation of metal complexes. Our work suggests that protonation of $\left[\mathbf{C o}^{\mathrm{I}}\right]$ by ${ }^{3^{*}}\left[{ }^{\mathrm{Br}} \mathrm{NaphOH}\right]$ is coupled to excited-state deactivation. The rapid rate of protonation has allowed us to study subsequent processes. Importantly, we found that transiently generated $\left[\mathrm{Co}^{\mathrm{III}} \mathbf{H}\right]$ is reduced by $\left[\mathbf{C o}^{\mathrm{I}}\right]$ to form $\left[\mathbf{C o}^{\mathrm{II}} \mathbf{H}\right]$, which is then protonated by ${ }^{\mathrm{Br}} \mathrm{NaphOH}$ to generate one equivalent of $\mathrm{H}_{2}$ and [Co' $\mathbf{C o}^{\mathrm{II}}$. Our experiments have revealed a hydrogen evolution pathway via protonation of $\left[\mathbf{C o}^{\mathrm{II}} \mathbf{H}\right]$ that is expected to dominate when low concentrations of $\left[\mathbf{C o}^{\mathrm{III}} \mathbf{H}\right]$ and an excess of reducing equivalents are present.

\section{Acknowledgement}

Bruce Brunschwig, Alec Durrell, Maraia Ener, Jonas Peters, and Jeff Warren provided helpful discussions and experimental advice. Charles McCrory and Jacob Good provided generous assistance with GC measurements. This work was supported by an NSF Center for Chemical Innovation (Powering the Planet, CHE-0947829), the Arnold and Mabel Beckman Foundation, and CCSER (Gordon and Betty Moore Foundation).

\section{Experimental Details}

\section{Reagents}

Syntheses of air and moisture sensitive compounds were carried out in a nitrogen atmosphere glovebox. Solvents for these syntheses were dried by a standard method ${ }^{24}$ or 
over activated sieves followed by passage over activated alumina. $\mathrm{CD}_{3} \mathrm{CN}$ was obtained from Cambridge Isotope Laboratories, Inc. All materials, unless noted, were used as received. Tetrabutylammonium hexafluorophosphate and 6-bromo-2-naphthol were purchased from Aldrich and recrystallized from ethanol. $\mathrm{Co}\left(\mathrm{dmgBF}_{2}\right)\left(\mathrm{CH}_{3} \mathrm{CN}\right)_{2}$ was prepared according to the method of Bakac and Espenson. ${ }^{25} \mathrm{MeCo}\left(\mathrm{dmgBF}_{2}\right)_{2}\left(\mathrm{CH}_{3} \mathrm{CN}\right)$ was prepared by the method of Ram et al. ${ }^{14}$

Samples for transient absorption and room temperature luminescence measurements were prepared in dry, degassed $\mathrm{CH}_{3} \mathrm{CN}$ and placed into the cell of a high-vacuum $1 \mathrm{~cm}$ pathlength fused quartz cuvette (Starna Cells) connected to a $10 \mathrm{~mL}$ bulb and isolated from atmosphere and the bulb by a high-vacuum Teflon valve (Kontes). Samples for low temperature luminescence measurements were prepared in dry, degassed butyronitrile and placed in $1 \mathrm{~cm}$ diameter glass tubes sealed with a high-vacuum Teflon valve.

$[\mathrm{Na}]\left[\mathrm{Co}\left(\mathrm{dmgBF}_{2}\right)_{2}\left(\mathrm{CH}_{3} \mathrm{CN}\right)\right]$ was prepared in an analogous method to the literature preparation for $[\mathrm{Na}]\left[\mathrm{Co}\left(\mathrm{dpgBF}_{2}\right)_{2}\left(\mathrm{CH}_{3} \mathrm{CN}\right)\right] \cdot{ }^{15} \quad 0.251 \mathrm{~g} \quad(0.559 \mathrm{mmol}, 1$ eq. $)$ of $\mathrm{Co}\left(\mathrm{dmgBF}_{2}\right)\left(\mathrm{CH}_{3} \mathrm{CN}\right)_{2}$ was suspended in $4 \mathrm{~mL}$ of $\mathrm{THF}$ and transferred to a $20 \mathrm{~mL}$ scintillation vial containing $2.673 \mathrm{~g}$ (0.579 mmol, 1.04 eq.) of sodium mercury amalgam $(0.5 \% \mathrm{Na})$. The reaction was stirred for 3 hours to yield a deep blue-black solution. Solution was removed from the mercury and remaining blue solid was dissolved in $\mathrm{CH}_{3} \mathrm{CN}$ and removed. THF and $\mathrm{CH}_{3} \mathrm{CN}$ solutions were mixed and the solvent was reduced to $<1$ mL. $15 \mathrm{~mL}$ of $\mathrm{Et}_{2} \mathrm{O}$ was added to precipitate a blue solid, which was collected via filtration on medium frit. Product was dried under vacuum. Yield $=71 \%$. ${ }^{1} \mathrm{HNMR}\left(\mathrm{CD}_{3} \mathrm{CN}, 300\right.$ $\mathrm{MHz}): \delta 2.09(\mathrm{~s}, 12 \mathrm{H}), \delta 1.96(\mathrm{~s}, 3 \mathrm{H})$.

Sodium 6-bromo-2-naphtholate In an inert atmosphere, a $20 \mathrm{~mL}$ scintillation vial was charged with $21 \mathrm{mg}(0.883 \mathrm{mmol})$ sodium hydride and a stirbar. A solution of $197 \mathrm{mg}$ 
(0.883 mmol) of 6-bromo-2-naphthol in $4 \mathrm{~mL}$ dry, degassed acetonitrile was transferred to the reaction vessel. A white suspension was immediately formed and vigorous bubbling was observed. Reaction was allowed to stir for 1 hour, until solution became clear pale yellow. Solution was filtered and solvent was removed. Yield $=90 \%$. ${ }^{1} \mathrm{H}$ NMR $\left(\mathrm{CD}_{3} \mathrm{CN}, 300\right.$ $\mathrm{MHz}): \delta 7.67(\mathrm{~d}, \mathrm{~J}=1.2 \mathrm{~Hz}, 1 \mathrm{H}), \delta 7.39(\mathrm{~d}, \mathrm{~J}=8.7 \mathrm{~Hz}, 1 \mathrm{H}), \delta 7.18(\mathrm{~m}, 2 \mathrm{H}), \delta 6.92\left(\mathrm{dd}, \mathrm{J}_{1}=\right.$ $\left.8.9 \mathrm{~Hz}, \mathrm{~J}_{2}=2.6 \mathrm{H}, 1 \mathrm{H}\right), \delta 6.66(\mathrm{~d}, \mathrm{~J}=2.4 \mathrm{~Hz}, 1 \mathrm{H})$

\section{Bulk Photolysis}

Bulk photolysis experiments were utilized to characterize the evolution of hydrogen from excitation of 6-bromo-2-naphthol in the presence of $[\mathrm{Na}]\left[\mathrm{Co}\left(\mathrm{dmgBF}_{2}\right)_{2}\left(\mathrm{CH}_{3} \mathrm{CN}\right)\right]$. Reactions were run in a custom built $61 \mathrm{~mL}$ round-bottom flask fitted with a 1 inch diameter quartz window, 24/40 ground glass joint, Kontes valve leading to a 14/20 joint sealed with a septum. In a glovebox, the reaction vessel was charged with $5 \mathrm{mM}$ 6-bromo-2naphthol, $50 \mathrm{mM} \mathrm{NBu} 4 \mathrm{PF}_{6}$, and the noted amount of $[\mathrm{Na}]\left[\mathrm{Co}\left(\mathrm{dmgBF}_{2}\right)_{2}\left(\mathrm{CH}_{3} \mathrm{CN}\right)\right]$ in 40 $\mathrm{mL}$ acetonitrile with a stirbar and the vessel was sealed with a 24/40 glass stopper and Kontes valve. The sample was placed in front of a $100 \mathrm{~mW} 266 \mathrm{~nm} \mathrm{Nd:YAG} \mathrm{laser} \mathrm{and}$ photolyzed while stirring for approximately 45 minutes until the blue $[\mathrm{Na}]\left[\mathrm{Co}\left(\mathrm{dmgBF}_{2}\right)_{2}\left(\mathrm{CH}_{3} \mathrm{CN}\right)\right]$ had reacted to form yellow $\mathrm{Co}\left(\mathrm{dmgBF}_{2}\right)_{2}\left(\mathrm{CH}_{3} \mathrm{CN}\right)_{2}$.

The amount of $\mathrm{H}_{2}$ produced evolved was quantified by analyzing the gas mixture in the headspace using an Agilent 7890A GC-TCD. The total amount of $\mathrm{H}_{2}$ produced was calculated as a sum of the $\mathrm{H}_{2}$ in the headspace plus the $\mathrm{H}_{2}$ dissolved in the solvent (calculated using Henry's Law with a constant of $290 \mathrm{~L} \bullet \mathrm{atm} / \mathrm{mol}$ ). 
Physical Methods

NMR spectra were recorded using a Varian Mercury 300 spectrometer. ${ }^{1} \mathrm{H}$ NMR chemical shifts were referenced to residual solvents as determined relative to $\mathrm{Me}_{4} \mathrm{Si}(\delta=0 \mathrm{ppm})$. UVvisible absorption measurements were carried out using a Hewlett Packard 8452 or Cary 50 $\mathrm{UV}$-Vis spectrophotometer in $1 \mathrm{~cm}$ pathlength quartz cuvettes.

Steady-state and time-resolved spectroscopic measurements were carried out at the Beckman Institute Laser Resource Center (California Institute of Technology). Emission spectra were recorded on a Jobin Yvon Spec Fluorolog-3-11. Sample excitation was achieved via a xenon arc lamp with wavelength selection provided by a monochromator. Right angle emission was sorted using a monochromator and detected with a Hamamatsu R928P photomultiplier tube with photon counting. Appropriate long pass filters were utilized to minimize scattered excitation wavelength. Low temperature measurements were made in a liquid nitrogen filled glass finger dewar aligned in the fluorometer sample cavity.

For time-resolved measurements, $266 \mathrm{~nm}$ laser excitation was provided by $8 \mathrm{~ns}$ pulses from the fourth harmonic of a $10 \mathrm{~Hz}$ Q-switched Nd:YAG laser (Spectra-Physics Quanta-Ray PRO-Series). Probe light for transient absorption kinetics measurements was provided by a $75 \mathrm{~W}$ arc lamp (PTI Model A 1010) that could be operated in continuous wave or pulsed modes. Timing between the laser and the probe light was controlled by a digital delay generator. After passing through the sample collinearly with the laser beam, scattered excitation light was rejected by suitable long pass and short pass filters, and probe wavelengths were selected for detection by a double monochromator (Instruments SA DH10) with $1 \mathrm{~mm}$ slits. Transmitted light was detected with a photomultiplier tube (PMT, Hamamatsu R928). The PMT current was amplified and recorded with a GageScope transient digitizer. The data were converted to units of $\Delta \mathrm{OD}\left(\Delta \mathrm{OD}=-\log _{10}\left(\mathrm{I} / \mathrm{I}_{0}\right)\right.$, where $\mathrm{I}$ is 
the time-resolved probe-light intensity with laser excitation, and $I_{0}$ is the intensity without excitation). Samples measured on the microsecond timescale or faster were stirred continuously and measured using a laser repetition rate of $10 \mathrm{~Hz}$, while samples measured on a millisecond timescale were excited with a single shutter-released laser pulse, stirred for $1 \mathrm{~s}$ after collecting data, then allowed to sit until the solution settled $(2 \mathrm{~s})$ before the next laser pulse. Data were averaged over approximately 50 shots. All instruments and electronics in these systems were controlled by software written in LabVIEW (National Instruments). Data were log-time compressed prior to fitting. In certain cases, data from separate timescale measurements were stitched together (Appendix C). Data manipulation was performed with MATLAB R2008a (Mathworks, Inc.) and graphed with Igor Pro 5.01 (Wavemetrics).

\section{Calculations of Barriers}

Barriers were estimated as $\Delta G^{\ddagger}=-\mathrm{RT} \ln \left(\frac{k}{\mathrm{z}}\right)$

$k=$ rate constant, $\mathrm{M}^{-1} \mathrm{~s}^{-1}$

$\mathrm{Z}=$ collision factor, $10^{11} \mathrm{M}^{-1} \mathrm{~s}^{-1}$

$\mathrm{R}=$ molar gas constant, $0.001986 \mathrm{kcal} \mathrm{K}^{-1} \mathrm{~mol}^{-1}$

$\mathrm{T}=$ temperature, $298 \mathrm{~K}$

\section{References}

1. Dempsey, J. L.; Brunschwig, B. S.; Winkler, J. R.; Gray, H. B. Acc. Chem. Res. 2009, 42, 1995.

2. Dempsey, J. L.; Winkler, J. R.; Gray, H. B. J. Am. Chem. Soc. 2010, 132, 1060.

3. Forster, T. Z. Elektrochem. 1950, 54, 531. 
4. Tolbert, L. M.; Solntsev, K. M. Acc. Chem. Res. 2001, 35, 19.

5. Arnaut, L. G.; Formosinho, S. J. J. Photochem. Photobiol. A 1993, 75, 1.

6. Gutman, M.; Huppert, D. J. Biochem. Bioph. Methods 1979, 1, 9.

7. McClure, D. S. J. Chem. Phys. 1949, 17, 905.

8. McClure, D. S.; Blake, N. W.; Hanst, P. L. J. Chem. Phys. 1954, 22, 255.

9. Pretali, L.; Doria, F.; Verga, D.; Profumo, A.; Freccero, M. J. Org. Chem. 2009, 74, 1034.

10. Bordwell, F. G.; Cheng, J. J. Am. Chem. Soc. 1991, 113, 1736.

11. Kütt, A.; Leito, I.; Kaljurand, I.; Sooväli, L.; Vlasov, V. M.; Yagupolskii, L. M.; Koppel, I. A. J. Org. Chem. 2006, 71, 2829.

12. Grabowski, Z. R.; Rubaszewska, W. J. Chem. Soc., Faraday Trans. 2 1977, 73, 11.

13. Fourmond, V.; Jacques, P.-A.; Fontecave, M.; Artero, V. Inorg. Chem. 2010, null.

14. Ram, M. S.; Riordan, C. G.; Yap, G. P. A.; LiableSands, L.; Rheingold, A. L.; Marchaj, A.; Norton, J. R. J. Am. Chem. Soc. 1997, 119, 1648.

15. Hu, X.; Brunschwig, B. S.; Peters, J. C. J. Am. Chem. Soc. 2007, 129, 8988.

16. Baffert, C.; Artero, V.; Fontecave, M. Inorg. Chem. 2007, 46, 1817.

17. Szajna-Fuller, E.; Bakac, A. Eur. J. Inorg. Chem. 2010, 2010, 2488.

18. Du, P.; Knowles, K.; Eisenberg, R. J. Am. Chem. Soc. 2008, 130, 12576.

19. Du, P.; Schneider, J.; Luo, G.; Brennessel, W. W.; Eisenberg, R. Inorg. Chem. 2009, 48, 4952.

20. Lazarides, T.; McCormick, T.; Du, P.; Luo, G.; Lindley, B.; Eisenberg, R. J. Am. Chem. Soc. 2009, 131, 9192.

21. McCormick, T. M.; Calitree, B. D.; Orchard, A.; Kraut, N. D.; Bright, F. V.; Detty, M. R.; Eisenberg, R. J. Am. Chem. Soc. 2010, 132, 15480.

22. Fihri, A.; Artero, V.; Pereira, A.; Fontecave, M. Dalton Trans. 2008, 5567.

23. Chao, T.-H.; Espenson, J. H. J. Am. Chem. Soc. 1978, 100, 129. 
24. Pangborn, A. B.; Giardello, M. A.; Grubbs, R. H.; Rosen, R. K.; Timmers, F. J. Organometallics 1996, 15, 1518.

25. Bakac, A.; Espenson, J. H. J. Am. Chem. Soc. 1984, 106, 5197. 
Chapter 4

MECHANISTIC INVESTIGATIONS OF COBALOXIME CATALYZED $\mathrm{H}_{2}$ EVOLUTION IN AQUEOUS MEDIA 


\section{Introduction}

While reports of $\mathrm{H}_{2}$ evolution catalysts are not uncommon, only a handful of these reported complexes operate in pure aqueous media. These systems that catalyze $\mathrm{H}_{2}$ evolution in water include $\left.\left[\mathrm{PY}_{5} \mathrm{Me}_{2}\right) \mathrm{MoO}\right]^{2+}\left(\mathrm{PY}^{2} \mathrm{Me}_{2}=\right.$ 2,6-bis(1,1-bis(2-pyridyl)ethyl)pyridine $){ }^{1}$ $\mathrm{Ni}(\text { cyclam })^{2+},{ }^{2} \mathrm{Co}(\text { bpy })_{3}{ }^{2+}$ (bpy $=2,2$-bipyridine $),{ }^{3}$ as well as several water soluble $\mathrm{Co}^{\mathrm{II}}$ (porphyrin) complexes ${ }^{4}$ and other cobalt(II) complexes with nitrogen-based macrocycles, ${ }^{5-7}$ including a cobaloxime central to this thesis, $\mathrm{Co}(\mathrm{dmgBF})_{2}\left(\mathrm{H}_{2} \mathrm{O}\right)_{2} \quad(\mathbf{1}$, $\mathrm{dmgBF}_{2}=$ difluoroboryl-dimethylglyoxime, $\left.\left[\mathrm{Co}^{\mathrm{II}}\right]\right){ }^{8-9}$ Connolly and Espenson made the first report of cobaloxime catalyzed hydrogen evolution in aqueous media in $1986,{ }^{8}$ noting that $\left[\mathrm{Co}^{\mathrm{II}}\right]$ catalyzed the reduction of $\mathrm{HCl}_{\mathrm{aq}}$ utilizing $\mathrm{Cr}^{2+}{ }_{\text {aq }}$ as a stoichiometric reductant. Berben and Peters have since reported the electrocatalytic evolution of $\mathrm{H}_{2}$ by [Co ${ }^{\mathrm{II}}$ ] in phosphate-buffered solutions, both in homogeneous solutions and with the catalyst adsorbed on a glassy carbon electrode..$^{10}$ Samples of [C $\left.\mathbf{C} \mathbf{o}^{\mathrm{II}}\right]$ dissolved in phosphate-buffered solutions at $\mathrm{pH} 7$ exhibit an irreversible $\mathrm{Co}^{\mathrm{III} / \mathrm{II}}$ redox process at $0.09 \mathrm{~V}$ vs. SCE and a reversible $\mathrm{Co}^{\mathrm{II} / \mathrm{I}}$ redox couple at $-0.70 \mathrm{~V}$ vs. SCE. As the $\mathrm{pH}$ of solution is lowered, an increase in current is observed at $-0.95 \mathrm{~V}$ vs. SCE (Figure 4.1), indicative of a catalytic process. Unlike electrochemical measurements in non-aqueous media, ${ }^{9,11-13}$ the catalytic currents at potentials significantly negative of the $\mathrm{Co}^{\mathrm{II} / \mathrm{I}}$ potential, suggesting the mechanism in water is distinct from that in organic solvent. 


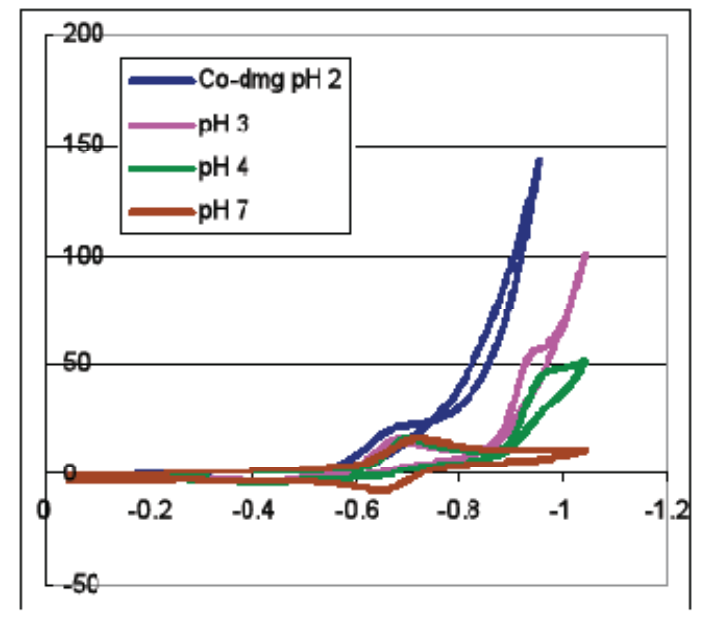

Figure 4.1 Cyclic voltammograms (current $(\mu \mathrm{A})$ vs. Potential $(\mathrm{V}$ vs. SCE)) recorded for $\mathrm{Co}\left(\mathrm{dmgBF}_{2}\right)_{2}\left(\mathrm{H}_{2} \mathrm{O}\right)_{2}$ in phosphate buffered solutions at various pHs. From Reference 10.

To further explore the mechanism of proton reduction by $\left[\mathbf{C o}^{\mathrm{II}}\right]$, photoionization and pulse radiolysis triggering methods have been utilized in conjunction with time-resolved spectroscopy to examine the mechanistic pathways responsible for $\mathrm{H}_{2}$ evolution in water. Both methods are based on the chemistry of the solvated electron, $e_{\mathrm{aq}}{ }^{-}$, a potent reductant with an estimated reduction potential at $-2.7 \mathrm{~V} .{ }^{14}$

\section{Results and Discussion}

\section{Photoionization of NADH}

Two-photon excitation $\left(\lambda_{\mathrm{ex}}=355 \mathrm{~nm}\right)$ of $\mathrm{NADH}$ (nicotinamide adenine dinucleotide, reduced form) leads to the ejection of a $e_{\mathrm{aq}}{ }^{-}$to yield $\mathrm{NADH}^{\bullet+}$, which releases a proton to the bulk solution to yield a second powerful reducing species, NAD• (-0.94 V) (Scheme 4.1). ${ }^{15-16}$ 


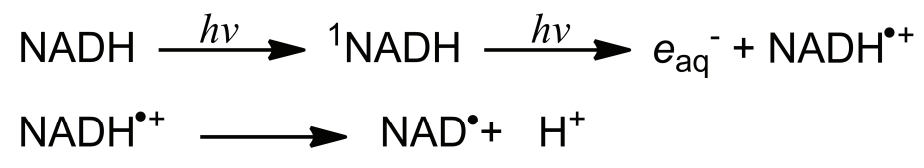

Scheme 4.1

This photoionization method was used previously to phototrigger redox processes in biological systems..$^{15,17-19}$ Here, photoionization of NADH triggered the reduction of [Co' $\left.{ }^{I I}\right]$ to $\left[\mathrm{Co}\left(\mathrm{dmgBF}_{2}\right)_{2}\left(\mathrm{H}_{2} \mathrm{O}\right)\right]^{-}\left(\left[\mathbf{C o}^{\mathrm{I}}\right]\right)$. In samples containing $\left[\mathbf{C o}^{\mathrm{II}}\right]$ and $\mathrm{NADH}$ in neutral water, a buildup of the reduced cobaloxime was observed in absorption spectra measured upon bulk photolysis with $355 \mathrm{~nm}$ excitation. The appearance of the [Co $\left.{ }^{\mathrm{I}}\right]$ signal was monitored at $630 \mathrm{~nm}$; transient kinetics traces were fit to single exponentials (Figure 4.2). A secondorder rate constant for reduction of $\left[\mathrm{Co}^{\mathrm{I}}\right]$ by the photoproduced reducing equivalents $\left(e_{\mathrm{aq}}^{-}\right.$ and NAD•) of roughly $7.4 \times 10^{7} \mathrm{M}^{-1} \mathrm{~s}^{-1}$ was estimated.

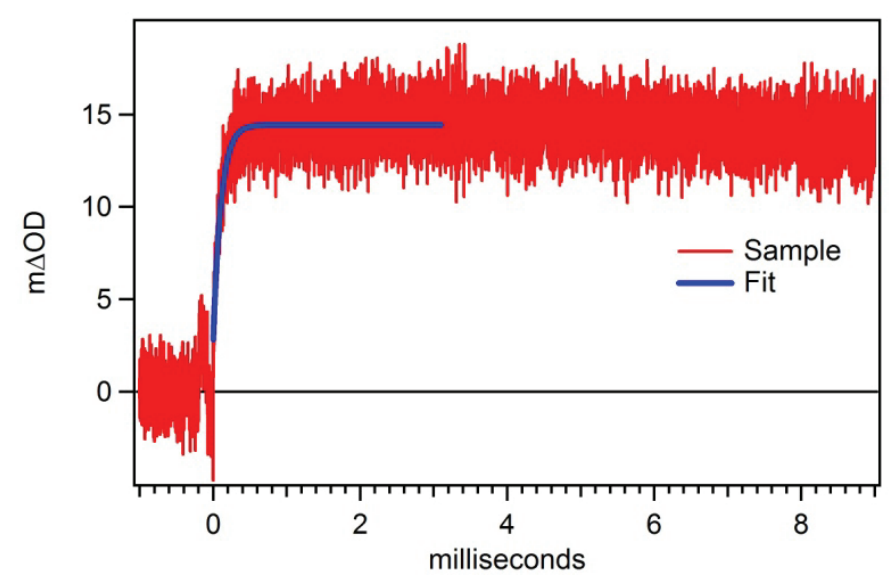

Figure 4.2 Kinetics trace observed at $630 \mathrm{~nm}$ for the reaction of $\mathrm{Co}\left(\mathrm{dmgBF}_{2}\right)_{2}\left(\mathrm{H}_{2} \mathrm{O}\right)_{2}+\mathrm{e}_{\mathrm{aq}}{ }^{-}$formed via photoionization of $\mathrm{NADH}$, fit to first-order exponential $\left(k_{\mathrm{obs}}=9.4 \times 10^{3} \mathrm{~s}^{-1}\right)$. A second-order rate constant for the reaction was determined to be $7.4 \times 10^{7} \mathrm{M}^{-1} \mathrm{~s}^{-}$ ${ }^{1}$. Conditions: $610 \mu \mathrm{M} N A D H, 128 \mu \mathrm{M} \mathrm{Co}\left(\mathrm{dmgBF}_{2}\right)_{2}\left(\mathrm{H}_{2} \mathrm{O}\right)_{2}$ in neutral water, $\lambda_{\mathrm{ex}}=355 \mathrm{~nm}$. 
Unfortunately, NADH undergoes a rapid and irreversible reaction in acidic solutions to form a hydrolyzed product that can be optically monitored. ${ }^{20-22}$ Ultimately, this acidcatalyzed degradation process obstructed the proposed studies of the reactivity of [Col] with protons and a more acid robust photoionization system was sought.

\section{Photoionization of Indole}

Photoionization of indole and tryptophan $\left(\lambda_{\mathrm{ex}}=220-290 \mathrm{~nm}\right)$ in aqueous solution generates $e_{\mathrm{aq}}{ }^{-}$primarily via a monophotonic process, which can either reduce $\left[\mathbf{C o}{ }^{\mathrm{II}}\right]$ directly or reduce indole or tryptophan to the corresponding anions, also potent reductants (Scheme 4.2). ${ }^{23-25}$

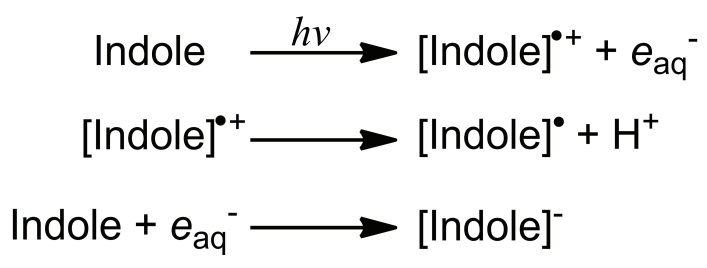

Scheme 4.2

Transient absorption spectroscopy was used to monitor the reactivity of $e_{\mathrm{aq}}{ }^{-}$, produced via

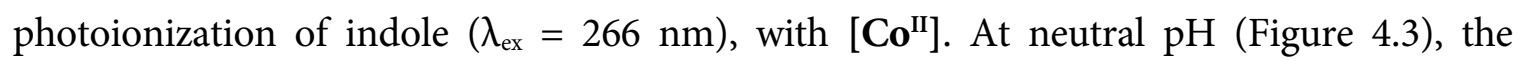
transient difference spectrum collected $25 \mu$ s after photoionization with a diode array spectrometer shows a new absorption centered at $606 \mathrm{~nm}$ consistent with formation of $\left[\mathbf{C o}^{\mathrm{I}}\right]$, as well as a bleach at $456 \mathrm{~nm}$, indicative of $\left[\mathbf{C o}^{\mathrm{II}}\right]$ consumption. Charge recombination reactions of $\left[\mathbf{C o}^{\mathrm{I}}\right]$ with the oxidized indole products occurs on the millisecond timescale. At pH 3 (Figure 4.4), the transient difference spectrum shows no absorption indicative of $\left[\mathbf{C o}^{\mathrm{I}}\right]$ formation at $25 \mu$ s delays or longer. A persistent bleach centered at $456 \mathrm{~nm}$ consistent with consumption of [C $\left.\mathbf{C o}^{\mathrm{II}}\right]$ is observed at time delays 
between $25 \mu$ s and $50 \mathrm{~ms}$, and steady-state absorption spectra observed at photolysis intervals indicate irreversible consumption of $\left[\mathbf{C o}^{\mathrm{II}}\right]$. Several explanations for this behavior were considered, and the irreversible reactivity of $\left[\mathrm{Co}^{\mathrm{II}}\right]$ is likely due to a combination of these processes. First, formation of the desired hydride species, $\mathrm{HCo}\left(\mathrm{dmgBF}_{2}\right)_{2}\left(\mathrm{H}_{2} \mathrm{O}\right)$ [Co $\left.{ }^{\mathrm{III}} \mathbf{H}\right]$ (which has no strong absorption in this region), could arise via two pathways; reduction of $\left[\mathbf{C o}^{\mathrm{II}}\right]$ followed by protonation or reactivity of $\left[\mathrm{Co}^{\mathrm{II}}\right]$ with $\mathrm{H} \bullet$, formed via proton scavenging of $e_{\mathrm{aq}}{ }^{-}$. Low concentrations of $\left[\mathbf{C} \mathbf{o}^{\mathrm{III}} \mathbf{H}\right]$ could impede a bimolecular pathway for hydrogen evolution or, as suggested by the electrochemistry, additional reduction may be necessary for $\mathrm{H}_{2}$ evolution. However, even if additional reduction of $\left[\mathrm{Co}^{\mathrm{III}} \mathbf{H}\right]$ to form $\left[\mathbf{C} \mathbf{o}^{\mathrm{II}} \mathbf{H}\right]$ is necessary, the abundance of reducing equivalents available upon bulk photoionization of indole should open up a catalytic pathway for $\mathrm{H}_{2}$ evolution, regenerating $\left[\mathbf{C o}^{\mathrm{II}}\right]$. Further, acidic conditions may catalyze decomposition pathways triggered by cobaloxime reduction and, lastly, scavenging of $e_{\mathrm{aq}}^{-}$by protons could limit reducing equivalents available for cobaloxime reduction. 


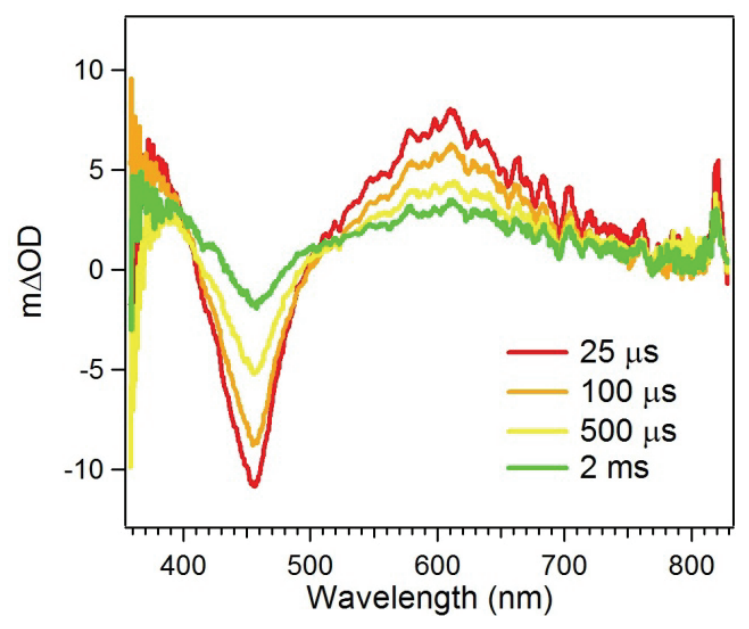

Figure 4.3 Transient difference spectra at $\mathrm{pH} 7$ for the reaction of $\mathrm{Co}\left(\mathrm{dmgBF}_{2}\right)_{2}\left(\mathrm{H}_{2} \mathrm{O}\right)_{2}$ with $e_{\mathrm{aq}}{ }^{-}$, formed via photoionization of indole. Conditions: $1 \mathrm{mM}$ indole, $10 \mathrm{mM} \mathrm{pH} 7 \mathrm{KPi}$ at $100 \mathrm{mM}$ ionic strength $\left(\mathrm{Na}_{2} \mathrm{SO}_{4}\right), 136 \mu \mathrm{M} \mathrm{Co}\left(\mathrm{dmgBF}_{2}\right)_{2}\left(\mathrm{H}_{2} \mathrm{O}\right)_{2}, \lambda_{\text {ex }}=266$ $\mathrm{nm}$.

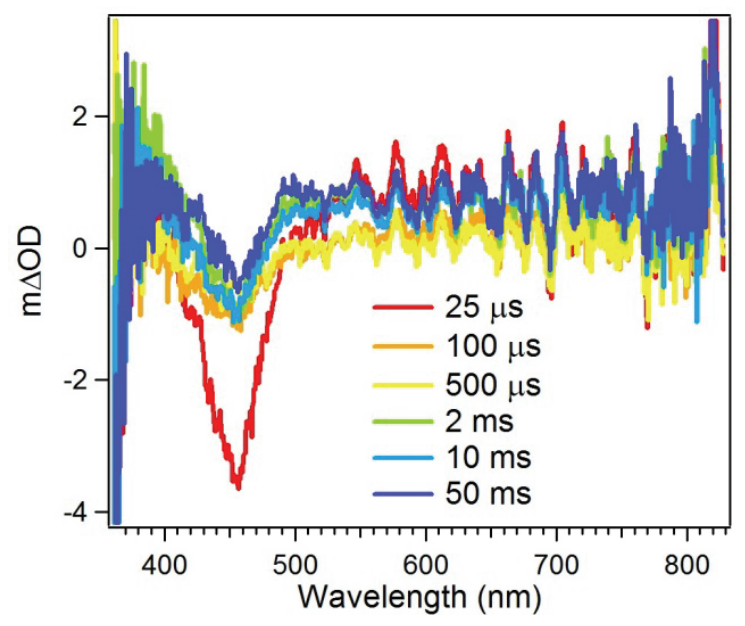

Figure 4.4 Transient difference spectra at $\mathrm{pH} 3$ for the reaction of $\mathrm{Co}\left(\mathrm{dmgBF}_{2}\right)_{2}\left(\mathrm{H}_{2} \mathrm{O}\right)_{2}$ with $e_{\mathrm{aq}}^{-}$, formed via photoionization of indole. Conditions: $1 \mathrm{mM}$ indole, $10 \mathrm{mM} \mathrm{pH} 3 \mathrm{KP} i$ at $100 \mathrm{mM}$ ionic strength $\left(\mathrm{Na}_{2} \mathrm{SO}_{4}\right), 144 \mu \mathrm{M} \mathrm{Co}\left(\mathrm{dmgBF}_{2}\right)_{2}\left(\mathrm{H}_{2} \mathrm{O}\right)_{2}, \lambda_{\text {ex }}=266$ $\mathrm{nm}$. 
Ultimately, a clear interpretation of the kinetics of cobaloxime reactivity with $e_{\mathrm{aq}}{ }^{-}$at low $\mathrm{pH}$ is complicated by multiple unproductive pathways that consume $e_{\mathrm{aq}}{ }^{-}$and the cobaloxime. Additional experiments are warranted, but data interpretation is likely to be challenging.

\section{Pulse Radiolysis}

In pulse radiolysis experiments, a Van de Graaff accelerator generates pulses of high-energy electrons which initiate the ionization of water to primarily yield solvated electrons $\left(e_{\mathrm{aq}}{ }^{-}\right)$ and $\bullet \mathrm{OH}$ (Scheme 4.3). In typical experiments, $\bullet \mathrm{OH}$ is scavenged and the $e_{\mathrm{aq}}{ }^{-}$provides a reducing equivalent, either directly or indirectly.

$$
\mathrm{H}_{2} \mathrm{O} \stackrel{2 \mathrm{MeV} \text { electrons }}{\longrightarrow} e_{\mathrm{aq}}^{-}, \cdot \mathrm{OH},\left(\mathrm{H} \cdot \mathrm{H}_{2}, \mathrm{H}_{2} \mathrm{O}_{2}\right)
$$

Scheme 4.3

Initial experiments were carried out in argon saturated solutions containing $500 \mathrm{mM}$ ${ }^{t} \mathrm{BuOH}$; ${ }^{t} \mathrm{BuOH}$ scavenges $\bullet \mathrm{OH}$, leaving the solvated electron to reduce the $\left[\mathrm{Co}^{\mathrm{II}}\right]$ catalyst ("electron method," Scheme 4.4). Upon radiolysis, kinetics traces were collected at $20 \mathrm{~nm}$ intervals between 380 and $800 \mathrm{~nm}$, with additional measurements made near the peak maxima and minima of transient absorptions and bleaches, respectively. The absorption differences at $40 \mu$ s were plotted for each sample interval recorded, generating a transient difference spectra (Figure 4.5). At pH 8, a large absorption centered at $606 \mathrm{~nm}$ and a bleach centered at $456 \mathrm{~nm}$ were observed in the difference spectra, indicating that $\left[\mathrm{Co}^{\mathrm{II}}\right]$ was reduced to $\left[\mathbf{C o}^{\mathrm{I}}\right]$. The kinetics traces at $610 \mathrm{~nm}$ were fit to a single exponential, and the observed first-order rate constants obtained are linearly dependent on the concentration of $\left[\mathbf{C o}^{\mathrm{II}}\right]$, giving a second-order rate constant for reduction of $\left[\mathrm{Co}^{\mathrm{II}}\right]$ by $e_{\mathrm{aq}}^{-}$of $4.5 \times 10^{10} \mathrm{M}^{-1} \mathrm{~s}^{-1}$ 
(Figure 4.6). At $\mathrm{pH}$ 4, the transient difference spectrum indicated substantially reduced yield of $\left[\mathbf{C o}^{\mathrm{I}}\right]$, based on the initial dose of solvated electrons, as compared to the data collected at $\mathrm{pH}$ 8. This discrepancy is explained by the reactivity of $e_{\mathrm{aq}}{ }^{-}$with protons to form $\mathrm{H} \bullet$, which occurs with a rate of $2.3 \times 10^{10} \mathrm{M}^{-1} \mathrm{~s}^{-1}$ and competes with the reduction of $\left[\mathbf{C o}^{\mathrm{II}}\right] .{ }^{26}$ This reactivity presents significant challenges for studying the protonation of the $\left[\mathrm{Co}^{\mathrm{I}}\right]$ species; as the concentration of protons is increased, less $\left[\mathrm{Co}^{\mathrm{I}}\right]$ is produced.

$$
\cdot \mathrm{OH}+{ }^{t} \mathrm{BuOH} \longrightarrow \mathrm{H}_{2} \mathrm{O}+0.96\left(\cdot \mathrm{CH}_{2}\right)\left(\mathrm{CH}_{3}\right)_{2} \mathrm{COH}+0.04\left(\mathrm{CH}_{3}\right)_{3} \mathrm{CO} \text {. }
$$

\section{Scheme 4.4}

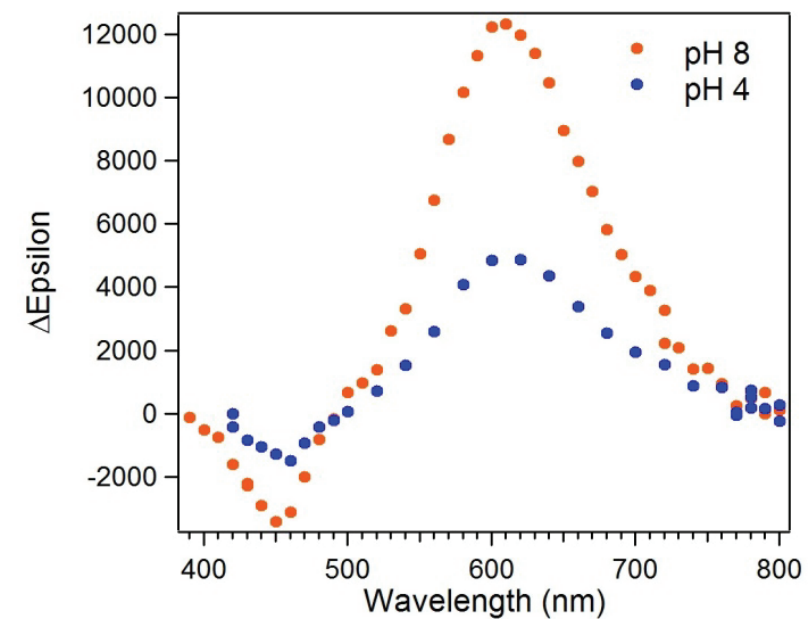

Figure 4.5 Difference spectra for the reaction of $\mathrm{Co}\left(\mathrm{dmgBF}_{2}\right)_{2}\left(\mathrm{H}_{2} \mathrm{O}\right)_{2}$ with $e_{\mathrm{aq}}{ }^{-}$, determined from kinetics traces 40 $\mu$ s after radiolysis pulse at $\mathrm{pH} 4$ and 8 . Conditions: $10 \mathrm{mM}$ $\mathrm{Na}_{2} \mathrm{HPO}_{4}, 500 \mathrm{mM}{ }^{t} \mathrm{BuOH}$, and ca. $45 \mu \mathrm{M} \mathrm{Co}\left(\mathrm{dmgBF}_{2}\right)_{2}\left(\mathrm{H}_{2} \mathrm{O}\right)_{2}$, Ar atmosphere, $\mathrm{pH}$ adjusted with $\mathrm{H}_{2} \mathrm{SO}_{4}$. 


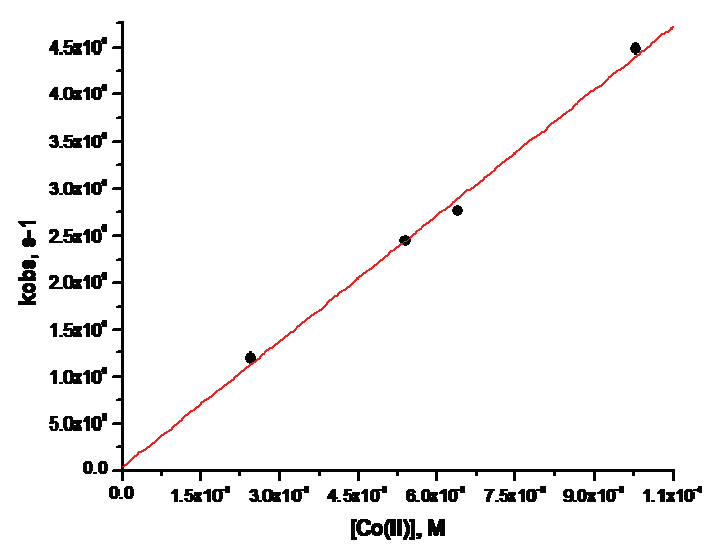

Figure 4.6 Observed first-order rate of $\mathrm{Co}\left(\mathrm{dmgBF}_{2}\right)_{2}\left(\mathrm{H}_{2} \mathrm{O}\right)_{2}$ reduction by $e_{\mathrm{aq}}{ }^{-}$, determined from the bleach of $\mathrm{Co}\left(\mathrm{dmgBF}_{2}\right)_{2}\left(\mathrm{H}_{2} \mathrm{O}\right)_{2}$ signal at $456 \mathrm{~nm}$. Based on the linear relationship of the first-order rate constant to cobaloxime concentration was observed, a second-order rate constant of $4.5 \mathrm{x}$ $10^{10} \mathrm{M}^{-1} \mathrm{~s}^{-1}$ was determined.

To circumvent the challenges associated with $e_{\mathrm{aq}}{ }^{-}$at low $\mathrm{pH}$, a second set of experiments was carried out in solutions containing $10 \mathrm{mM} \mathrm{NaHCO}$ and saturated with $\mathrm{N}_{2} \mathrm{O} ; \mathrm{N}_{2} \mathrm{O}$ scavenges all solvated electrons formed upon ionization to form $\bullet \mathrm{OH}$ (as well as $\mathrm{N}_{2}$ and $\mathrm{OH}^{-}$), which reacts with $\mathrm{HCO}_{2}{ }^{-}$to form the potent reductant, $\mathrm{CO}_{2}{ }^{-}$, capable of transferring an electron to [Co $\left.{ }^{\mathrm{II}}\right]$ ("formate method," Scheme 4.5). This method circumvents problems associated with deleterious proton reactivity, as the electrons are exclusively scavenged by $\mathrm{N}_{2} \mathrm{O}$ and $\mathrm{CO}_{2}{ }^{--}$is not easily protonated. 


$$
\begin{aligned}
e_{\mathrm{aq}}{ }^{-}+\mathrm{CO}_{2} & \longrightarrow \mathrm{CO}_{2}{ }^{--} \\
\mathrm{e}_{\mathrm{aq}}{ }^{-}+\mathrm{N}_{2} \mathrm{O} & \longrightarrow \\
\mathrm{e}_{\mathrm{aq}}{ }^{-}+\mathrm{H}^{+} & \longrightarrow \mathrm{OH}+\mathrm{N}_{2}+\mathrm{OH}^{-} \\
\cdot \mathrm{OH}+\mathrm{HCO}_{2}^{-} & \longrightarrow \mathrm{H} \cdot \\
\mathrm{H} \cdot+\mathrm{HCO}_{2}^{-} \longrightarrow \mathrm{H}_{2} \mathrm{O}+\mathrm{CO}_{2}^{--} & \longrightarrow \mathrm{H}_{2}+\mathrm{CO}_{2}^{--}
\end{aligned}
$$

Scheme 4.5

Similar to experiments with $e_{\mathrm{aq}}{ }^{-}$, kinetics traces were recorded at $20 \mathrm{~nm}$ intervals between 380 and $800 \mathrm{~nm}$ and a transient difference spectrum was constructed at $40 \mu \mathrm{s}$ after radiolysis for samples at $\mathrm{pH} 4$ and 8 . At both $\mathrm{pH} 4$ and 8 , a large absorption centered at 606 $\mathrm{nm}$ and a bleach centered at $456 \mathrm{~nm}$ were observed in the difference spectra, indicating that $\left[\mathrm{Co}^{\mathrm{II}}\right]$ was reduced to $\left[\mathrm{Co}^{\mathrm{I}}\right]$ (Figure 4.7 ). When compared to the difference spectrum obtained via reaction with $e_{\mathrm{aq}}$, the magnitude of the bleach at $456 \mathrm{~nm}$ was identical, yet the absorption centered at $606 \mathrm{~nm}$ was approximately two-thirds the magnitude of that observed in the previous experiment. Kinetics traces were then measured at longer timescales, and a transient difference spectrum was constructed at a $500 \mathrm{~ms}$ time delay, which precisely matched the $40 \mu$ spectrum obtained via reaction with $e_{\mathrm{aq}}^{-}$. Similarly, the reduction could be treated as a pseudo-first-order reaction as the concentration of the cobaloxime was significantly greater than $\mathrm{CO}_{2}{ }^{-}$, and the observed first-order rate constants for the formation of $\left[\mathrm{Co}^{\mathrm{I}}\right]$ obtained from kinetics traces at $610 \mathrm{~nm}$ were plotted as versus the concentration of $\left[\mathrm{Co}^{\mathrm{II}}\right]$ to obtain a second-order rate constant for reduction of [Co $\left.\mathbf{o}^{\mathrm{II}}\right]$, $1.1 \times 10^{9} \mathrm{M}^{-1} \mathrm{~s}^{-1}$ (Figure 4.8). The rate of $\left[\mathrm{Co}^{\mathrm{II}}\right]$ reduction by $\mathrm{CO}_{2}{ }^{--}$is over an order of magnitude slower than that by $e_{\mathrm{aq}}{ }^{-}$. This discrepancy is attributed to the differing mechanisms of reduction. An inner-sphere mechanism for reduction of $\left[\mathrm{Co}^{\mathrm{II}}\right]$ by $\mathrm{CO}_{2}{ }^{-{ }^{-}}$is thought to be responsible for the slow timescale, ${ }^{26}$ though $\mathrm{CO}_{2}$ does not remain bound to 
the reduced species. Samples at lower $\mathrm{pH}$ were also studied in an attempt to study the protonation process; however, the high proton concentration promoted catalyst degradation, complicating the reaction kinetics as the decomposition products also react with the reductants (vide infra).

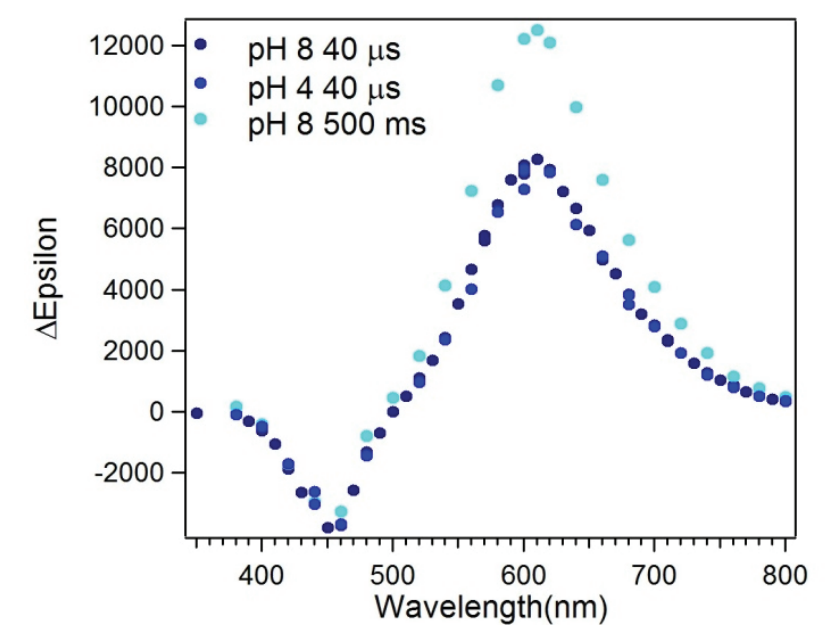

Figure 4.7 Difference spectra for the reaction of $\mathrm{Co}\left(\mathrm{dmgBF}_{2}\right)_{2}\left(\mathrm{H}_{2} \mathrm{O}\right)_{2}$ with $\mathrm{CO}_{2}{ }^{--}$, determined from kinetics traces $40 \mu \mathrm{s}$ or $500 \mathrm{~ms}$ after radiolysis pulse. Conditions: $10 \mathrm{mM}$ $\mathrm{Na}_{2} \mathrm{HPO}_{4}, 10 \mathrm{mM} \mathrm{NaHCO}$, and $\sim 45 \mu \mathrm{M} \mathrm{Co}\left(\mathrm{dmgBF}_{2}\right)_{2}\left(\mathrm{H}_{2} \mathrm{O}\right)_{2}$, $\mathrm{N}_{2} \mathrm{O}$ atmosphere, $\mathrm{pH}$ adjusted with $\mathrm{H}_{2} \mathrm{SO}_{4}$.

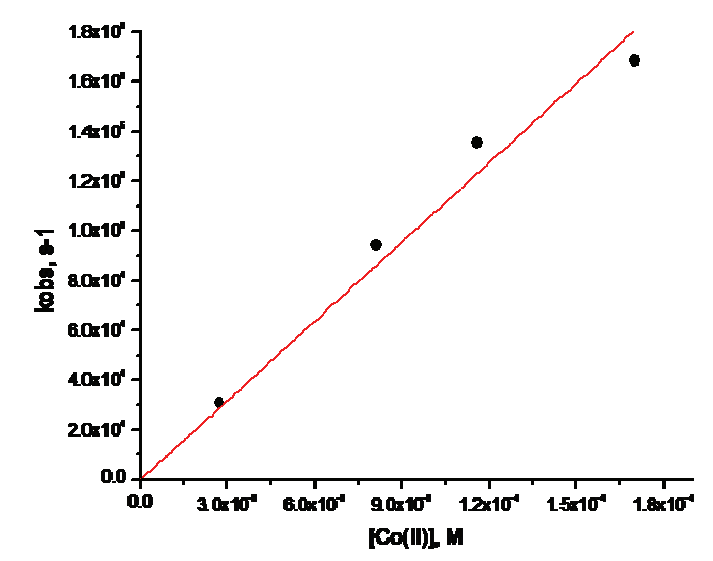

Figure 4.8 Observed first-order rate of $\mathrm{Co}\left(\mathrm{dmgBF}_{2}\right)_{2}\left(\mathrm{H}_{2} \mathrm{O}\right)_{2}$ reduction by $\mathrm{CO}_{2}{ }^{-}$determined from the bleach of $\mathrm{Co}\left(\mathrm{dmgBF}_{2}\right)_{2}\left(\mathrm{H}_{2} \mathrm{O}\right)_{2}$ signal at $456 \mathrm{~nm}$. Based on the linear relationship of the first-order rate constant to cobaloxime concentration was observed, a second-order rate constant of $1.1 \mathrm{x}$ $10^{9} \mathrm{M}^{-1} \mathrm{~s}^{-1}$ was determined. 
A third scavenging technique was employed, in an attempt to observe the [Co $\left.{ }^{\mathrm{III}} \mathbf{H}\right]$ spectrum. In solutions containing $500 \mathrm{mM}$ t $\mathrm{BuOH}$ and $0.3 \mathrm{M} \mathrm{H}_{2} \mathrm{SO}_{4}$ under an argon atmosphere, $\mathrm{H} \bullet$ is quantitatively formed from $e_{\mathrm{aq}}{ }^{-}$upon radiolysis, along with $\bullet^{t} \mathrm{BuOH}\left({ }^{\text {" }} \mathrm{H} \bullet\right.$ method," Scheme 4.6).

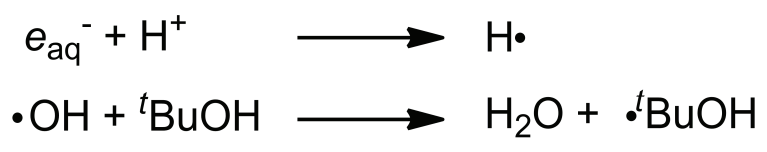

Scheme 4.6

Motivated by the reactivity of $\mathrm{N}$-meso-CoL ${ }^{2+}(\mathrm{L}=5,7,7,12,14,14$-hexamethyl-1,4,8,11tetraazacyclotetradeca-4,11-diene) with $\mathrm{H} \bullet$ to form the corresponding $\mathrm{Co}^{\mathrm{III}} \mathrm{H}$ species, we aimed to observe similar reactivity with $\left[\mathrm{Co}^{\mathrm{II}}\right] .{ }^{26}$ However, attempts to observe the resulting spectrum were met with challenges arising from the decomposition of $\left[\mathbf{C o}^{\mathrm{II}}\right]$ at such a high acid concentration. In response, a rapid mix technique was employed wherein two sample mixtures, one containing $1 \mathrm{M}{ }^{t} \mathrm{BuOH}$ and $180 \mu \mathrm{M}\left[\mathrm{Co}^{\mathrm{II}}\right]$ and the second containing $0.6 \mathrm{M} \mathrm{H}_{2} \mathrm{SO}_{4}$, were rapidly combined in a mixing chamber before being injected to the sample observation chamber. With the pulse occurring within 1 second of mixing, negligible decomposition occurs before detection. The difference spectrum obtained at 5 ms shows a bleach at $456 \mathrm{~nm}$ and a new absorption at $300 \mathrm{~nm}$. The absolute spectrum shows a peak shoulder at $350 \mathrm{~nm}$ with a tail into the visible region (Figure 4.9). The spectrum was tentatively assigned to $\left[\mathrm{Co}^{\mathrm{III}} \mathbf{H}\right]$, as the absorption spectrum displayed similar features to the isolable $\mathrm{MeCo}\left(\mathrm{dmgBF}_{2}\right)_{2}$, which has a peak at $370 \mathrm{~nm}$ trailing into the visible region. However, the features in the deep UV were unresolved and discrepancies between molar extinction coefficients appeared during data analysis. Further, questions arose about 
the potential reactivity of $\left[\mathrm{Co}^{\mathrm{II}}\right]$ with ${ }^{\bullet} \mathrm{BuOH}$; reaction of this radical with $\mathrm{N}-\mathrm{rac}$ - $\mathrm{CoL}^{2+}$ was observed in previous studies $\left(k=1.4 \times 10^{6} \mathrm{M}^{-1} \mathrm{~s}^{-1}\right){ }^{26}$

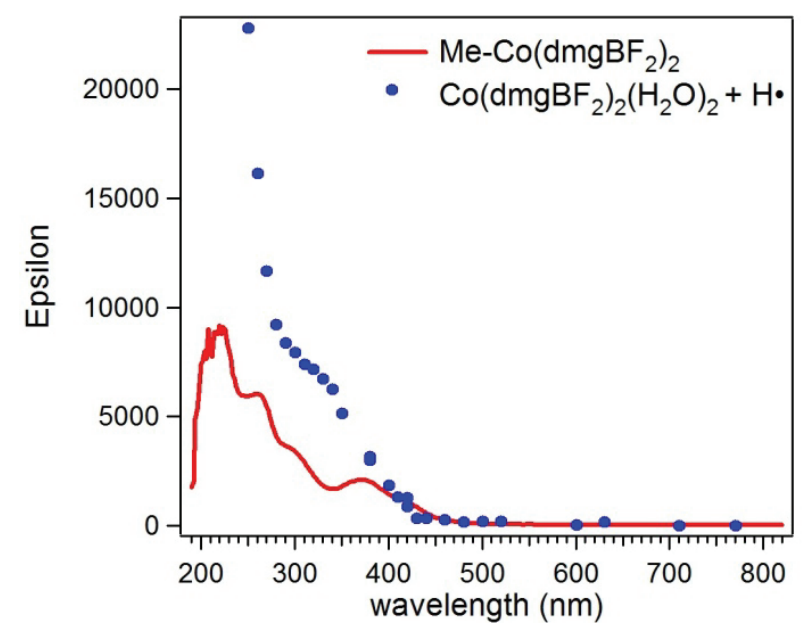

Figure 4.9 Absolute spectrum of $\mathrm{Co}\left(\mathrm{dmgBF}_{2}\right)_{2}\left(\mathrm{H}_{2} \mathrm{O}\right)_{2}+\mathrm{H}$ • obtained from kinetics traces $40 \mu$ after radiolysis pulse, obtained via the "H• method" and overlaid with the absorption spectrum of $\mathrm{MeCo}\left(\mathrm{dmgBF}_{2}\right)_{2}$ in acetonitrile. Conditions: $0.5 \mathrm{M}{ }^{t} \mathrm{BuOH}, 0.3 \mathrm{M}$ $\mathrm{H}_{2} \mathrm{SO}_{4}$, and $90 \mu \mathrm{M} \mathrm{Co}\left(\mathrm{dmgBF}_{2}\right)_{2}\left(\mathrm{H}_{2} \mathrm{O}\right)_{2}$, Ar atmosphere, prepared via rapid mixing.

To further probe the potential reactivity of $\left[\mathrm{Co}^{\mathrm{II}}\right]$ with $\bullet^{t} \mathrm{BuOH}, \mathrm{N}_{2} \mathrm{O}$ sparged solutions containing ${ }^{t} \mathrm{BuOH}$ and $\left[\mathrm{Co}^{\mathrm{II}}\right]$ at $\mathrm{pH} 8$ were examined. Under these conditions, radiolysis should primarily yield $\bullet^{t} \mathrm{BuOH}$ (Scheme 4.7 ). As indicated by the kinetics trace at $465 \mathrm{~nm}$, $\left[\mathbf{C o}^{\mathrm{II}}\right]$ is consumed upon reaction with ${ }^{t} \mathrm{BuOH}$ to form an alkyl adduct, $\left[\mathbf{C o}^{\mathrm{III}} \mathbf{R}\right]$. The kinetics trace was analyzed, accounting for the self-recombination pathways of $\bullet^{t} \mathrm{BuOH}(k=$ $\left.1.05 \times 10^{9} \mathrm{M}^{-1} \mathrm{~s}^{-1}\right),{ }^{27}$ to obtain a second-order rate constant for the reaction of [Co $\left.{ }^{\mathrm{II}}\right]$ with ${ }^{-t} \mathrm{BuOH}\left(k=3.5 \times 10^{7} \mathrm{M}^{-1} \mathrm{~s}^{-1}\right.$, Figure 4.10$)$. Based on radiolysis yields, $4.5 \mu \mathrm{M}$ of $\bullet^{t} \mathrm{BuOH}$ were produced, yet only $1.5 \mu \mathrm{M}$ of $\left[\mathbf{C o}^{\mathrm{II}}\right]$ are consumed, indicating the other two-thirds of ${ }^{t} \mathrm{BuOH}$ self reacted. Considering the reaction yield of ${ }^{t} \mathrm{BuOH}$ with $\left[\mathbf{C o}^{\mathrm{II}}\right]$, a transient absolute spectrum of [C $\left.\mathbf{C o}^{\mathrm{III}} \mathbf{R}\right]$ was calculated (Figure 4.11). Absorption features similar to 
those seen in traces obtained via the " $\mathrm{H} \bullet$ method" are observed. Based on the experimentally determined reactivity of ${ }^{t} \mathrm{BuOH}$, the spectrum of this adduct could be subtracted from the "H• method" spectrum to obtain an absorption profile of the hydride, though to date, difficulties in data analysis have prevented this.

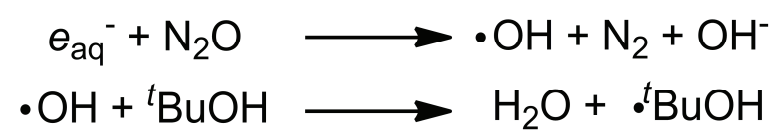

Scheme 4.7

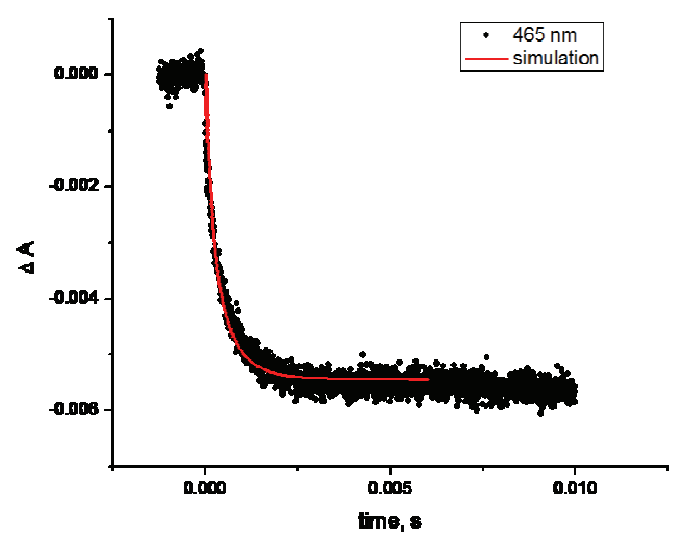

Figure 4.10 Kinetics trace and simulation observed at $465 \mathrm{~nm}$ for the reaction of $\mathrm{Co}\left(\mathrm{dmgBF}_{2}\right)_{2}\left(\mathrm{H}_{2} \mathrm{O}\right)_{2}+\bullet^{t} \mathrm{BuOH}$. Simulation accounts for self-recombination of $\bullet^{t} \mathrm{BuOH}\left(k=1.05 \times 10^{9} \mathrm{M}^{-1} \mathrm{~s}^{-1}\right)$; second-order rate constant for reaction of $\mathrm{Co}\left(\mathrm{dmgBF}_{2}\right)_{2}\left(\mathrm{H}_{2} \mathrm{O}\right)_{2}+$ ${ }^{\cdot} \mathrm{BuOH}$ was determined to be $3.5 \times 10^{7} \mathrm{M}^{-1} \mathrm{~s}^{-1}$. Conditions: 10 $\mathrm{mM} \mathrm{Na}_{2} \mathrm{HPO}_{4}, 500 \mathrm{mM}{ }^{t} \mathrm{BuOH}$ and $50 \mu \mathrm{M} \mathrm{Co}\left(\mathrm{dmgBF}_{2}\right)_{2}\left(\mathrm{H}_{2} \mathrm{O}\right)_{2}$, $\mathrm{N}_{2} \mathrm{O}$ atmosphere, $\mathrm{pH} 8$. 


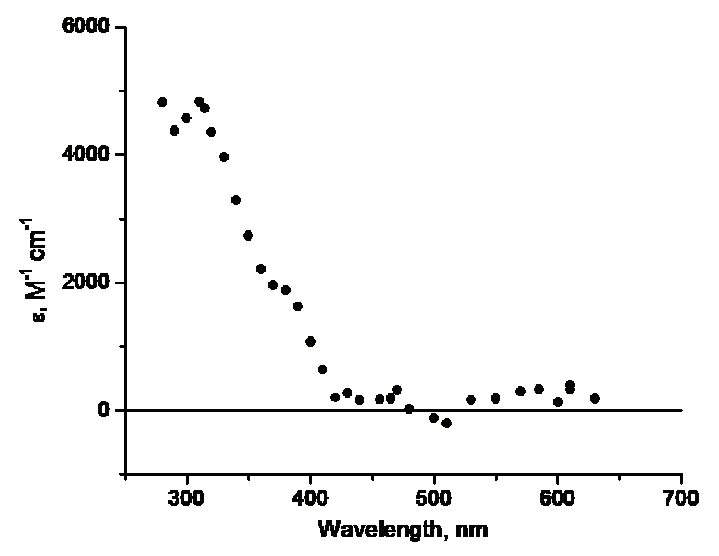

Figure 4.11 Absolute spectrum of $\mathrm{Co}\left(\mathrm{dmgBF}_{2}\right)_{2}\left(\mathrm{H}_{2} \mathrm{O}\right)_{2}+\cdot^{t} \mathrm{BuOH}$ obtained from kinetics traces $\sim 3 \mathrm{~ms}$ after radiolysis pulse. Conditions: $10 \mathrm{mM} \mathrm{Na} \mathrm{HPO}_{4}, 500 \mathrm{mM}{ }^{t} \mathrm{BuOH}$ and $50 \mu \mathrm{M}$ $\mathrm{Co}\left(\mathrm{dmgBF}_{2}\right)_{2}\left(\mathrm{H}_{2} \mathrm{O}\right)_{2}, \mathrm{~N}_{2} \mathrm{O}$ atmosphere, $\mathrm{pH} 8$.

\section{Challenges in Aqueous Mechanistic Studies}

Ultimately, mechanistic investigations in aqueous conditions are thwarted by issues associated with catalyst degradation. Berben and Peters noted that Faradaic yields from bulk electrolysis experiments at $\mathrm{pH} 4$ were limited to $15 \%$, and attributed the incomplete production of hydrogen to catalyst degradation. ${ }^{10}$ Here, catalyst stability studies were carried out at several pHs, and while no substantial decomposition was observed in pure water, significant degradation occurred in both acidic and basic buffered solutions. In many pulse radiolysis experiments, sample aliquots were pulsed up to three times and the initial pulse was usually discarded to account for reaction of reductants with degradation impurities, which were usually consumed with the first pulse. At short timescales, the second and third pulses were usually reproducible, but at longer timescales the pulse-topulse reproducibility became a significant issue. Experimental limitations prevented similar impurity removal with photoionization methods. Further, the photo- or radiation-triggered 
generation of reducing equivalents, like $e_{\mathrm{aq}}{ }^{-}$, is typically limited to concentrations on the $\mu \mathrm{M}$ scale, and degradation induced impurities influence reaction kinetics significantly at these concentrations

\section{Summary}

Photo- and radiation-triggered generation of solvated electrons (and other reducing equivalents) has been utilized to reduce $\left[\mathbf{C o}^{\mathrm{II}}\right]$ to $\left[\mathbf{C o}^{\mathrm{I}}\right]$, and transient optical measurements have provided kinetics details about the electron transfer process. Yet while photoionization and pulse radiolysis methods have shown that solvated electrons can rapidly reduce $\left[\mathbf{C o}^{\mathrm{II}}\right]$ to $\left[\mathbf{C o}^{\mathrm{I}}\right], e_{\mathrm{aq}}^{-}$generated at low $\mathrm{pH}$ are scavenged by protons, complicating the $\left[\mathbf{C o}^{\mathrm{I}}\right]$ protonation and $\left[\mathbf{C o}^{\mathrm{III}} \mathbf{H}\right]$ reactivity studies of interest. Further complications arise from acid-catalyzed degradation of the cobaloxime, yielding impurities that significantly influence the reaction kinetics. Ultimately, practical application of these unstable catalysts in a homogeneous system is unlikely, suggesting no further need to pursue these studies. However, work by Berben and Peters has suggested that surface immobilization of catalysts on electrodes leads to active hydrogen evolution in aqueous solutions at low overpotentials, and future mechanistic work should focus on heterogeneous systems.

\section{Acknowledgement}

The pulse radiolysis data reported here was collected at Brookhaven National Laboratory with assistance from Dr. Dmitry Polyanskiy and Dr. Etsuko Fujita. This work was supported by the NSF Center for Chemical Innovation (Powering the Planet, CHE0802907, CHE-0947829), the Arnold and Mabel Beckman Foundation, CCSER (Gordon

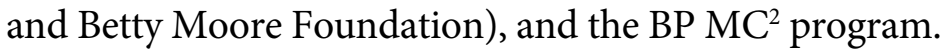




\section{Experimental Details}

\section{General}

All materials, unless noted, were used as received. $\mathrm{Co}\left(\mathrm{dmgBF}_{2}\right)\left(\mathrm{H}_{2} \mathrm{O}\right)_{2}$ was prepared according to the method of Bakac and Espenson. ${ }^{28} \mathrm{UV}$-visible absorption measurements were carried out using a Hewlett Packard 8452 UV-Vis spectrophotometer in $1 \mathrm{~cm}$ pathlength quartz cuvettes.

\section{Photoionization experiments}

Time-resolved spectroscopic measurements were carried out at the Beckman Institute Laser Resource Center. Laser excitation was provided by 8 ns pulses from a $10 \mathrm{~Hz}$ Q-switched Nd:YAG laser (Spectra-Physics Quanta-Ray PRO-Series). The third harmonic was used directly for NADH experiments $\left(\lambda_{\mathrm{ex}}=355 \mathrm{~nm}\right)$, while the fourth harmonic was used for indole experiments $\left(\lambda_{\text {ex }}=266 \mathrm{~nm}\right)$. Probe light for transient absorption kinetics measurements was provided by a $75 \mathrm{~W}$ arc lamp (PTI Model A 1010) that could be operated in continuous wave or pulsed modes. Timing between the laser and the probe light was controlled by a digital delay generator (EG\&G 9650). After passing through the sample collinearly with the laser beam, scattered excitation light was rejected by suitable long pass and short pass filters, and probe wavelengths were selected for detection by a double monochromator (Instruments SA DH-10) with $1 \mathrm{~mm}$ slits. Transmitted light was detected with a photomultiplier tube (PMT, Hamamatsu R928). The PMT current was amplified and recorded with a transient digitizer (LeCroy 9354A or Tektronix DSA 602). Absorption data were averaged over at least 500 laser pulses. The data were converted to units of $\Delta \mathrm{OD}\left(\Delta \mathrm{OD}=-\log _{10}\left(\mathrm{I} / \mathrm{I}_{0}\right)\right.$, where $\mathrm{I}$ is the time-resolved probe-light intensity with laser excitation, and $\mathrm{I}_{0}$ is the intensity without excitation). 
Probe light for transient absorption measurements was provided by white light flash lamp sources with either nanosecond or microsecond durations. Probe light was transported via a fiber optic and split by a partial reflector. Approximately $70 \%$ of the probe light was passed through the sample, with the remainder directed around the sample as a reference beam. Sample excitation by the laser beam was collinear with the probe light. Light intensity was read by two photodiode arrays (Ocean Optics S1024DW Deep Well Spectrometer), with scattered excitation light rejected by a long pass filter. The timing synchronization of the laser fire, flashlamp fire, and photodiode array readout was controlled by a series of timing circuits triggered by either a laser lamp sync pulse. The photodiode readout was interfaced with a PC via a National Instruments multifunction input/output card. Measurements were made with and without excitation, and corrected for background light. Difference spectra were averaged over approximately 500 shots.

All instruments and electronics in these systems were controlled by software written in LabVIEW (National Instruments). Data manipulation was performed with MATLAB R2008a (Mathworks, Inc.) and graphed with Igor Pro 5.01 (Wavemetrics).

\section{Photoionization Sample Preparations:}

$\mathrm{NADH}$

Typical solutions contained $300-1000 \mu \mathrm{M} \mathrm{NADH}$ and $100-150 \mu \mathrm{M} \mathrm{Co}\left(\mathrm{dmgBF}_{2}\right)_{2}\left(\mathrm{H}_{2} \mathrm{O}\right)_{2}$ in $10 \mathrm{mM} \mathrm{KPi}$ buffer. Solutions were degassed through repeated pump-purge cycles with argon. 


\section{Indole}

Indole was recrystallized prior to use. Solutions were heated to moderate temperatures (35$40{ }^{\circ} \mathrm{C}$ ) until the indole dissolved. Typical samples contained $1 \mathrm{mM}$ indole, $10 \mathrm{mM} \mathrm{KP} i$ buffer with $100 \mathrm{mM}$ ionic strength adjusted with $\mathrm{Na}_{2} \mathrm{SO}_{4}$, and $\sim 150 \mu \mathrm{M}$ $\mathrm{Co}\left(\mathrm{dmgBF}_{2}\right)_{2}\left(\mathrm{H}_{2} \mathrm{O}\right)_{2}$. Solutions were degassed through repeated pump-purge cycles with argon.

\section{Pulse Radiolysis Experiments}

Pulse radiolysis transient absorption experiments were carried out with a $2 \mathrm{MeV}$ Van de Graaff accelerator at Brookhaven National Laboratory. Pulse lengths were typically $0.2-3$ $\mu$ s. Except for experiments collected via the $\mathrm{H} \bullet$ method, samples were degassed in a quartz pulse-radiolysis cell consisting of a $50 \mathrm{~mL}$ reservoir that drains into a $2 \mathrm{~cm} \mathrm{x} 1 \mathrm{~cm}$ x $0.5 \mathrm{~cm}$ cell. The samples were irradiated through the $0.5 \mathrm{~cm}$ dimension and probe light was passed through a $2 \mathrm{~cm}$ pathlength. At short timescales $(<100 \mu \mathrm{s})$, a Xe arc lamp was utilized, while a Tungsten lamp provided probe light for other experiments. ${ }^{29}$ After passing through the sample, probe wavelengths were selected for detection by a double monochromator. Transmitted light was detected with a photomultiplier tube and data was recorded by a PCcontrolled CAMAC-based data acquisition and control system. Irradiated solution aliquots were drained from the cell after 1,2, or 3 shots, as noted, and the sample chamber was filled with fresh solution. Difference spectra were scaled with a dosimetry correction. Absolute spectra were calculated from difference spectra by adding the absorption of the $\mathrm{Co}\left(\mathrm{dmgBF}_{2}\right)_{2}\left(\mathrm{H}_{2} \mathrm{O}\right)_{2}$. 
Pulse Radiolysis Sample Preparation.

\section{Electron Method}

Samples contained $10 \mathrm{mM} \mathrm{Na}_{2} \mathrm{HPO}_{4}$ and were adjusted to the desired $\mathrm{pH}$ with $\mathrm{H}_{2} \mathrm{SO}_{4}, 500$ $\mathrm{mM}{ }^{t} \mathrm{BuOH}$ and $\sim 40 \mu \mathrm{M} \mathrm{Co}\left(\mathrm{dmgBF}_{2}\right)_{2}\left(\mathrm{H}_{2} \mathrm{O}\right)_{2}$ and were sparged with argon for at least 20 minutes. Each sample aliquot was radiolyzed three times; the first sample was discarded while data from the second and third shots were utilized.

\section{Formate Method}

Samples contained $10 \mathrm{mM} \mathrm{Na}_{2} \mathrm{HPO}_{4}$ and were adjusted to the desired $\mathrm{pH}$ with $\mathrm{H}_{2} \mathrm{SO}_{4}, 10$ mM NaHCO 2 , and $\sim 40 \mu \mathrm{M} \mathrm{Co}\left(\mathrm{dmgBF}_{2}\right)_{2}\left(\mathrm{H}_{2} \mathrm{O}\right)_{2}$ and were sparged with $\mathrm{N}_{2} \mathrm{O}$ for at least 20 minutes. Each sample aliquot was radiolyzed three times; the first sample was discarded while data from the second and third shots were utilized.

\section{H• Method}

Samples were prepared via rapid mixing to minimize acid catalyzed cobaloxime degradation. One syringe was filled with a solution containing $1 \mathrm{M}{ }^{t} \mathrm{BuOH}$ and $180 \mu \mathrm{M}$ $\mathrm{Co}(\mathrm{dmgBF})_{2}\left(\mathrm{H}_{2} \mathrm{O}\right)_{2}$ and a second syringe was filled with $0.6 \mathrm{M} \mathrm{H}_{2} \mathrm{SO}_{4}$. Syringe volumes were sparged with argon for at least 20 minutes. Equal aliquots of each were injected into a mixing chamber via a computer controlled syringe pump and the sample was radiolyzed within 1 second of mixing. Each sample was pulsed only once.

\section{${ }^{t} \mathrm{BuOH}$ Method}

Samples contained $10 \mathrm{mM} \mathrm{Na}_{2} \mathrm{HPO}_{4}, 500 \mathrm{mM}{ }^{t} \mathrm{BuOH}$ and $50 \mu \mathrm{M} \mathrm{Co}\left(\mathrm{dmgBF}_{2}\right)_{2}\left(\mathrm{H}_{2} \mathrm{O}\right)_{2}$ at pH 8 and were sparged with $\mathrm{N}_{2} \mathrm{O}$ atmosphere for at least 20 minutes. 


\section{References}

1. Karunadasa, H. I.; Chang, C. J.; Long, J. R. Nature 2010, 464, 1329.

2. Collin, J. P.; Jouaiti, A.; Sauvage, J. P. Inorg. Chem. 1988, 27, 1986.

3. Krishnan, C. V.; Sutin, N. J. Am. Chem. Soc. 1981, 103, 2141.

4. Kellett, R. M.; Spiro, T. G. Inorg. Chem. 1985, 24, 2373.

5. Endicott, J. F.; Rillema, D. P.; Papaconstantinou, E. Inorg. Chem. 1971, 10, 1739.

6. Tait, A. M.; Hoffman, M. Z.; Hayon, E. J. Am. Chem. Soc. 1976, 98, 86.

7. Brown, G. M.; Brunschwig, B. S.; Creutz, C.; Endicott, J. F.; Sutin, N. J. Am. Chem. Soc. $1979,101,1298$.

8. Connolly, P.; Espenson, J. H. Inorg. Chem. 1986, 25, 2684.

9. Dempsey, J. L.; Brunschwig, B. S.; Winkler, J. R.; Gray, H. B. Acc. Chem. Res. 2009, 42, 1995.

10. Berben, L. A.; Peters, J. C. Chem. Commun. 2010, 46, 398.

11. Hu, X.; Brunschwig, B. S.; Peters, J. C. J. Am. Chem. Soc. 2007, 129, 8988.

12. Hu, X. L.; Cossairt, B. M.; Brunschwig, B. S.; Lewis, N. S.; Peters, J. C. Chem. Commun. $2005,4723$.

13. Baffert, C.; Artero, V.; Fontecave, M. Inorg. Chem. 2007, 46, 1817.

14. Buxton, G. V.; Sellers, R. M. Coord. Chem. Rev. 1977, 22, 195.

15. Orii, Y. Biochemistry 1993, 32, 11910.

16. Czochralska, B.; Lindqvist, L. Chem. Phys. Lett. 1983, 101, 297.

17. Wittung-Stafshede, P.; Malmstrom, B. G.; Winkler, J. R.; Gray, H. B. J. Phys. Chem. A 1998, 102, 5599.

18. Telford, J. R.; Tezcan, F. A.; Gray, H. B.; Winkler, J. R. Biochemistry 1999, 38, 1944.

19. Lee, J. C.; Gray, H. B.; Winkler, J. R. Proc. Natl. Acad. Sci. U. S. A. 2001, 98, 7760. 
20. Braun, R. D.; Santhanam, K. S. V.; Elving, P. J. J. Am. Chem. Soc. 1975, 97, 2591.

21. Anderson, A. G.; Berkelhammer, G. J. Am. Chem. Soc. 1958, 80, 992.

22. Johnston, C. C.; Gardner, J. L.; Suelter, C. H.; Metzler, D. E. Biochemistry 1963, 2, 689.

23. Katoh, R. J. Photochem. Photobiol. A 2007, 189, 211.

24. Baugher, J. F.; Grossweiner, L. I. J. Phys. Chem. 1977, 81, 1349.

25. Stevenson, K. L.; Papadantonakis, G. A.; LeBreton, P. R. J. Photochem. Photobiol. A 2000, 133, 159.

26. Creutz, C.; Schwarz, H. A.; Wishart, J. F.; Fujita, E.; Sutin, N. J. Am. Chem. Soc. 1991, 113,3361 .

27. Mezyk, S. P.; Madden, K. P. J. Phys. Chem. A 1998, 103, 235.

28. Bakac, A.; Espenson, J. H. J. Am. Chem. Soc. 1984, 106, 5197.

29. Schwarz, H. A.; Creutz, C. Inorg. Chem. 1983, 22, 707. 
Chapter 5

\section{CATALYTIC $\mathrm{H}_{2}$ EVOLUTION FROM A BINUCLEAR COBALOXIME}

\section{Portions of this work are excerpted with permission from:}

Valdez, C. N.; Dempsey, J. L.; Brunschwig, B. S.; Winkler, J. R.; Gray, H. B. Submitted. Unpublished Material Copyright 2010 American Chemical Society 


\section{Introduction}

Efficient catalytic reduction of protons to dihydrogen is necessary for one half of a functioning solar water splitting system. Significant progress has been made in developing and understanding catalysts capable of producing hydrogen from acidic media, using either small molecule mimics of hydrogenase active sites or other synthetic systems. Difluoroboryl-bridged $\mathrm{Co}^{\mathrm{II}}$-diglyoxime complexes have been shown to catalyze hydrogen evolution at low overpotentials. ${ }^{1}$ With these complexes, hydrogen evolution is initiated upon reduction to $\mathrm{Co}^{\mathrm{I}}$ which is believed to react with a proton donor to form a $\mathrm{Co}^{\mathrm{III}}$ hydride $\left(\mathrm{Co}^{\mathrm{III}} \mathrm{H}\right)$. The $\mathrm{Co}^{\mathrm{III}} \mathrm{H}$ intermediate can either undergo subsequent protonation to release $\mathrm{H}_{2}$ and generate a Co ${ }^{\mathrm{III}}$ species that is reduced to regenerate the catalyst (heterolytic route), or it can react in a bimolecular fashion with another $\mathrm{Co}^{\mathrm{III}} \mathrm{H}$ to eliminate $\mathrm{H}_{2}$ (homolytic route). Alternatively, $\mathrm{Co}^{\mathrm{III}} \mathrm{H}$ can be reduced further to form $\mathrm{Co}^{\mathrm{II}} \mathrm{H}$, which can react via similar heterolytic or homolytic routes. Digital simulations of electrocatalytic waves performed by $\mathrm{Hu}$ et al. indicated that bimetallic reactivity of two $\mathrm{Co}^{\mathrm{III}}$ hydrides is predominately responsible for hydrogen evolution and our recent electron transfer studies (reported in Chapter 2) revealed that the barriers to $\mathrm{H}_{2}$ evolution associated with this pathway are more favorable than those for the protonation of a $\mathrm{Co}^{\mathrm{III}} \mathrm{H}^{2-3}$ The work presented in Chapter 3 suggests that under conditions in which $\mathrm{Co}^{\mathrm{III}} \mathrm{H}$ is present in low concentrations and an excess of reducing equivalents are available, the hydrogen evolution pathway via protonation of $\mathrm{Co}^{\mathrm{II}} \mathrm{H}$ is favored. ${ }^{4}$

In the low-barrier homolytic pathway, two $\mathrm{Co}^{\mathrm{III}} \mathrm{H}$ species must diffuse together in solution in order to react and release $\mathrm{H}_{2}$. While findings suggest that the rate-limiting step for hydrogen evolution may be hydride formation, ${ }^{2}$ immobilization onto an electrode surface, as may be required in an efficient water splitting device, might inhibit the bimolecular 
pathway and decrease the device's efficiency. ${ }^{5-7}$ Binuclear analogues of many catalysts that rely on bimetallic cooperative activity show enhanced activity when compared to the monomeric species, including certain $\mathrm{Ru}$ and Os diporphyrin $\mathrm{H}_{2}$ evolving catalysts with cofacial orientation..$^{8-9}$ In one noteworthy example, Jones and coworkers showed that a "bisalen" analogue of Jacobsen's Co-(salen) catalyst for hydrolytic kinetic resolution of racemic epoxides (a reaction that also shows a second-order kinetic dependence on catalyst) maintained activity when linked to a solid support, while function of the analogous monomer was essentially shut off when immobilized. ${ }^{10-12}$ Thus, with a cobaloxime modified electrode in mind and an interest in potentially increasing catalytic $\mathrm{H}_{2}$ evolution rates, we synthesized and electrochemically characterized a covalently linked dicobalt catalyst.

\section{Results and Discussion}

The synthesis for an octamethylene-linked bis(glyoxime) ligand, tetradecane-2,3,12,13tetraone tetraoxime $\left(\mathrm{LH}_{4}\right)$, was adapted from the procedure of Busch and coworkers (Scheme 5.1). ${ }^{13}$ Reaction of the ligand with 2 eq. diphenylglyoxime and 2 eq. cobalt(II) dichloride in ethanol, followed by addition of 4 eq. pyridine and air as an oxidant yielded a mixture of products, including the desired binuclear $\mathrm{Co}^{\mathrm{III}} \mathrm{Cl}$ complex 5.1, mononuclear $\mathrm{ClCo}^{\mathrm{III}}(\mathrm{dpgH})_{2} \mathrm{py}$, and the singly metalated ligand $\mathrm{ClCo}^{\mathrm{III}}(\mathrm{dpgH})\left(\mathrm{LH}_{3}\right) \mathrm{py}\left(\operatorname{dpgH} \mathrm{H}_{2}=\right.$ diphenylglyoxime, py $=$ pyridine, Scheme 5.2). Product purification issues prevented us from isolating the analogous binuclear species with a dimethylglyoxime ligand.

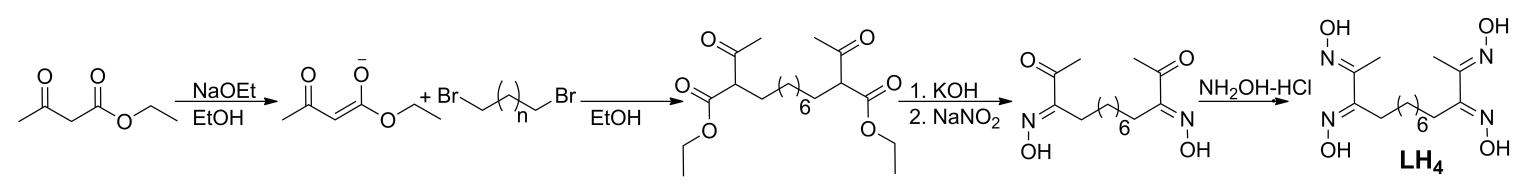

Scheme 5.1 
The reaction mixture was separated via column chromatography and $\mathbf{5 . 1}$ was isolated in $10 \%$ yield. The oxime-linking hydrogen atoms of 5.1 were replaced by bridging $\mathrm{BF}_{2}$ groups, which have been shown to engender enhanced coordination stability and positively shift the reduction potentials of the resulting complexes ${ }^{2}$ by reflux with excess boron trifluoride in acetonitrile (Scheme 5.2). Under these conditions, the axial pyridine was displaced by acetonitrile, yielding the desired binuclear catalyst 5.2.

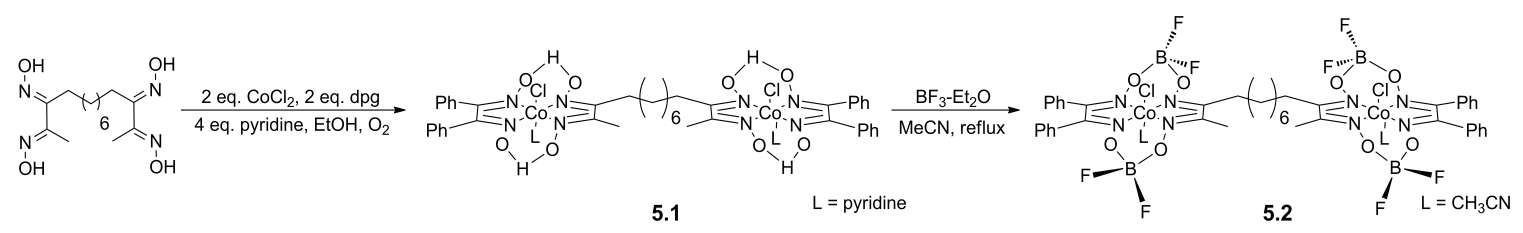

Scheme 5.2

$\mathrm{ClCo}(\mathrm{dmgH})(\mathrm{dpgH})$ py was synthesized by the method of Gupta, and reaction with boron trifluoride diethyl etherate provided $\mathrm{ClCo}\left(\mathrm{dmgBF}_{2}\right)\left(\operatorname{dpgBF}_{2}\right)\left(\mathrm{CH}_{3} \mathrm{CN}\right), \quad \mathbf{5 . 3}$, as a mononuclear analogue of $5.2\left(\mathrm{dmgH}_{2}=\right.$ dimethylglyoxime, Figure 5.1) ${ }^{14}$

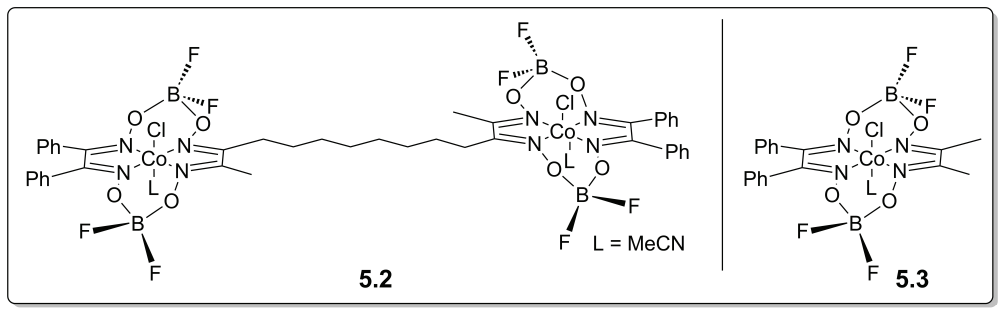

Figure 5.1 Binuclear (5.2) and mononuclear (5.3) cobaloximes studied as hydrogen evolving catalysts.

The cyclic voltammogram of $\mathbf{5 . 2}$ (Figure 5.2) measured in $0.1 \mathrm{M}\left[{ }^{\mathrm{n}} \mathrm{Bu}_{4} \mathrm{~N}\right]\left[\mathrm{PF}_{6}\right]$ acetonitrile solution revealed an irreversible reduction at $\sim 0.2 \mathrm{~V}$ vs. SCE, attributed to the $\mathrm{Co}^{\mathrm{III} / \mathrm{II}}$ reduction accompanied by the loss of an axial chloride, and a reversible redox process at 
$-0.37 \mathrm{~V}$ vs. SCE, assigned to the $\mathrm{Co}^{\mathrm{II} / \mathrm{I}}$ couple. Electrochemical measurements of 5.3 under similar conditions also revealed an irreversible $\mathrm{Co}^{\mathrm{III} / \mathrm{II}}$ couple at $\sim 0.2 \mathrm{~V}$ vs. SCE and the $\mathrm{Co}^{\mathrm{II} / \mathrm{I}}$ couple at $-0.37 \mathrm{~V}$ vs. SCE (Figure 5.3). The identical $\mathrm{Co}^{\mathrm{II} / \mathrm{I}}$ reduction potentials for 5.2 and 5.3 are due to the similarity in electronic properties between the respective ligands. The methyl and alkyl substituents of dimethylglyoxime and tetradecane-2,3,12,13-tetraone tetraoxime have similar electron-donating properties, and the redox potentials the mixed dioxime complexes 2 and 3 lie, unsurprisingly, in between the corresponding $\mathrm{Co}^{\mathrm{II} / \mathrm{I}}$ potentials of $\mathrm{Co}\left(\mathrm{dpgBF}_{2}\right)_{2}\left(\mathrm{CH}_{3} \mathrm{CN}\right)_{2}(\mathbf{2})$ and $\mathrm{Co}\left(\mathrm{dmgBF}_{2}\right)_{2}\left(\mathrm{CH}_{3} \mathrm{CN}\right)_{2}(\mathbf{1})$, which appear at -0.28 and $-0.55 \mathrm{~V}$ vs. SCE, respectively. The $\mathrm{Co}^{\mathrm{II} / \mathrm{I}}$ reductions of both 5.2 and 5.3 are welldefined and reversible with peak separations of approximately $70 \mathrm{mV}$, consistent with a reversible one-electron transfer process at each metal site and suggesting no electronic communication between the two cobalt centers. ${ }^{15}$

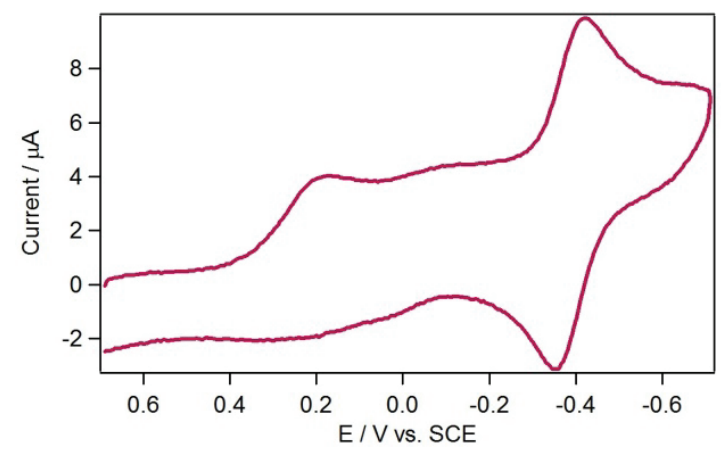

Figure 5.2 Cyclic voltammogram of $0.372 \mathrm{mM} 5.2$ in $0.1 \mathrm{M}$ $\left[\mathrm{NBu}_{4}\right]\left[\mathrm{PF}_{6}\right] \mathrm{CH}_{3} \mathrm{CN}$ solution. Scan rate $=100 \mathrm{mV} / \mathrm{s}$. 


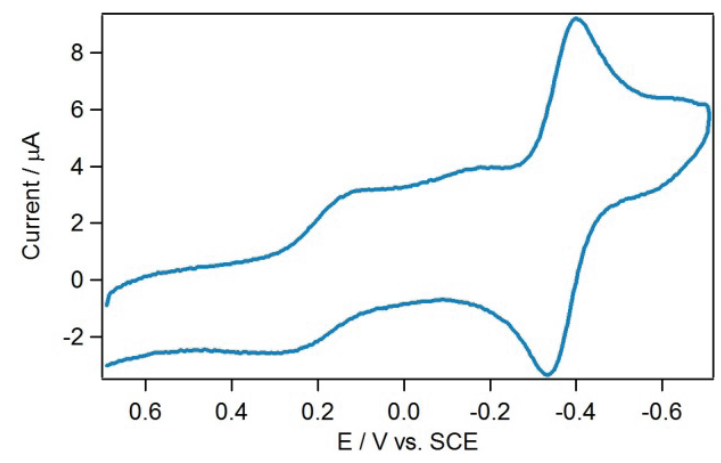

Figure 5.3 Cyclic voltammogram of $0.649 \mathrm{mM} 5.3$ in $0.1 \mathrm{M}$ $\left[\mathrm{NBu}_{4}\right]\left[\mathrm{PF}_{6}\right] \mathrm{CH}_{3} \mathrm{CN}$ solution. Scan rate $=100 \mathrm{mV} / \mathrm{s}$.

The diffusion coefficients for $\mathbf{5 . 2}$ and $\mathbf{5 . 3}$ were determined from the scan rate dependence of the $\mathrm{Co}^{\mathrm{II} / \mathrm{I}}$ peak current in the absence of acid.

$$
i_{c}=0.4463 n \mathcal{F} A C\left(\frac{n \mathcal{F} v D}{R T}\right)^{1 / 2} \quad \text { Equation } 5.1
$$

The Randles-Sevcik equation (Equation 5.1) was utilized to determine the diffusion coefficient, which relates the peak height, $i_{c}(\mathrm{~A})$, to analyte concentration $\mathrm{C}\left(\mathrm{mol} \mathrm{cm}^{-3}\right)$, area of the electrode surface A $\left(0.07 \mathrm{~cm}^{2}\right)$, scan rate $v\left(\mathrm{~V} \mathrm{~s}^{-1}\right)$, and diffusion coefficient of the analyte $\mathrm{D}\left(\mathrm{cm}^{2} \mathrm{~s}^{-1}\right) . F$ is Faraday's constant $\left(96485 \mathrm{C} \mathrm{mol}^{-1}\right), \mathrm{n}$ is the number of electrons appearing in a half reaction for the redox couple $(\mathrm{n}=1$ for $5.3, \mathrm{n}=2$ for 5.2 ), $\mathrm{R}$ is the universal gas constant $\left(8.314 \mathrm{~J} \mathrm{~mol}^{-1} \mathrm{~K}^{-1}\right)$, and $\mathrm{T}$ is temperature $(\mathrm{K})$. Plots of peak height current vs. (scan rate $)^{1 / 2}$ showed a linear relationship, indicating a diffusion controlled process, and the slope of the linear fit $\left(b=i_{c} / v^{1 / 2}\right)$ was utilized to determine diffusion coefficient (Figure 5.4, Figure 5.5). Each experiment was repeated three times, and calculated diffusion coefficients were consistent between runs. As expected, bulkier 5.2 has a substantially smaller value for the diffusion coefficient $\left(2.0 \times 10^{-6} \mathrm{~cm}^{2} \mathrm{~s}^{-1}\right)$ than the mononuclear complex $5.3\left(8.0 \times 10^{-6} \mathrm{~cm}^{2} \mathrm{~s}^{-1}\right)$. 

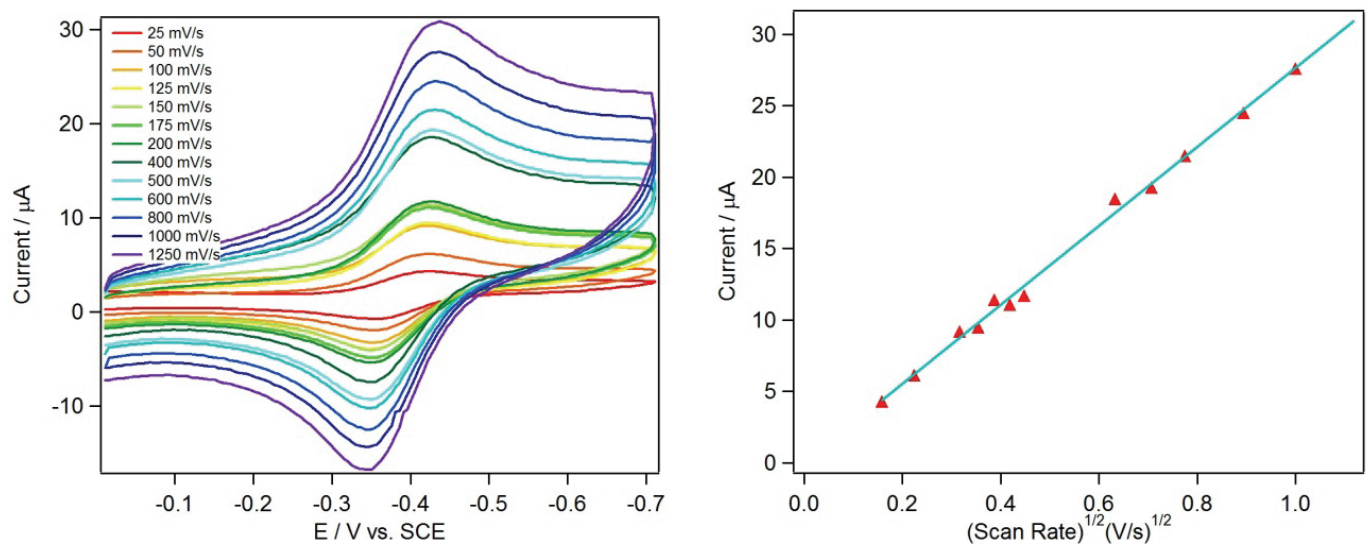

Figure 5.4 (left) Cyclic voltammograms of $0.372 \mathrm{mM} \mathrm{5.2} \mathrm{in} 0.1 \mathrm{M}$ $\left[\mathrm{NBu}_{4}\right]\left[\mathrm{PF}_{6}\right] \mathrm{CH}_{3} \mathrm{CN}$ solution at varying scan rates. (right) Peak height current vs. $(\text { scan rate })^{1 / 2}$ with linear fit. Slope $=2.76 \times 10^{-5}$ $\mu \mathrm{A} \mathrm{V}^{-1 / 2} \mathrm{~s}^{-1}$.
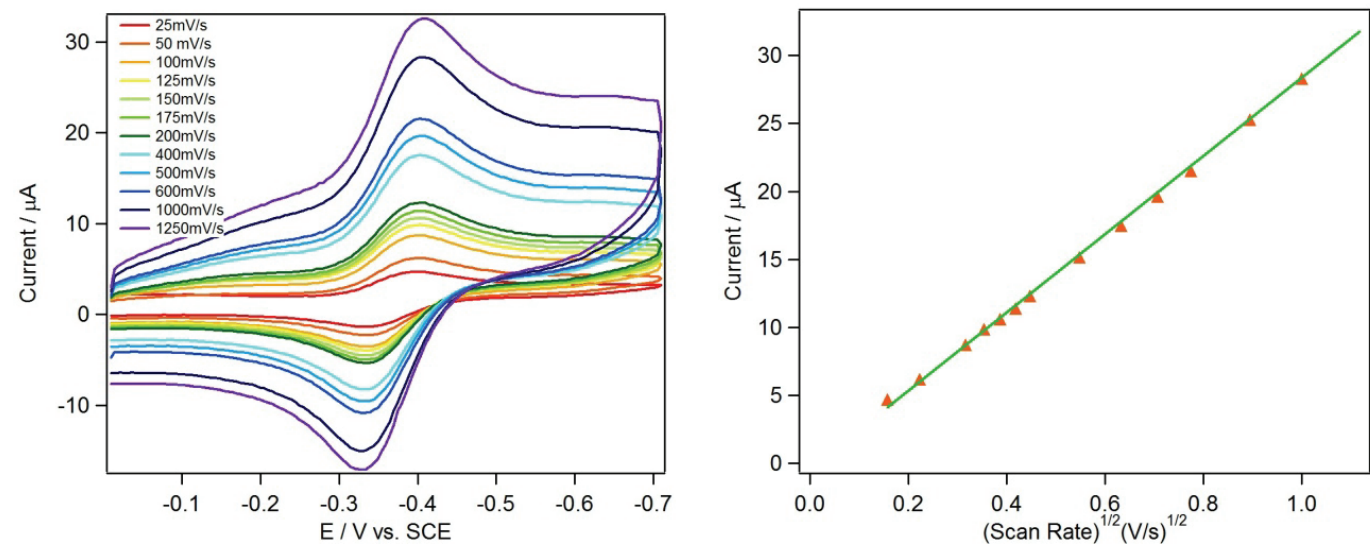

Figure 5.5 (left) Cyclic voltammograms of $0.649 \mathrm{mM} \mathrm{5.2} \mathrm{in} 0.1 \mathrm{M}$ $\left[\mathrm{NBu}_{4}\right]\left[\mathrm{PF}_{6}\right] \mathrm{CH}_{3} \mathrm{CN}$ solution at varying scan rates. (right) Peak height current vs. $(\text { scan rate })^{1 / 2}$ with linear fit. Slope $=2.93 \times 10^{-5}$ $\mu \mathrm{A} \mathrm{V}^{-1 / 2} \mathrm{~s}^{-1}$.

Upon addition of $\mathrm{TsOH} \cdot \mathrm{H}_{2} \mathrm{O}$ ( $p$-toluenesulfonic acid monohydrate) to solutions of both 5.2 and 5.3 in an inert atmosphere, catalytic waves were observed at a potential slightly negative of the formal reduction potential, $\mathrm{E}^{\circ}\left(\mathrm{Co}^{\mathrm{II} / \mathrm{I}}\right)$, of the species, indicating catalytic hydrogen evolution (Figure 5.6, Figure 5.7). Similar to the electrocatalytic behavior of 
$\left.\mathrm{Co}(\mathrm{dpgBF})_{2}\right)_{2}\left(\mathrm{CH}_{3} \mathrm{CN}\right)_{2}$ and $\mathrm{Co}\left(\mathrm{dmgBF}_{2}\right)_{2}\left(\mathrm{CH}_{3} \mathrm{CN}\right)_{2}$, catalytic waves at low acid concentrations exhibited peak-like shapes, indicative of an electrocatalytic process wherein catalysis occurs rapidly enough that current is controlled by diffusion of acid to the electrode surface.

Under higher acid-to-catalyst ratios, the catalytic waves approached a plateau shape. Under conditions where acid concentration is not depleted in the reaction region, a plateau shape of catalytic current indicates that the rate of reduction of the catalytic species at the electrode is equivalent to the rate of its reoxidation by the acid.
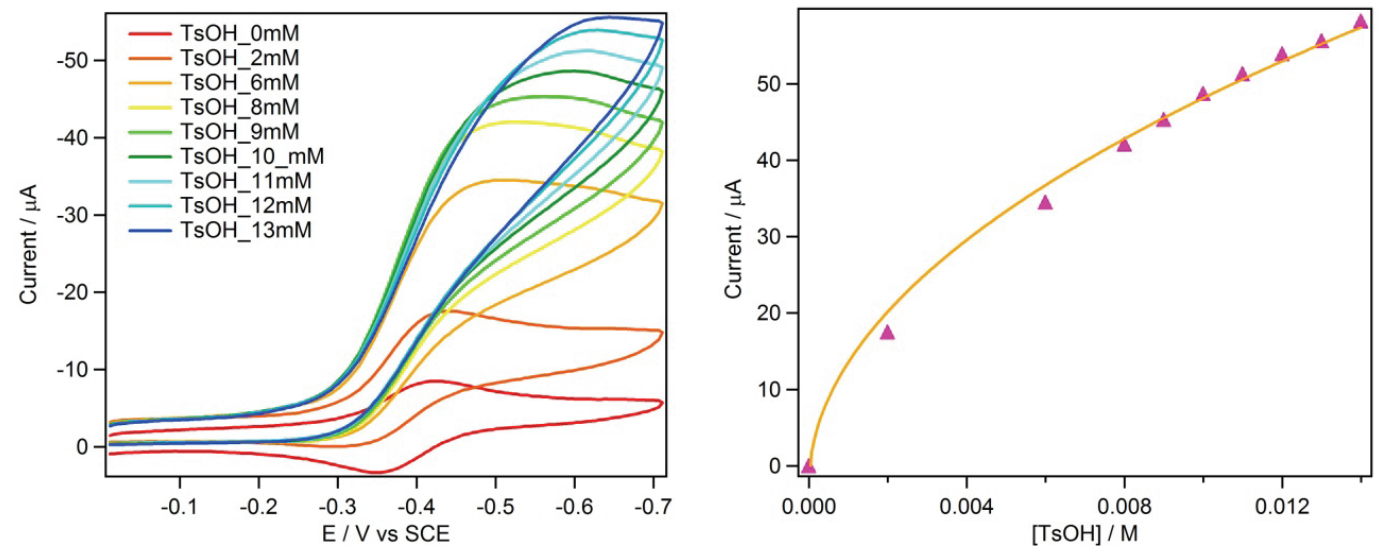

Figure 5.6 (left) Cyclic voltammogram of $0.372 \mathrm{mM} 5.2$ in $0.1 \mathrm{M}$ $\left[\mathrm{NBu}_{4}\right]\left[\mathrm{PF}_{6}\right]$ at a scan rate of $100 \mathrm{mV} \mathrm{s}^{-1}$ in the presence of $p$ toluene sulfonic acid monohydrate (right). Dependence of catalytic plateau current on acid concentration, fit to $i_{c}=$ $\mathrm{b}[\mathrm{TsOH}]^{1 / 2}+\mathrm{c}, \mathrm{b}=5.05 \times 10^{-4} \mathrm{~A} \mathrm{M}^{-1}, \mathrm{c}=-2.4 \times 10^{-6} \mathrm{~A}$. The plateau current was set to zero in the absence of acid, as predicted by Equation 5.2, even though the catalyst has a nonzero peak current under such conditions. 

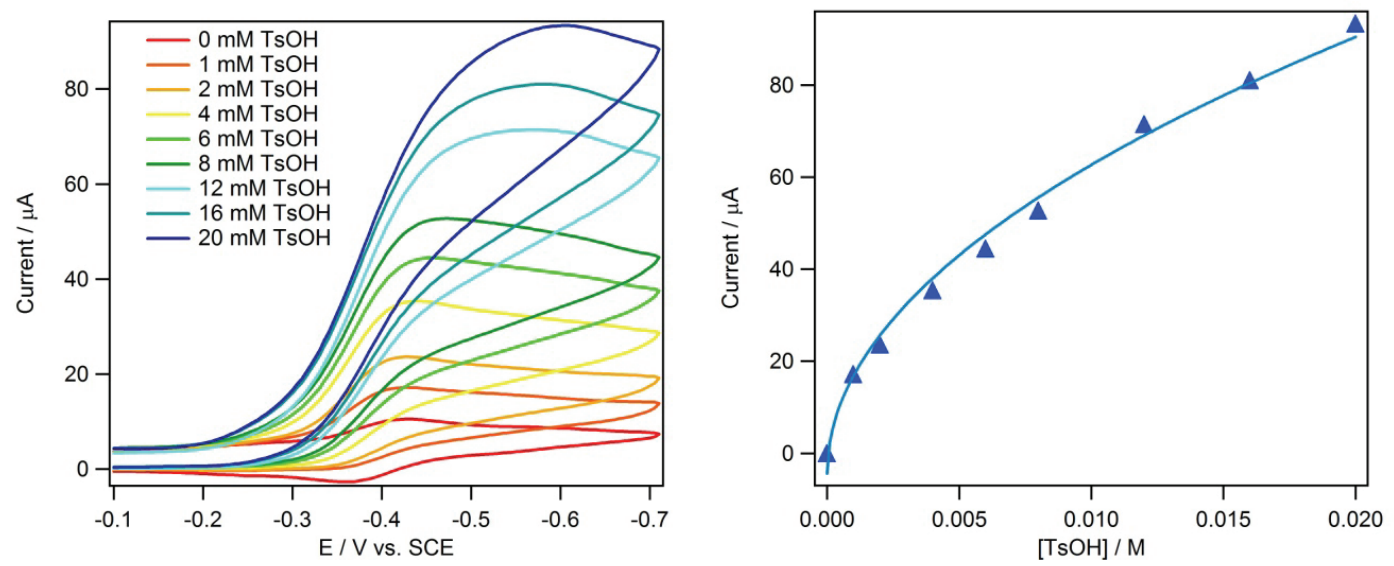

Figure 5.7 (left) Cyclic voltammogram of $0.524 \mathrm{mM} 5.3$ in $0.1 \mathrm{M}$ $\left[\mathrm{NBu}_{4}\right]\left[\mathrm{PF}_{6}\right]$ at a scan rate of $100 \mathrm{mV} \mathrm{s}^{-1}$ in the presence of $p$ toluene sulfonic acid monohydrate (right). Dependence of catalytic plateau current on acid concentration, fit to $i_{c}=$ $\mathrm{b}[\mathrm{TsOH}]^{1 / 2}+\mathrm{c}, \mathrm{b}=6.7 \times 10^{-4} \mathrm{~A} \mathrm{M}^{-1}, \mathrm{c}=-4.4 \times 10^{-6} \mathrm{~A}$. The plateau current was set to zero in the absence of acid, as predicted by Equation 5.2, even though the catalyst has a nonzero peak current under such conditions.

The plateau current of both $\mathbf{5 . 2}$ and $\mathbf{5 . 3}$ follow a first-order dependence on the concentration of acid and are less than first-order in concentration of catalyst, similar to the electrocatalytic behavior observed for $\mathrm{Co}\left(\mathrm{dmgBF}_{2}\right)_{2}\left(\mathrm{CH}_{3} \mathrm{CN}\right)_{2}$ (Figure 5.6, Figure 5.7, Figure 5.8, Figure 5.9). ${ }^{2}$ However, for calculation of the overall rate constant for $\mathrm{H}_{2}$ evolution, the reaction was taken as first-order in both acid and catalyst concentration (vide infra). 

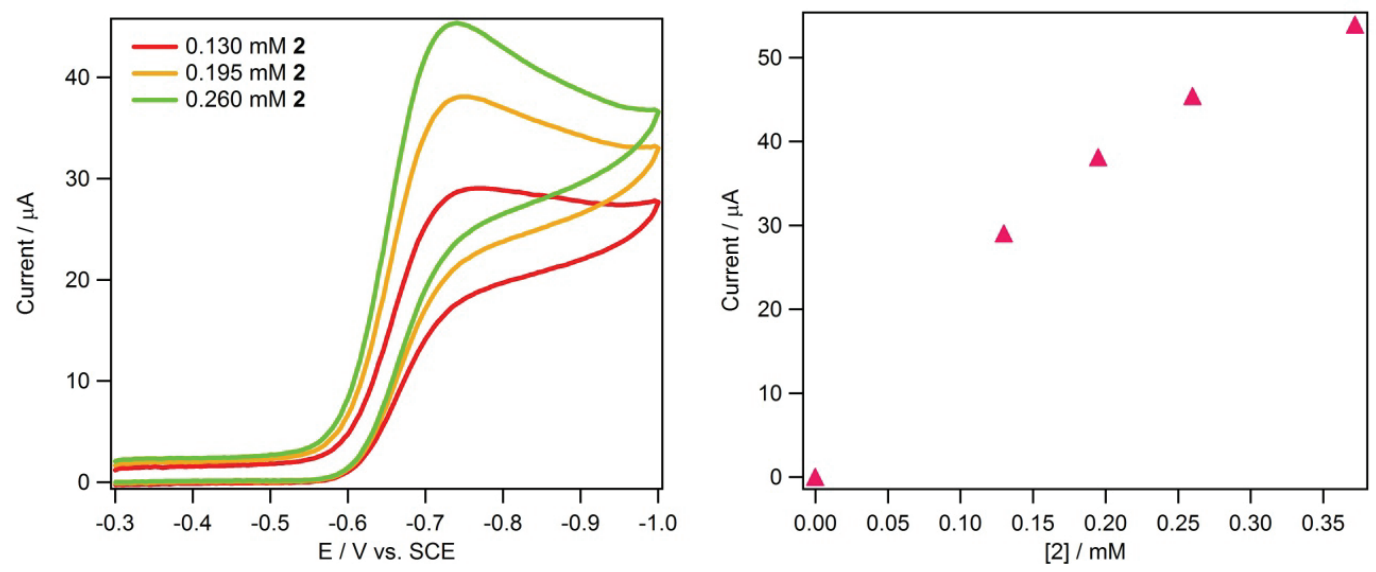

Figure 5.8 Dependence of catalytic plateau current on concentration of catalyst $\mathbf{5 . 2}$ at $12 \mathrm{mM}$ p-toluene sulfonic acid monohydrate in $0.1 \mathrm{M}\left[\mathrm{NBu}_{4}\right]\left[\mathrm{PF}_{6}\right]$ at a scan rate of $100 \mathrm{mV} \mathrm{s}{ }^{-1}$. The plateau current was set to zero in the absence of acid, as predicted by Equation 5.2, even though the catalyst has a nonzero peak current under such conditions.
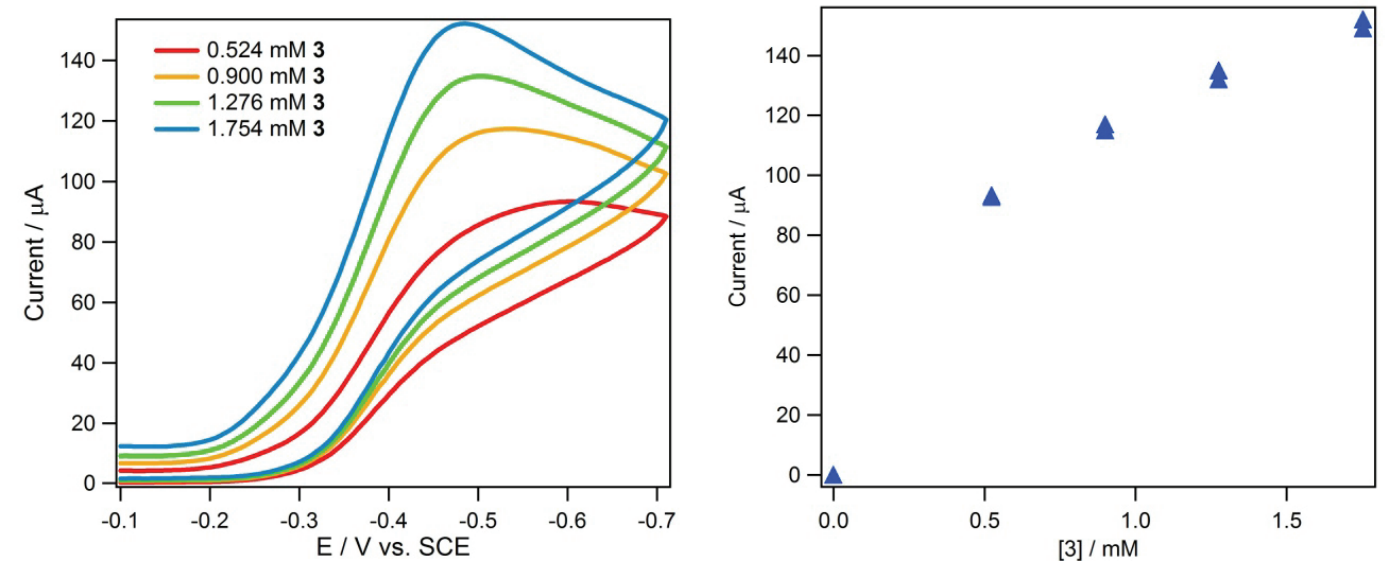

Figure 5.9 Dependence of catalytic plateau current on concentration of catalyst $\mathbf{5 . 3}$ at $20 \mathrm{mM}$ p-toluene sulfonic acid monohydrate in $0.1 \mathrm{M}\left[\mathrm{NBu}_{4}\right]\left[\mathrm{PF}_{6}\right]$ at a scan rate of $100 \mathrm{mV} \mathrm{s}^{-1}$. The plateau current was set to zero in the absence of acid, as predicted by Equation 5.2, even though the catalyst has a nonzero peak current under such conditions.

The overall rate constant for $\mathrm{H}_{2}$ evolution was estimated from the height of the catalytic plateau current for $\mathbf{5 . 2}$ and 5.3. For a reaction that is first order in both catalyst and acid, 
the current in the plateau region $\left(i_{c}\right.$, in $\left.\mathrm{A}\right)$ is related to the concentrations of catalyst ([Co], in $\left.\mathrm{mol} \mathrm{cm}{ }^{-3}\right)$ and acid $\left(\left[\mathrm{TsOH} \cdot \mathrm{H}_{2} \mathrm{O}\right]\right.$, in $\left.\mathrm{M}\right)$ in $\mathrm{M}$ by

$$
i_{c}=n \mathcal{F} A[C o](D k[T s O H])^{1 / 2} \text { Equation } 5.2
$$

where $n$ is the number of electrons for the overall $\mathrm{H}_{2}$ evolution reaction $(\mathrm{n}=2$ for $5.3, \mathrm{n}=4$ for 5.2), $F$ is Faraday's constant $\left(96,485 \mathrm{C} \mathrm{mol}^{-1}\right), A$ is the area of the electrode surface $(0.07$ $\left.\mathrm{cm}^{2}\right), D$ is the diffusion coefficient $\left(\mathrm{cm}^{2} \mathrm{~s}^{-1}\right)$, and $k$ is the reaction rate constant $\left(\mathrm{M}^{-1} \mathrm{~s}^{-1}\right)$. A plot of $i_{c}$ vs. [TsOH] was fit to the equation $i_{c}=\mathrm{bx}^{1 / 2}+\mathrm{c}$, where $\mathrm{b}=\mathrm{nFA}[\mathrm{Co}](\mathrm{D} k)^{1 / 2}$. The plateau current was set to zero in the absence of acid, as predicted by equation 5.2, even though the catalyst has a nonzero peak current under such conditions.

From the relationship of peak current vs. concentration of $\mathrm{TsOH}^{\cdot} \mathrm{H}_{2} \mathrm{O}$, estimates for the overall catalytic rate constants for hydrogen evolution were made (Figure 5.6, Figure 5.7). The apparent rate constant for $5.2,1200 \mathrm{M}^{-1} \mathrm{~s}^{-1}$, is similar to that for $5.3,800 \mathrm{M}^{-1} \mathrm{~s}^{-1}$, and both lie between the measured values for $\mathrm{Co}\left(\mathrm{dpgBF}_{2}\right)_{2}\left(\mathrm{CH}_{3} \mathrm{CN}\right)_{2}$ and $\mathrm{Co}\left(\mathrm{dmgBF}_{2}\right)_{2}\left(\mathrm{CH}_{3} \mathrm{CN}\right)_{2}$ (Table 5.1). Taken together, these results support the previous assignment of a reaction pathway in which the formation of the hydride is rate limiting: catalysis is first order in acid, less than first order in catalyst, and the binuclear systems exhibits no enhancement in activity. 
Table 5.1 Reduction potentials, apparent rate constants, and diffusion coefficients for cobaloxime catalysts.

\begin{tabular}{llllll}
\hline Complex & $\mathbf{E}^{\circ}(\mathrm{Co}(\mathrm{III} / \mathrm{II}))$ & $\mathbf{E}^{\text {o’ }}(\mathrm{Co}(\mathrm{II} / \mathrm{I}))$ & $\mathbf{k}_{\text {app }}\left(\mathbf{M}^{-1} \mathbf{s}^{-1}\right)$ & $\mathbf{D}\left(\mathbf{c m}^{2} / \mathbf{s}\right)$ & Ref \\
\hline $\mathbf{C o}\left(\mathbf{d m g B F}_{2}\right)_{2}\left(\mathrm{CH}_{3} \mathrm{CN}\right)_{2}$ & $\sim 0.2$ & -0.55 & 7000 & $8.0 \times 10^{-6 a}$ & 2 \\
$\mathbf{C o}\left(\mathbf{d p g B F}_{2}\right)_{2}\left(\mathrm{CH}_{3} \mathrm{CN}\right)_{2}$ & $\sim 0.3$ & -0.28 & 200 & $1.4 \times 10^{-6 a}$ & 2 \\
$\mathbf{5 . 2}$ & $\sim 0.2$ & -0.37 & 1200 & $2.0 \times 10^{-6 b}$ & This work \\
$\mathbf{5 . 3}$ & $\sim 0.2$ & -0.37 & 800 & $8.0 \times 10^{-6 b}$ & This work \\
\hline
\end{tabular}

${ }^{a}$ Diffusion coefficient determined by simulation of CV in absence of acid ${ }^{b}$ Diffusion coefficient determined from scan rate dependence.

\section{Conclusions}

In summary, we have prepared a novel binuclear cobaloxime $\mathbf{5 . 2}$ that is an efficient catalyst for $\mathrm{H}_{2}$ evolution and gleaned key mechanistic details of catalytic hydrogen evolution by directly comparing the catalytic rates to a mononuclear analogue 5.3. Electrochemical experiments reveal no enhancement in the rate of $\mathrm{H}_{2}$ evolution, further supporting the previous conclusions of Peters and coworkers, who showed electrocatalysis is first order in acid and first order or less than first order in catalyst. If a homolytic reaction pathway for hydrogen evolution predominates, covalent surface attachment of a mononuclear catalyst to an electrode will likely inhibit cooperative activity while a binuclear species such as $\mathbf{5 . 2}$ will maintain catalytic function. With this new catalyst in hand, we are poised to carry out those experiments.

\section{Acknowledgement}

This work was carried out in close collaboration with Carolyn N. Valdez. We thank Ian Stewart, Alex Miller, Bryan Stubbert, Charles McCrory, Xile Hu, and Jonas Peters for insightful discussions. This work was supported by the NSF Center for Chemical 
Innovation (Powering the Planet, CHE-0802907 and CHE-0947829), the Arnold and Mabel Beckman Foundation, CCSER (Gordon and Betty Moore Foundation), and the BP $\mathrm{MC}^{2}$ program. CNV is grateful for support from the Caltech Summer Undergraduate Research Program

\section{Experimental Details}

\section{Reagents}

All synthetic procedures and electrochemical measurements were performed under anaerobic and anhydrous conditions under an atmosphere of argon or nitrogen, with the exception of the preparation of the ligand. Dry acetonitrile was prepared by passing commercially available dry solvent over an activated alumina column and was stored on $4 \AA$ sieves under nitrogen. Tetrabutylammonium hexafluorophosphate was recrystallized from ethanol before use. All other reagents were purchased from chemical suppliers and used as received. $\mathrm{ClCo}(\mathrm{dmgH})(\mathrm{dpgH})$ py $\left(\mathrm{dmgH}_{2}=\right.$ dimethylglyoxime, $\mathrm{dpgH}_{2}=$ diphenylglyoxime, py $=$ pyridine) was prepared by the method of Gupta et al. ${ }^{14}$

\section{Physical Methods}

${ }^{1} \mathrm{H}$ and ${ }^{19} \mathrm{~F}$ NMR measurements were recorded on a Varian MERCURY $300 \mathrm{MHz}$ spectrometer and analyzed using MestReNova (version 6.1.1). ${ }^{1} \mathrm{H}$ NMR spectra were referenced to internal solvents and ${ }^{19} \mathrm{~F}$ spectra were referenced using an external $\mathrm{C}_{6} \mathrm{~F}_{6}$ standard.

Electrospray mass spectrometry measurements were recorded on a Finnigan LCQ ion trap mass spectrometer. High resolution mass spectrometry (FAB) measurements were recorded using a JEOL JMS-600H High Resolution Mass Spectrometer. 
Electrochemical measurements were made with a Pine Instruments WaveNow potentiostat and the data were processed using Igor Pro 5.01 (Wavemetrics). Electrochemical analysis was carried out in a three-electrode cell, consisting of a glassy carbon working electrode (surface area $=0.07 \mathrm{~cm}^{2}$ ), a platinum wire counter electrode, and a freshly prepared $\mathrm{Ag} / \mathrm{AgNO}_{3}(0.01 \mathrm{M})$ reference electrode filled with $0.1 \mathrm{M}\left[{ }^{n} \mathrm{Bu}_{4}\right]\left[\mathrm{PF}_{6}\right]$ acetonitrile solution. All potentials were referenced to $\mathrm{Fc} / \mathrm{Fc}^{+}$as an internal standard and converted to $\mathrm{SCE}\left(\mathrm{E}^{\circ}\left(\mathrm{Fc} / \mathrm{Fc}^{+}\right)=0.380 \mathrm{~V}\right.$ vs. $\left.\mathrm{SCE}\right)$.

\section{Synthetic Details}

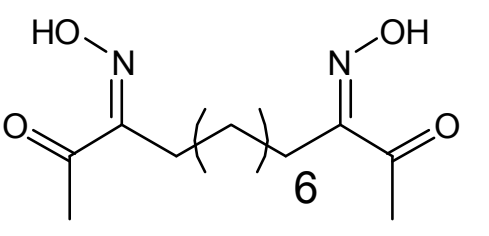

Tetradecane-2,3,12,13-tetraone 3,12-dioxime (5.4a) Under an argon atmosphere, sodium metal (1.5 g, $65 \mathrm{mmol})$ was dissolved in $100 \mathrm{~mL}$ of absolute ethanol. Ethyl acetoacetate

$(8.45 \mathrm{~g}, 65 \mathrm{mmol})$ was syringed into the stirring solution and allowed to react. After 10 minutes, the yellow solution was heated to reflux, followed by the addition of 1,8 dibromooctane $(8.02 \mathrm{~g}, 29.5 \mathrm{mmol})$ and exposure to atmosphere. The solution was allowed to react for $12 \mathrm{~h}$ until neutral, followed by the removal of solvent via rotary evaporation to yield a viscous oil containing a white precipitate $(\mathrm{NaBr})$. Enough water was added to the product to dissolve the precipitate, and the mixture was extracted with ether. The ether layers were combined and dried over anhydrous magnesium sulfate, and the ether was removed via rotary evaporation. To the resulting clear oil an aqueous solution of potassium hydroxide (4.51 g, $80 \mathrm{mmol}$ ) was added and hydrolysis was effected for $3 \mathrm{~h}$ at room temperature, producing a cloudy, bright yellow solution. The mixture was cooled with an ice bath followed by the addition of sodium nitrite $(4.26 \mathrm{~g}, 62 \mathrm{mmol})$ and then $10 \%$ sulfuric acid was added dropwise, until an aliquot of the yellow solution turned the color of a potassium iodide/starch test dark blue for 15 minutes. Aqueous potassium hydroxide was 
added until the mixture became homogeneous, followed by extraction with ether to remove residual starting materials. The yellow mixture was cooled and $4 \mathrm{M}$ sulfuric acid was added until the solution was acidified $(\mathrm{pH} \approx 5)$. The resulting solid was extracted with ether, washed with water and a saturated sodium carbonate solution, and dried over $\mathrm{MgSO}_{4}$. Ether was removed via rotary evaporation to yield a cream solid (2.4 g, 28\%). ${ }^{1} \mathrm{H}$ NMR (300 MHz, $\left.\left(\mathrm{CD}_{3}\right)_{2} \mathrm{CO}\right): \delta 10.4$ (s, $2 \mathrm{H}$, oxime), $\delta 2.48$ (t, $\left.4 \mathrm{H}, \mathrm{J}=7.3 \mathrm{~Hz}, \alpha-\mathrm{CH}_{2}\right), \delta 2.26$ (s, $\left.6 \mathrm{H}, \mathrm{CH}_{3}\right), \delta 1.5-1.25\left(\mathrm{~m}, 12 \mathrm{H}, \mathrm{CH}_{2}\right) \mathrm{ppm}$.<smiles>C/C(=N\O)C(C)CCC(C)/C(C)=N/O</smiles>

Tetradecane-2,3,12,13-tetraone tetraoxime $(5.4$, $\left.\mathbf{L H}_{4}\right) \quad 5.4 \mathrm{a}(2.4 \mathrm{~g}, \quad 8.4 \mathrm{mmol})$, hydroxylamine hydrochloride $(2.3 \mathrm{~g}, 33.5 \mathrm{mmol})$, and sodium

carbonate $(2.45 \mathrm{~g}, 17.7 \mathrm{mmol})$ were suspended in ethanol $(100 \mathrm{~mL})$. Water was added slowly (20 mL) until all solids dissolved and the solution was refluxed for $14 \mathrm{~h}$. The white precipitate that formed was collected, washed with water until chloride was no longer detected in the filtrate, and dried (1.65g, 63\%). ${ }^{1} \mathrm{H} \mathrm{NMR}\left(300 \mathrm{MHz},\left(\mathrm{CD}_{3}\right)_{2} \mathrm{CO}\right): \delta 10.46(\mathrm{~s}, 2$ $\mathrm{H}$, oxime $\mathrm{a}_{\mathrm{a}}$, $\delta 10.41(\mathrm{~s}, 2 \mathrm{H}$, oxime $\mathrm{b}), \delta 2.60\left(\mathrm{t}, 4 \mathrm{H}, \mathrm{J}=7.5 \mathrm{~Hz}, \mathrm{a}-\mathrm{CH}_{2}\right), \delta 1.96\left(\mathrm{~s}, 6 \mathrm{H}, \mathrm{CH}_{3}\right), \delta$ 1.41-1.30 (m, $12 \mathrm{H}, \mathrm{CH}_{2}$ ) ppm. Electrospray MS (50:50 MeOH: $\left.\mathrm{H}_{2} \mathrm{O}\right), m / z$ calculated for $\mathrm{C}_{14} \mathrm{H}_{26} \mathrm{~N}_{4} \mathrm{O}_{4}:$ 314.2. Found: $315.0(\mathrm{M}), 296.9(\mathrm{M}-\mathrm{OH})$.

\section{$\mathrm{ClCo}(\mathrm{dpgH})(\mathrm{py})-\mathrm{LH}_{2}-\mathrm{ClCo}(\mathrm{dpgH}) \mathrm{py} ; \quad \mathrm{LH}_{4}=\quad$ tetradecane-2,3,12,13-tetraone} tetraoxime, $\mathrm{py}=$ pyridine, $\mathbf{d p g H}_{2}=$ diphenylglyoxime) (5.1) Under an argon atmosphere, anhydrous pyridine $(0.79 \mathrm{~g}, 10 \mathrm{mmol})$ was added by syringe to a refluxing solution of cobalt(II) chloride (0.45 g, $1.9 \mathrm{mmol})$, diphenylglyoxime (0.49 g, $2.0 \mathrm{mmol})$, and $5.4(0.3 \mathrm{~g}$, $0.9 \mathrm{mmol})$ in degassed ethanol $(50 \mathrm{~mL})$. The heterogeneous mixture was cooled to room temperature and air was bubbled through the solution for at least $6 \mathrm{~h}$. The solvent was removed via rotary evaporation to yield a brown solid, which was dissolved in ethyl acetate 
and filtered through a silica gel plug to separate an insoluble green material. Ethyl acetate was evaporated, and the brown material was dissolved in a minimal amount of chloroform and loaded onto a silica gel column pre-eluted with chloroform. Three bands were observed with 1:9 ethyl acetate:chloroform. The first band, corresponding to the monomeric species $\mathrm{ClCo}(\mathrm{dpgH})_{2} \mathrm{py}$, eluted with 1:9 ethyl acetate:chloroform, and the second and third bands eluted together with 1:1 ethyl acetate:chloroform. The second band corresponds to the half metallated ligand, $\mathrm{ClCo}(\mathrm{dpgH})\left(\mathrm{LH}_{3}\right)$ py and the third to the desired product. Solvent was evaporated from this mixture, then the resulting solid was dissolved in minimal chloroform and loaded onto a longer, thinner column. The two products were separated with 15:85 ethyl acetate:chloroform, to yield the desired product as a brown crystalline solid (0.11 g, 10\%). ${ }^{1} \mathrm{H}$ NMR $\left(300 \mathrm{MHz}, \mathrm{CD}_{3} \mathrm{CN}\right): \delta 18.5$ (s, $\left.4 \mathrm{H},-\mathrm{OHO}-\right), \delta 8.29$ $(\mathrm{m}, 4 \mathrm{H}$, py $\mathrm{CH}(2,6)), \delta 7.86(\mathrm{~m}, 4 \mathrm{H}$, py $\mathrm{CH}(3,5)), \delta 7.39(\mathrm{~m}, 2 \mathrm{H}$, py $\mathrm{CH}(4)), \delta 7.4-7.1(\mathrm{~m}$, $20 \mathrm{H}, \mathrm{Ph}), \delta 2.85\left(\mathrm{~m}, 4 \mathrm{H}, \mathrm{a}-\mathrm{CH}_{2}\right), \delta 2.39\left(\mathrm{~s}, 6 \mathrm{H}, \mathrm{CH}_{3}\right), \delta 1.55-1.05\left(\mathrm{~m}, 12 \mathrm{H}, \mathrm{CH}_{2}\right)$ ppm. HRMS (FAB+), $m / z$ calculated for $\mathrm{C}_{52} \mathrm{H}_{56} \mathrm{Cl}_{2} \mathrm{Co}_{2} \mathrm{~N}_{10} \mathrm{O}_{8}$ : 1136.23. Found: $1137.24(\mathrm{M}+\mathrm{H})$, 1022.3 (M - Cl - pyridine), $979.3\left(\mathrm{M}-\mathrm{C}_{6} \mathrm{H}_{5}\right.$ - pyridine - $\left.2 \mathrm{H}\right) 907.3(\mathrm{M}-2 \mathrm{Cl}-2$ pyridine $\left.+\mathrm{H}, \mathrm{M}-3 \mathrm{C}_{6} \mathrm{H}_{5}-\mathrm{H}\right), 783.2\left(\mathrm{M}-4 \mathrm{C}_{6} \mathrm{H}_{5}-\mathrm{Cl}-\mathrm{CH}_{3}\right), 704.2\left(\mathrm{M}-4 \mathrm{C}_{6} \mathrm{H}_{5}-\mathrm{CH}_{3}-\mathrm{Cl}-\right.$ pyridine), $668.2\left(\mathrm{M}-4 \mathrm{C}_{6} \mathrm{H}_{5}-\mathrm{CH}_{3}-2 \mathrm{Cl}\right.$ - pyridine $\left.-\mathrm{H}\right)$.

\section{$\left.\mathrm{ClCo}(\operatorname{dpgBF})_{2}\right)\left(\mathrm{CH}_{3} \mathrm{CN}\right)-\mathrm{L}\left(\mathrm{BF}_{2}\right)_{2}-\mathrm{ClCo}\left(\mathrm{dpgBF}_{2}\right)\left(\mathrm{CH}_{3} \mathrm{CN}\right) ; \quad \mathrm{LH}_{4}=$ tetradecane-} 2,3,12,13-tetraone tetraoxime, $\mathbf{d p g H}_{2}=$ diphenylglyoxime) (5.2) Under an atmosphere of argon, boron trifluoride diethyl etherate $(0.153 \mathrm{~g}, 1.1 \mathrm{mmol})$ was syringed into a brown, refluxing solution of $5.1(0.15 \mathrm{~g}, 0.13 \mathrm{mmol})$ in dry acetonitrile $(25 \mathrm{~mL})$. The solution turned red-brown and was allowed to reflux for $1 \mathrm{~h}$, then the solvent was evaporated to yield a red-brown solid, which was recrystallized from acetonitrile and diethyl ether $(0.15 \mathrm{~g}$, 91\%). ${ }^{1} \mathrm{H}$ NMR (300 MHz, $\left.\mathrm{CD}_{3} \mathrm{Cl}\right): \delta 7.4-7.2(\mathrm{~m}, 20 \mathrm{H}, \mathrm{Ph}), \delta 3.08$ (m, $\left.4 \mathrm{H}, \alpha-\mathrm{CH}_{2}\right), \delta 2.67$ (s, 6H, $\left.\mathrm{CH}_{3}\right), \delta 1.7-1.3\left(\mathrm{~m}, 12 \mathrm{H}, \mathrm{CH}_{2}\right)$ ppm. ${ }^{19} \mathrm{~F}$ NMR $\left(300 \mathrm{MHz}, \mathrm{CD}_{3} \mathrm{CN}\right): \delta-151$ (s) ppm. 
$\mathrm{ClCo}\left(\mathrm{dmgBF}_{2}\right)\left(\mathrm{dpgBF}_{2}\right)\left(\mathrm{CH}_{3} \mathrm{CN}\right) \quad\left(\mathrm{dmg}=\right.$ dimethylglyoxime, $\operatorname{dpg}=^{125}$ diphenylglyoxime) (5.3) Under an atmosphere of argon, $\mathrm{ClCo}(\mathrm{dmgH})(\mathrm{dpgH}) \mathrm{py}(0.24 \mathrm{~g}$, $0.455 \mathrm{mmol})$ was dissolved in dry acetonitrile $(15 \mathrm{~mL})$. Boron trifluoride diethyl etherate ( $0.646 \mathrm{~g}, 4.55 \mathrm{mmol}$ ) was syringed into the brown solution. The resulting red solution was stirred and heated to reflux for $1 \mathrm{~h}$, then allowed to stir at room temperature for $3 \mathrm{~h}$. The solvent was evaporated, and the resulting red oily solid was redissolved in acetonitrile (4 $\mathrm{mL})$. Diethyl ether $(15 \mathrm{~mL})$ was added until a white solid crashed out. The solution was filtered, and the solvent was removed from the red-brown solution. The oily solid was dissolved in ethyl acetate $(8 \mathrm{~mL})$ and washed with water $(2 \mathrm{~mL})$, then dried over $\mathrm{MgSO}_{4}$. The solvent was evaporated to yield a red-brown solid (0.079 g, 30\%). ${ }^{1} \mathrm{H}$ NMR $(300 \mathrm{MHz}$, $\left.\mathrm{CD}_{3} \mathrm{CN}\right): \delta 7.5-7.3(\mathrm{~m}, 10 \mathrm{H}, \mathrm{Ph}), \delta 2.68\left(\mathrm{~s}, 6 \mathrm{H}, \mathrm{CH}_{3}\right) \mathrm{ppm} .{ }^{19} \mathrm{~F} \mathrm{NMR}\left(300 \mathrm{MHz}, \mathrm{CD}_{3} \mathrm{CN}\right): \delta$ -151 (s) ppm. HRMS (FAB+), $m / z$ calculated for $\mathrm{C}_{20} \mathrm{H}_{19} \mathrm{BClCoF}_{4} \mathrm{~N}_{5} \mathrm{O}_{4}: 585.06$ Found: $585.06(\mathrm{M}), 566.05(\mathrm{M}-\mathrm{F}), 509.03\left(\mathrm{M}-\mathrm{C}_{6} \mathrm{H}_{5}\right), 490.03\left(\mathrm{M}-\mathrm{F}-\mathrm{C}_{6} \mathrm{H}_{5}\right), 461.04\left(\mathrm{M}-\mathrm{C}_{6} \mathrm{H}_{5}-\right.$ $\left.\mathrm{BF}_{2}+\mathrm{H}\right), 442.01\left(\mathrm{M}-\mathrm{C}_{6} \mathrm{H}_{5}-\mathrm{BF}_{2}-\mathrm{F}+\mathrm{H}\right)$.

\section{References}

1. Dempsey, J. L.; Brunschwig, B. S.; Winkler, J. R.; Gray, H. B. Acc. Chem. Res. 2009, 42, 1995.

2. Hu, X.; Brunschwig, B. S.; Peters, J. C. J. Am. Chem. Soc. 2007, 129, 8988.

3. Dempsey, J. L.; Winkler, J. R.; Gray, H. B. J. Am. Chem. Soc. 2010, 132, 1060.

4. Dempsey, J. L.; Winkler, J. R.; Gray, H. B. J. Am. Chem. Soc. 2010.

5. Gray, H. B. Nat. Chem. 2009, 1, 7.

6. Berben, L. A.; Peters, J. C. Chem. Commun. 2010, 46, 398.

7. Le Goff, A.; Artero, V.; Jousselme, B.; Tran, P. D.; Guillet, N.; Metaye, R.; Fihri, A.; Palacin, S.; Fontecave, M. Science 2009, 326, 1384. 
8. Collman, J. P.; Ha, Y.; Wagenknecht, P. S.; Lopez, M. A.; Guilard, R. J. Am. Chem. Soc. 1993, 115, 9080.

9. Collman, J. P.; Wagenknecht, P. S.; Lewis, N. S. J. Am. Chem. Soc. 1992, 114, 5665.

10. Jacobsen, E. N. Acc. Chem. Res. 2000, 33, 421.

11. Venkatasubbaiah, K.; Gill, C. S.; Takatani, T.; Sherrill, C. D.; Jones, C. W. Chem. Eur. J. $2009,15,3951$.

12. Gill, C. S.; Venkatasubbaiah, K.; Phan, N. T. S.; Weck, M.; Jones, C. W. Chem-Eur J 2008, 14, 7306 .

13. Lance, K. A.; Goldsby, K. A.; Busch, D. H. Inorg. Chem. 1990, 29, 4537.

14. Gupta, B. D.; Yamuna, R.; Singh, V.; Tiwari, U.; Barclay, T.; Cordes, W. J. Organomet. Chem. 2001, 627, 80 .

15. Shimakoshi, H.; Koga, M.; Hisaeda, Y. Bull. Chem. Soc. Jpn. 2002, 75, 1553. 
Chapter 6

\section{BIFUNCTIONAL COBALOXIMES FOR COVALENT} ATTACHMENT TO SILICON ELECTRODES 


\section{Introduction}

A scalable solar-driven water splitting device will likely require catalysts to be interfaced directly with a photoelectrode surface. In a notable recent study, Fontecave and coworkers covalently grafted a derivative of the $\left[\mathrm{Ni}\left(\mathrm{P}^{\mathrm{Ph}}{ }_{2} \mathrm{~N}^{\mathrm{Ph}}\right)_{2}\right]^{2+}$ catalyst onto multiwalled carbon nanotubes to create a high surface area cathode material with high catalytic activity. ${ }^{1} \mathrm{~A}$ similar electrode with covalently linked cobaloximes is clearly desired, though to date has not been reported.

Efforts have been made, however, to characterize the electrochemistry of these catalysts bound to surfaces via noncovalent interactions. Early reports discussing electrodes chemically modified with the cobaloximes of interest have indicated that these catalysts do remain active on an electrode surface. Pantani and coworkers prepared cobaloxime modified electrodes by spin-coating a mixture of $\mathrm{Co}\left(\mathrm{dmgBF}_{2}\right)_{2}\left(\mathrm{CH}_{3} \mathrm{CN}\right)_{2}(\mathbf{1})$ and black carbon in a Nafion ${ }^{\oplus}$ film. $^{2}$ Electrocatalytic behavior was observed in preliminary experiments with these modified electrodes, but mass transport limitations across the Nafion ${ }^{\oplus}$ film hindered efficiency. In related work, Berben and Peters directly adsorbed $\mathrm{Co}\left(\mathrm{dmgBF}_{2}\right)_{2}\left(\mathrm{CH}_{3} \mathrm{CN}\right)_{2}$ onto a glassy carbon electrode to prepare their own chemically modified electrodes. These electrodes were active for $\mathrm{H}_{2}$ evolution in aqueous solution ( $\mathrm{pH}$ $<4.5)$ with a $100 \mathrm{mV}$ overpotential, ${ }^{3}$ and electrochemical analysis indicated that hydrogen evolution efficiency was controlled by kinetic limitations associated with the catalytic reduction of protons, not mass transport or electron transfer steps.

In order to prepare a controlled electrode surface, Berben and Peters also prepared a semicyclic cobaloxime related complex supported by $\mathrm{N}$-aryl Shiff base/oxime ligands $\left[\mathrm{Co}\left((\mathrm{DOR})_{2} \mathrm{BF}_{2}\right) \mathrm{Br}_{2}\right]$ containing a carboxylic acid substituent. ${ }^{3}$ The catalyst was adsorbed onto ITO electrodes to yield a chemically modified electrode with a surface coverage that 
corresponded to about a monolayer of catalyst. Unfortunately, over the timecourse of several hours, substantial leeching of the catalyst from the electrode occurred in acidic solution, as the carboxylate-ITO interaction is likely weakened under these conditions. Nevertheless, this work demonstrated efficient electrocommunication across the ITOcarboxylate interface and suggests a more robust covalent attachment of a catalyst to an electrode will aid in device development.

Silicon is a promising photocathode material for this proposed water splitting device. ${ }^{4}$ Upon exposure to air, however, oxide layers are readily formed on the electrode surfaces, which have deleterious effects on their photoelectrochemical properties. Alkylated silicon surfaces, however, exhibit enhanced stability towards oxidation and have a low density of surface trap states. ${ }^{5}$ Further, these modified surfaces provide access to many functional groups, opening the door for a rich array of secondary organometallic reactions that can be carried out on the electrode surface.

Alkylated surfaces are readily prepared via a two-step halogenation/alkylation process. ${ }^{6}$ Secondary Heck coupling and olefin cross-metathesis reactions have been carried out successfully on surfaces containing an allyl monolayer to link small molecules and polymers. ${ }^{7-8}$ However, steric issues limit the extent of functionalization accessible. Recently, though, the characterization of silicon surfaces passivated with mixed methyl/allyl and methyl/thienyl monolayers has been reported, and secondary reactions at the surface of these materials should be give higher yields as crowding issues will be mitigated. ${ }^{9-10}$

Based on the success of both Heck coupling and olefin cross-metathesis reactions at the surface of these passivated silicon electrodes, new cobaloxime catalysts containing an olefin group in the ligand backbone are immediately desired. Reaction of these functionalized molecules with $\operatorname{Si}(111)$ surfaces passivated with a methyl/allyl or a methyl/thienyl 
monolayer should yield a covalent electrode-cobaloxime linkage robust to catalytic conditions. Herein is described a new cobaloxime catalyst with functionality amenable for surface chemistry, homogeneous electrochemical studies of this complex, and initial efforts to prepare the corresponding chemically modified silicon electrodes.

\section{Results and Discussion}

\section{Synthesis}

Few reports on chemical derivatives of the glyoxime ligand are available, and none discuss glyoxime modification with a secondary functionality of relevance to these surface modification studies. In light of the mild conditions and ease of preparing the ligands for the binuclear cobaloximes discussed in Chapter 5 , a similar route for ligand preparation was sought. ${ }^{11}$

Ethyl acetoacetate was deprotonated with sodium ethoxide, prepared in situ from the reaction of sodium with absolute ethanol (Scheme 6.1). The solution was brought to reflux and 5-bromo-1-pentene was added and allowed to react, producing a $\beta$-ketoester. Saponification with potassium hydroxide followed by nitrosation with sodium nitrite yielded the a-oxime. Reaction with hydroxylamine hydrochloride produced with the desired ligand.

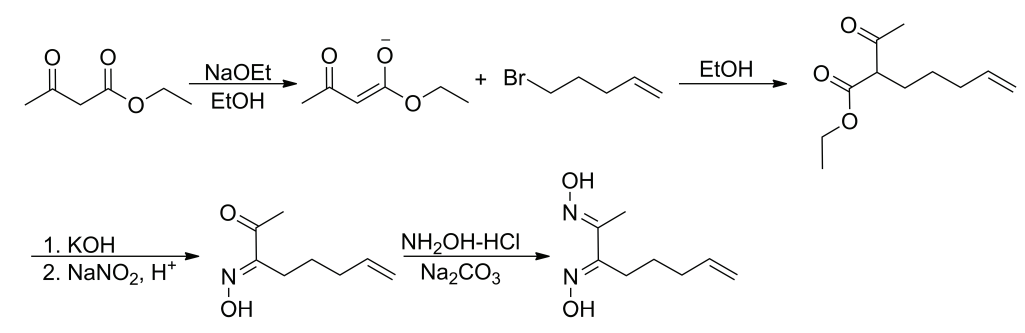


The functionalized glyoxime ligand, pentenyl-methylglyoxime (pmg), was utilized to prepare a cobaloxime. As polymerization of the terminal olefin is readily catalyzed by boron-trifluoride, a hydrogen-bridged cobalt(III) species was targeted. The preparation was adapted from Schrauzer's method for preparing chloro(pyridine)cobaloxime(III); ${ }^{12} 2$ equivalents of pentenyl-methylglyoxime were reacted with cobalt(II) chloride hexahydrate in degassed ethanol and the solution was brought to reflux. Two equivalents of pyridine were added, at which point the reaction mixture was brought to room temperature and air was bubbled through the solution for six hours to produce the desired cobaloxime, $\mathrm{ClCo}^{\mathrm{III}}(\mathrm{pmgH})_{2} \mathrm{py}($ Scheme 6.2).

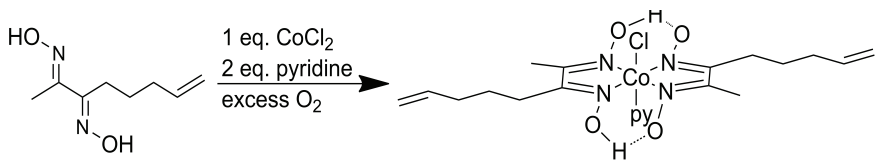

Scheme 6.2

Electrochemistry

Electrochemical measurements of $\mathrm{ClCo}^{\mathrm{III}}(\mathrm{pmgH})_{2}$ py were performed in $0.1 \mathrm{M}\left[\mathrm{NBu}_{4}\right]\left[\mathrm{PF}_{6}\right]$ acetonitrile solutions at a glassy carbon electrode. The cyclic voltammogram exhibits an irreversible $\mathrm{Co}^{\mathrm{III} / \mathrm{II}}$ reduction peak at $-0.38 \mathrm{~V}$ vs. SCE and a reversible $\mathrm{Co}^{\mathrm{II} / \mathrm{I}}$ couple at $-1.1 \mathrm{~V}$ vs. SCE (Figure 6.1). The corresponding processes for the dimethylglyoxime analogue, $\mathrm{ClCo}^{\mathrm{III}}(\mathrm{dmg})_{2} \mathrm{py}(\mathbf{3 a})$, occur at -0.68 and $-1.13 \mathrm{~V}$ vs. SCE, as measured by Eisenberg and coworkers. ${ }^{13}$ Upon addition of a weak acid, $\left[\mathrm{Et}_{3} \mathrm{NH}\right][\mathrm{OTs}]\left(\mathrm{pK}_{\mathrm{a}}=18.7\right)$, a catalytic wave was observed near the $\mathrm{Co}^{\mathrm{II} / \mathrm{I}}$ couple, corresponding to approximately a $230 \mathrm{mV}$ overpotential. ${ }^{14}$ At low acid concentrations, the catalytic peak onset occurred slightly positive of the $\mathrm{Co}^{\mathrm{II} / \mathrm{I}}$ couple, with the reduction peak appearing as a shoulder (Figure 6.2). As the acid concentration is increased, the catalytic current increases and the peak shifts to more 
negative potentials (Figure 6.3). A separate electrochemical feature for the $\mathrm{Co}^{\mathrm{II} / \mathrm{I}}$ potential was not observed under these conditions. Nearly identical observations were made by Fontecave and coworkers for $\mathrm{ClCo}^{\mathrm{III}}(\mathrm{dmg})_{2}$ py in DMF solutions. ${ }^{15}$ The peak-like shape of the catalytic waves suggests that catalytic hydrogen evolution proceeds sufficiently rapidly and the current is controlled, in part, by diffusion of acid to the electrode surface. ${ }^{16}$

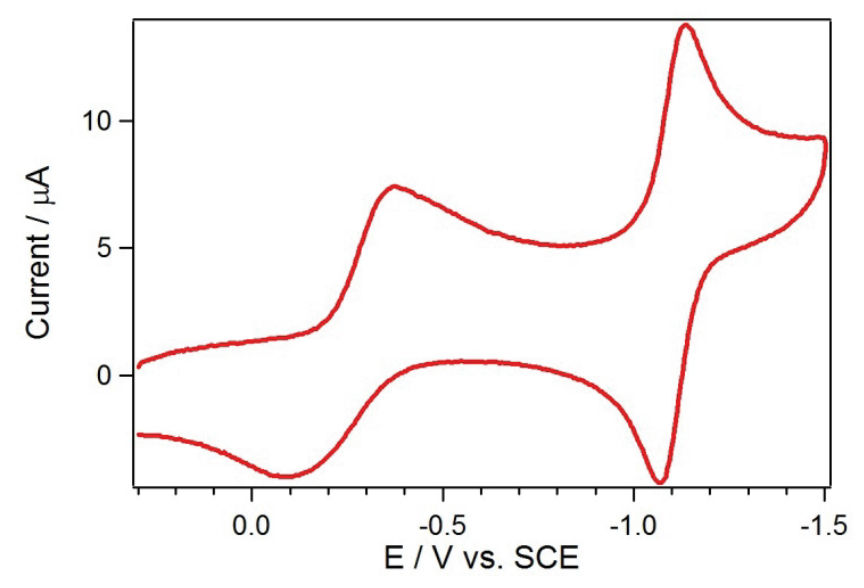

Figure 6.1 Cyclic voltammogram of $0.61 \mathrm{mM} \mathrm{ClCo}{ }^{\mathrm{III}}(\mathrm{pmgH})_{2} \mathrm{py}$ in $0.1 \mathrm{M}\left[\mathrm{NBu}_{4}\right]\left[\mathrm{PF}_{6}\right] \mathrm{CH}_{3} \mathrm{CN}$ solution. Scan rate $=100 \mathrm{mV} / \mathrm{s}$.

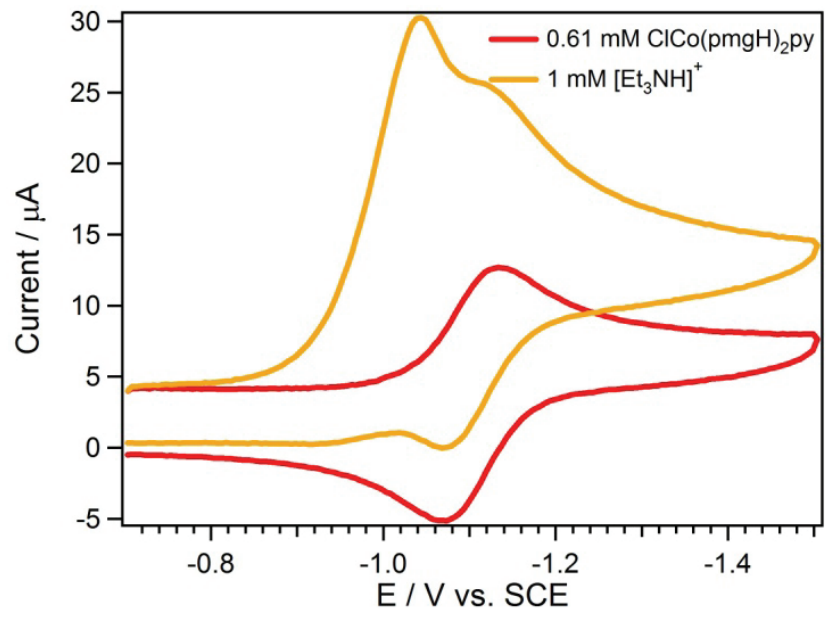

Figure 6.2 Cyclic voltammogram of $0.61 \mathrm{mM} \mathrm{ClCoIII(pmgH})_{2}$ py in $0.1 \mathrm{M}\left[\mathrm{NBu}_{4}\right]\left[\mathrm{PF}_{6}\right]$ acetonitrile solution in the absence and presence of $1 \mathrm{mM}$ [Et $\left.{ }_{3} \mathrm{~N}\right][\mathrm{OTs}]$. Scan rate of $100 \mathrm{mV} \mathrm{s}-1$. 


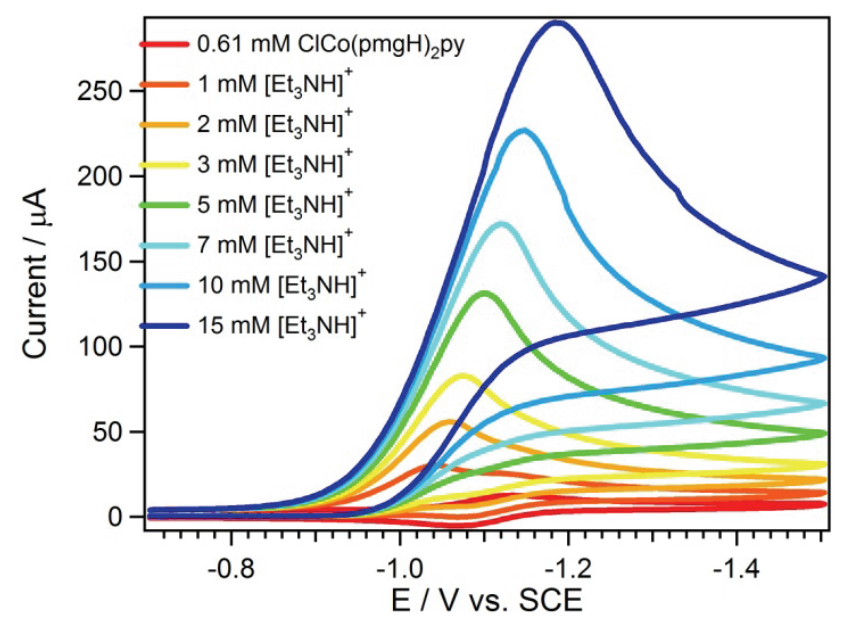

Figure 6.3 Cyclic voltammograms of $0.61 \mathrm{mM} \mathrm{ClCo}{ }^{\mathrm{III}}(\mathrm{pmgH})_{2} \mathrm{py}$ in $0.1 \mathrm{M}\left[\mathrm{NBu}_{4}\right]\left[\mathrm{PF}_{6}\right]$ acetonitrile solution in the presence of $\left[\mathrm{Et}_{3} \mathrm{~N}\right][\mathrm{OTs}]$. Scan rate of $100 \mathrm{mV} \mathrm{s}^{-1}$.

\section{Surface Functionalization}

Preliminary efforts to covalently attach $\mathrm{ClCo}^{\mathrm{III}}(\mathrm{pmgH})_{2}$ py are underway. Two approaches have been taken to prepare chemically modified electrodes. First, hydrosilylation was utilized to covalently link pentenyl-methylglyoxime directly to silicon. In this reaction, light activates the addition of a Si-H bond across an unsaturated $\mathrm{C}-\mathrm{C}$ bond (Scheme 6.3). While the surfaces functionalized via hydrosilylation are unlikely to be fully passivated, the route was explored for proof-of-concept.

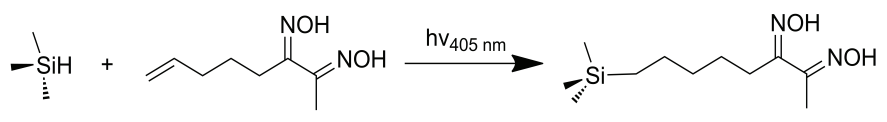

Scheme 6.3

A $0.2 \mathrm{M}$ solution of pentenyl-methylglyoxime was prepared in 1:1 acetonitrile:toluene. A freshly etched silicon ( $\mathrm{Si} 111$ ) wafer was placed in the ligand solution and the sample was 
irradiated with a $405 \mathrm{~nm}$ LED for 16 hours. X-ray photoelectron spectroscopy (XPS) was utilized to analyze the modified silicon surface. Based on the nitrogen peak, a 10-15\% surface coverage was estimated, but unfortunately a substantial amount of advantageous carbon was also noted, suggesting deleterious side reactions compete with the desired hydrosilylation reaction under these conditions.

Next, a route based on the secondary functionalization of a mixed monolayer on a $\operatorname{Si}(111)$ surface was explored (Scheme 6.4). As per the method of O'Leary and coworkers, $\operatorname{Si}(111)$ surfaces passivated with a methyl/thienyl monolayer were prepared..$^{10}$ Reaction with Nbromosuccinimide (NBS) provided the brominated thienyl. The Si wafer was then placed in a solution of $\mathrm{Pd}^{0}\left(\mathrm{PPh}_{3}\right)_{4}$, allowed to react, rinsed, and placed in a solution of pentenylmethylglyoxime to complete the Heck coupling reaction and yield the desired functionalization of the mixed monolayer. XPS measurements confirmed the presence of the nitrogen containing ligand with an estimated surface coverage of 9-15\%.

Work is currently underway to prepare the cobaloxime modified Si surfaces. These functionalized electrodes are expected to exhibit exceptional passivation towards oxidation and have low surface recombination velocities comparable to $\mathrm{CH}_{3}-\mathrm{Si}(111)$ surfaces, properties desirable for a robust photocathode material. ${ }^{9}$

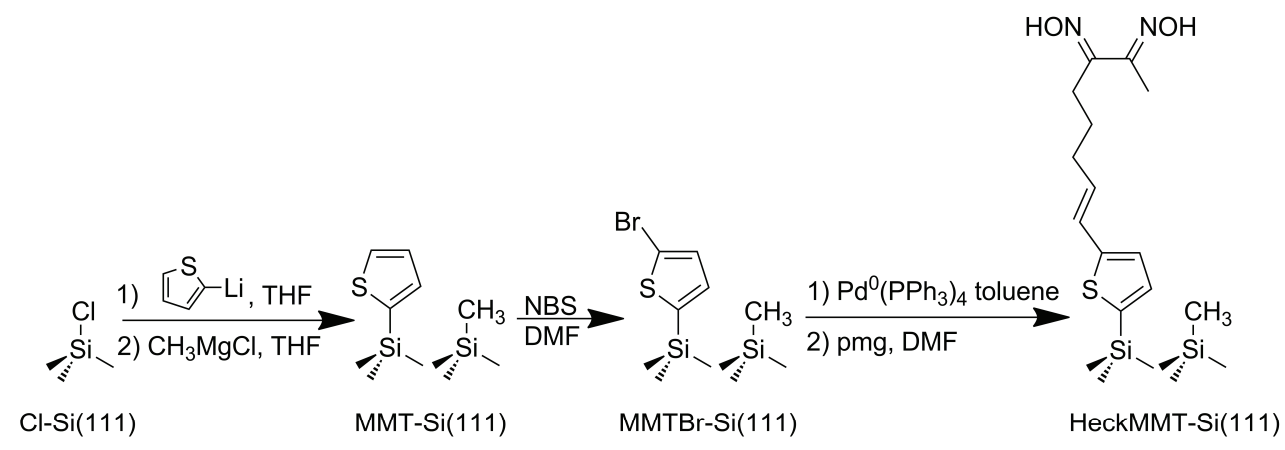


Conclusions

A cobaloxime with olefin functionality built into the glyoxime backbone has been prepared and is an active electrocatalyst for $\mathrm{H}_{2}$ evolution from weak acids. Work is underway to covalently link the catalyst to a passivated $\mathrm{Si}(111)$ surface to yield a cobaloxime modified electrode. Once obtained, the hydrogen evolving activity of the chemically modified electrode will be explored both electrochemically and photoelectrochemically.

\section{Acknowledgements}

These experiments were carried out in close collaboration with Kedy Edme, Chantal Mustoe, Leslie O’Leary, and Judy Lattimer.

\section{Experimental Details}

Synthesis

All reagents were purchased from chemical suppliers and used as received unless otherwise noted. ${ }^{1} \mathrm{H}$ NMR measurements were recorded on a Varian MERCURY $300 \mathrm{MHz}$ spectrometer and referenced to internal solvents

$\left[\mathbf{E t}_{3} \mathbf{N H}\right][\mathrm{OTs}]$ Preparation of triethylammonium tosylate $\left(\left[\mathrm{Et}_{3} \mathrm{NH}\right][\mathrm{OTs}]\right)$ was adapted from procedures for the ammonium ionic liquids reported by Wang et al. ${ }^{17} 2.53 \mathrm{~g}$ of triethylamine (25 mmol, 1 eq.) was placed in a two-neck round-bottom flask fitted with a reflux condenser and a addition funnel, and heated to $60{ }^{\circ} \mathrm{C} .4 .75 \mathrm{~g}$ of $p$-toluene sulfonic acid monohydrate (25 mmol, 1 eq.) was dissolved in $10 \mathrm{~mL}$ of water and added dropwise into the triethylamine at $60^{\circ} \mathrm{C}$ over the course of $1 \mathrm{~h}$. The reaction was then stirred for two hours at $80^{\circ} \mathrm{C}$. Excess water was removed by heating the solution to $80^{\circ} \mathrm{C}$ under vacuum to 
yield a pale tan solid. ${ }^{1} \mathrm{H}$ NMR $\left(300 \mathrm{MHz},\left(\mathrm{CD}_{3}\right)_{2} \mathrm{CO}\right): \delta 7.69(\mathrm{~d}, 2 \mathrm{H}, \mathrm{Ar}-\mathrm{H}), \delta 7.22(\mathrm{~d}$, 2H, Ar-H), $\delta 4.1\left(\mathrm{~s}, 3 \mathrm{H}, \mathrm{Ar}-\mathrm{CH}_{3}\right), \delta 3.23\left(\mathrm{~m}, 6 \mathrm{H}, \mathrm{CH}_{2}\right), \delta 2.35(\mathrm{~s}, 1 \mathrm{H}, \mathrm{N}-\mathrm{H}), \delta 1.32(\mathrm{t}, 9 \mathrm{H}$, $\left.\mathrm{CH}_{3}\right) \mathrm{ppm}$.

Pentenyl-methylglyoxime $125 \mathrm{~mL}$ of degassed 200 proof ethanol was transferred to a Schlenk flask containing sodium (1.62 g, $70.4 \mathrm{mmol}, 1.05$ eq.) under an argon atmosphere. Upon reaction to form sodium ethoxide, ethyl acetoacetate $(8.73 \mathrm{~g} 67.0 \mathrm{mmol}, 1 \mathrm{eq})$ was syringed into the reaction vessel and the mixture was stirred for 20 minutes. The reaction was then exposed to atmosphere and heated to $80-90{ }^{\circ} \mathrm{C}$. 5-bromo-1-pentene $(10 \mathrm{~g}, 67.0$ mmol, 1 eq.) was added and the reaction was stirred at reflux for $15 \mathrm{~h}$, during which a white solid crashed out. Ethanol was removed by rotary evaporation to yield a yellow oil containing a white precipitate $(\mathrm{NaBr})$. Enough water was added to the product to dissolve the precipitate, and the mixture was extracted with ether. The ether layers were combined and dried over anhydrous magnesium sulfate and ether was removed via rotary evaporation. Potassium hydroxide (5.64 g, $100 \mathrm{mmol}, 1.5 \mathrm{eq}$.$) in 60 \mathrm{~mL}$ of water was added to the resulting yellow oil and the mixture was allowed to stir for $2 \mathrm{~h}$ at room temperature until it was certain the hydrolysis reaction had gone to completion. The yellow solution was cooled to $5^{\circ} \mathrm{C}$ in an ice bath, and sodium nitrite $(4.63 \mathrm{~g}, 67 \mathrm{mmol}, 1 \mathrm{eq}$.) was added to the cooled solution. $10 \%$ sulfuric acid was added dropwise until the solution turned a potassium iodide/starch solution blue for at least 15 minutes. A solution of potassium hydroxide was added to the reaction until the $\mathrm{pH}$ was between 13 and 14 . The basic layer was extracted with ether to remove all unreacted materials. The basic layer was cooled and $4 \mathrm{M}$ sulfuric acid was added until the $\mathrm{pH}$ reached 4 . The solution was then extracted with ether and the yellow ether layers were combined and washed with $2 \mathrm{M}$ aqueous sodium carbonate solution, at which point the ether layer became almost colorless. The ether was removed via rotary evaporation to give $2 \mathrm{~g}$ of an oily yellow-white solid (20\%). The product 
was placed in a $500 \mathrm{~mL}$ round bottom flask, to which hydroxylamine hydrochloride (1.79 g, $24.6 \mathrm{mmol}, 2$ eq.), sodium carbonate (1.37 g, $12.3 \mathrm{mmol}, 1$ eq.), and $100 \mathrm{~mL}$ of ethanol were added. $40 \mathrm{~mL}$ of water was added to make the mixture homogeneous. The mixture was brought to reflux for $10 \mathrm{~h}$, then allowed to cool to room temperature. Most of the ethanol was removed by rotary evaporation, and the flask was placed in the fridge overnight, at which point a crystalline product crashed out. The solid product was collected via filtration, and thoroughly washed with water to remove any residual chloride ions, detected via reaction of a filtrate aliquot with $\mathrm{AgNO}_{3}$ in acetonitrile. The solid ligand was dried under vacuum. ${ }^{1} \mathrm{H}$ NMR (300 MHz, $\left.\left(\mathrm{CD}_{3}\right)_{2} \mathrm{CO}\right): \delta 10.48$ (s, $1 \mathrm{H}$, oxime), $10.45(\mathrm{~s}, 1 \mathrm{H}$, oxime), $5.82\left(\mathrm{~m}, 1 \mathrm{H},-\mathrm{CH}=\mathrm{CH}_{2}\right), 4.9\left(\mathrm{~m}, 2 \mathrm{H},-\mathrm{CH}=\mathrm{CH}_{2}\right), 2.62\left(\mathrm{~m}, 2 \mathrm{H}, \boldsymbol{\alpha}-\mathrm{CH}_{2}\right), 2.5(\mathrm{~m}, 2$ $\left.\mathrm{H}, \gamma-\mathrm{CH}_{2}\right), 2.26\left(\mathrm{~s}, 3 \mathrm{H}, \mathrm{CH}_{3}\right), 1.52\left(\mathrm{~m}, 2 \mathrm{H}, \beta-\mathrm{CH}_{2}\right) \mathrm{ppm}$.

ClCo(pmgH $)_{2}$ py Pentenyl-methylglyoxime (0.196 g, 1.15 mmol, 2 eq.) were added to $40 \mathrm{ml}$ of ethanol in a two-neck round bottom flask. The mixture was stirred and degassed under positive argon pressure for 20 minutes. The reaction was brought to $70^{\circ} \mathrm{C}$ and $2 \mathrm{~mL}$ of water was added to the mixture to facilitate the dissolution of the ligand. Under positive argon pressure, cobalt(II) chloride hexahydrate $(0.136 \mathrm{~g}, 0.575 \mathrm{mmol}, 1$ eq. $)$ was added to the mixture to form a light brown solution. The reaction was refluxed for 45 minutes at $80^{\circ}$ C, at which point pyridine $(0.091 \mathrm{~g}, 1.15 \mathrm{mmol}, 2$ eq. $)$ was syringed into the hot solution. The reaction was cooled to room temperature, and air was bubbled through the dark brown solution for six hours. Ethanol was removed by rotary evaporation to yield a brown oil, which was subsequently dissolved in $8 \mathrm{~mL}$ of chloroform and the organic fraction was decanted from residual water. The chloroform was removed by rotary evaporation to yield a dark brown oil, which was washed with $10 \mathrm{ml}$ of ether and placed under vacuum to yield a brown solid (0.143 g, 50\%). ${ }^{1} \mathrm{H}$ NMR (300 MHz, $\left.\left(\mathrm{CD}_{3}\right)_{2} \mathrm{CO}\right): \delta 18.6$ (s, $2 \mathrm{H}$, bridging oxime), 8.57 (m, $2 \mathrm{H}$, py $\mathrm{CH}(2,6)), 7.76(\mathrm{~m}, 2 \mathrm{H}$, py $\mathrm{CH}(3,5)), 7.34(\mathrm{~m}, 1 \mathrm{H}, \mathrm{py} \mathrm{CH}(4)), 5.75$ 
(m, 1H, $\left.-\mathrm{CH}=\mathrm{CH}_{2}\right), 4.9\left(\mathrm{~m}, 2 \mathrm{H},-\mathrm{CH}=\mathrm{CH}_{2}\right), 2.62\left(\mathrm{~m}, 2 \mathrm{H}, \mathrm{a}-\mathrm{CH}_{2}\right), 2.5\left(\mathrm{~m}, 2 \mathrm{H}, \gamma-\mathrm{CH}_{2}\right)$, $2.35\left(\mathrm{~s}, 3 \mathrm{H}, \mathrm{CH}_{3}\right), 1.52\left(\mathrm{~m}, 2 \mathrm{H}, \beta-\mathrm{CH}_{2}\right) \mathrm{ppm}$.

\section{Electrochemistry}

All electrochemical measurements were performed under anaerobic and anhydrous conditions. Dry acetonitrile was prepared by passing commercially available dry solvent over an activated alumina column and was stored on $4 \AA$ sieves under nitrogen. Tetrabutylammonium hexafluorophosphate was recrystallized from ethanol before use. Cyclic voltammograms were obtained using a Pine Instruments WaveNow potentiostat and the data were processed using Igor Pro 5.01 (Wavemetrics). Electrochemical analysis was carried out in a three-electrode cell, consisting of a glassy carbon working electrode (surface area $\left.=0.07 \mathrm{~cm}^{2}\right)$, a platinum wire counter electrode, and a $\mathrm{Ag} / \mathrm{AgNO}_{3}(0.01 \mathrm{M})$ reference electrode filled with $0.1 \mathrm{M}\left[{ }^{n} \mathrm{Bu}_{4}\right]\left[\mathrm{PF}_{6}\right]$ acetonitrile solution. All potentials were referenced to $\mathrm{Fc} / \mathrm{Fc}^{+}$as an internal standard and converted to $\mathrm{SCE}\left(\mathrm{E}^{\circ}\left(\mathrm{Fc} / \mathrm{Fc}^{+}\right)=0.380 \mathrm{~V}\right.$ vs. SCE$)$.

\section{Surface Chemistry}

XPS data were collected using a Surface Science Instruments M-Probe system. Ejected electrons were collected at an angle of $35^{\circ}$ from the surface normal, and the sample chamber was maintained at $<5 \times 10^{-9}$ Torr. Survey scans from 0 to $1000 \mathrm{eV}$ were performed to identify the elements present on the surface. High resolution spectra were collected to identify elements of interest, including $\mathrm{C}, \mathrm{N}, \mathrm{O}$. The XPS data were analyzed using the ESCA Data Analysis Application (V2.01.01; Service Physics, Bend, OR).

Preparation of Si(111) wafers: $\operatorname{Si}(111)$ wafers were cut to the desired size, rinsed sequentially with water, methanol, acetone, methanol, and water, then heated at $100{ }^{\circ} \mathrm{C}$ in piranha 
solution (1:3 10.1 $\mathrm{M} \mathrm{H}_{2} \mathrm{O}_{2}(\mathrm{aq}): 18 \mathrm{M} \mathrm{H}_{2} \mathrm{SO}_{4}$ ) for 10 minutes, then rinsed with water and dried. Hydrogen terminated Si samples were prepared by one of two methods reported by O'Leary et al. ${ }^{9}$ In one method, the clean wafer was placed in a buffered HF(aq) solution for 18 seconds, then rinsed with water, dried under a stream of $\mathrm{N}_{2}$, then placed in a degassed 11 $\mathrm{M} \mathrm{NH} \mathrm{NH}_{4} \mathrm{~F}$ solution for 17 minutes. During the etching process, the $\mathrm{NH}_{4} \mathrm{~F}$ solution was continuously purged with a stream of argon. Upon removal from the solution, the wafer was dried under a stream of $\mathrm{N}_{2}$ and immediately utilized or transferred to an inert atmosphere.

Hydrosilylation reaction: In a nitrogen filled glovebox, a $0.2 \mathrm{M}$ solution of pentenylmethylglyoxime was prepared in 1:1 acetonitrile:toluene. A freshly etched Si 111 wafer was placed in the ligand solution and the sample was irradiated with a $405 \mathrm{~nm}$ LED for 16 hours. The sample was then rinsed and sonicated subsequently in acetonitrile, methanol, and water and stored in an inert atmosphere until XPS measurements were carried out.

Heck coupling reaction: Preparation of the methyl/thienyl monolayers on $\mathrm{Si}(111)$ surfaces was carried out in a two-step halogenation/alkylation procedure developed by O'Leary et al. ${ }^{9-10}$ Heck coupling reactions were performed on these mixed monolayers via the method of O'Leary et al. ${ }^{10}$ In summary, reactions with N-bromosuccinimide (NBS), $\mathrm{Pd}^{0}\left(\mathrm{PPh}_{3}\right)_{4}$, and pentenyl-methylglyoxime were carried out sequentially, and the wafer was thoroughly rinsed between reaction steps, as described in Scheme 6.4. The functionalized wafer was analyzed via XPS. Based on the nitrogen-silicon ratio, surface coverage of $9-15 \%$ was estimated. 


\section{References}

1. Le Goff, A.; Artero, V.; Jousselme, B.; Tran, P. D.; Guillet, N.; Metaye, R.; Fihri, A.; Palacin, S.; Fontecave, M. Science 2009, 326, 1384.

2. Pantani, O.; Anxolabehere-Mallart, E.; Aukauloo, A.; Millet, P. Electrochem. Commun. 2007, 9, 54 .

3. Berben, L. A.; Peters, J. C. Chem. Commun. 2010, 46, 398.

4. Dominey, R. N.; Lewis, N. S.; Bruce, J. A.; Bookbinder, D. C.; Wrighton, M. S. J. Am. Chem. Soc. 1982, 104, 467.

5. Buriak, J. M. Chem. Rev. 2002, 102, 1271.

6. Royea, W. J.; Juang, A.; Lewis, N. S. Appl. Phys. Lett. 2000, 77, 1988.

7. Juang, A.; Scherman, O. A.; Grubbs, R. H.; Lewis, N. S. Langmuir 2001, 17, 1321.

8. Plass, K. E.; Liu, X.; Brunschwig, B. S.; Lewis, N. S. Chem. Mater. 2008, 20, 2228.

9. O’Leary, L. E.; Johansson, E.; Brunschwig, B. S.; Lewis, N. S. J. Phys. Chem. B 2010, null.

10. O’Leary, L. E.; Rose, M. J.; Ding, T.; Johannson, L. E.; Brunschwig, B. S.; Lewis, N. S. Unpublished Results.

11. Lance, K. A.; Goldsby, K. A.; Busch, D. H. Inorg. Chem. 1990, 29, 4537.

12. Schrauzer, G. N. Inorg. Synth. 1968, 11, 61.

13. Du, P.; Schneider, J.; Luo, G.; Brennessel, W. W.; Eisenberg, R. Inorg. Chem. 2009, 48, 4952.

14. Felton, G. A. N.; Glass, R. S.; Lichtenberger, D. L.; Evans, D. H. Inorg. Chem. 2006, 45, 9181.

15. Razavet, M.; Artero, V.; Fontecave, M. Inorg. Chem. 2005, 44, 4786.

16. Saveant, J.-M. Elements of Molecular and Biomolecular Electrochemistry; WileyInterscience: Hoboken, N.J., 2006. 
17. Wang, C.; Guo, L.; Li, H.; Wang, Y.; Weng, J.; Wu, L. Green Chem. 2006, 8, 603. 
A P P E N D I X A

PHOTOCHEMICAL GENERATION OF A POWERFUL OS(II) REDUCTANT

Portions of this work are excerpted with permission from:

Dempsey, J. L.; Winkler, J. R.; Gray, H. B. Submitted.

Unpublished Material Copyright 2010 American Chemical Society 


\section{Introduction}

Osmium(II) pentaammine complexes are potent reductants, but stable complexes can be formed with good $\pi$-acceptor ligands, such as $\mathrm{N}_{2}, \mathrm{CO}$, and $\mathrm{NO}^{+} \cdot{ }^{1-3}$ Ford and coworkers have demonstrated the dinitrogen ligand of $\left[\mathrm{Os}\left(\mathrm{NH}_{3}\right)_{5} \mathrm{~N}_{2}\right]^{2+}$ is photolabile. ${ }^{4}$ Excitation of aqueous solutions of $\left[\mathrm{Os}^{\mathrm{II}}\left(\mathrm{NH}_{3}\right)_{5} \mathrm{~N}_{2}\right]^{2+}$ between $229-365 \mathrm{~nm}$ leads to photoaquation, yielding $\left[\mathrm{Os}^{\mathrm{II}}\left(\mathrm{NH}_{3}\right)_{5} \mathrm{H}_{2} \mathrm{O}\right]^{2+}$, which is thermally oxidized by the solvent to yield Os(III) products (Scheme A.1). ${ }^{4-5}$ Light-driven population of ligand field states is likely the mechanism responsible mechanism for the photoaquation process. ${ }^{4,6}$

$\left[\mathrm{Os}\left(\mathrm{NH}_{3}\right)_{5} \mathrm{~N}_{2}\right]^{2+} \stackrel{\mathrm{hv}_{355 \mathrm{~nm}}}{\longrightarrow}\left[\mathrm{Os}\left(\mathrm{NH}_{3}\right)_{5}\right]^{2+} \stackrel{\mathrm{H}_{2} \mathrm{O}}{\longrightarrow}\left[\mathrm{Os}\left(\mathrm{NH}_{3}\right)_{5}\left(\mathrm{H}_{2} \mathrm{O}\right)\right]^{2+}$

\section{Scheme A.1}

The $\mathrm{p} K_{\mathrm{a}}$ of the photoaquation product, $\left[\mathrm{Os}^{\mathrm{II}}\left(\mathrm{NH}_{3}\right)_{5}\left(\mathrm{H}_{2} \mathrm{O}\right)\right]^{2+}$ has been estimated to lie between 10 and 12 , while the $\mathrm{p} K_{\mathrm{a}}$ of the corresponding Os(III) species is significantly lower, 4.85. ${ }^{5,7}$ At low $\mathrm{pH}$, the aquo species is protonated in both redox states and a reduction potential of $-0.97 \mathrm{~V}$ vs. SCE for $\left[\mathrm{Os}\left(\mathrm{NH}_{3}\right)_{5}\left(\mathrm{H}_{2} \mathrm{O}\right)\right]^{3+/ 2+}$ was reported by Gulens and Page. ${ }^{7-8}$ When the $\mathrm{pH}$ is raised above 4.85 , the formal potential varies with $\mathrm{pH}$, as predicted by the Nernst equation for a $1 \mathrm{e}^{-}, 1 \mathrm{H}^{+}$process, as the Os(II) species is protonated, while Os(III) is not. ${ }^{5}$ While electrochemical measurements for $\left[\mathrm{Os}\left(\mathrm{NH}_{3}\right)_{5}(\mathrm{OH})\right]^{2+/+}$ under strongly basic conditions have not been reported, extrapolation of the $\mathrm{pH}$ dependent data to $\mathrm{pH} 10$ and 12 yields estimated standard potentials for $\left[\mathrm{Os}\left(\mathrm{NH}_{3}\right)_{5}(\mathrm{OH})\right]^{2+/+}$ of ca. -1.27 and -1.39 , respectively.

In this work, we show that this photoinduced ligand exchange process can be utilized to phototrigger the generation of a powerful reductant, whose reactivity can be monitored 
easily with time-resolved spectroscopy. Methyl viologen $\left(\mathrm{MV}^{2+}, \mathrm{E}^{0}\left(\mathrm{MV}^{2+\circ+}\right)=-0.69 \mathrm{~V}\right.$ vs. SCE in $\left.\mathrm{H}_{2} \mathrm{O}\right)^{9}$ acts as an electron acceptor, and the appearance of the vivid blue color indicative of the radical cation $\left(\mathrm{MV}^{\cdot+}\right)$ formed upon reduction is easily tracked with transient absorption spectroscopy (Scheme A.2). This chemistry is accessible in both aqueous media and acetonitrile solution.

$$
\left[\mathrm{Os}\left(\mathrm{NH}_{3}\right)_{5}\left(\mathrm{H}_{2} \mathrm{O}\right)\right]^{2+}+\mathrm{MV}^{2+} \longrightarrow\left[\mathrm{Os}\left(\mathrm{NH}_{3}\right)_{5}\left(\mathrm{H}_{2} \mathrm{O}\right)\right]^{3+}+\mathrm{MV}^{\bullet+}
$$

Scheme A.2

\section{Results and Discussion}

Photolysis of a sample containing $\left[\mathrm{Os}^{\mathrm{II}}\left(\mathrm{NH}_{3}\right)_{5} \mathrm{~N}_{2}\right] \mathrm{Cl}_{2}$ and $[\mathrm{MV}] \mathrm{Cl}_{2}$ in neutral buffered aqueous solution $(100 \mathrm{mM} \mathrm{NaPi})$ led to the irreversible formation of $\mathrm{MV}^{\bullet+}\left(\lambda_{\mathrm{ex}}=355 \mathrm{~nm}\right)$. The growth of new absorption features at 393 and $605 \mathrm{~nm}$, characteristic of the viologen radical, is seen in absorption spectra measured at arbitrary intervals during photolysis (Figure A.1). ${ }^{10}$ Similar behavior was observed at other $\mathrm{pHs.}$

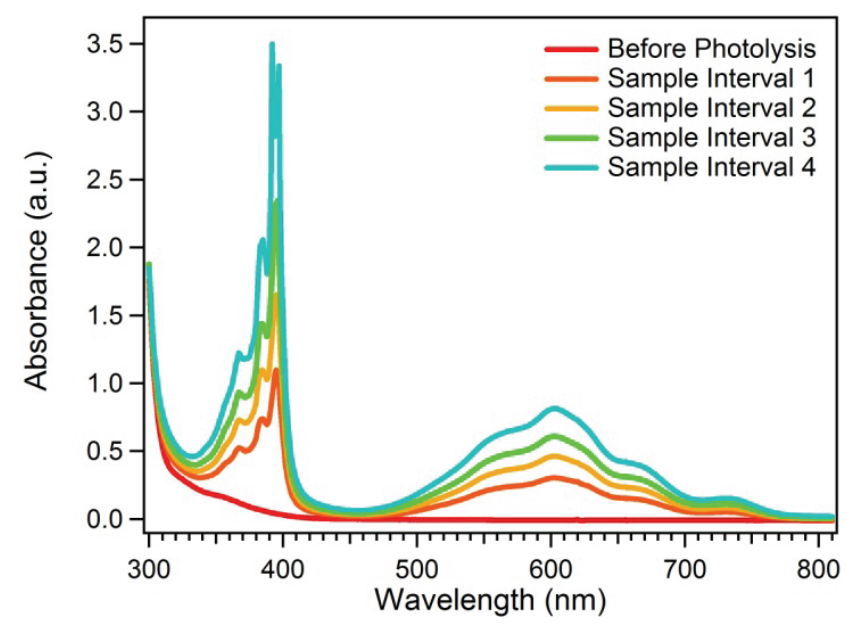

Figure A.1 Absorption spectra measured at arbitrary intervals upon photolysis at $355 \mathrm{~nm}$ in aqueous solution. Sample conditions: $1.6 \mathrm{mM}\left[\mathrm{Os}\left(\mathrm{NH}_{3}\right)_{5} \mathrm{~N}_{2}\right] \mathrm{Cl}_{2}, 1.2 \mathrm{mM}[\mathrm{MV}] \mathrm{Cl}_{2}, 100 \mathrm{mM}$ $\mathrm{NaP} i, \mathrm{pH} 7$. 
The appearance of the reduced viologen was monitored via transient absorption $\left(\lambda_{\text {obs }}=\right.$ $610 \mathrm{~nm}$ ) to determine the rate of electron transfer from the photogenerated Os(II) aquo species to $\mathrm{MV}^{2+}$ (Figure A.2). The formation of $\mathrm{MV}^{\bullet+}$ is best fit to single exponential kinetics, and the first-order rate constant is linearly dependent on the concentration of $\mathrm{MV}^{2+}$ under these pseudo-first-order conditions (methyl viologen in excess), allowing second-order rate constants for electron transfer to be determined.

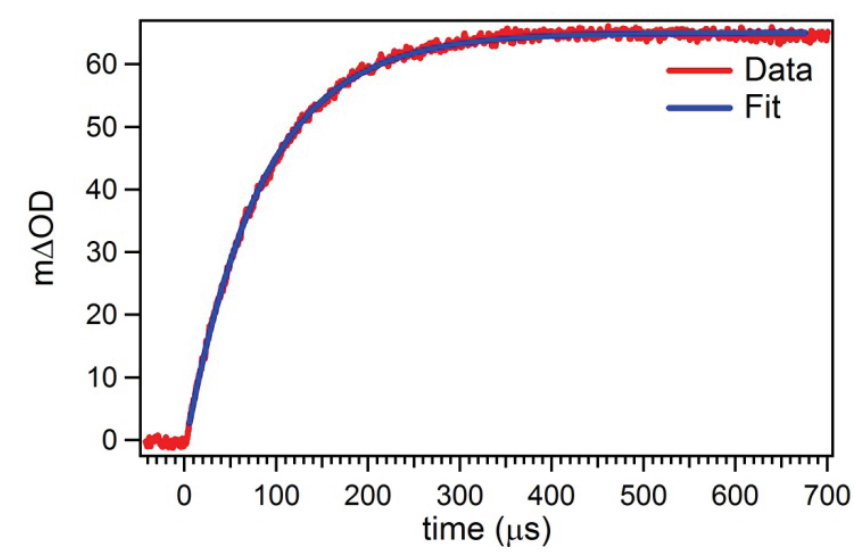

Figure A.2 Transient kinetics trace monitoring the appearance of $\mathrm{MV}^{\bullet+}$ upon photochemical generation of $\left[\mathrm{Os}\left(\mathrm{NH}_{3}\right)_{5}\left(\mathrm{H}_{2} \mathrm{O}\right)\right]^{2+}\left(\lambda_{\text {ex }}\right.$ $\left.=355 \mathrm{~nm}, \lambda_{\text {obs }}=610 \mathrm{~nm}\right)$ fit to single exponential kinetics. Conditions: $1.7 \mathrm{mM}\left[\mathrm{Os}\left(\mathrm{NH}_{3}\right)_{5} \mathrm{~N}_{2}\right] \mathrm{Cl}_{2}, 0.36 \mathrm{mM}[\mathrm{MV}] \mathrm{Cl}_{2}, 100$ $\mathrm{mM} 100 \mathrm{mM} \mathrm{NaPi}, \mathrm{pH} 3$.

At $\mathrm{pH}$, when the aquo species is protonated in both the Os(II) and Os(III) oxidation states, a driving force of ca. $280 \mathrm{mV}$ can be estimated for electron transfer from $\left[\mathrm{Os}^{\mathrm{II}}\left(\mathrm{NH}_{3}\right)_{5}\left(\mathrm{H}_{2} \mathrm{O}\right)\right]^{2+}$ to $\mathrm{MV}^{2+}$ based on the reduction potentials for the species involved. A second-order rate constant for this electron transfer reaction of $3.2 \times 10^{7} \mathrm{M}^{-1} \mathrm{~s}^{-1}$ was determined (Figure A.3). 


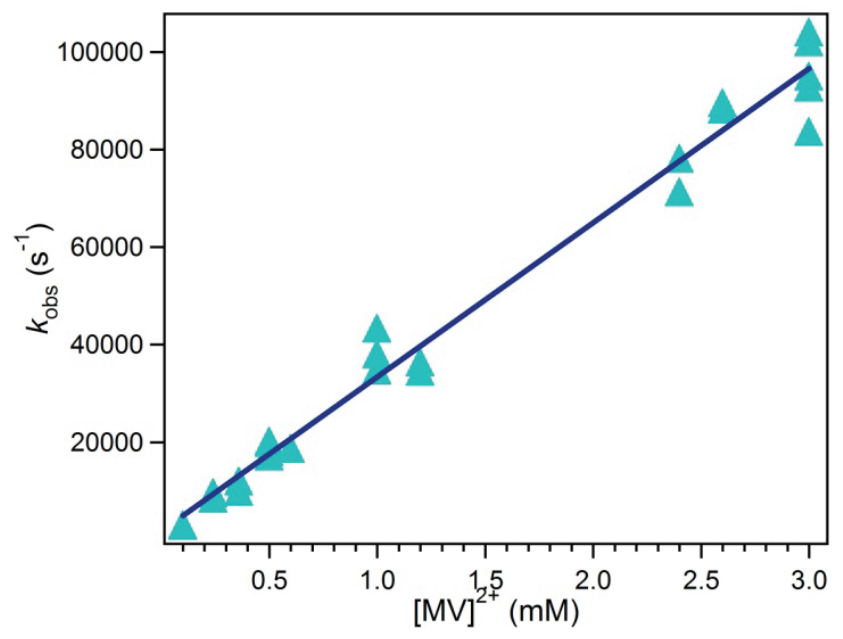

Figure A.3 First-order rate constants for the formation of $\mathrm{MV}^{\bullet+}$ upon electron transfer from $\left[\mathrm{Os}\left(\mathrm{NH}_{3}\right)_{5}\left(\mathrm{H}_{2} \mathrm{O}\right)\right]^{2+}$ to $\mathrm{MV}^{2+}$ at $\mathrm{pH} 3$. A linear relationship between the first-order rate constants and methyl viologen concentrations was found, allowing a secondorder rate constant $\left(k_{\mathrm{ET}}\right)$ for electron transfer to be determined. $k_{\mathrm{obs}}=k_{0}+k_{E T}\left[\mathrm{MV}^{2+}\right], k_{0}=1.8 \times 10^{3} \mathrm{~s}^{-1}, k_{\mathrm{ET}}=3.2 \times 10^{7} \mathrm{M}^{-1} \mathrm{~s}^{-1}$. Sample conditions: $1.7-3.5 \mathrm{mM} \quad\left[\mathrm{Os}\left(\mathrm{NH}_{3}\right)_{5} \mathrm{~N}_{2}\right] \mathrm{Cl}_{2}$, varying concentrations of $\left[\mathrm{MV}^{-} \mathrm{Cl}_{2}, 100 \mathrm{mM} \mathrm{NaPi}\right.$, pH 3 .

At $\mathrm{pH}$ 12, photolysis of $\left[\mathrm{Os}^{\mathrm{II}}\left(\mathrm{NH}_{3}\right)_{5} \mathrm{~N}_{2}\right]^{2+}$ yields the osmium(II) hydroxo species, $\left[\mathrm{Os}^{\mathrm{II}}\left(\mathrm{NH}_{3}\right)_{5}(\mathrm{OH})\right]^{+}$, a significantly more powerful reductant than $\left[\mathrm{Os}^{\mathrm{II}}\left(\mathrm{NH}_{3}\right)_{5}\left(\mathrm{H}_{2} \mathrm{O}\right)\right]^{2+}$, and the driving force for electron transfer from $\left[\mathrm{Os}^{\mathrm{II}}\left(\mathrm{NH}_{3}\right)_{5}(\mathrm{OH})\right]^{+}$to $\mathrm{MV}^{2+}$ is ca. $580-700 \mathrm{mV}$. The second-order rate constant obtained, $2.5 \times 10^{8} \mathrm{M}^{-1} \mathrm{~s}^{-1}$, is an order of magnitude larger than that obtained for the aquo species measured at $\mathrm{pH} 3$, as would be expected based on the $>300 \mathrm{mV}$ increase of driving force for electron transfer (Figure A.4). 


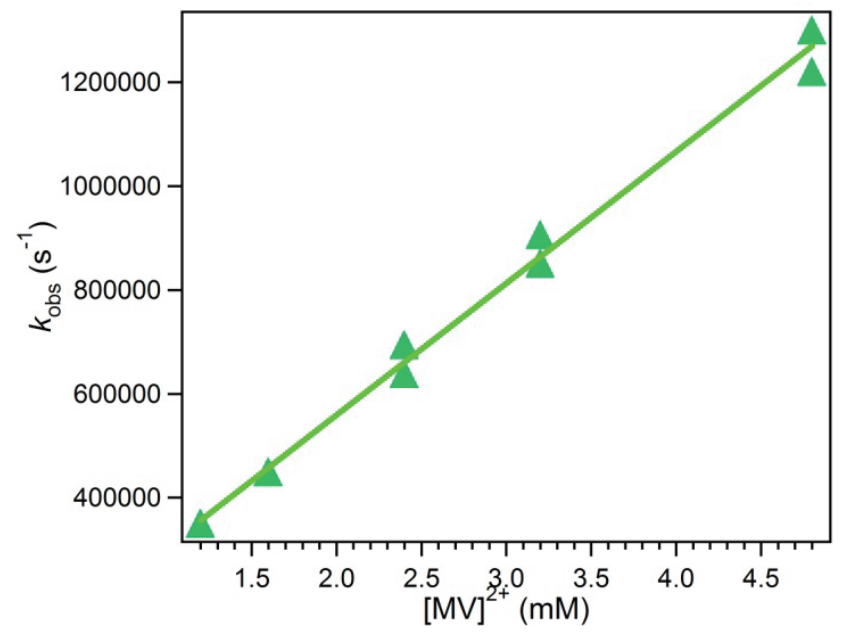

Figure A.4 First-order rate constants for the formation of $\mathrm{MV}^{\bullet+}$ upon electron transfer from $\left[\mathrm{Os}\left(\mathrm{NH}_{3}\right)_{5}(\mathrm{OH})\right]^{+}$to $\mathrm{MV}^{2+}$ at $\mathrm{pH} 12$. A linear relationship between the first-order rate constants and methyl viologen concentrations was found, allowing a secondorder rate constant $\left(k_{\mathrm{ET}}\right)$ for electron transfer to be determined. $k_{\mathrm{obs}}=k_{0}+k_{E T}\left[\mathrm{MV}^{2+}\right], k_{0}=5.5 \times 10^{4} \mathrm{~s}^{-1}, k_{\mathrm{ET}}=2.5 \times 10^{8} \mathrm{M}^{-1} \mathrm{~s}^{-1}$. Sample conditions: $2.2 \mathrm{mM} \quad\left[\mathrm{Os}\left(\mathrm{NH}_{3}\right)_{5} \mathrm{~N}_{2}\right] \mathrm{Cl}_{2}$, varying concentrations of $\left[\mathrm{MV}_{\mathrm{Cl}}, 100 \mathrm{mM} \mathrm{NaP} i, \mathrm{pH} 12\right.$.

At neutral $\mathrm{pH}$, an electron transfer rate constant of $9.3 \times 10^{7} \mathrm{M}^{-1} \mathrm{~s}^{-1}$ was determined (Figure A.5). While proton loss is expected to accompany electron transfer from $\left[\mathrm{Os}^{\mathrm{II}}\left(\mathrm{NH}_{3}\right)_{5}\left(\mathrm{H}_{2} \mathrm{O}\right)\right]^{2+}$ to form $\left[\mathrm{Os}^{\mathrm{III}}\left(\mathrm{NH}_{3}\right)_{5}(\mathrm{OH})\right]^{2+}$ at $\mathrm{pH} 7$, a $\mathrm{pH}$ dependence on the electron transfer rate constant was not expected. Likely, the basic component of the buffer, $\mathrm{HPO}_{4}{ }^{2-}$, acts as a proton acceptor, influencing the observed kinetics as seen in previous systems. ${ }^{11-13}$ Finklea and Savéant have previously explored the mechanisms of proton-coupled electron transfer processes in other $\mathrm{Os}^{\mathrm{II}}\left(\mathrm{H}_{2} \mathrm{O}\right) / \mathrm{Os}^{\mathrm{III}}(\mathrm{OH})$ systems. ${ }^{14-17}$ While a detailed examination of this observed phenomenon is outside the scope of this work, future studies will focus on studying the buffer and $\mathrm{pH}$ dependence of $\left[\mathrm{Os}\left(\mathrm{NH}_{3}\right)_{5}\left(\mathrm{H}_{2} \mathrm{O}\right)\right]^{2+}$ oxidation photochemically, studies which will complement those of Finklea and Savéant. 


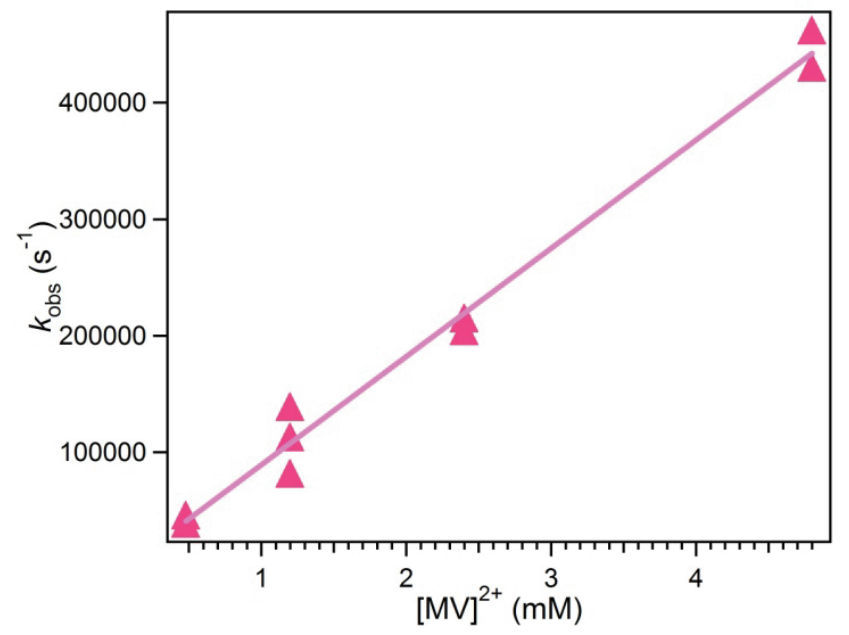

Figure A.5 First-order rate constants for the formation of $\mathrm{MV}^{\bullet+}$ upon electron transfer from $\left[\mathrm{Os}\left(\mathrm{NH}_{3}\right)_{5}(\mathrm{OH})\right]^{+}$to $\mathrm{MV}^{2+}$ at $\mathrm{pH}$ 7. A linear relationship between the first-order rate constants and methyl viologen concentrations was found, allowing a secondorder rate constant $\left(k_{\mathrm{ET}}\right)$ for electron transfer to be determined. $k_{\mathrm{obs}}=k_{0}+k_{E T}\left[\mathrm{MV}^{2+}\right], k_{0}=-3.8 \times 10^{3} \mathrm{~s}^{-1}, k_{\mathrm{ET}}=9.3 \times 10^{7} \mathrm{M}^{-1} \mathrm{~s}^{-1}$. Sample conditions: $3.5 \mathrm{mM} \quad\left[\mathrm{Os}\left(\mathrm{NH}_{3}\right)_{5} \mathrm{~N}_{2}\right] \mathrm{Cl}_{2}$, varying concentrations of $\left[\mathrm{MV}^{-} \mathrm{Cl}_{2}, 100 \mathrm{mM} \mathrm{NaPi}\right.$, pH 7 .

Osmium(III) pentaammine nitrile complexes, $\left[\mathrm{Os}^{\mathrm{III}}\left(\mathrm{NH}_{3}\right)_{5}(\mathrm{RCN})\right]^{3+}$, have reduction potentials that occur significantly positive to those of the corresponding aquo species. A reduction potential of $-0.54 \mathrm{~V}$ vs. SCE was measured by Taube and coworkers for $\left[\mathrm{Os}^{\mathrm{III}}\left(\mathrm{NH}_{3}\right)_{5}\left(\mathrm{CH}_{3} \mathrm{CN}\right)\right]^{3+}$ in glyme, indicating that $\left[\mathrm{Os}^{\mathrm{II}}\left(\mathrm{NH}_{3}\right)_{5}\left(\mathrm{CH}_{3} \mathrm{CN}\right)\right]^{2+}$ can still drive the reduction of $\mathrm{MV}^{2+}\left(\mathrm{E}^{0}\left(\mathrm{MV}^{2+/ \bullet+}\right)=-0.46 \mathrm{~V}\right.$ vs. $\mathrm{SCE}$ in $\mathrm{CH}_{3} \mathrm{CN}$, Scheme A.3). ${ }^{18-19}$ Photolysis of $\left[\mathrm{Os}^{\mathrm{II}}\left(\mathrm{NH}_{3}\right)_{5} \mathrm{~N}_{2}\right]\left[\mathrm{PF}_{6}\right]_{2}$ in dry acetonitrile in the presence of $[\mathrm{MV}]\left[\mathrm{PF}_{6}\right]_{2}$ leads to the formation of the viologen radical cation, as seen in the steady-state absorption spectra measured at intervals of photolysis (Figure A.6).

$$
\begin{aligned}
& {\left[\mathrm{Os}\left(\mathrm{NH}_{3}\right)_{5} \mathrm{~N}_{2}\right]^{2+} \frac{\mathrm{hv}_{355 \mathrm{~nm}}}{\mathrm{CH}_{3} \mathrm{CN}}\left[\mathrm{Os}\left(\mathrm{NH}_{3}\right)_{5}\left(\mathrm{CH}_{3} \mathrm{CN}\right)\right]^{2+}} \\
& {\left[\mathrm{Os}\left(\mathrm{NH}_{3}\right)_{5}\left(\mathrm{CH}_{3} \mathrm{CN}\right)\right]^{2+}+\mathrm{MV}^{2+} \longrightarrow\left[\mathrm{Os}\left(\mathrm{NH}_{3}\right)_{5}\left(\mathrm{CH}_{3} \mathrm{CN}\right)\right]^{3+}+\mathrm{MV}^{\bullet+}}
\end{aligned}
$$




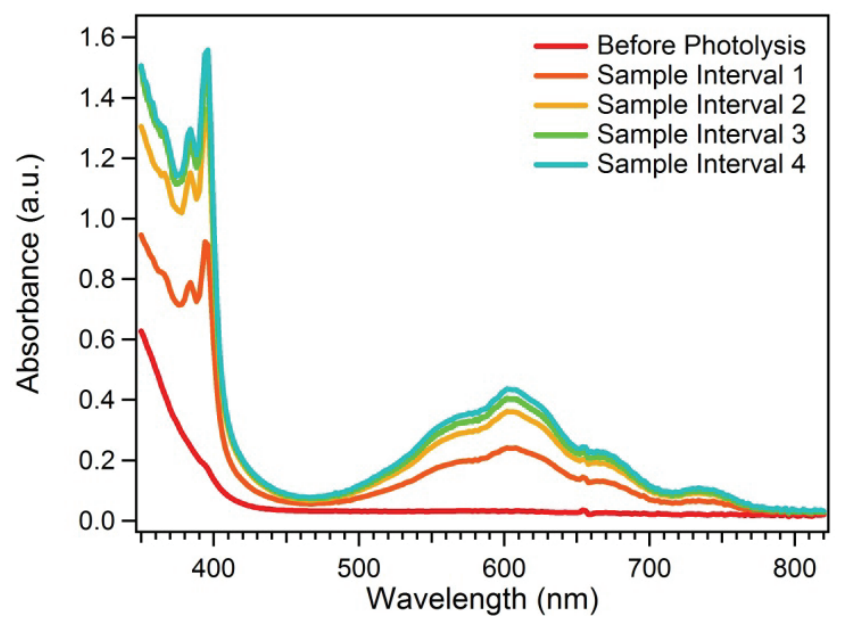

Figure A.6 Absorption spectra measured at arbitrary intervals upon photolysis at $355 \mathrm{~nm}$ in acetonitrile. Sample conditions: 3.5 $\mathrm{mM} \quad\left[\mathrm{Os}\left(\mathrm{NH}_{3}\right)_{5} \mathrm{~N}_{2}\right]\left[\mathrm{PF}_{6}\right]_{2}, \quad 1.2 \mathrm{mM} \quad[\mathrm{MV}]\left[\mathrm{PF}_{6}\right]_{2}, \quad 100 \mathrm{mM}$ $\left[{ }^{n} \mathrm{Bu}_{4} \mathrm{~N}\right]\left[\mathrm{PF}_{6}\right]$.

The driving force for reduction of $[\mathrm{MV}]\left[\mathrm{PF}_{6}\right]_{2}$ by $\left[\mathrm{Os}^{\mathrm{II}}\left(\mathrm{NH}_{3}\right)_{5}\left(\mathrm{CH}_{3} \mathrm{CN}\right)\right]^{2+}$ is significantly lower than those for the corresponding aquo and hydroxo species (ca. $80 \mathrm{mV}$ ). As such, a significantly smaller rate constant for electron transfer was determined, $4.9 \times 10^{4} \mathrm{M}^{-1} \mathrm{~s}^{-1}$ (Figure A.7). 


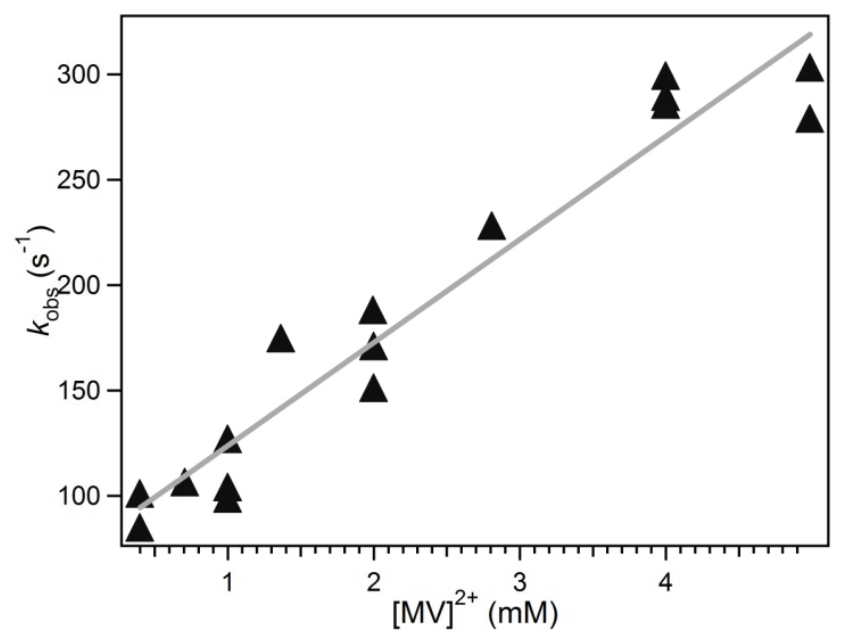

Figure A.7 First-order rate constants for the formation of $\mathrm{MV}^{\bullet+}$ upon electron transfer from $\left[\mathrm{Os}\left(\mathrm{NH}_{3}\right)_{5}\left(\mathrm{CH}_{3} \mathrm{CN}\right)\right]^{2+}$ to $\mathrm{MV}^{2+}$ in acetonitrile. A linear relationship between the first-order rate constants and methyl viologen concentrations was found, allowing a second-order rate constant $\left(k_{\mathrm{ET}}\right)$ for electron transfer to be determined. $k_{\mathrm{obs}}=k_{0}+k_{\mathrm{ET}}\left[\mathrm{MV}^{2+}\right], k_{0}=75 \mathrm{~s}^{-1}, k_{\mathrm{ET}}=4.9 \times 10^{4} \mathrm{M}^{-1}$ $\mathrm{s}^{-1}$. Sample conditions: $3.5 \mathrm{mM}\left[\mathrm{Os}\left(\mathrm{NH}_{3}\right)_{5} \mathrm{~N}_{2}\right]\left[\mathrm{PF}_{6}\right]_{2}$, varying concentrations of $[\mathrm{MV}]\left[\mathrm{PF}_{6}\right]_{2}, 100 \mathrm{mM}\left[{ }^{n} \mathrm{Bu}_{4} \mathrm{~N}\right]\left[\mathrm{PF}_{6}\right]$.

\section{Conclusions}

Photolysis of Os(II) dinitrogen species leads to the labilization of the $\mathrm{N}_{2}$ ligand. In coordinating solvents such as water and acetonitrile, ligand exchange occurs to form $\left[\mathrm{Os}^{\mathrm{II}}\left(\mathrm{NH}_{3}\right)_{5} \mathrm{~L}\right]^{2+}\left(\mathrm{L}=\mathrm{H}_{2} \mathrm{O}, \mathrm{CH}_{3} \mathrm{CN}\right)$, a powerful reductant. In the presence of methyl viologen, electron transfer from the Os(II) species forms the methyl viologen radical cation and $\left[\mathrm{Os}^{\mathrm{III}}\left(\mathrm{NH}_{3}\right)_{5} \mathrm{~L}\right]^{3+}$. At $\mathrm{pH} 12$, the large driving force $(\sim 580-700 \mathrm{mV})$ for electron transfer from the hydroxo species, $\left[\mathrm{Os}^{\mathrm{II}}\left(\mathrm{NH}_{3}\right)_{5}(\mathrm{OH})\right]^{+}$to $\mathrm{MV}^{2+}$ leads to a large rate constant of $2.5 \mathrm{x}$ $10^{8} \mathrm{M}^{-1} \mathrm{~s}^{-1}$ for the reaction. When photogenerated $\mathrm{Os}(\mathrm{II})$ aquo species is protonated $(\mathrm{pH}=$ 3), the driving force is attenuated (ca. $280 \mathrm{mV}$ ) and the rate constant for electron transfer to methyl viologen is an order of magnitude smaller than that for the hydroxo species, $3.2 \mathrm{x}$ $10^{7} \mathrm{M}^{-1} \mathrm{~s}^{-1}$. At neutral $\mathrm{pH}$, electron transfer is accompanied by deprotonation of the aquo 
ligand; under the conditions explored, the basic form of the buffer aids in this process, and a slight acceleration in rate is observed, as compared to the low $\mathrm{pH}$ value $\left(k_{\mathrm{ET}}=9.3 \mathrm{x}\right.$ $\left.10^{7} \mathrm{M}^{-1} \mathrm{~s}^{-1}\right)$. In acetonitrile, the driving force for electron transfer from the Os(II) species to $\mathrm{MV}^{2+}$ is only $80 \mathrm{mV}$, and this is reflected in the relatively small rate constant for this reaction $\left(4.9 \times 10^{4} \mathrm{M}^{-1} \mathrm{~s}^{-1}\right)$.

Ultimately, we have shown that powerful $\left[\mathrm{Os}^{\mathrm{II}}\left(\mathrm{NH}_{3}\right)_{5} \mathrm{~L}\right]^{2+}$ reductants can be generated upon photolysis of $\left[\mathrm{Os}^{\mathrm{II}}\left(\mathrm{NH}_{3}\right)_{5} \mathrm{~N}_{2}\right]^{2+}$ in coordinating solvents $\left(\mathrm{L}=\mathrm{H}_{2} \mathrm{O}, \mathrm{CH}_{3} \mathrm{CN}\right)$. Electron transfer to methyl viologen readily occurs, and the formation of the viologen radical can be monitored with transient absorption spectroscopy. Like the widely employed flash-quench techniques utilized by photochemists for time-resolved spectroscopic studies, the photochemical generation of these powerful Os(II) reductants should be useful for mechanistic investigations of reactions triggered by electron transfer. ${ }^{20-22}$ Further, these complexes are an intriguing platform with which to photochemically examine the mechanism of proton-coupled electron transfer from $\left[\mathrm{Os}^{\mathrm{II}}\left(\mathrm{NH}_{3}\right)_{5}\left(\mathrm{H}_{2} \mathrm{O}\right)\right]^{2+}$ to produce $\left[\mathrm{Os}^{\mathrm{III}}\left(\mathrm{NH}_{3}\right)_{5}(\mathrm{OH})\right]^{2+}$.

\section{Experimental Details}

\section{Reagents}

Tetrabutylammonium hexafluorophosphate was recrystallized from ethanol. $\left[\mathrm{Os}\left(\mathrm{NH}_{3}\right)_{5} \mathrm{~N}_{2}\right] \mathrm{Cl}_{2}$ obtained commercially from Aldrich and recrystallized from $\mathrm{HCl}$ per the method of Allen and Stevens and washed with diethyl ether. ${ }^{2}$ The hexafluorophosphate salts of $\left[\mathrm{Os}\left(\mathrm{NH}_{3}\right)_{5} \mathrm{~N}_{2}\right]^{2+}$ and $\mathrm{MV}^{2+}$ were prepared from the corresponding chloride salts via salt metathesis in water $\left(0^{\circ} \mathrm{C}\right)$, then washed with diethyl ether and dried under vacuum. 
$100 \mathrm{mM} \mathrm{NaPi}$ buffers were prepared accordingly from $\mathrm{H}_{3} \mathrm{PO}_{4}, \mathrm{NaH}_{2} \mathrm{PO}_{4}, \mathrm{Na}_{2} \mathrm{HPO}_{4}$ and adjusted to $\mathrm{pH} 3,7$, or 12 with $\mathrm{NaOH}$.

Aqueous samples were prepared and placed into the cell of a high-vacuum $1 \mathrm{~cm}$ pathlength fused quartz cuvette (Starna Cells) isolated from atmosphere by a high-vacuum Teflon valve (Kontes). Samples were boil-degassed and backfilled with argon 10 times to remove all oxygen from the solution.

Acetonitrile samples were prepared in a nitrogen filled glove box with acetonitrile that had been deoxygenated and dried by thorough sparging with $\mathrm{N}_{2}$ followed by passage through an activated alumina column. Samples were placed into the cell of a high-vacuum $1 \mathrm{~cm}$ pathlength fused quartz cuvette (Starna Cells) and isolated from atmosphere and the bulb by a high-vacuum Teflon valve (Kontes).

\section{Physical Methods}

UV-visible absorption measurements were carried out using a Hewlett Packard 8452 UVVis spectrophotometer in $1 \mathrm{~cm}$ pathlength quartz cuvettes.

Time-resolved spectroscopic measurements were carried out at the Beckman Institute Laser Resource Center (California Institute of Technology). $355 \mathrm{~nm}$ laser excitation was provided by $8 \mathrm{~ns}$ pulses from the third harmonic of a $10 \mathrm{~Hz}$ Q-switched Nd:YAG laser (SpectraPhysics Quanta-Ray PRO-Series). Probe light for transient absorption kinetics measurements was provided by a $75 \mathrm{~W}$ arc lamp (PTI Model A 1010) that could be operated in continuous wave or pulsed modes. Probe light was passed through a $550 \mathrm{~nm}$ long-pass filter prior to passing through the sample. Timing between the laser and the probe light was controlled by a digital delay generator. After passing through the sample collinearly with the laser beam, scattered excitation light was rejected by suitable long pass 
filters, and probe wavelengths were selected for detection by a double monochromator (Instruments SA DH-10) with $1 \mathrm{~mm}$ slits. Transmitted light was detected with a photomultiplier tube (PMT, Hamamatsu R928). The PMT current was amplified and recorded with a GageScope transient digitizer. The data were converted to units of $\Delta \mathrm{OD}$ $\left(\Delta \mathrm{OD}=-\log _{10}\left(\mathrm{I} / \mathrm{I}_{0}\right)\right.$, where $\mathrm{I}$ is the time-resolved probe-light intensity with laser excitation, and $I_{0}$ is the intensity without excitation). Samples measured on the microsecond timescale or faster were stirred continuously and measured using a laser repetition rate of $10 \mathrm{~Hz}$, while samples measured on a millisecond timescale were excited with a single shutterreleased laser pulse, stirred for $1 \mathrm{~s}$ after collecting data, then allowed to sit until the solution settled ( 2 s) before the next laser pulse. Data were averaged over approximately 15-100 shots. All instruments and electronics in these systems were controlled by software written in LabVIEW (National Instruments). Data were log-time compressed prior to fitting. Data manipulation was performed with MATLAB R2008a (Mathworks, Inc.) and graphed with Igor Pro 5.01 (Wavemetrics).

\section{References}

1. Allen, A. D.; Stevens, J. R. Chem. Commun. 1967, 1147.

2. Allen, A. D.; Stevens, J. R. Can. J. Chem. 1972, 50, 3093.

3. Elson, C. M.; Gulens, J.; Itzkovitch, I. J.; Page, J. A. Chem. Commun. 1970, 875.

4. Matsubara, T.; Bergkamp, M.; Ford, P. C. Inorg. Chem. 1978, 17, 1604.

5. Shi, C.; Anson, F. C. Inorg. Chem. 1997, 36, 2682.

6. Matsubara, T.; Ford, P. C. Inorg. Chem. 1978, 17, 1747.

7. Gulens, J.; Page, J. A. J. Electroanal. Chem. 1974, 55, 239.

8. Gulens, J.; Page, J. A. J. Electroanal. Chem. 1976, 67, 215.

9. Bird, C. L.; Kuhn, A. T. Chem. Soc. Rev. 1981, 10, 49. 
10. Creutz, C. Comment Inorg. Chem. 1982, 1, 293.

11. Irebo, T.; Reece Steven, Y.; Sjödin, M.; Nocera Daniel, G.; Hammarström, L. J. Am. Chem. Soc. 2007, 129, 15462.

12. Fecenko, C. J.; Meyer, T. J.; Thorp, H. H. J. Am. Chem. Soc. 2006, 128, 11020.

13. Costentin, C.; Robert, M.; Savéant, J.-M. J. Am. Chem. Soc. 2007, 129, 5870.

14. Haddox, R. M.; Finklea, H. O. J. Phys. Chem. B 2004, 108, 1694.

15. Madhiri, N.; Finklea, H. O. Langmuir 2006, 22, 10643.

16. Costentin, C.; Robert, M.; Saveant, J.-M.; Teillout, A.-L. ChemPhysChem 2009, 10, 191.

17. Costentin, C.; Robert, M.; Saveant, J.-M.; Teillout, A.-L. Proc. Natl. Acad. Sci. U. S. A. 2009, 106, 11829.

18. Sekine, M.; Harman, W. D.; Taube, H. Inorg. Chem. 1988, 27, 3604.

19. Chen, P.; Curry, M.; Meyer, T. J. Inorg. Chem. 1989, 28, 2271.

20. Ogata, T.; Yanagida, S.; Brunschwig, B. S.; Fujita, E. J. Am. Chem. Soc. 1995, 117, 6708.

21. Bjerrum, M. J.; Casimiro, D. R.; Chang, I. J.; Dibilio, A. J.; Gray, H. B.; Hill, M. G.; Langen, R.; Mines, G. A.; Skov, L. K.; Winkler, J. R.; Wuttke, D. S. J. Bioenerg. Biomembr. 1995, 27, 295.

22. Dempsey, J. L.; Winkler, J. R.; Gray, H. B. J. Am. Chem. Soc. 2010, 132, 1060. 
A P P E N D I X B

ELECTRON TRANSFER REACTIONS OF N,N',3,3'-TETRAMETHYL-4,4'BIPYRIDINIUM 
Introduction

The redox potential of the $\mathrm{MV}^{2+} / \mathrm{MV}^{\bullet+}$ couple is $-0.45 \mathrm{~V}$ vs. $\mathrm{SCE}$ in $\mathrm{CH}_{3} \mathrm{CN}$, high enough to drive the reduction of cobaloxime complexes like $\mathrm{Co}\left(\mathrm{dmgBF}_{2}\right)_{2}\left(\mathrm{CH}_{3} \mathrm{CN}\right)_{2}$ (2) to its $\mathrm{Co}^{+}$ counterpart $\left(-0.28 \mathrm{~V}\right.$ vs. SCE) but most certainly not $\mathrm{Co}\left(\mathrm{dmgBF}_{2}\right)_{2}\left(\mathrm{CH}_{3} \mathrm{CN}\right)_{2}(\mathbf{1})(-0.55 \mathrm{~V}$ vs. SCE). However, the derivatization of methyl viologen to N,N'3,3'-tetramethyl-4,4'bipyridinium $\left({ }^{\mathrm{Me}} \mathrm{MV}^{2+}\right.$ ) shifts the one electron potential cathodically by $390 \mathrm{mV}$ (to $-0.84 \mathrm{~V}$ vs. SCE, Figure B.1). The redox potential of this derivative $\left({ }^{\mathrm{Me}} \mathrm{MV}^{2+} /{ }^{\mathrm{Me}} \mathrm{MV}{ }^{\bullet+}\right)$ is significantly more negative than methyl viologen as the radical cation cannot be stabilized via conjugation of the two aryl rings, as the 3,3' methyl substituents prevent the two rings from lying coplanar and stabilizing the added electron via effective $\pi$ - $\pi$ overlap. ${ }^{1-2}$ However, it is important to note that this highly reducing radical cation can no longer be produced by quenching the excited state of $\left[\mathrm{Ru}(\mathrm{bpy})_{3}\right]^{2+}\left(\mathrm{E}^{\mathrm{o}}\left(\mathrm{Ru}^{2+* / 3+}\right)=-0.87 \mathrm{~V}\right.$ vs. SCE $)$. The problem is avoided by utilizing 4,4'-dimethyl-2,2'-bipyridine ( ${ }^{\mathrm{Me}} \mathrm{bpy}$ ) ligands instead of 2,2'-bipyridine to prepare the photosensitizer, $\left[\mathrm{Ru}\left({ }^{\mathrm{Me}} \mathrm{bpy}\right)_{3}\right]^{2+}$, which increases the reductive driving force of $\mathrm{Ru}^{2+*}$ to $-0.99 \mathrm{~V}$ vs. SCE. ${ }^{3-4}$ With an interest of utilizing this alternative electron relay for studies similar to those in Chapter 2, electron transfer studies of $\left[\mathrm{Ru}\left({ }^{\mathrm{Me}} \mathrm{bpy}\right)_{3}\right]^{2+} /{ }^{\mathrm{Me}} \mathrm{MV}^{2+}$ were undertaken.

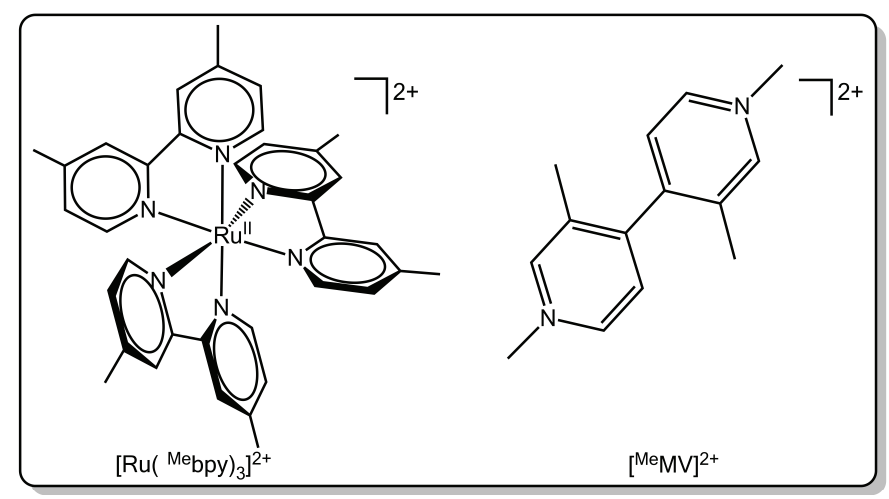

Figure B.1 Photosensitizer and quencher employed in this study. 


\section{Results and Discussion}

This complex is synthetically accessed by the homocoupling of 3-methyl-4-chloro pyridine, ${ }^{5}$ followed by $\mathrm{N}$-methylation and salt exchange to produce the acetonitrile soluble $\mathrm{PF}_{6}$ salt (Figure B.2).

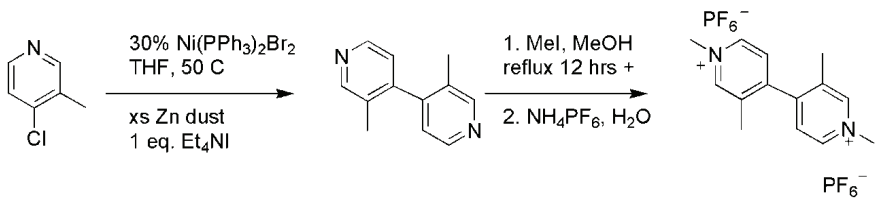

Figure B.2 Synthetic preparation of $\left[{ }^{\mathrm{Me}} \mathrm{MV}\right]\left[\mathrm{PF}_{6}\right]_{2}$

The cyclic voltammogram revealed two reversible one electron reductions, corresponding to ${ }^{\mathrm{Me}} \mathrm{MV}^{2+/{ }^{+}}$and ${ }^{\mathrm{Me}} \mathrm{MV}^{\bullet+/ 0}$ at -0.84 and $-1.03 \mathrm{~V}$ vs. SCE, respectively (Figure B.3). The corresponding reduction potentials for $\mathrm{MV}^{2+}$ occur at $-0.45\left(\mathrm{MV}^{2+/ \bullet+}\right)$ and $-0.87\left(\mathrm{MV}^{\cdot+/ 0}\right) \mathrm{V}$ vs. SCE. As noted above, ${ }^{\mathrm{Me}} \mathrm{MV}^{2+}$ is significantly more difficult to reduce than $\mathrm{MV}^{2+}$, as the 3,3' methyl substituents sterically inhibit conjugation between the two aryl ring, preventing stabilization of the added electron. ${ }^{1,6}$ 


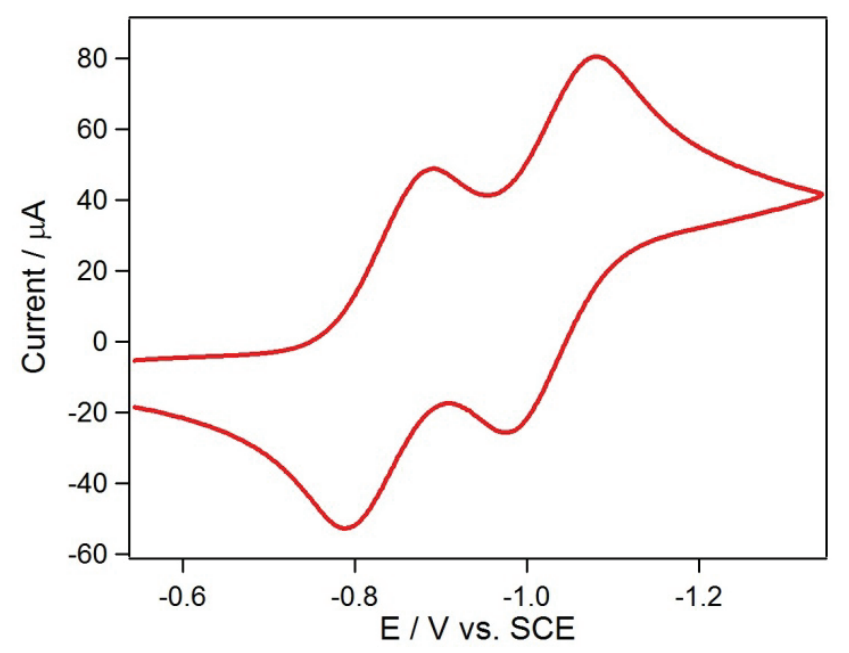

Figure B.3 Cyclic voltammetry of $1.3 \mathrm{mM}\left[{ }^{\mathrm{Me}} \mathrm{MV}\right]\left[\mathrm{PF}_{6}\right]_{2}$ in $0.1 \mathrm{M}$ $\left[{ }^{n} \mathrm{Bu}_{4} \mathrm{~N}\right]\left[\mathrm{ClO}_{4}\right]$.

Spectroelectrochemical measurements were then performed to identify the absorption characteristics of the one-electron reduced viologen, ${ }^{\mathrm{Me}} \mathrm{MV}^{\bullet+}$. Controlled potential electrolysis of ${ }^{\mathrm{Me}} \mathrm{MV}^{2+}$ was carried out at $-0.81 \mathrm{~V}$ vs. SCE and absorption spectra were measured at intervals (Figure B.4). Two absorption features appeared under reducing conditions corresponding to ${ }^{\mathrm{Me}} \mathrm{MV}^{\bullet+}$; a broad structured peak centered at $394 \mathrm{~nm}$ and a broad featureless absorption at $773 \mathrm{~nm} . \mathrm{MV}^{\bullet+}$ has two distinct absorption features, a sharp structured peak centered at $390 \mathrm{~nm}$ and a broad structured peak at $605 \mathrm{~nm}$. The large bathochromic shift and broadening of the low energy absorption feature in ${ }^{\mathrm{Me}} \mathrm{MV}^{\bullet+}$ likely result from the loss of conjugation between the two aryl rings. This low energy absorption feature is due to $\pi^{*}-\pi^{*}$ transitions; without the additional inter-ring conjugation afforded by coplanar rings, this energy gap remains relatively small. ${ }^{6}$ Fine structure is likely lost as there are fewer discrete transitions allowed in the sterically hindered system. 


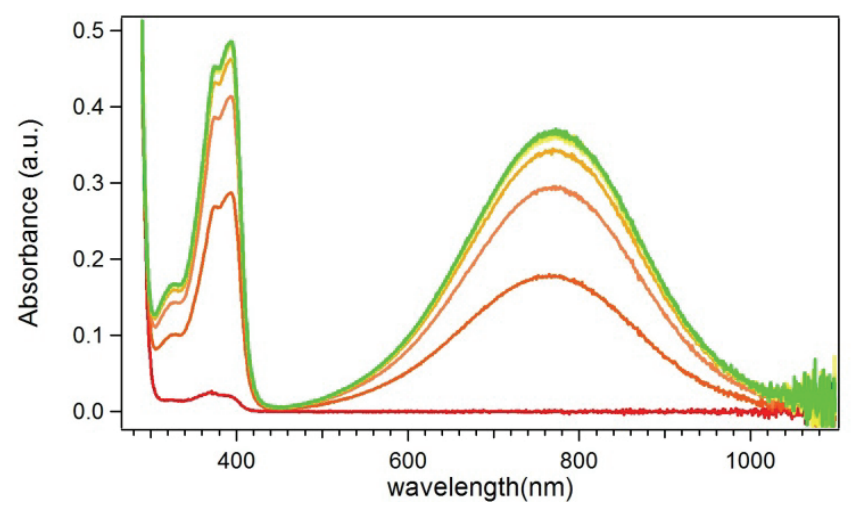

Figure B.4 Spectroelectrochemical measurements of a $2.6 \mathrm{mM}$ ${ }^{\mathrm{Me}} \mathrm{MV}^{2+}$ in $0.1 \mathrm{M}\left[{ }^{n} \mathrm{Bu}_{4} \mathrm{~N}\right]\left[\mathrm{ClO}_{4}\right]$ acetonitrile solution at select time intervals upon controlled electrolysis at $-0.81 \mathrm{~V}$ vs. SCE.

A Stern-Volmer quenching experiment was carried out to determine the rate constant for electron transfer from $\left[\mathrm{Ru}\left({ }^{\mathrm{Me}} \mathrm{bpy}\right)_{3}\right]^{2+^{*}}$ to ${ }^{\mathrm{Me}} \mathrm{MV}^{2+}$. The lifetime of $\left[\mathrm{Ru}\left({ }^{\mathrm{Me}} \mathrm{bpy}\right)_{3}\right]^{2+^{*}}$ in a deoxygenated $0.1 \mathrm{M}\left[{ }^{n} \mathrm{Bu}_{4}\right]\left[\mathrm{PF}_{6}\right]$ acetonitrile solution is $910 \mathrm{~ns}$, slightly shorter than that measured for $\left[\mathrm{Ru}(\mathrm{bpy})_{3}\right]^{2+^{*}}$ under similar conditions $(1 \mu \mathrm{s})$. In aqueous solutions, $\left[\mathrm{Ru}(\mathrm{bpy})_{3}\right]^{2+*}$ has a lifetime of $600 \mathrm{~ns}$, while $\left[\mathrm{Ru}\left({ }^{\mathrm{Me}} \mathrm{bpy}\right)_{3}\right]^{2+^{*}}$ has a notably shorter lifetime of $330 \mathrm{~ns}$, as reported in the literature. ${ }^{7}$ In the presence of ${ }^{\mathrm{Me}} \mathrm{MV}^{2+}$, the excited-state lifetime is attenuated; the Stern-Volmer quenching analysis yielded a quenching rate constant of $5.6 \mathrm{x}$ $10^{7} \mathrm{M}^{-1} \mathrm{~s}^{-1}$. This rate constant is almost two orders of magnitude smaller than that for electron transfer between $\left[\mathrm{Ru}(\mathrm{bpy})_{3}\right]^{2+^{*}}$ and $\mathrm{MV}^{2+}\left(k=1.6 \times 10^{9} \mathrm{M}^{-1} \mathrm{~s}^{-1}\right)$ under identical conditions. The stark difference in electron transfer rate constants can be explained by the difference in driving force between the two electron transfer reactions; electron transfer from $\left[\mathrm{Ru}(\mathrm{bpy})_{3}\right]^{2+*}$ to $\mathrm{MV}^{2+}$ has a $420 \mathrm{mV}$ driving force, while that for electron transfer from $\left[\mathrm{Ru}\left({ }^{\mathrm{Me}} \mathrm{bpy}\right)_{3}\right]^{2+*}$ to ${ }^{\mathrm{Me}} \mathrm{MV}^{2+}$ is only $150 \mathrm{mV}$. 


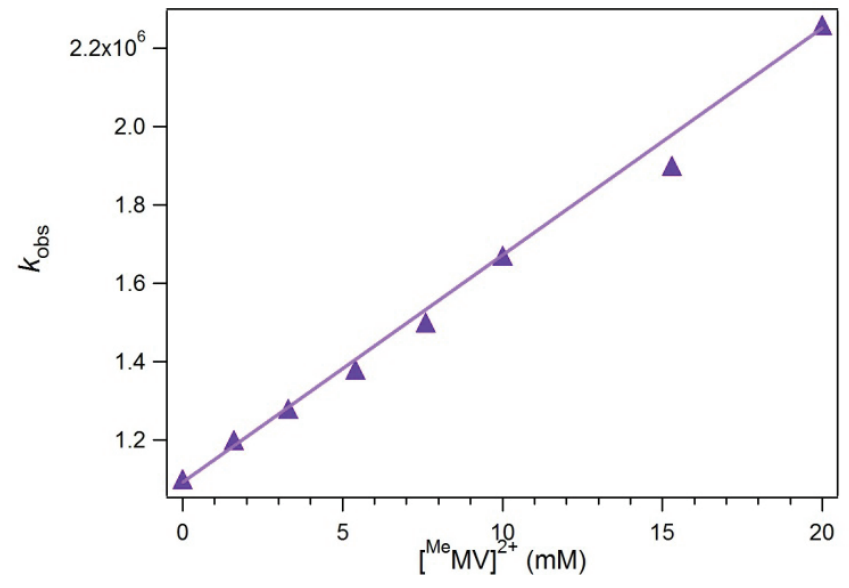

Figure B.5 Stern-Volmer quenching plot for the quenching of $\left[\mathrm{Ru}\left({ }^{\mathrm{Me}} \text { bpy }\right)_{3}\right]^{2+^{*}}$ by ${ }^{\mathrm{Me}} \mathrm{MV}^{2+}$ in $0.1 \mathrm{M}\left[{ }^{n} \mathrm{Bu}_{4}\right]\left[\mathrm{PF}_{6}\right]$ acetonitrile solution. A linear fit to the observed rate constant vs. the concentration of ${ }^{\mathrm{Me}} \mathrm{MV}^{2+}, k_{\mathrm{obs}}=k_{0}+k \mathrm{q}\left[{ }^{\mathrm{Me}} \mathrm{MV}^{2+}\right]$ gives $k_{0}=1.1 \mathrm{x}$ $10^{6} \mathrm{~s}^{-1}$ and $k_{\mathrm{q}}=5.6 \times 10^{7} \mathrm{M}^{-1} \mathrm{~s}^{-1}$.

\section{Conclusions}

Planarization of aryl rings in $\mathrm{MV}^{\cdot+}$ increases $\pi$-conjugation of the system, stabilizing the added electron. Methyl substituents in the 3,3' position of N-methylated bipyridine sterically inhibit this planarization, preventing stabilization of the radical. The electronic properties are consequently altered; the reduction potential is shifted $390 \mathrm{mV}$ negative and the $\pi^{\star}-\pi^{\star}$ transitions in the absorption spectrum are significantly lower in energy. This substituted viologen radical cation is an ideal electron relay with a significantly reducing potential; yet its viability is limited by the availability of ruthenium polypyridine photosensitizers possessing highly reducing photoinduced excited states capable of producing a measurable yield of ${ }^{\mathrm{Me}} \mathrm{MV}{ }^{*+}$ upon electron transfer. ${ }^{7}$ As such, this viologen was not a suitable relay for the proposed studies. 
Experimental Details

Reagents

$\mathrm{CD}_{3} \mathrm{CN}$ was obtained from Cambridge Isotope Laboratories, Inc. All materials, unless noted, were used as received. Tetrabutylammonium hexafluorophosphate and tetrabutylammonium perchlorate were recrystallized from ethanol.

$\left[\mathbf{R u}\left({ }^{\mathrm{Me}} \mathbf{b p y}\right)_{3}\right]\left[\mathbf{P F}_{6}\right]_{2} \quad$ A $100 \mathrm{~mL}$ round bottom was charged with $1.47 \mathrm{~g}$ (8 mmol) 4,4'dimethyl-2,2'bipyridine, $0.5 \mathrm{~g}(2.4 \mathrm{mmol}) \mathrm{RuCl}_{3} \bullet \mathrm{xH}_{2} \mathrm{O}$ and $30 \mathrm{~mL} \mathrm{EtOH}$ and refluxed under atmosphere for $72 \mathrm{hr}$. Solvent was removed via rotorary evaporation to yield a red solid. The solid was rinsed with benzene $(4 \times 50 \mathrm{~mL})$ and $\left[\mathrm{Ru}\left({ }^{\mathrm{Me}} \mathrm{bpy}\right)_{3}\right] \mathrm{Cl}_{2}$ was collected on a frit $(1.56 \mathrm{~g}, 90 \%){ }^{3}$ Salt exchange was performed with $\left[\mathrm{NH}_{4}\right]\left[\mathrm{PF}_{6}\right]$, and crystalline material was obtained via vapor diffusion of diethyl ether into an acetonitrile of $\left[\mathrm{Ru}\left({ }^{\mathrm{Me}} \mathrm{bpy}\right)_{3}\right]\left[\mathrm{PF}_{6}\right]_{2}$. ${ }^{1} \mathrm{H}$ NMR $\left(300 \mathrm{MHz}, \mathrm{CD}_{3} \mathrm{CN}\right): \delta 8.32(\mathrm{~s}, 6 \mathrm{H}), \delta 7.51(\mathrm{~d}, 6 \mathrm{H}), \delta 7.2(\mathrm{~d}, 6 \mathrm{H}), \delta 2.51(\mathrm{~s}, 18 \mathrm{H})$ ppm. UV-Vis $\left(\varepsilon_{458}=14,900\right){ }^{8}$

$\mathbf{N}, \mathbf{N}^{\prime}$-dimethyl-4,4'-bipyridine Synthetic preparation is based on literature techniques for the homocoupling of halopyridines. ${ }^{5}$ A Schlenk flask was charged with Zn powder (1.45 g, $2.21 \mathrm{mmol}), \mathrm{NiBr}_{2}\left(\mathrm{PPh}_{3}\right)_{2}(3.28 \mathrm{~g}, 4.4 \mathrm{mmol})$, and $\left[\mathrm{NEt}_{4}\right] \mathrm{I}(3.79 \mathrm{~g}, 14.7 \mathrm{mmol})$ and placed under an argon atmosphere. $25 \mathrm{~mL}$ of dry THF was added and the reaction mixture was left to stir for $20 \mathrm{~min}$. Next, an $8 \mathrm{~mL}$ THF solution of 4-chloro-3-methyl pyridine (Matrix Scientific) (1.88 g, $14.7 \mathrm{mmol}$ ) was cannula transferred into the reaction mixture over 2 minutes. The reddish reaction was stirred at $50{ }^{\circ} \mathrm{C}$ for $30 \mathrm{hr}$. The cooled solution was quenched with $120 \mathrm{~mL}$ aq. $\mathrm{NH}_{4} \mathrm{OH}(30 \%) .60 \mathrm{~mL}$ of diethyl ether and $60 \mathrm{~mL}$ of benzene were added, and the reaction mixture was filtered over a pad of celite to yield a black precipitate and a yellow solution. The organic layer was separated from the aqueous layer, 
and the aqueous layer was extracted twice with $100 \mathrm{~mL} \mathrm{1:1} \mathrm{diethyl} \mathrm{ether:benzene.} \mathrm{The}$ organic layer was washed with $30 \mathrm{~mL} \mathrm{H} \mathrm{H}_{2} \mathrm{O}$ and then with $30 \mathrm{~mL}$ saturated $\mathrm{NaCl}$ solution, then dried with anhydrous $\mathrm{MgSO}_{4}$. Solvent was removed from the organic layers to yield $1.66 \mathrm{~g}$ of white solid. The residue was chromatographed on silica gel using ethyl acetate as eluent to give 3,3'-dimethy-4,4'-bipyridine (1.62 mmol, 22\%). ${ }^{1} \mathrm{H}$ NMR (300 $\mathrm{MHz}$, $\left.\mathrm{CD}_{3} \mathrm{CN}\right): \delta 8.53(\mathrm{~s}, 2 \mathrm{H}), \delta 8.45(\mathrm{~d}, 2 \mathrm{H}), \delta 7.06(\mathrm{~d}, 2 \mathrm{H}), \delta 2.03(\mathrm{~s}, 6 \mathrm{H}) \mathrm{ppm}$.

\section{N,N',3,3'-tetramethyl-4,4'-bipyridinium bis(hexafluorophosphate) ([ $\left.\left.{ }^{\mathrm{Me}} \mathrm{MV}\right]\left[\mathrm{PF}_{6}\right]_{2}\right)$}

Under an argon atmosphere, a Schlenk flask was charged with 3,3'-dimethy-4,4'-bipyridine $(0.177 \mathrm{~g}, 0.96 \mathrm{mmol})$ in $5 \mathrm{~mL} \mathrm{MeOH}$. Iodomethane $(6 \mathrm{~mL}, 96 \mathrm{mmol})$ was added, and the reaction vessel was wrapped in foil to shield from light. The reaction was refluxed in an oil bath at $45^{\circ} \mathrm{C}$ for $24 \mathrm{hr}$. The solvent and excess iodomethane were removed with vacuum, and the yellow solid, N,N',3,3'-tetramethyl-4,4'-bipyridinium diiodide was dried (0.349 g, 78\%). N,N',3,3'-tetramethyl-4,4'-bipyridinium diiodide (0.349 g, $0.75 \mathrm{mmol})$ was dissolved in $7 \mathrm{~mL} \mathrm{H}_{2} \mathrm{O}$ and brought to $\sim 0{ }^{\circ} \mathrm{C}$ in an ice bath under atmospheric conditions. To this vial, a solution of $\left[\mathrm{NH}_{4}\right]\left[\mathrm{PF}_{6}\right](0.608 \mathrm{~g}, 3.75 \mathrm{mmol})$ in $\sim 2 \mathrm{~mL} \mathrm{H} \mathrm{H}_{2} \mathrm{O}$ was added. A white solid precipitated out immediately, and was collected on a glass frit and rinsed with cold $\mathrm{H}_{2} \mathrm{O}$ and dried under vacuum (0.392 g, 99\%). ${ }^{1} \mathrm{H}$ NMR $\left(300 \mathrm{MHz}, \mathrm{CD}_{3} \mathrm{CN}\right): \delta 8.73$ (s, $\left.2 \mathrm{H}\right), \delta$ $8.65(\mathrm{~d}, 2 \mathrm{H}), \delta 7.8(\mathrm{~d}, 2 \mathrm{H}), \delta 4.35(\mathrm{~s}, 6 \mathrm{H}), \delta 2.2(\mathrm{~s}, 6 \mathrm{H}) \mathrm{ppm} .{ }^{19} \mathrm{~F}$ NMR $\left(282 \mathrm{MHz}, \mathrm{CD}_{3} \mathrm{CN}\right)$ : $\delta=-72(\mathrm{~d}) \mathrm{ppm}$.

\section{Physical Methods}

NMR spectra were recorded using a Varian Mercury 300 spectrometer. ${ }^{1} \mathrm{H}$ NMR chemical shifts were referenced to residual solvents as determined relative to $\mathrm{Me}_{4} \mathrm{Si}(\delta=0 \mathrm{ppm}) .{ }^{19} \mathrm{~F}$ NMR chemical shifts were referenced using an external $\mathrm{C}_{6} \mathrm{~F}_{6}$ standard. UV-visible 
absorption measurements were carried out using a Hewlett Packard 8452 or a Cary 50 $\mathrm{UV}$-Vis spectrophotometer in $1 \mathrm{~cm}$ pathlength quartz cuvettes.

All electrochemical measurements were performed under anaerobic conditions. Cyclic voltammograms were obtained using a $\mathrm{CH}$ Instruments 660 electrochemical analyzer and the data were processed using IGOR Pro. Electrochemical analysis was carried out in a three-electrode cell, consisting of a glassy carbon working electrode (surface area $=0.07$ $\mathrm{cm}^{2}$ ), a platinum wire counter electrode, and a $\mathrm{Ag} / \mathrm{AgNO}_{3}(0.01 \mathrm{M})$ reference electrode filled with $0.1 \mathrm{M}\left[{ }^{n} \mathrm{Bu}_{4}\right]\left[\mathrm{PF}_{6}\right]$ acetonitrile solution. All potentials are referenced to $\mathrm{Fc} / \mathrm{Fc}^{+}$as an internal standard and converted to $\mathrm{SCE}\left(\mathrm{E}^{\circ}\left(\mathrm{Fc} / \mathrm{Fc}^{+}\right)=0.380 \mathrm{~V}\right.$ vs. $\left.\mathrm{SCE}\right)$.

For time-resolved measurements, laser excitation was provided by $8 \mathrm{~ns}$ pulses from a $10 \mathrm{~Hz}$ Q-switched Nd:YAG laser (Spectra-Physics Quanta-Ray PRO-Series). The third harmonic was used to pump an optical parametric oscillator (OPO, Spectra-Physics Quanta-Ray MOPO-700, tunable in the visible region). Probe light for transient absorption kinetics measurements was provided by a $75 \mathrm{~W}$ arc lamp (PTI Model A 1010) that could be operated in continuous wave or pulsed modes. Timing between the laser and the probe light was controlled by a digital delay generator. After passing through the sample collinearly with the laser beam, scattered excitation light was rejected by suitable long pass and short pass filters, and probe wavelengths were selected for detection by a double monochromator (Instruments SA DH-10) with $1 \mathrm{~mm}$ slits. Transmitted light was detected with a photomultiplier tube (PMT, Hamamatsu R928). The PMT current was amplified and recorded with a GageScope transient digitizer. The data were converted to units of $\Delta \mathrm{OD}\left(\Delta \mathrm{OD}=-\log _{10}\left(\mathrm{I} / \mathrm{I}_{0}\right)\right.$, where $\mathrm{I}$ is the time-resolved probe-light intensity with laser excitation, and $\mathrm{I}_{0}$ is the intensity without excitation). Samples were stirred continuously and 
measured using a laser repetition rate of $10 \mathrm{~Hz}$. Data were averaged over approximately 500 shots.

All instruments and electronics in these systems were controlled by software written in LabVIEW (National Instruments). Data were log-time compressed prior to fitting. Data manipulation was performed with MATLAB R2008a (Mathworks, Inc.) and graphed with Igor Pro 5.01 (Wavemetrics).

\section{References}

1. Chen, P.; Curry, M.; Meyer, T. J. Inorg. Chem. 1989, 28, 2271.

2. Willner, I.; Ayalon, A.; Rabinovitz, M. New J. Chem. 1990, 14, 685.

3. Fujita, I.; Kobayashi, H. J. Chem. Phys. 1973, 59, 2902.

4. Kawanishi, Y.; Kitamura, N.; Tazuke, S. Inorg. Chem. 1989, 28, 2968.

5. Iyoda, M.; Otsuka, H.; Sato, K.; Nisato, N.; Oda, M. Bull. Chem. Soc. Jpn. 1990, 63, 80.

6. Creutz, C. Comments Inorg. Chem. 1982, 1, 293.

7. Sutin, N.; Creutz, C. In Inorganic and Organometallic Photochemistry; ACS: 1978; Vol. $168, \mathrm{p} 1$.

8. Creutz, C. Inorg. Chem. 1978, 17, 1046. 
A P P E N D I X C

MATLAB PROGRAMS

Annotated MATLAB programs used for data analysis in Chapters 2 and 3 
을. $\mathrm{m}$ file allows raw data to be processed into TA data (units of OD)

swritten by Jillian Dempsey October 2008

[file, dirpath]=uigetfile('*.lvm', 'select a file');

SOA=input ('input slow amp offset in $\mathrm{V}$ (default 0)');

ollows user to insert slow amp offset

if $S O A>0$;

else;

$\mathrm{SOA}=0 ;$

end;

SOA

scalls a dialog box that will allow one to select a file

data=dlmread(file); sreads numerical data from ascii delimited file "file"

len=length (file); smeasure length of file name

filel=file(1:len-4); osremoves.lvm from file

file2=double(file1); sfilename converted to ascii

file3=[file2, 116, 97, 46, 116, 120, 116]; \%filename with ta.txt appended

file4=char(file3); ofconvert ascii *ta.txt to characters

last=length(file2); oggets $\mathrm{a}, \mathrm{b}$, or $\mathrm{c}$ for timescale $0.1 \mathrm{~ms}=\mathrm{a}, 1 \mathrm{~ms}=\mathrm{b}, 10 \mathrm{~ms}=\mathrm{c}$

filets=file2(last);

tt=data $(:, 2)$; soread the time data out to var tt

yy=data $(:, 3)$; sread the time data out to var yy

$\mathrm{Y}=\mathrm{YY}+\mathrm{SOA} ; \quad$ sadds offset to all $\mathrm{Y}$ data

$\mathrm{k}=25$; ofstarts at data point 25

index $1=0 ; \quad$ ofinds the place where the derivative spikes up to

scorrect for real zero

if filets<99; \& ends with a or b; corresponding to fast amp data deriv=1e5;

else scorresponding to slow amp data deriv=3e2;

end

deriv

while index $1==0$

$\mathrm{k}=\mathrm{k}+1$;

$\operatorname{diff}=(y(k)-y(k-11)) \cdot /(t t(k)-t t(k-11))$;

$\operatorname{diffsq}=(\operatorname{diff})^{\wedge} 2$

$\operatorname{diff} 1$ =sqrt (diffsq) ;

if $\operatorname{diff1}>\operatorname{deriy}$

index $1=\mathrm{k}$

end

end

$\operatorname{diff1}$

t=tt-tt (index 1$)$;

pretrig=index1-10; smakes pretrig end 10 points before diff spike

pretrigy=y(1:pretrig); omakes a string that is the pretrigger data

Io=mean(pretrigy); sofind $\mathrm{y}$ avg of pretrig

index 1

Io

$\mathrm{dOD}=-\log 10(\mathrm{y} . / \mathrm{IO}) ;$ sconvert to $\mathrm{OD}$

plot $(t$, doD) syay graph 


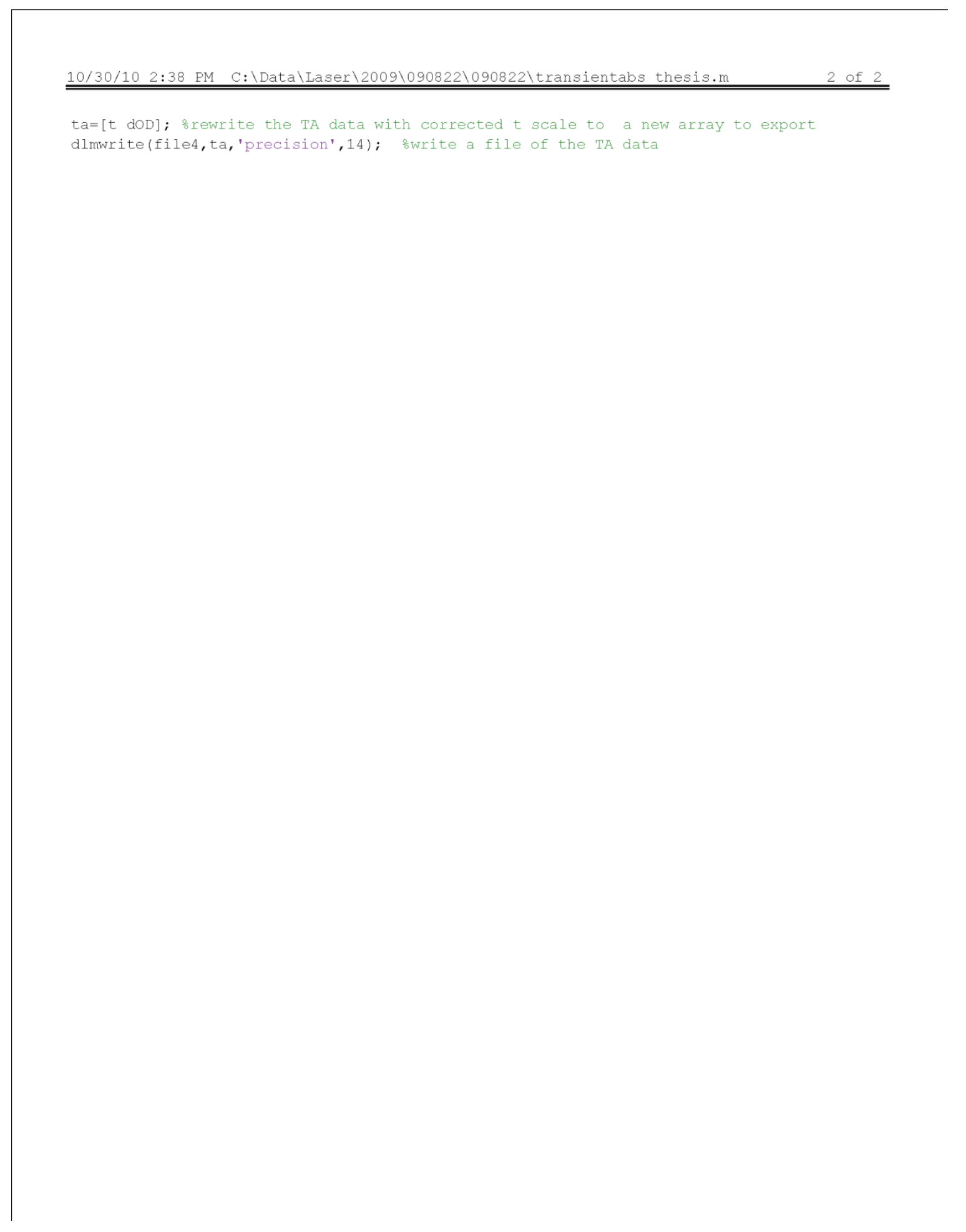


응 INPUTS

oyoff $=y$ axis offset of data, measured at $t=i n f i n i t i$

$8 \mathrm{~s}$ = vector describing the $\mathrm{ET}$ rate constants for the 8 ET reactions

응 ex. $\mathrm{k}=[1.04 \mathrm{e} 6 ; 1.58 \mathrm{e} 9 ; 5.7 \mathrm{e} 9 ; 2 \mathrm{e} ;$; 9.8e9; 5.2e7; 6e6; 4.4e8];

음 $\mathrm{y} 0=$ vector describing initial concentrations;

음 $[[\mathrm{Ru} 2+*] ;[\mathrm{MV} 2+] ;[\mathrm{Ru} 3+] ;[\mathrm{MV}+] ;[\mathrm{Co} 2+] ;[\mathrm{Co}+] ;[\mathrm{CO} 3+]]$

을 ex. y0 $=[5.3 e-6 ; 0.0035 ; 0 ; 0 ; 94 e-6 ; 0 ; 0]$;

8 timespan $=$ timespan utilized in the simulation

8 ex. logtimespan=-6:0.1:-1; timespan=10.^logtimespan;

$[$ Tcalc, Ycalc] $=0$ de23s (e (t, y) jillian8 $(t, y, k)$, timespan, y0) ;

oDifferential equation solver used to compute a simulated concentration

oprofile based on the differential equations describing the

selectron transfer rxns (see jillian8.m)

$\operatorname{eps} 730=[0,0,267,1932,0,7506,0]$;

sepsilon values at $730 \mathrm{~nm}$ for [Ru2+*, [=MV2+, Ru3+, MV+,Co2+,Co+, Co3+]

dODCo_calc23_730=Ycalc*diag $(\operatorname{eps} 730)$;

oconverts Calculated concentration profile into a simulated TA spectrum by

omultiplying the concentration profile by the vector of epsilon values

\%Here the real TA data for the 3 timescales measured are plotted, overlayed

응 one timescale needs to be scaled, include the scaled file here

\%Here the file Co94 is used as an example, manually put into this m-file

semilogx (Co94_730ata $(:, 1)$, Co94_730ata (:,2),'g-');

hold on

semilogx (Co94_730bta $(:, 1)$, Co94_730bta $(:, 2)$, ' $^{-1}$ );

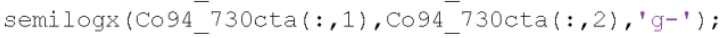

Simulation is overlayed on top of the real data, and correctd with the

syoffset

semilogx (Tcalc, (sum(doDCo_calc23_730')+ yoff),'ko');

$\operatorname{axis}([1 e-61-1 e-3,17 e-3])$;

hold off

8 (sum(dODCo calc23 730') + yoff) should then be saved as a txt file, i.e.

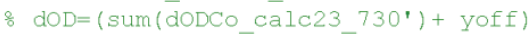

을 save Co94 simulation.txt dOD -ascii -tabs

\& save timecalc.txt Tcalc -ascii -tabs 
function [yprime] =jillian8 $(t, y, k)$

s This function describes the differential equations describing

용 the electron transfer reactions of $\mathrm{Ru}(\mathrm{bpy}) 3^{\wedge} 2+*, \mathrm{MV}^{\wedge} 2+$, and $\mathrm{Co}(\mathrm{dpgBF} 2) 2 \mathrm{~L} 2$

$\%$ as described in Chapter 2. ET reactions and the corresponding

웅 Rate constants k1-k8 are described there

을 $2+*$

yprime $(1)=-\left(k(1) \cdot{ }^{*} y(1)\right)-\left(k(2) \cdot{ }^{*} y(1) \cdot{ }^{*} y(2)\right)$;

$8 \mathrm{MV} 2+$

yprime $(2)=-\left(\mathrm{k}(2) \cdot{ }^{\star} \mathrm{y}(1) \cdot{ }^{\star} \mathrm{y}(2)\right)+\left(\mathrm{k}(3) \cdot{ }^{\star} \mathrm{y}(3) \cdot{ }^{\star} \mathrm{y}(4)\right)+\left(\mathrm{k}(4) \cdot{ }^{\star} \mathrm{y}(4) \cdot{ }^{\star} \mathrm{y}(5)\right)+\left(\mathrm{k}(7) \cdot{ }^{*} \mathrm{y}(7) \cdot{ }^{\star} \mathrm{y}(4)\right) \cdot$

우우

yprime $(3)=\left(\mathrm{k}(2) \cdot{ }^{*} \mathrm{y}(1) \cdot{ }^{*} \mathrm{y}(2)\right)-\left(\mathrm{k}(3) \cdot{ }^{*} \mathrm{y}(3) \cdot{ }^{*} \mathrm{y}(4)\right)-\left(\mathrm{k}(5) \cdot{ }^{*} \mathrm{y}(3) \cdot{ }^{*} \mathrm{y}(6)\right)-\left(\mathrm{k}(6) \cdot{ }^{*} \mathrm{y}(3) \cdot{ }^{*} \mathrm{y}(5)\right) \cdot$

onV+

yprime $(4)=\left(k(2) \cdot{ }^{*} y(1) \cdot{ }^{*} y(2)\right)-\left(k(3) \cdot{ }^{*} y(3) \cdot{ }^{*} y(4)\right)-\left(k(4) \cdot{ }^{*} y(4) \cdot{ }^{*} y(5)\right)-\left(k(7) \cdot{ }^{*} y(7) \cdot{ }^{*} y(4)\right)$

$\circ \mathrm{CO} 2+$

yprime $(5)=\left(\mathrm{k}(5) \cdot{ }^{*} \mathrm{y}(3) \cdot{ }^{*} \mathrm{y}(6)\right)-\left(\mathrm{k}(4) \cdot{ }^{*} \mathrm{y}(4) \cdot{ }^{*} \mathrm{y}(5)\right)-\left(\mathrm{k}(6) \cdot{ }^{*} \mathrm{y}(3) \cdot{ }^{*} \mathrm{y}(5)\right)+\left(\mathrm{k}(7) \cdot{ }^{*} \mathrm{y}(7) \cdot{ }^{*} \mathrm{y}(4)\right)+2{ }^{* \boldsymbol{K}}$

$\left(\mathrm{k}(8) \cdot{ }^{*} \mathrm{Y}(7) \cdot{ }^{*} \mathrm{Y}(6)\right) ;$

$\frac{\mathrm{CO}+}{\mathrm{O}}$

yprime $(6)=\left(k(4) \cdot{ }^{*} \mathrm{y}(4) \cdot{ }^{*} \mathrm{y}(5)\right)-\left(\mathrm{k}(5) \cdot{ }^{*} \mathrm{y}(3) \cdot{ }^{*} \mathrm{y}(6)\right)-\left(\mathrm{k}(8) \cdot{ }^{*} \mathrm{y}(7) \cdot{ }^{*} \mathrm{y}(6)\right)$

응 $3+$

yprime $(7)=\left(k(6) \cdot{ }^{*} \mathrm{y}(3) \cdot{ }^{*} \mathrm{y}(5)\right)-\left(\mathrm{k}(7) \cdot{ }^{*} \mathrm{y}(7) \cdot{ }^{*} \mathrm{y}(4)\right)-\left(\mathrm{k}(8) \cdot{ }^{*} \mathrm{y}(7) \cdot{ }^{\star} \mathrm{y}(6)\right)$;

yprime=yprime(:) 
OVERLAYER

\& Adapted from Overlayer program written by Maraia Ener

splots traces from two different time scales, and asks

ofor multiplyers to adjust the traces

os so that they overlay better

otscale=input('For first round combination, enter 1, \n for second round enter 2 ->');

\% READ DATAFILES

disp('select the fastest trace');

[file, dirpath]=uigetfile('*.txt'); sopens a window to pick my file

if isequal (file,0) || isequal(dirpath,0)

disp ('cancelled!')

hold

$i=0$;

end

filet=[dirpath file];

dataf $=$ dlmread (filet);

disp('select the corresponding longest trace-->');

[file, dirpath]=uigetfile('*.txt'); oopens a window to pick my file

if isequal (file, 0 ) || isequal(dirpath, 0 )

disp ('cancelled!')

hold

$i=0$;

end

return

filet=[dirpath file];

dataL=dlmread (filet);

응 EXTRACT DATA $X$ and $Y$ DATA

$\mathrm{xf}=\operatorname{dataf}(:, 1)$;

yf=dataf $(:, 2)$;

$\mathrm{xL}=\operatorname{dataL}(:, 1)$;

$\mathrm{yL}=\operatorname{dataL}(:, 2)$;

웅 EIRST OVERLAY OF DATA

semilogx ( $\left.\mathrm{xf}, \mathrm{yf}^{\prime} \mathrm{\prime r}^{\prime}\right)$;

hold

semilogx $(x L, y L, ' b ')$;

hold

mult $s=1$;

mult $=1$; 
seguir=input ('does it need adjustment? (y/n) ->', 's');

if seguir $==$ ' $n$ '

$$
\mathrm{yLadj}=\mathrm{yL} \text {; }
$$

end

while seguir $==$ ' $\mathrm{Y}$ ';

multf=input('select a new first trace multiplier (SELECT 1 HERE)->');

if multf $<=0$

multf=1;

disp('defaulting multf=1');

end

multL=input ('select a new second trace multiplier (default 1)->');

if $\operatorname{multL}<=0$

mult $\mathrm{L}=1$;

disp ('defaulting multL=1');

end

yfadj=multf.${ }^{*} y f ;$

yLadj=multL. * $\mathrm{yL}$;

semilogx (xf, yfadj, 'r');

hold

semilogx (xL, yLadj, 'b');

hold

seguir=input('does it need adjustment? $(y / n)$ ', 's');

end

$\operatorname{disp}(\operatorname{multL})$;

datac=combine_jld_thesis ( $x f, y f a d j, x L, y L a d j)$;

$\mathrm{T}=\operatorname{datac}(:, 1) ;$

$Y=\operatorname{datac}(:, 2)$;

$\mathrm{TY}=[\mathrm{T}, \mathrm{Y}]$;

keep=input ('do you want to keep this combined trace? $(y / n)->$ ', 's') ;

if $\mathrm{keep}==$ ' $\mathrm{y}$ ';

[Savefile, Savedirpath, Savefilter]=uiputfile([dirpath,'*.txt'], 'Save overlayed $\boldsymbol{k}$ traces', [dirpath, file]);

eval (['save ' '', Savedirpath, Savefile, '"' TY -ASCII -DOUBLE']);

end 
function datac=combine_jld_thesis ( $x f, y f a d j, x L, y L a d j)$

\%TO CREATE A SINGLE DATA SET COMBINING DATA FOR TWO TIMESCALES

응 Adapted from the combine program written by Maraia Ener

음 INPUTS

o vectors containing time (x) and delta OD (y) data

o key:f \& L are fastest timescale and longer timescale.

o $\mathrm{yL}$ is adjusted (hence yfadj, yLadj) by a multiplier to

\% remove dicontinuities between the data from the three time ranges

OUTPUTS

o datac is a matrix with the combined time values in the first

o column, and delta OD values in the second column

\%Timescales for different purposes

응 tscale $==1$

응 combining the 40 us or 400 us and $40 \mathrm{~ms}$ timescale data

timefl $=1 e-7$

timefu $=3 e-5$

timeLl=3e-5;

\%ADJUSTING FASTER TIMESCALE DATA

scycles to find indexes of the lower and upper bound times

$x l i=1 ;$

while $x f(x l i)<=$ timefl

$x l i=x l i+1$;

end

sui=xli;

while xf(xui) <=timefu

xui=xui+1;

end

newx $1=x f(1: x u i)$;

newy $1=y f a d j(1: x u i)$;

new $1=$ new $11^{\prime}$;

newy $1=$ newy $1^{\prime}$;

웅 ADJUSTING SLOWER TIMESCALE DATA

scycles to find indexes of the lower bound times

xli=1;

while $x L(x l i)<=$ timeLl

$x l i=x l i+1$

end 


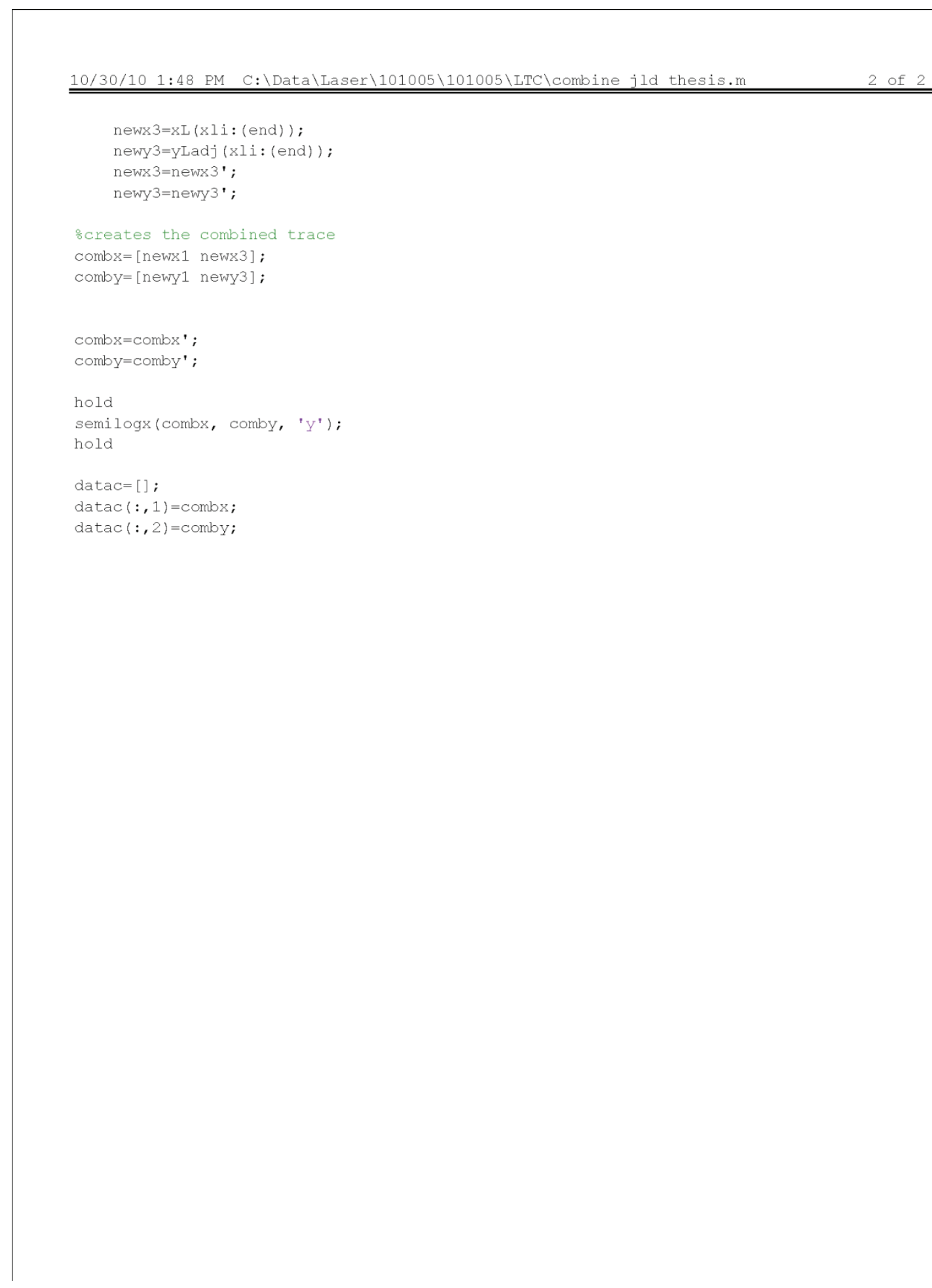

